\title{
Analytic singularities near radial points
}

\author{
Dissertation \\ zur Erlangung des mathematisch-naturwissenschaftlichen Doktorgrades \\ „Doctor rerum naturalium“ \\ der Georg-August-Universität Göttingen \\ im Promotionsprogramm PhD School of Mathematical Sciences (SMS) \\ der Georg-August University School of Science (GAUSS)
}

vorgelegt von

Zheng, Jiguang (郑雯光)

aus HunAN, CHINA

Göttingen, 2014 
Betreuungsausschuss

Prof. Dr. Ingo Witt (Mathematisches Institut)

Prof. Dr. Thomas Schick (Mathematisches Institut)

Mitglieder der Prüfungskommission

Referent: Prof. Dr. Ingo Witt (Mathematisches Institut)

Korreferent: Prof. Dr. Thomas Schick (Mathematisches Institut)

Weitere Mitglieder der Prüfungskommission:

Prof. Dr. Folkert Müller-Hoissen (MPI für Dyn. und Selbstorganisation)

Prof. Dr. Gert Lube (Institut für Num. und Angew. Mathematik)

Prof. Dr. Max Wardetzky (Institut für Num. und Angew. Mathematik)

Prof. Dr. Dorothea Bahns (Mathematisches Institut)

Tag der mündlichen Prüfung: 12 December 2014 


\section{Abstract}

In this thesis, we applied tools of algebraic analysis and knowledge of symplectic geometry and contact geometry to give a normal form of certain class of microdifferential operators, and then studied analytic singularities of solutions with the advantage of normal form. The microdifferential operators are of real analytic coefficients, real principal symbols and simple characteristics near radial points. We linearized the contact vector fields with real analytic coefficients, classified the radial points and find an exact normal form of our operators.

In the last two chapters, by restricting our discussion in the space of Fourier hyperfunctions, first we fully studied the analytic singularities in two dimensional case, and gave some estimates of singularities in higher dimensional cases. Roughly, near an attracting (resp. repelling) generic radial point, we can found solutions with minimal analytic singularity, i.e. the radial direction. Furthermore, near a non-attracting (resp. non-repelling) radial point, if the radial direction is contained in analytic wavefront set of the solution, then either the intersection of analytic wavefront set with stable manifold or with unstable manifold is non-empty. Moreover we discussed solution with prescribed singularities and gave a description of propagation of analytic singularities, especially in three dimensional case. 



\title{
Acknowledgments
}

\author{
Simplex Sigillum Veri.
}

Extra Gottingam non est vita, si est vita, non est ita.

First and foremost, I am using this opportunity to express my deepest gratitude and special thanks to my advisor Professor Ingo Witt for his guidance, assistance and encouragement through the five years. Around him we have a passionate and active group working on diverse subjects related to analysis on partial differential equations. Additionally I also express my sincere thanks to my colleagues and my former colleagues in such group: Dr. Nguyen Nhu Thang, Dr. Nguyen Thi Thu Huong, Dr. Maia Svanadze, Wu Xiaowen, He Daoyin, Ding Bingbing, Nguyen Ngoc Thai, Martin Lippl, Matthias Krüger and Liu Yinbo.

I am particularly in debited to Prof. Chen Hua and Prof. Liu Xiaochun come from Wuhan University. With their help, I went to Göttingen to start my research career. Furthermore, I thank the China Scholarship Council for their financial support of my study at Göttingen.

I especially appreciate all other members of the Research Training Group 1493 "Mathematical Structures in Modern Quantum Physics". We have had lots of great research events like Bonn-Hilbert seminar, Runge-Herglotz seminar, graduate colloquium, mathematical society and so on. It is necessary to record my thankfulness to the following persons in RTG 1493 whom I had helpful discussions with: Prof. Zhu Chenchang, Dr. Li Du, Liu Bei, Suliman Albandik, Dr. Rohit Holkar, Tathagata Banerjee, Dr. Sutanu Roy.

I am grateful to all the friends I have met in Göttingen during those years: Nan Xi, Deng Errui, Luo Cui, Xu Jia, Zhao Yin and others. We have shared lots of beautiful times here. Most importantly, I am very thankful to Tang Yao, my lovely girl, who has been the most significant person in my life.

I wish to thank the following persons for encouragement, suggestions and discussions: Dr. Tang Zuqi, Dr. Liu Yuning, Dr. Yan Youlong, Dr. Zheng Wenjun, Dr. Tu Yinan, Li Songzi, Liu Yang and Dr. Wang Feng.

Finally, my special thanks to my dear parents, my granny, my uncle Tom Liu, other relatives and friends. I feel extremely regretful to my dear parents that I have not spent much time with them during those five years. I hope to make amends in the coming future. 



\section{Contents}

Abstract iii

\begin{tabular}{|lll}
\hline Acknowledgments & v
\end{tabular}

$\begin{array}{lll}1 & \text { Introduction } & 1\end{array}$

1.1 List of notations . . . . . . . . . . . . . . . . . 1

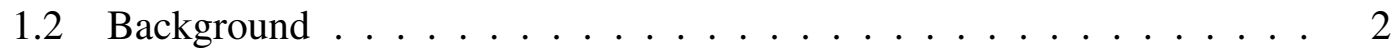

1.3 The problem . . . . . . . . . . . . . . . . 5

1.4 Previous work . . . . . . . . . . . . . . . . . . . . 6

1.5 The main results . . . . . . . . . . . . . . . . . . . . . . . . . . . 14

1.6 Summary . . . . . . . . . . . . . . . . . . 16

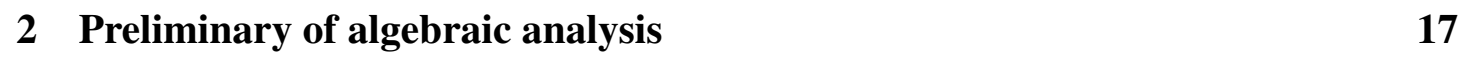

2.1 Sheaf theory and homological algebra . . . . . . . . . . . 17

2.2 Hyperfunction and Microfunctions $\ldots \ldots \ldots$

2.3 Fundamental exact sequences . . . . . . . . . . . . . . . 22

2.4 Fundamental operations . . . . . . . . . . . . . . . . . . . . . 24

2.5 Sheaf $\mathcal{E}_{X}$ of microdifferential operators $\ldots \ldots \ldots 30$

3 Linearization of vector fields 35

3.1 Contact vector fields . . . . . . . . . . . . . . . 35

3.2 Linearization of vector fields . . . . . . . . . . . . . . 37

3.3 Eigenvalues of symplectic mappings . . . . . . . . . . . . . 41

3.4 Birkhoff normal form . . . . . . . . . . . . . . . . . 43

4 The normal form 45

4.1 Subprincipal symbol . . . . . . . . . . . . . . . . . . 45 
4.2 Classification of radial points . . . . . . . . . . . . . . . . 46

4.3 Normal form . . . . . . . . . . . . . . . . . . . . . . 49

4.4 Projected null bicharacteristics $\ldots \ldots \ldots$

5 Analytic singularities in two dimensions 63

5.1 Main results . . . . . . . . . . . . . . . . . . . . 63

5.2 Elliptic case . . . . . . . . . . . . . . 66

5.3 Hyperbolic attracting case . . . . . . . . . . . . . . . 69

5.4 Hyperbolic saddle case . . . . . . . . . . . . . . . . 72

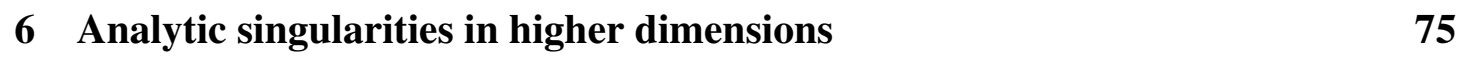

6.1 Attracting case . . . . . . . . . . . . . . . 77

$6.1 .1 \quad$ Elliptic attracting case . . . . . . . . . . . . 77

6.1 .2 Hyperbolic attracting case . . . . . . . . . . . . . 79

6.1 .3 Mixed type attracting case . . . . . . . . . . . . 81

6.2 Non-attracting case . . . . . . . . . . . . . . . . 82

6.2 .1 Hyperbolic non-attracting case . . . . . . . . . . . 83

6.2 .2 Mixed type non-attracting case . . . . . . . . . . . . 87

6.3 Solutions with prescribed singularities $\ldots \ldots . \ldots . \ldots 8$

\begin{tabular}{lr}
\hline Appendices & 95
\end{tabular}

\begin{tabular}{|ll|}
\hline A Symplectic geometry and contact geometry & 97
\end{tabular}

A.1 Symplectic geometry . . . . . . . . . . . . . . . . . . . . 97

A.2 Contact geometry . . . . . . . . . . . . . . . . . . 98

A.3 Quantized contact transformation. . . . . . . . . . . . . . . . 100

A.4 Linearization of real analytic vector field . . . . . . . . . . . . . . 103

\begin{tabular}{|ll}
\hline B Ordinary differential equations & 111
\end{tabular}

B.1 Hypoellipticity of ordinary differential operators . . . . . . . . . . . 111

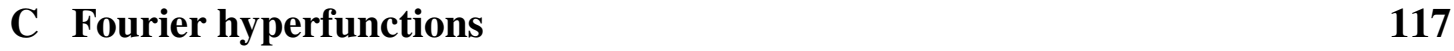

C.1 Fourier transformations . . . . . . . . . . . . . . . . . . . . . 117

C.2 Fourier transformation of hyperfunctions with compact support . . . . . 118

C.3 Fourier transformation of exponentially decreasing hyperfunctions . . . 119

C.4 Fourier transformation of slowly increasing hyperfunctions . . . . . . . 121 
C.5 Embeddings, localization, topology and duality . . . . . . . . . . . 122

\begin{tabular}{|lr|}
\hline D Fourier-Bros-Iagolnitzer transform & 125
\end{tabular}

D.1 Fourier-Bros-Iagolnitzer transform . . . . . . . . . . . . . 125

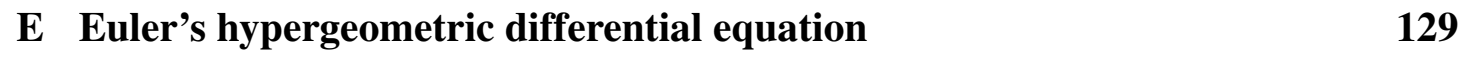

E.1 Euler's hypergeometric differential equation . . . . . . . . . . . . . . . 129

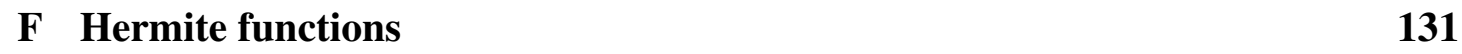

F.1 Hermite functions . . . . . . . . . . . . . . . . . . . . . 131

\begin{tabular}{ll}
\hline References & 133
\end{tabular} 



\section{Chapter 1}

\section{Introduction}

First we give a list of some important notations of the thesis in section 1.1 . Then we provide a short introduction of algebraic analysis in section 1.2 , with a general example to reveal the motivation of using algebraic analysis to study systems of partial differential equations. Later in section 1.3 we state our problem with some assumptions. In section 1.4, we have a short review of previous work related to our problem. The main results of the thesis are listed in section 1.5. At last we give a summary of each chapter and show the relation among chapters.

\subsection{List of notations}

\begin{tabular}{|c|c|c|}
\hline Notation & Description or definition & Reference \\
\hline $\mathcal{A}$ & the sheaf of germs of real analytic functions & Def. 2.2 .3 \\
\hline $\mathcal{A}_{*}$ & the space of rapid decreasing real analytic functions & Def. C.3.1 \\
\hline$\overline{\overline{\mathcal{A}}}$ & the space of slowly increasing real analytic functions & Thm. C.5.2 \\
\hline $\mathscr{B}$ & the sheaf of germs of hyperfunctions & Def. 2.2 .4 \\
\hline $\mathscr{B}_{*}$ & the sheaf of germs of hyperfunctions with compact support & Thm. C.5.1 \\
\hline $\mathscr{B}_{\text {Nils }}$ & the sheaf of hyperfunctions in the Nilsson class & Rmk. B.1.11 \\
\hline $\mathscr{B}[\{0\}]$ & the sheaf of hyperfunctions with support at 0 & Lem. 5.2.2 \\
\hline $\mathscr{B}^{P}$ & the hyperfunction solutions space w. r. t. the operator $P$ & Thm. B.1.6 \\
\hline $\mathscr{C}$ & the sheaf of germs of microfunctions & Def. 2.2.4 \\
\hline $\mathcal{D}$ & the sheaf of rings of differential operators & Section 1.2 \\
\hline $\mathrm{D}$ & the compactification of $\mathbb{R}^{n}: \mathbb{R}^{n} \sqcup S_{\infty}^{n-1}$ & App. C.5 \\
\hline $\mathcal{E}$ & the sheaf of microdifferential operators & Def. 2.5 .7 \\
\hline$\widehat{\mathcal{E}}$ & the sheaf of formal microdifferential operators & Def. 2.5.13 \\
\hline $\mathcal{E}^{\infty}$ & the sheaf of micro-differential operators of infinite order & Def. 2.5.14 \\
\hline $\mathcal{F}$ & Sato's space & Def. C.3.3 \\
\hline $\mathcal{F}^{\prime}$ & Fourier hyperfunctions & App.C.3 \\
\hline $\mathscr{F}(f), \hat{f}$ & the Fourier transformation of $f$ & Def. C.3.8 \\
\hline
\end{tabular}




\begin{tabular}{|c|c|c|}
\hline Notation & Description or definition & Reference \\
\hline$H_{p}$ & the Hamiltonian vector field associated to function $p$ & \\
\hline $\mathscr{M}$ & D-module & Section 1.2 \\
\hline $\mathscr{O}$ & the sheaf of germs of holomorphic functions & Def. 2.2.3 \\
\hline$P_{N}$ & the associated normal form of operator $P$ & \\
\hline $\mathscr{Q}^{-\delta}$ & the sheaf of exponentially decreasing hyperfunctions & Def.C.3.8 \\
\hline $\mathscr{Q}$ & the sheaf of slowly increasing hyperfunctions & Def. $\overline{\text { C.4.1 }}$ \\
\hline $\mathscr{Q}_{*}$ & Sheaf of exponentially decreasing hyperfunctions of all types & App.C.5 \\
\hline$R$ & radial vector field & Def. 4.2 .1 \\
\hline S.S. $(\bullet)$ & singular spectrum & Def. 2.2.7 \\
\hline$T_{h} u$ & the standard FBI transform of $u$ & App. D.1 \\
\hline $\mathrm{WF}_{a}(\bullet)$ & analytic wavefront set & Def.D.1.3 \\
\hline
\end{tabular}

Table 1.1: List of notations

\subsection{Background}

Algebraic analysis, as know as analysis using the algebraic methods of homological algebra and sheaf theory to study analytic (system of) partial differential equations, with the contribution of J. Bernstein, P. Schapira and other French mathematicians, has been extensively developed in the late 1960s by the Kyoto school, M. Sato, T. Kawai, M. Kashiwara and other Japanese mathematicians.

M. Sato published two papers on hyperfunction theory in 1959/60 [64], gave us a new vision to study linear partial differential equations. By using sheaf theory and complex analysis, he constructed the sheaf of germs of hyperfunctions. Then came in 1971 the Master thesis of M. Kashiwara [36], in which he settled the foundations of analytic $\mathcal{D}$-module theory and gave plenty of basic results. It helped to deal with general systems of linear partial differential equations. Two years later, in 1973, Sato created the algebraic microlocal analysis to analyse objects of a manifold in the cotangent bundle, with Kashiwara and Kawai, in their long and delicate paper [63], quoted as [SKK] nowadays everywhere. The phrase "microlocal analysis" refers to the approach to the study of partial differential equations which moves the problem of singularities to the characteristic variety that stays on the cotangent bundle. It is a well developed subject and the classical approach for smooth manifolds can be found in the fundamental work of Hörmander [29], while another approach started from [SKK], so called "algebraic microlocal analysis" 11, deals with the problem in a more algebraic way.

During those years the subject has grown steadily, numerous great results and important theories are acquired in widespread fields of contemporary mathematics, like microlocal analysis, singularities theory, $\mathcal{D}$-module and its applications to representation theory, mathematical physics and so on. For instance, Martinez had shown the Edge-of-the-Wedge theorem, found by a theoretical physicist Bogolyubov in 1956, played an important role in the theory of hyperfunctions and microfunctions [57].

\footnotetext{
${ }^{1}$ For detail, please check G. Kato and D. C. Struppa's book [45].
} 
Study the analytic singularities of solutions is one of the most significant problems in analysis of analytic partial differential equations. Kashiwara, Kawai and Kimura followed Komatsu's terminology "singularity spectrum" 2 in [KKK] [40] to describe the analytic singularities of hyperfunction solutions, while Hörmander introduced the notion of analytic wave-front set to analyze the singularity structure of distribution solutions of linear differential equations. Moreover, Bros and Iagolnitzer also introduced a similar notion "essential support" of a distribution motivated from some physical problems, which can be well studied by the Fourier-Bros-Iagolnitzer (referred to as FBI) Transformation. In the category of distributions, J. M. Bony [5] and some other mathematicians, such as Bros and Iagolnitzer, Kataoka, Nishiwada, and Hill showed independently that those three concepts coincide in the middle of 1970s.

To unravel the powerful algebraic tools in studying systems of partial differential equations, we start with a general example, which can be found in [31], [43], [67].

Let $U$ be an open subset of $\mathbb{C}^{n}$ and $z=\left(z_{1}, \cdots, z_{n}\right)$ be local coordinates of $U$. A (complex analytic linear partial) differential operator on $U$ is an operator of the form

$$
P\left(z, \partial_{z}\right)=\sum_{|\alpha| \leq p} a_{\alpha}(z) \partial_{z}^{\alpha}
$$

where $a_{\alpha}(z) \in \mathscr{O}(U), \alpha=\left(\alpha_{1}, \cdots, \alpha_{n}\right)$ with $|\alpha|=\alpha_{1}+\cdots+\alpha_{n}$ and $\partial_{z}^{\alpha}=\partial_{z_{1}}^{\alpha_{1}} \cdots \partial_{z_{n}}^{\alpha_{n}}$. The restriction of $P$ defined on $U$ to an open subset $V$ is the operator

$$
\left.P\left(z, \partial_{z}\right)\right|_{V}=\left.\sum_{|\alpha| \leq p} a_{\alpha}(z)\right|_{V} \partial_{z}^{\alpha}
$$

The above differential operators form a presheaf $\mathcal{D}_{\mathbb{C}^{n}}$ of rings on $\mathbb{C}^{n}$, and which actually

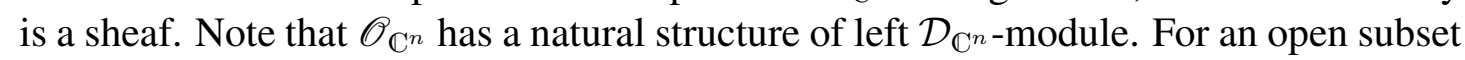
$U$ of $\mathbb{C}^{n}$, denote $\mathcal{D}_{U}$ the restriction to $U$ of the sheaf $\mathcal{D}_{\mathbb{C}^{n}}$.

First consider a differential equation

$$
P u=0
$$

where $P$ is a differential operator defined in $U$ and $u$ is a single unknown function. The set $\operatorname{Hom}_{\mathcal{D}}(\mathscr{M}, \mathscr{O})$ of $\mathcal{D}$-linear homomorphism from $\mathscr{M}$ to $\mathscr{O}$ gives

$$
\begin{aligned}
\operatorname{Hom}_{\mathcal{D}}(\mathscr{M}, \mathscr{O}) & =\operatorname{Hom}_{\mathcal{D}}(\mathcal{D} / \mathcal{D} P, \mathscr{O}) \\
& \simeq\left\{\varphi \in \operatorname{Hom}_{\mathcal{D}}(\mathcal{D}, \mathscr{O}) \mid \varphi(P)=0\right\}
\end{aligned}
$$

where $\mathscr{M}=\mathcal{D} / \mathcal{D} P$ is the left $\mathcal{D}$-module associated with the equation 1.1 . That is

$$
\operatorname{Hom}_{\mathcal{D}}(\mathscr{M}, \mathscr{O}) \simeq\{f \in \mathscr{O} \mid P f=0\}
$$

Hence $\operatorname{Hom}_{\mathcal{D}}(\mathscr{M}, \mathscr{O})$ represents the group of the holomorphic solutions of the equation (1.1).

\footnotetext{
${ }^{2}$ Sato, Kashiwara and Kawai called it "singular spectrum" in [SKK], originally provided by Boutet de Monvel, where "spectrum" first meant the support of the Fourier transform of a function.
} 
Generally, a system of linear partial differential equations of $n$ unknown functions $u_{1}, \cdots, u_{n}$ can be written as

$$
\sum_{j=1}^{n} P_{i j} u_{j}=0, \quad i=1, \cdots, m
$$

A $\mathcal{D}_{U}$-linear morphism $\varphi: \mathcal{D}_{U}^{\oplus m} \rightarrow \mathcal{D}_{U}^{\oplus n}$ with

$$
\varphi\left(Q_{1}, \cdots, Q_{m}\right)=\left(\sum_{i=1}^{m} Q_{i} P_{i 1}, \cdots, \sum_{i=1}^{m} Q_{i} P_{i n}\right)
$$

gives an exact sequence

$$
\mathcal{D}_{U}^{\oplus m} \rightarrow \mathcal{D}_{U}^{\oplus n} \rightarrow \mathscr{M}_{P} \rightarrow 0
$$

where $\mathscr{M}_{p}$ is the cokernel of $\varphi$. Assume $\mathcal{S}$ is the sheaf of solution space, which can be chosen as the space of $C^{\infty}$ functions, the space of Schwartz distributions, the space of holomorphic functions, the space of hyperfunctions and so on. Acting the contravariant functor $\mathscr{H}_{0 m_{\mathcal{D}_{U}}}(\bullet, \mathcal{S})$ then we have the exact sequence

$$
0 \longrightarrow \mathscr{H}^{0} m_{\mathcal{D}_{U}}\left(\mathscr{M}_{P}, \mathcal{S}\right) \longrightarrow \mathcal{S}^{\oplus n} \stackrel{P}{\longrightarrow} \mathcal{S}^{\oplus m}
$$

and hence $\mathscr{H} o m_{\mathcal{D}_{U}}\left(\mathscr{M}_{P}, \mathcal{S}\right) \simeq \mathcal{S}^{P}$ is the solution sheaf. That is, the $\mathcal{D}_{U}$-module $\mathscr{M}_{P}$ represents a homogeneous system of partial differential equations (1.2) naturally.

Assume $u_{1}, \cdots, u_{n} ; v_{1} \cdots, v_{m} \in \Gamma(U, \mathcal{S})$ and

$$
\sum_{j=1}^{n} P_{i j} u_{j}=v_{i}, \quad i=1, \cdots, m .
$$

For any differential operator $Q_{1}, \cdots, Q_{m}$ on $U$, we have

$$
\sum_{j=1}^{n}\left(\sum_{i=1}^{m} Q_{i} P_{i j}\right) u_{j}=\sum_{i=1}^{m} Q_{i} v_{i}
$$

If $\sum_{i=1}^{m} Q_{i} P_{i j}=0$ then $\sum_{i=1}^{m} Q_{i} v_{i}=0$. The algebraic compatibility condition of the system 1.3 is giving by $\left(Q_{1}, \cdots, Q_{m}\right)$. Define $\mathscr{N}_{P}=\operatorname{Ker}(\varphi)$ and $\mathcal{I}_{P}=\operatorname{Im}(\varphi)$, then we have

$$
\begin{aligned}
& 0 \longrightarrow \mathscr{N}_{P} \longrightarrow \mathcal{D}_{U}^{\oplus m} \longrightarrow \mathcal{I}_{P} \longrightarrow 0 \\
& 0 \longrightarrow \mathcal{I}_{P} \longrightarrow \mathcal{M}_{P} \longrightarrow 0
\end{aligned}
$$

where $\varphi=\beta \circ \alpha$. And we get two exact sequences

$$
\begin{gathered}
0 \rightarrow \mathscr{H} \operatorname{om}_{\mathcal{D}_{U}}\left(\mathscr{M}_{P}, \mathcal{S}\right) \rightarrow \mathcal{S}^{\oplus n} \rightarrow \mathscr{H} \operatorname{om}_{\mathcal{D}_{U}}\left(\mathcal{I}_{P}, \mathcal{S}\right) \rightarrow \mathcal{E} x t_{\mathcal{D}_{U}}^{1}\left(\mathcal{M}_{P}, \mathcal{S}\right) \rightarrow 0 \\
0 \rightarrow \mathscr{H} \operatorname{om}_{\mathcal{D}_{U}}\left(\mathcal{I}_{P}, \mathcal{S}\right) \rightarrow \mathcal{S}^{\oplus m} \rightarrow \mathscr{H} o m_{\mathcal{D}_{U}}\left(\mathscr{N}_{P}, \mathcal{S}\right) \rightarrow \mathcal{E} x t_{\mathcal{D}_{U}}^{1}\left(\mathcal{I}_{P}, \mathcal{S}\right) \rightarrow 0
\end{gathered}
$$


The second exact sequence shows

$$
\mathscr{H}_{0 m_{\mathcal{D}_{U}}}\left(\mathcal{I}_{P}, \mathcal{S}\right)=\left\{v \in \mathcal{S}^{\oplus m}: Q v=0, \forall Q \in \mathscr{N}_{P}\right\}
$$

so $\mathcal{I}_{P}$ is a $\mathcal{D}_{U}$-module which represents the system of algebraic compatibility conditions of the system $P$. The first exact sequence shows, the elements of $\mathcal{E} x t_{\mathcal{D}_{U}}^{1}\left(\mathscr{M}_{P}, \mathcal{S}\right)_{x}$ are the class of vectors $v_{x}$ of $\mathcal{S}_{x}^{m}$ satisfying the algebraic compatibility conditions modulo those for which the system is truly compatible.

Moreover, for $k \geq 1$,

$$
\mathcal{E} x t_{\mathcal{D}_{U}}^{k}\left(\mathcal{I}_{P}, \mathcal{S}\right) \simeq \mathcal{E} x t_{\mathcal{D}_{U}}^{k+1}\left(\mathscr{M}_{P}, \mathcal{S}\right)
$$

In summary, the study of the system $(1.3)$ is equivalent to the study of the $\mathcal{D}_{U}$-module $\mathscr{M}_{P}$ and all of its full solution complex $R \mathscr{H}_{o m_{\mathcal{D}_{U}}}\left(\mathscr{M}_{P}, \mathcal{S}\right)$.

We will show a simple example to see the power of $\mathcal{D}$-module. Similar examples can be found in Hotta [30], Kashiwara [35], [SKK] [63]. Assume $\lambda \neq 1$, let us consider two equations

$$
\left(x \frac{d}{d x}-\lambda\right) u=0
$$

and

$$
\left(x \frac{d}{d x}-\lambda+1\right) v=0 .
$$

Even though the two equations look different, they are equivalent to each other by the transformations $u=\frac{1}{\lambda-1} \frac{d}{d x} v$ and $v=x u$. Actually we have the isomorphism

$$
\operatorname{Coker}\left(\mathcal{D} \stackrel{x \frac{d}{d x}-\lambda}{\longrightarrow} \mathcal{D}\right) \simeq \operatorname{Coker}\left(\mathcal{D} \stackrel{x \frac{d}{d x}-\lambda+1}{\longrightarrow} \mathcal{D}\right)
$$

\subsection{The problem}

Let $M$ be an $n$ dimensional real analytic manifold, let $X$ be the complexification of $M$. Let $\mathscr{B}_{M}$ be the sheaf of germs of hyperfunctions with support in $M$, and let $\mathscr{A}_{M}$ be the sheaf of germs of real analytic functions with support in $M$. Let $P$ be a microdifferential operator defined on the cosphere bundle $\sqrt{-1} S^{*} M{ }^{3}$. We want to study the analytic singularities of solution $u \in \mathscr{B}_{M}(M)$ of $P u \in \mathscr{A}_{M}(M)$ for the operator $P$ with real analytic coefficients, real-valued principal symbol $p$, and simple characteristics (i.e. $p=0, d p \neq 0$ on $T^{*} X \backslash 0$. It is a non-degeneracy assumption.) near radial points, where the Hamiltonian vector field $H_{p}$ is nonzero multiple of the radial vector field $R$ in microlocal sense. Here, the non-degeneracy assumption of simple characteristics implies that the radial points of $P$ is microlocally isolated, i.e., there is no other ray near the radial direction consists of radial points.

Even though we introduce the notion of a microdifferential operator and its

\footnotetext{
${ }^{3}$ We follow the notation of $[\mathrm{KKK}][40]$ and $[\mathrm{SKK}][63]$, while the standard coordinates can be choosing as $(x, \sqrt{-1} \xi d x \infty)$.
} 
properties for a complex manifold $X$ and the projective cotangent bundle $P^{*} X$ (or the cosphere bundle $S^{*} X$ ), as in Sato, Kashiwara and Kawai's famous paper [SKK] [63], we would like to follow [KKK] [40] for the notion of microdifferential operators defined on $\sqrt{-1} S^{*} M$ for a real analytic manifold $M$ to deal with our problem. In fact, in $[\mathrm{SKK}]$ the theory is developed to define an operator on a real manifold by restriction, which is more general but less intuitive. Although we have a less general definition of a microdifferential operator then the one in [SKK], it is better to consider our problem with it for the coefficients of our operators are real analytic. We will keep the notion in real case in the following chapters unless a special elaborate.

According to Sato's Fundamental theorem, outside the characteristic variety the operator is invertible, in that case the answer of our problem is trivial, wherefore we will concentrate on the characteristic variety Char $(P)$, which is a closed subset of the cotangent bundle $T^{*} M$. I will use two equivalent notions freely, the singular spectrum S.S.(•) and the analytic wavefront set $\mathrm{WF}_{a}(\bullet)$ to describe the analytic singularities, while the former one stays in $\sqrt{-1} S^{*} M$ and the latter one in $T^{*} M \backslash 0$.

\subsection{Previous work}

Algebraic microlocal analysis concentrates on the real analytic setting, while the classical microlocal analysis usually works in the smooth setting, such as, smooth manifold, smooth coefficients of operators and soon on. By considering the operator near a point that is not radial, there are abundant of results of our problem.

Let $X$ be an smooth (or real analytic) manifold. An operator $P$ is of real principal type if $H_{p} \nVdash R$ on $T^{*} X \backslash 0$, where $R=\sum_{i} \xi_{i} \partial_{\xi_{i}}$ is the radial vector field (also known as Euler vector field) and $p$ is the principal symbol of $P$. Roughly, a point where the condition $H_{p} \nVdash R$ violates is called a radial point $t^{4}$ of $P$, i.e., a radial point is one where the Hamiltonian vector field $H_{p}$ is a multiple of the radial vector field $R$. Due to the assumptions of our problem in section 1.3 , we would like to outline the previous work in Table 1.2 and introduce them as following.

\begin{tabular}{|l|l|l|}
\cline { 2 - 3 } \multicolumn{1}{c|}{} & \multicolumn{1}{c|}{ Smooth setting } & Real-analytic setting \\
\hline Operators of real principal type & L. Hörmander & M. Sato and others \\
\hline Radial point & Guillemin-Schaeffer & To be done \\
\hline
\end{tabular}

Table 1.2: Previous work

\section{(I) Real principal case in the smooth setting.}

Let $X$ be a smooth manifold, let $P \in \Psi^{m}(X)$ be a pseudo-differential operator defined on $X$, and let $p_{m}(x, \xi)$ be the principal symbol of $P$. Assume $p_{m}$ is real-valued and assume $p_{m}=0$ implies $d p_{m} \neq 0$. Such operators are well studied,

\footnotetext{
${ }^{4}$ One can find the exact definition in section 4.2
} 
for instance in Chapter 26 of Hörmander [28], with the assumption that $P$ is of real principal type.

Definition 1.4.1. Let $P$ ba a pseudo-differential operator that satisfies the above conditions, say $P$ is of principal type if $\nabla_{\xi} p_{m} \neq 0$ on the characteristics set

$$
\operatorname{Char}(P)=\left\{(x, \xi) \in T^{*} X \backslash 0 \mid p_{m}(x, \xi)=0\right\}
$$

of $P$.

Remark 1.4.2. A operator $P$ is of principal type if and only if $d p_{m}$ and $\alpha$ are linear independent on $\operatorname{Char}(P)$, where $\alpha=\xi d x$ is the canonical one-form on $T^{*} X \backslash 0$.

Hörmander studied the global solvability of the equation $P u=f$ modulo $C^{\infty}$ and discussed the propagation of singularities.

Theorem 1.4.3. Let $X$ be a smooth manifold and let $P \in \Psi^{m}(X)$ be properly supported and have a real principal symbol $p$. If $u \in \mathcal{D}^{\prime}$ and $P u=f$, then $\mathrm{WF}(u) \backslash \mathrm{WF}(f)$ is contained in $\operatorname{Char}(P)$ and is invariant under the flow defined there by the Hamilton vector field $H_{p}$.

Theorem 1.4.4. Let $P$ be of real principal type in the manifold $X$. Then the following conditions are equivalent:

(a) $P: \mathcal{D}^{\prime} \rightarrow \mathcal{D}^{\prime} / C^{\infty}(X)$ is surjective.

(b) For every compact set $K \subset X$, there is another compact set $K^{\prime} \subset X$ such that

$$
u \in \mathscr{E}^{\prime}(X), \quad \operatorname{sing} \operatorname{supp} P^{*} u \subset K \Longrightarrow \operatorname{sing} \operatorname{supp} u \subset K^{\prime} .
$$

Here $P^{*}$ is the adjoint of $P$.

(c) For every compact set $K \subset X$, there is another compact set $K^{\prime} \subset X$ such that every bicharacteristic interval with respect to $P$ having endpoint over $K$ mush lie entirely over $K^{\prime}$.

\section{(II) Real principal case in the real analytic setting.}

The case that the Hamiltonian vector field $H_{p}$ and Euler vector field $R$ are not collinear was well studied not only in smooth case but also in real analytic setting, i.e. in [KKK] [40], Kato-Struppa [45], [SKK] [63] and so on, they studied the structure of systems of microdifferential equations and obtained several normal forms.

For microdifferential operator of principal type, Sato, Kashiwara and Kawai obtained plenty of profound results in [SKK] [63]. Normal forms are given to analyze the structures of some systems of microdifferential systems. Propagation of analytic singularities have been studied with the advantage of normal forms. 
Theorem 1.4.5. ([KKK] [40], Chap. IV, §3, Theorem 4.3.2) Let $P\left(x, D_{x}\right)$ be a microdifferential operator of order $m$ defined in a neighborhood of $\left(x_{0}, \sqrt{-1} \xi_{0} \infty\right)=(0, \sqrt{-1}(0, \cdots, 0,1) d x \infty)$.

(a) In the case $m=1$, assume the principal symbol $\sigma_{1}(P)=\xi_{1}$, then in a neighborhood of $\left(x_{0}, \sqrt{-1} \xi_{0} \infty\right)$, one has

$$
\mathcal{E} / \mathcal{E} P \cong \mathcal{E} / \mathcal{E} D_{1}
$$

(b) In the case $m \geq 2$, assume the principal symbol $\sigma_{1}(P)=\xi_{1}^{m}$, then in a neighborhood of $\left(x_{0}, \sqrt{-1} \xi_{0} \infty\right)$, one has

$$
\mathcal{E}^{\infty} / \mathcal{E}^{\infty} P \cong \mathcal{E}^{\infty} / \mathcal{E}^{\infty} D_{1}^{m}
$$

Example 1.4.6. Consider two linear differential equations

$$
P\left(x, \partial_{x}\right) u(x)=\partial_{x}^{2} u(x)=0
$$

and

$$
Q\left(y, \partial_{y}\right) v(x)=\partial_{y}^{2} v(y)-\partial_{y} v(y)=0 .
$$

The principal symbols of $P$ and $Q$ are the same, but the structure of solutions are differential. However, there is an intrinsic connection between solutions of each equation, since the above two equations are equivalent as left $\mathcal{E}^{\infty}$-modules, i.e.,

$$
\mathcal{E}^{\infty} / \mathcal{E}^{\infty} P \cong \mathcal{E}^{\infty} / \mathcal{E}^{\infty} Q
$$

In particular, the solution sheaves of hyperfunctions (resp., microfunctions) $\mathscr{B}^{P}$ and $\mathscr{B}^{Q}$ (resp., $\mathscr{C}^{P}$ and $\mathscr{C}^{Q}$ ) are isomorphic.

Theorem 1.4.7. ([KKK] [40], Chap. IV, §3, Theorem 4.3.1) Let $\mathscr{M}$ be an $\mathcal{E}$-module defined in a neighborhood of $\left(x_{0}, \xi_{0}\right) \in T^{*} X \backslash 0$ be such that

(a) There is a left ideal $\mathcal{I}$ such that $\mathscr{M}=\mathscr{M} / \mathcal{I}$.

(b) The zero set $V(J)$ of $J:=\cup_{m}\left\{\sigma_{m}(P) \mid P \in(I) \cap \mathcal{E}(m)\right\}$ is a non-singular manifold of codimension d in a neighborhood of $\left(x_{0}, \xi_{0}\right)$, and the canonical one-form does not vanish on $V(J)$.

(c) The zero set $V(J)$ is real.

(d) The totality of $\xi$-homogeneous analytic functions which vanishes on $V(J)$ is $J$. Via a quantized contact transformation $\mathscr{M}$ can be transformed into

$$
\mathscr{N}: \mathcal{E} /\left(\mathcal{E} D_{1}+\mathcal{E} D_{2}+\cdots+\mathcal{E} D_{n}\right) .
$$

Remark 1.4.8. The system $\mathscr{N}$ is sometimes called a de Rham system, or a partial de Rham system.

Definition 1.4.9. Let $V$ be an involutive submanifold of $S^{*} \mathbb{R}^{n}$ satisfying (b) and (c) in Theorem 1.4.7, and suppose

$$
V=\left\{(x, \xi) \in S^{*} M: f_{1}(x, \xi)=\cdots=f_{d}(x, \xi)=0\right\} .
$$


The bicharacteristic manifold $B=B_{\left(x_{0}, \xi_{0}\right)}$ associated with $\mathrm{V}$ and passing through $\left(x_{0}, \xi_{0}\right) \in V$ is the $d$-dimensional integral manifold through $\left(x_{0}, \xi_{0}\right)$ of the $d$ Hamiltonian operators

$$
H_{j}: \sum_{k=1}^{n}\left(\frac{\partial f_{j}}{\partial \xi_{k}} \frac{\partial}{\partial x_{k}}-\frac{\partial f_{j}}{\partial x_{k}} \frac{\partial f_{j}}{\partial \xi_{k}}\right), \quad j=1, \cdots, d .
$$

Theorem 1.4.10. ( [45], Chap. 6, §6.5, Theorem 6.5.6) Let $\mathscr{M}$ be the $\mathcal{E}$-module as in Theorem 1.4.7. Then in a neighborhood of $\left(x_{0}, \xi_{0}\right)$ the microfunction solution sheaf $\mathscr{H}_{\mathcal{O}} m_{\mathcal{E}}(\mathscr{M}, \mathcal{C})$ is supported in $V$ and is locally constant along each bicharacteristic manifold. Moreover, $\mathscr{H}_{\mathrm{o}}(\mathscr{M}, \mathcal{C})$ is a flabby sheaf in the direction transversal to bicharacteristic manifolds, and $\mathcal{E} x t_{\mathcal{E}}^{j}(\mathscr{M}, \mathcal{C})=0$ for $j \neq 0$.

Remark 1.4.11. Say a sheaf $\mathscr{F}$ is flabby in the direction transversal to bicharacteristic manifolds, it means there is a manifold $U^{\prime}$, a flabby sheaf $\mathscr{F}^{\prime}$ on $U^{\prime}$ and a smooth morphism $\psi: U \cap V \rightarrow U^{\prime}$ such that the bicharacteristic manifolds in $U \cap V$ are the fibers of $\psi$ and $\left.\mathscr{F}\right|_{U \cap V} \cong \psi^{-1} \mathscr{F}^{\prime}$.

From Theorem, we have the following two features. The flabbiness of the sheaf of microfunction solutions is a restatement of the propagation of singularities along bicharacteristic manifolds. The vanishing of the higher Ext functors shows the system $\mathscr{M}$ is locally solvable, by giving suitable algebraic compatibility conditions.

Theorem 1.4.12. ([KKK] [40], Chap. IV, §3, Theorem 4.3.5) Let $P$ be a microdifferential operator of order $m$ defined in a neighborhood of $\left(x_{0}, \xi_{0}\right) \in$ $T^{*} X \backslash 0$ and $p_{m}(x, \xi)$ is the principal symbol. Assume $\left\{p_{m}, \bar{p}_{m}\right\} \supsetneqq 0$, then the equation $P u=0$ can be transformed into the following equation $\mathscr{N}$, defined in a neighborhood of $(y, \sqrt{-1} \eta)=\left(0 ; \sqrt{-1}\left(0^{\prime}, 1\right)\right)$ by an invertible real quantized contact transformation:

$$
\mathscr{N}:\left(\frac{\partial}{\partial y_{1}}-\sqrt{-1} y_{1} \frac{\partial}{\partial y_{n}}\right) u=0
$$

Remark 1.4.13. If we assume $\left\{p_{m}, \bar{p}_{m}\right\} \supsetneqq 0$, then the corresponding normal equation is

$$
\mathscr{N}:\left(\frac{\partial}{\partial y_{1}}+\sqrt{-1} y_{1} \frac{\partial}{\partial y_{n}}\right) u=0
$$

in a neighborhood of $(y, \sqrt{-1} \eta)=\left(0 ; \sqrt{-1}\left(0^{\prime}, 1\right)\right)$.

Remark 1.4.14. The condition $\left\{p_{m}, \bar{p}_{m}\right\} \neq 0$ implies $d p_{m} \nVdash \alpha$ for the canonical one-form $\alpha$, which is coincide with the non-radial condition, or the operator is of real principal type.

Remark 1.4.15. Under the assumption of Theorem 1.4.7. $V=\left\{p_{m}(x, \xi)=0\right\}$ and $\bar{V}=\left\{\bar{p}_{m}=0\right\}$ intersect transversally and $\left.\alpha\right|_{V \cap \bar{V}}$ defines a contact structure on $V \cap \bar{V}$. When $\operatorname{codim}(V \cap \bar{V})=2$ and $V \cap \bar{V}$ has contact structure $\left.\alpha\right|_{V \cap \bar{V}}$, the normal equation corresponding to $P u=0$ is given by

$$
\left(\partial_{y_{1}} \pm \sqrt{-1} y_{1}^{k} \partial_{y_{n}}\right) u=0 .
$$


Theorem 1.4.16. ([KKK] [40], Chap. IV, §3, Theorem 4.3.6) Let $P\left(x, D_{x}\right)=$ $D_{1}-\sqrt{-1} x_{1} D_{n}$, and let $Q\left(x, D_{x}\right)=D_{1}+\sqrt{-1} x_{1} D_{n}$. Then there is a non-zero microlocal operator $\mathscr{K}$ defined in a neighborhood of $\left(x_{0}, \sqrt{-1} \xi_{0} \infty\right)=$ $\left(0 ; \sqrt{-1}\left(0^{\prime}, 1\right) \infty\right) \in \sqrt{-1} S^{*} \mathbb{R}^{n}$ such that the sequence

$$
0 \longrightarrow \mathscr{C} \stackrel{Q}{\longrightarrow} \mathscr{C} \stackrel{\mathscr{K}}{\longrightarrow} \mathscr{C} \stackrel{P}{\longrightarrow} \mathscr{C} \longrightarrow 0
$$

is exact.

Hence, $P$ is solvable in a neighborhood of $\left(x_{0}, \sqrt{-1} \xi_{0} \infty\right)$, while $Q$ is not solvable, and the image of $Q$ is characterized as the kernel of $\mathscr{K}$.

Theorem 1.4.17. ([KKK] [40], Chap. IV, §3, Theorem 4.3.7) Let $P\left(x, D_{x}\right)$ be a microdifferential operator of order $m$, which is defined in a neighborhood of $\left(x_{0} ; \sqrt{-1} \xi_{0} \infty\right) \in \sqrt{-1} S^{*} M$, and let $p_{m}(x, \sqrt{-1} \xi)$ be the principal symbol of $P$. Then one has

(i) If $p_{m}\left(x_{0}, \sqrt{-1} \xi_{0}\right)=0$ and $\left\{p_{m}, \bar{p}_{m}\right\}\left(x_{0}, \sqrt{-1} \xi_{0}\right) \supsetneqq 0$, then $P$ is epimorphic in a neighborhood of $\left(x_{0} ; \sqrt{-1} \xi_{0} \infty\right)$ and $\operatorname{Ker}(P)$ is equal to the image of a microlocal operator $\mathscr{K}$.

(ii) If $p_{m}\left(x_{0}, \sqrt{-1} \xi_{0}\right)=0$ and $\left\{p_{m}, \bar{p}_{m}\right\}\left(x_{0}, \sqrt{-1} \xi_{0}\right) \lesseqgtr 0$, then $P$ is monomorphic in a neighborhood of $\left(x_{0} ; \sqrt{-1} \xi_{0} \infty\right)$ but not epimorphic, and $\operatorname{Im}(P)$ is equal to the kernel of a microlocal operator $\mathscr{K}$. That is, for the equation $\mathrm{Pu}=\mathrm{g}$ to be solvable, $\mathscr{K} g=0$ must hold.

There are systems have characteristic varieties which not satisfy the condition of Theorem 1.4.7, we will introduce two of them in the following statement.

Theorem 1.4.18. ( [45], Chap. 6, §6.5, Theorem 6.5.7) Let $\mathscr{M}=\mathcal{E} / \mathcal{I}$ be a system of microdifferential equations in one unknown with simple characteristics. Assume its characteristic variety $V$ satisfies

(i) $V \cap \bar{V}$ is a non-singular involutive manifold;

(ii) $V \cap \bar{V}$ intersect transversally;

(iii) $\left.\omega\right|_{V \cap \bar{V}} \neq 0$,

where $\bar{V}$ is the complex conjugate of $V$, then the system $\mathscr{M}$ is microlocally equivalent to the partial Cauchy-Riemann system

$$
\mathscr{N}:=\frac{\partial u}{\partial \bar{z}_{j}}:=\frac{1}{2}\left(\frac{\partial}{\partial x_{2 j-1}}+\sqrt{-1} \frac{\partial}{\partial x_{2 j}}\right) u=0, \quad j=1, \cdots, d,
$$

where $d$ is the codimension of $V$.

To state the propagation of regularity results for this systems, first we give an extra definition.

Definition 1.4.19. Let $M$ be a system of finite order microdifferential operators whose codimension $d$ characteristic variety $V$ satisfy all three conditions of Theorem 1.4.18. The $2 d$-dimensional bicharacteristic manifold of $V \cap \bar{V}$ through $\left(x_{0}, \sqrt{-1 \xi_{0} \infty}\right)$ is called virtual bicharacteristic manifold of $M$. 
Remark 1.4.20. The bicharacteristic manifold is invariant under contact transformation, and the virtual bicharacteristic manifold is invariant under real contact transformations.

Remark 1.4.21. The virtual bicharacteristic manifold of the system $\mathscr{N}$ in Theorem 1.4 .18 through $\left(x_{0}, \sqrt{-1} \xi_{0} \infty\right)$ is given by

$$
\left\{(x, \sqrt{-1} \xi \infty): x_{j}=\left(x_{0}\right)_{j} \quad \text { for } \quad j=2 d+1, \cdots, n ; \xi=\xi_{0}\right\} .
$$

Then we have

Theorem 1.4.22. Let $\mathscr{M}=\mathcal{E} / \mathcal{I}$ be as in Theorem 1.4 .18 and let $U$ be any open subset in the virtual bicharacteristic manifold of $\mathscr{M}$. Then every microfunction solution of $\mathscr{M}$ which vanishes in $U$ also vanishes everywhere in the virtual bicharacteristic manifold.

In the year 1957, H. Levy [53] gave an equation

$$
\left(\frac{1}{2}\left(\partial_{x_{1}}+\sqrt{-1} \partial_{x_{2}}\right)-\left(x_{1}+\sqrt{x_{2}}\right) \partial_{x_{3}}\right) u=f
$$

without local solutions, which greatly promoted the solvability problems of linear partial differential equations. The work of $\mathrm{H}$. Lewy on solvability theory induced the study of so called Lewy-Mizohata type systems.

Definition 1.4.23. ([SKK] [63], Chap. III, §2.3) Let $M$ be a real manifold. Let an involutive submanifold $V$ in a complex neighborhood of $\left(x_{0}, \sqrt{-1} \xi_{0} d x \infty \in\right.$ $\sqrt{-1} S^{*} M$ be written as

$$
\left\{(x \cdot \sqrt{-1} \xi d x \infty) \mid p_{1}(x, \sqrt{-1} \xi)=\cdots=p_{d}(x, \sqrt{-1} \xi)=0\right\} .
$$

Then the generalized Levi form $L(x, \xi)$ of $V$ is the Hermitian matrix whose coefficients are the Poisson brackets

$$
\left\{p_{j}, \bar{p}_{k}\right\}_{1 \leq j, k \leq d}
$$

Remark 1.4.24. The signature of the generalized Levi form is independent of the choice of the defining functions $p_{j}$, and is also invariant under a real contact transformation.

Theorem 1.4.25. ([SKK] [63], Chap. III, §2.3) Let $\mathscr{M}=\mathcal{E} / \mathcal{I}$ be an $\mathcal{E}$-module defined in a neighborhood of $\left(x_{0}, \sqrt{-1} \xi_{0} \infty\right)$ and which satisfies the conditions (b) and $(d)$ in Theorem 1.4.7 If the generalized Levi form of $V(J)$ has $p$ positive eigenvalues and $d-p$ negative eigenvalues at $\left(x_{0}, \sqrt{-1} \xi_{0} \infty\right)$, then $\mathscr{M}$ is microlocally equivalent to the $(p, 1-p)$-Lewy-Mizohata system

$$
\mathscr{N}_{p}:= \begin{cases}\left(\partial_{x_{j}}-\sqrt{-1} x_{j} \partial_{x_{n}}\right) u=0 & j=1, \cdots, r \\ \left(\partial_{x_{j}}+\sqrt{-1} x_{j} \partial_{x_{n}}\right) u=0 & j=p+1, \cdots, d .\end{cases}
$$


In the local theory of linear partial differential equations, with the above discussion we have an elegant structure theorem for general systems:

Theorem 1.4.26. ([SKK] [63], Chap. III, §2.4) Let $\mathscr{M}$ be admissible and regular system of micro-differential equations such that $V \cap \bar{V}$ is regular $5 T_{x}(V) \cap$ $T_{x}(\bar{V})=T_{x}(V \cap \bar{V})$ for any $x \in V$, and such that its generalized Levi form is of constant signature $(p, q)$. Then $\mathscr{M}$ is microlocally isomorphic to a direct summand of the direct sum if a finite number of copies of the system $\mathscr{N}$ which, in a suitable neighborhood, has the following form:

$$
\mathscr{N}:= \begin{cases}\partial_{x_{j}} j u=0 & j=1, \cdots, p \\ \left(\partial_{x_{r+2 k-1}}+\sqrt{-1} \partial_{x_{r+2 k}}\right) u=0 & k=1, \cdots, s \\ \left(\partial_{x_{r+2 s+l}}+\sqrt{-1} x_{r+2 s+l} \partial_{x_{n}}\right) u=0 & l=1, \cdots, q \\ \left(\partial_{x_{r+2 s+l}}-\sqrt{-1} x_{r+2 s-l} \partial_{x_{n}}\right) u=0 & l=q+1, \cdots, p+q\end{cases}
$$

where $r=2 \operatorname{codim}(V)-\operatorname{codim}(V \cap \bar{V})$ and $s=\operatorname{codim}(V \cap \bar{V})-\operatorname{codim}(V)-$ $(p+q)$.

\section{(III) Radial case in the smooth setting.}

However, in the radial point case we only have a few results in smooth setting, such as [18], [22], [75]. In Guillemin and Schaeffer's paper [22], they studied the normal form of $P$ in the non-resonant case, and had the result:

Theorem 1.4.27. ([GS] [22]) Let $P: C^{\infty}(X) \rightarrow C^{\infty}(X)$ be a second order pseudo-differential operator with real principal symbol on $n$ dimensional smooth manifold $X$. Let $\nu^{0}=\left(x_{0}, \xi_{0}\right)$ be a generic radial point ${ }^{6}$ of $P$. Then $P$ can be microlocally conjugated to a second order differential operator on $\mathbb{R}^{n}$ of the form

$$
P_{N}=\left\langle A \partial_{x^{\prime}}, \partial_{x^{\prime}}\right\rangle+\left\langle B x^{\prime}, \partial_{x^{\prime}}\right\rangle \partial_{x_{n}}+\left\langle C x^{\prime}, x^{\prime}\right\rangle \partial_{x_{n}}^{2}+\gamma x_{n} \partial_{x_{n}}^{2}+\theta \partial_{x_{n}} .
$$

Here $A, B, C$ are constant $(n-1) \times(n-1)$ matrices, $A$ and $C$ are symmetric, $x=\left(x^{\prime}, x_{n}\right), \gamma \in \mathbb{R} \backslash 0$ and $\theta \in \mathbb{C}$.

With additional conditions on the generic radial points, the normal form $P_{N}$ has simpler forms. More specifically, near a hyperbolic radial point $\left(x_{0}, \xi_{0}\right)$, a pseudo-differential operator $P$ of first order is microlocally equivalent to

$$
P_{N h}=\left\langle\tilde{B} x^{\prime}, \partial_{x^{\prime}}\right\rangle+\gamma x_{n} \partial_{x_{n}}+\theta .
$$

Here $\tilde{B}$ is a constant $(n-1) \times(n-1)$ matrix.

Near an elliptic radial point $\left(x_{0}, \xi_{0}\right)$, a pseudo-differential operator $P$ of seconder order is microlocally equivalent to

$$
P_{N e}= \pm\left(\left\langle\tilde{A} \partial_{x}, \partial_{x}\right\rangle+\left\langle\tilde{C} x^{\prime}, x^{\prime}\right\rangle \partial_{x_{n}}^{2}\right)+\gamma x_{n} \partial_{x_{n}}+\theta \partial_{x_{n}}
$$

\footnotetext{
${ }^{5}$ See Definition A.2.8.

${ }^{6}$ See Definition 4.2.4.
} 
Here $\tilde{A}$ is a $(n-1) \times(n-1)$ identity matrix, $\tilde{C}$ is $a(n-1) \times(n-1)$ diagonal matrix with all non-vanishing entries larger than $\gamma^{2} / 16$.

Remark 1.4.28. Elliptic (resp. hyperbolic) radial point $]^{7}$ are determined by the eigenvalues of a linear symplectic mapping at the generic radial point.

Then they discussed the propagation of singularities by taking the advantage of normal forms. At first they fully studied the propagation of singularities in two dimensional case:

Theorem 1.4.29. Let $P$ be a pseudo-differential operator in Theorem 1.4.27 and let the dimension $n=2, \nu_{0}=(0,0 ; 0,1), \gamma=1$. Set $\mathbb{R}_{+} \nu_{0}=\left\{\left(0 ; 0^{\prime}, \eta\right) \mid \eta>0\right\}$. Then

(i) when the radial point $\nu_{0}$ is elliptic, there is a solution of the equation $P u=$ $f$ with $f \in C^{\infty}(X)$ such that the minimal wave front set is $\mathrm{WF}(u)=\mathbb{R}_{+} \nu_{0}$.

(ii) when the radial point $\nu_{0}$ is hyperbolic attractor/repellor, there is a solution of the equation $P u=f$ with $f \in C^{\infty}(X)$ such that the minimal wave front set is $\mathrm{WF}(u)=\mathbb{R}_{+} \nu_{0}$.

(iii) when the radial point $\nu_{0}$ is hyperbolic saddle, and if $\nu^{0} \in \mathrm{WF}(u)$, then one of the four projected null bicharacteristic curves 8 which go asymptotically to $\nu^{0}$ must intersect $\mathrm{WF}(u)$ :

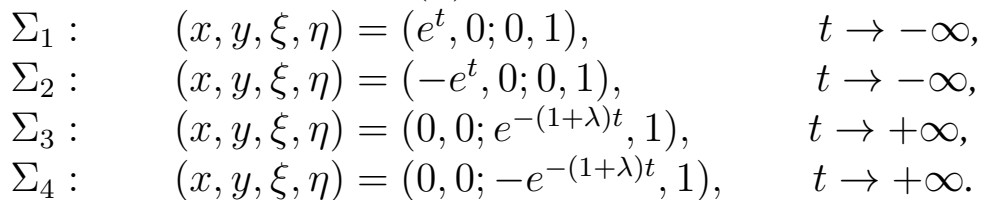

That is, there are 3 minimal wave front sets: $\Sigma_{1} \cup \mathbb{R}_{+} \nu_{0}, \Sigma_{2} \cup \mathbb{R}_{+} \nu_{0}$ and $\Sigma_{3} \cup \Sigma_{4} \cup \mathbb{R}_{+} \nu_{0}$.

In high dimensional situation, they only had results in a special case, as know as attracting (resp. repelling) case.

Theorem 1.4.30. Let $P$ be a pseudo-differential operator in Theorem 1.4.27, and set the dimension of the smooth manifold satisfies $n \geq 3$. Then if the radial point $\nu^{0}$ is attracting/repelling 9 there is a solution of the equation $P u=f$ with $f \in C^{\infty}(X)$ such that $\mathrm{WF}(u)$ consists of the minimal singularity, i.e., the radial direction.

There are also some other results related, such as A. Hassell, R. Melrose and A. Vasy[26] worked on scattering problem near radial point in smooth setting. And J. R. S. Filho studied the propagation of singularities near radial points in smooth setting.

\section{(IV) Radial case in the real analytic setting.}

\footnotetext{
${ }^{7}$ See Definitions 4.2 .5

${ }^{8}$ See section 4.4 for detail.

${ }^{9}$ See Definition 5.1 .1
} 
Even though recently we have more and more research working on the behaviors near radial points, all of them are done in smooth setting. For the radial case in the real analytic setting, before this paper there is no research on it. In my paper, I have extended Guillemin and Schaeffer's results [22] in the smooth setting to real analytic setting, and also discussed the non-attracting (resp. non-repelling) case. The main results are listed in next section.

\subsection{The main results}

We list the main results in three parts.

\section{(A) The normal form.}

Theorem 1.5.1. Let $\mathscr{M}$ and $\mathscr{M}^{\prime}$ are two $\mathcal{E}_{X}$-modules associated with two $m$-th order micro-differential operators $P$ and $P^{\prime}$, respectively, and let $\nu_{0}$ and $\nu_{0}^{\prime}$ be radial points of $P$ and $P^{\prime}$, respectively. If $\mathscr{M}$ near $\nu_{0}$ and $\mathscr{M}^{\prime}$ near $\nu_{0}^{\prime}$ are microlocally equivalent, via quantized contact transformation, then one has three invariants:

(i) $\gamma_{P}^{m-1}\left(\nu_{0}\right)=\gamma_{P^{\prime}}^{m-1}\left(\nu_{0}^{\prime}\right)$,

(ii) $\left.\sigma_{\text {sub }}^{(m-1)}(P)\right|_{\nu_{0}}=\left.\sigma_{\text {sub }}^{(m-1)}\left(P^{\prime}\right)\right|_{\nu_{0}^{\prime}}$,

(iii) There is a linear symplectic map $W: \widehat{E}_{\hat{\nu}_{0}} \rightarrow \widehat{E}_{\hat{\nu}_{0}^{\prime}}$ such that

$$
A_{P^{\prime}}^{m-1}\left(\nu_{0}^{\prime}\right)=W A_{P}^{m-1}\left(\nu_{0}\right) W^{-1}
$$

If $\nu_{0}$ and $\nu_{0}^{\prime}$ are generic, then these conditions are also sufficient, that is, the three invariants imply that $\mathscr{M}$ and $\mathscr{M}^{\prime}$ are microlocally equivalent.

As a corollary, we have

Corollary 1.5.2. Let $P \in \mathcal{E}(2)$ be a second order micro-differential operator on a real analytic manifold $M$. Let $\mathscr{M}$ be the $\mathcal{E}$-module associated with $P$, i.e., $\mathscr{M} \simeq \frac{\mathcal{E}}{\mathcal{E} P}$. Furthermore if $\nu_{0}=\left(x_{0}, \xi_{0}\right)$ is a generic radial point of $P$, then near $\left(x_{0}, \xi_{0}\right)$, via quantized contact transformation, one can transform $\mathscr{M}$ into the system

$$
\mathscr{N} \simeq \frac{\mathcal{E}}{\mathcal{E} P_{N}}
$$

and $P_{N}$ is the normal form of $P$ of form

$$
P_{N}=\left\langle A D_{x^{\prime}}, D_{x^{\prime}}\right\rangle+\left\langle B x^{\prime}, D_{x^{\prime}}\right\rangle D_{x_{n}}+\left\langle C x^{\prime}, x^{\prime}\right\rangle D_{x_{n}}^{2}+\gamma x_{n} D_{x_{n}}^{2}+\theta D_{x_{n}} .
$$

Here $A, B, C$ are constant $(n-1) \times(n-1)$ matrices, $A$ and $C$ are symmetric, $x=\left(x^{\prime}, x_{n}\right), \gamma \in \mathbb{R}$ and $\theta \in \mathbb{C}$. 
More specially, if $\nu_{0}$ is hyperbolic, then near $\nu_{0}, \mathscr{M}$ is microlocally equivalent to the $\mathcal{E}_{\mathbb{R}^{n}}$-module $\mathscr{N} \cong \frac{\mathcal{E}}{\mathcal{E} P_{N h}}$ via quantized contact transformation, where

$$
P_{N h}=\left\langle\tilde{B} x^{\prime}, D_{x^{\prime}}\right\rangle+\gamma x_{n} D_{x_{n}}+\theta .
$$

Here $\tilde{B}$ is a constant $(n-1) \times(n-1)$ matrix.

If $\nu_{0}$ is elliptic, then near $\nu_{0}, \mathscr{M}$ is microlocally equivalent to the $\mathcal{E}_{\mathbb{R}^{n} \text {-module }}$ $\mathscr{N} \cong \frac{\mathcal{E}}{\mathcal{E} P_{N e}}$ via quantized contact transformation, where

$$
P_{N e}=\left\langle\tilde{A} D_{x^{\prime}}, D_{x^{\prime}}\right\rangle+\left\langle\tilde{C} x^{\prime}, x^{\prime}\right\rangle D_{x_{n}}^{2}+\gamma x_{n} D_{x_{n}}^{2}+\theta D_{x_{n}} .
$$

Here $\tilde{A}$ is a $(n-1) \times(n-1)$ identity matrix, $\tilde{C}=\left(\tilde{c}_{i}\right)$ is a $(n-1) \times(n-1)$ diagonal matrix with all non-vanishing entries larger than $\gamma^{2} / 16$.

(B) Analytic singularities in two dimensional case.

Theorem 1.5.3. Let $P$ be a second order micro differential operator defined on a two dimensional real analytic manifold $M$, with real-analytic coefficients, real principal symbol $p$, and simple characteristics, and $\left(x_{0}, \xi_{0}\right)$ is a generic radial point of $P$. Consider the equation

$$
P_{N} u=f
$$

in space of hyperfunctions, where $f$ is a real analytic function.

(1) If $\left(x_{0}, \xi_{0}\right)$ is an elliptic radial point, then there exists a solution $u \in \mathscr{B}$ such that $\mathrm{WF}_{a}(u)=\{(0,0,0, \eta) \mid \eta>0\}$.

(2) If $\left(x_{0}, \xi_{0}\right)$ is hyperbolic attracting/repelling radial point and $0<\lambda<1$, then there exist a solution $u \in \mathscr{B}$ such that $\mathrm{WF}_{a}(u)=\{(0,0,0, \eta) \mid \eta>0\}$.

(3) If $\left(x_{0}, \xi_{0}\right)$ is hyperbolic saddle radial point and $\lambda \notin[0,1]$, and if $\{(0,0,0, \eta) \mid \eta>0\} \subset \mathrm{WF}_{a}(u)$, then at least one of the following projected null bicharacteristics which go asymptotically to $(0,0,0,1)$ as $t \rightarrow \mp \infty$ : $(x, y, \xi, \eta)=\left( \pm e^{t}, 0,0,1\right)$ and $(x, y, \xi, \eta)=\left(0,0, \pm e^{-(1+\lambda) t}, 1\right)$ will be contained in $\mathrm{WF}_{a}(u)$. Furthermore, if one of the later two projected null bicharacteristics is contained in $\mathrm{WF}_{a}(u)$, so is the other.

\section{(C) Analytic singularities in higher dimensional case.}

First, if the radial point is either attracting or repelling, we have:

Theorem 1.5.4. Let $\left(x_{0}, \xi_{0}\right)$ be a generic radial point which is either attracting or repelling. Then there exist a hyperfunction $u$ such that $P_{N} u$ is real analytic and $\mathrm{WF}_{a}(u)$ just consists of the minimal singularities, i.e., the radial direction.

If the radial point is neither attracting nor repelling, we have 
Theorem 1.5.5. Let $u \in \mathscr{B}\left(\mathbb{R}^{n}\right)$ be a hyperfunction solution of $P_{N} u \in \mathcal{A}\left(\mathbb{R}^{n}\right)$ such that the radial point $\left(0 ; 0^{\prime}, 1\right) \in \mathrm{WF}_{a}(u)$, then

$$
\mathrm{WF}_{a}(u) \cap \Gamma_{1} \neq \emptyset \quad \text { or } \quad \mathrm{WF}_{a}(u) \cap \Gamma_{2} \neq \emptyset .
$$

where $\Gamma_{1}$ and $\Gamma_{2}$ are stable and unstable manifolds, respectively.

Moreover, we construct solutions with prescribed singularities for hyperbolic non-attracting case, and we calculate the projected null bicharacteristics and discuss the propagation of analytic singularities, especially in three dimensional case.

\subsection{Summary}

We give a brief account of the content of the thesis.

In Chapter 2 we introduced the basic theory of algebraic analysis, i.e., some rudimentary knowledge in sheaf theory, homological algebra, the elementary knowledge of hyperfunctions and microfunctions and related fundamental operations, the microdifferential operators and so on.

In Chapter 3 we found the way to linearize a contact vector field with real analytic coefficients near a hyperbolic equilibrium point, and analyzed the eigenvalues of symplectic mappings associated with the linear contact vector field. To go though this chapter, one may needs Appendix A for preparation.

Chapter 4 is concerned with the normal form of our operators near a generic radial point. First we give the definition of subprincipal symbol with some properties, then we clarify the classification of radial points and generic condition, thereafter we study the normal form and finally set forth the projected null bicharacteristics.

In Chapter 5 we completely studied the analytic singularities of solutions in two dimensional space. Near an elliptic radial point, our equations can be transformed to the standard Euler's hypergeometric equations, which has been well studied. Near hyperbolic attracting radial points, we discussed problem in the category of Fourier hyperfunctions, which can be found in Appendix $C$.

In Chapter 6 we have studied the analytic singularities of solutions in higher dimensional space. We have discussed the results for attractor/repeling radial points and non-attracting/non-repelling radial points respectively. Furthermore, we discussed solutions with prescribed singularities for hyperbolic radial points and discussed the projected null bicharacteristics in three dimensional case. In additional, for elliptic radial points, the equation can be transformed to a Schrödinger equation of a string with uncoupled oscillators via Fourier transformation and coordinates transformation. Solutions of such equation are Hermite functions introduced in Appendix $\mathrm{F}$ 


\section{Chapter 2}

\section{Preliminary of algebraic analysis}

In this chapter, we have a quick glance of the fundamental knowledge of algebraic analysis. The main references are Kaneko [34], [KKK] [40], Kashiwara-Schapira [45] and [SKK] [63].

\subsection{Sheaf theory and homological algebra}

First we have a short review of sheaf theory and homological algebra.

Definition 2.1.1. A presheaf $F$ over a topological space $X$ associates with each open subset $U$ of $X$ an abelian group $F(U)$, such that there is an abelian group homomorphism, which is known as the restriction map,

$$
\rho_{V, U}: F(U) \rightarrow F(V)
$$

for open subsets $U \supset V$ with the following axioms:

(1) The map $\rho_{U, U}$ is the identity map on $F(U)$,

(2) For three open subsets of $X, U_{1} \subset U_{2} \subset U_{3}$, we have

$$
\rho_{U_{1}, U_{2}} \circ \rho_{U_{2}, U_{3}}=\rho_{U_{1}, U_{3}} .
$$

For $s \in F(U)$, denote $\rho_{V, U}(s)$ as $\left.s\right|_{V}$ for short.

The stalk of the presheaf $F$ at $x \in X$ is defined as

$$
F_{x}=\underset{x \in U}{\lim _{x \in U}} F(U)
$$

where $\underset{\longrightarrow}{\longrightarrow}$ denotes the inductive limit, for $U$ running over all the neighborhood of $x$.

Definition 2.1.2. A presheaf $\mathscr{F}$ over $X$ is said to be sheaf if the following axioms are satisfied: it is given an open covering $\left\{U_{i}\right\}_{i \in I}$ of $U$ in $X, U=\bigcup_{i \in I} U_{i}$ and $I$ is an index set. 
(1) Let $s \in \mathscr{F}(U)$, if $\left.s\right|_{U_{i}}=0$ for each $i \in I$, then $s=0$.

(2) Suppose that for each $i \in I$ there exists $s_{i} \in \mathscr{F}\left(U_{i}\right)$ such that $\left.s_{i}\right|_{U_{i} \cap U_{j}}=\left.s_{j}\right|_{U_{i} \cap U_{j}}$ for $i, j \in I$. Then there exists $s \in \mathscr{F}(U)$, such that $\left.s\right|_{U_{i}}$ for each $i \in I$.

Definition 2.1.3. Let $F$ be a presheaf over $X$. A sheaf $\mathscr{F}$ is said to be the sheaf associated with the presheaf (or $\mathscr{F}$ is the sheafification of $F$, or $\mathscr{F}$ is the induced sheaf from the presheaf $F$ ) if for each open subset $U$ of $X$ the presheaf $\mathscr{F}(U)$ (which is a sheaf actually) associates all the maps

$$
U \stackrel{s}{\rightarrow} \bigcup_{x \in U} F_{x},
$$

satisfying that for each $x \in U$ there is a neighborhood $U^{\prime}$ of $x$ and $s^{\prime} \in F\left(U^{\prime}\right)$ such that $s\left(x^{\prime}\right)=s_{x^{\prime}}^{\prime}$ is true for any $x^{\prime} \in U^{\prime}$.

Definition 2.1.4. Let $X$ and $Y$ be topological spaces and let $f: X \rightarrow Y$ be a continuous map. For a sheaf $\mathscr{F}$ over $X$, the direct image of $\mathscr{F}$ under the continuous map $f$, denoted by $f_{*}(\mathscr{F})$, is a presheaf $U \rightarrow \mathscr{F}\left(f^{-1} U\right)$ over $Y$, which is a sheaf indeed. Moreover, for a sheaf $\mathscr{G}$ on $Y$ there can be define the presheaf $\lim _{V \supset f(U)} \mathscr{G}(V)$ for an open subset $U$ of $X$. This presheaf is not a sheaf in general, and the associated sheaf is called the inverse image of $\mathscr{G}$ under $f$, denoted by $f^{-1}(\mathscr{G})$.

Definition 2.1.5. Let $\mathscr{F}$ be a sheaf over a topological space $X$, and let $U$ be an open subset of $X$. Denote the support of $s$ by

$$
\operatorname{supp} s:=\left\{x \in U \mid s_{x} \neq 0 \text { for } s \in \mathscr{F}(U)\right\} .
$$

Definition 2.1.6. Let $\mathscr{F}$ be a sheaf over a topological space $X$ and let $S$ be a locally closed subset of $X$. Then define

$$
\Gamma_{S}(X, \mathscr{F}):=\{s \in \mathscr{F}(U) \mid \operatorname{supp} s \subset S\}
$$

where $U$ is open in $X$ and $S$ is closed in $U$.

In the case $S=X$, we denote $\Gamma_{S}(X, \mathscr{F})$ with $\Gamma(X, \mathscr{F})$. In general we also denote that $\mathscr{F}(U)$ with $\Gamma(U, \mathscr{F})$ for an open set $U$ in $X$.

Definition 2.1.7. A sheaf $\mathscr{F}$ over a topological space $X$ is said to be flabby if for an arbitrary open subset $U$, the homomorphism

$$
\rho_{U, X}: \mathscr{F}(X) \rightarrow \mathscr{F}(U)
$$

is an epimorphism. Therefore, for a flabby sheaf $\mathscr{F}$, any section of $\mathscr{F}$ over $U$ can be extended to a section over $X$.

Definition 2.1.8. An exact sequence

$$
0 \rightarrow \mathscr{F} \rightarrow \mathscr{L}^{0} \rightarrow \mathscr{L}^{1} \rightarrow \cdots
$$

is said to be a flabby resolution of a sheaf $\mathscr{F}$ if each $\mathscr{L}^{j}, j=0,1, \cdots$, is a flabby sheaf. We can find a canonical flabby resolution for arbitrary sheaf. 
Definition 2.1.9. Let $X$ and $Y$ be topological spaces, let $f: X \rightarrow Y$ be a continuous map, and let $\mathscr{F}$ be a sheaf over $X$. Then denote

$$
\Gamma_{f-\operatorname{proper}}(X, \mathscr{F})=\left\{s \in \Gamma(X, \mathscr{F})|f|_{\text {supp } s} \text { is a proper map }\right\} .
$$

Given an open subset $U$ of $Y$, let $f_{!}(\mathscr{F})$ be the sheaf over $Y$ associated with the presheaf $\Gamma_{f-\text { proper }}\left(f^{-1}(U), \mathscr{F}\right)$. Furthermore, the sheaf $R^{k} f_{!}(\mathscr{F})$ over $Y$ denotes the sheaf associated with the presheaf $H^{k}\left(\Gamma_{f-\operatorname{proper}}\left(f^{-1}(U), \mathscr{L}^{\bullet}\right)\right)$, where $0 \rightarrow \mathscr{F} \rightarrow \mathscr{L} \bullet$ is a flabby resolution of $\mathscr{F}$.

Definition 2.1.10. Let $\mathscr{A}$ be a sheaf of rings on a topological space $X$.

(1) An $\mathscr{A}$-module $\mathscr{M}$ is called of finite type (resp. of finite presentation) if for any point $x \in X$ there is a neighborhood $U$ and an exact sequence

$$
\left.\left.0 \leftarrow \mathscr{M}\right|_{U} \leftarrow \mathscr{A}^{m}\right|_{U}
$$

for some $n \in \mathbb{N}$ (resp. $\left.\left.\left.0 \leftarrow \mathscr{M}\right|_{U} \leftarrow \mathscr{A}^{n}\right|_{U} \leftarrow \mathscr{A}^{m}\right|_{U}$ for some $n, m \in \mathbb{N}$ ).

(2) An $\mathscr{A}$-module $\mathscr{M}$ is called pseudo-coherent, if any submodule of finite type defined on an open subset is of finite presentation. If $\mathscr{M}$ is pseudo-coherent and of finite type, then $\mathscr{M}$ is called coherent.

(3) An $\mathscr{A}$-module $\mathscr{M}$ is called Noetherian if $\mathscr{M}$ satisfies the following properties:

- $\mathscr{M}$ is coherent.

- For any $x \in X, \mathscr{M}_{x}$ is a Noetherian $\mathscr{A}_{x}$-module, that is, any increasing sequence of $\mathscr{A}_{x}$ )-submodules is stationary.

- For any open subset $U$, any increasing sequence of coherent $\left(\left.\mathscr{A}\right|_{U}\right)$-submodules of $\left.\mathscr{M}\right|_{U}$ is locally stationary.

\subsection{Hyperfunction and Microfunctions}

Mikio Sato introduced the theory of hyperfunctions in [64] via two aspects of motivation. On one hand, he believe that the real analytic setting is more natural than the smooth setting as in Schwartz's theory of distributions when we consider the theory of differential equations. On the other hand, his background in theoretical physics arouse him to analyze the boundary values of holomorphic functions, which leads to hyperfunctions. It is much more intuitive to understand hyperfunction as sum of boundary values of holomorphic functions, rather than the cohomological definition of the sheaf of hyperfunctions. One can check Kaneko [34], Kato-Struppa [45] and Morimoto [59] for details. We will give both the algebraic definition and the intuitive definition.

Definition 2.2.1. Let $M$ be an $n$-dimensional real analytic manifold. If $X$ is a complex manifold of dimension $n$ containing $M$ such that locally $M \cong \mathbb{R}^{n} \hookrightarrow \mathbb{C}^{n} \cong X$, then $X$ is said to be a complexification of $M$. That is, there is a neighborhood $\Omega$ of each point $x \in X$ and an injective map $f: \Omega \rightarrow \mathbb{C}^{n}$ such that $\Omega \cap M=f^{-1}\left(\mathbb{R}^{n}\right)$. 
Definition 2.2.2. Let $\mathbb{Z}_{X}$ be the constant sheaf on $X$ such that each stalk is $\mathbb{Z}$, with $\mathbb{Z}$ be the ring of rational integers. The orientation sheaf $\omega_{M}$ over an $n$-dimensional real analytic manifold $M$ is defined as $\mathscr{H}_{M}^{n}\left(\mathbb{Z}_{X}\right)$.

Definition 2.2.3. Let $M$ be a real analytic manifold and $X$ be a complexification of $M$. We denote the sheaf of germs of real analytic functions on $M$ by $\mathcal{A}(M)$. That is, for an open subset $V$ of $M$, it is the sheaf associated with the presheaf $\mathcal{A}(V)=\{$ real analytic functions defined on $V\}$. Moreover, we denote the sheaf of germs of holomorphic functions on $X$ by $\mathscr{O}_{X}$. That is, for an open subset $U$ of $X$, it is the sheaf associated with the presheaf $\mathscr{O}(U)=\{$ holomorphic functions defined on $U\}$

Definition 2.2.4. We have the following definitions:

$$
\begin{gathered}
\mathscr{B}_{M}=\mathscr{H}_{M}^{n}\left(\mathscr{O}_{X}\right) \otimes_{\mathbb{Z}} \omega_{M} \\
\mathscr{C}_{M}=\mathscr{H}_{\sqrt{-1} S^{*} M}^{n}\left(\pi^{-1} \mathscr{O}_{X}\right)^{a} \otimes \pi^{-1} \omega_{M},
\end{gathered}
$$

where $\pi: \sqrt{-1} S^{*} M \rightarrow M$ is canonical projection and $a: \sqrt{-1} S^{*} M \rightarrow \sqrt{-1} S^{*} M$ maps $(x, \xi)$ to $(x,-\xi)$ is antipodal map. Especially we have $\mathscr{B}_{M}=\left.\mathscr{C}_{M}\right|_{M}$. And one calls $\mathscr{C}_{M}$ (resp. $\mathscr{B}_{M}$ ) the sheaf of microfunctions (resp., hyperfunctions) on $M$. The sections of $\mathscr{C}_{M}$ (resp., $\mathscr{B}_{M}$ ) are called microfunctions (resp., hyperfunctions).

Both of the sheaf $\mathscr{B}_{M}$ of hyperfunctions and the sheaf $\mathscr{C}_{M}$ of microfunctions are flabby sheaves. Proof of this can be seen from Kaneko [34], [KKK] [40].

In contrast to the abstract definition, there is a intuitive way to define hyperfunctions, and the latter is much useful in practical use, see Kaneko [34], Morimoto [59].

Definition 2.2.5. Let $F_{j}(z)$ be a holomorphic function defined on an infinitesimal wedge ${ }^{1} \Omega+\sqrt{-1} \Gamma_{j} 0(j=1, \cdots, N)$. The following commutative formal sum, as know as boundary-value representation

$$
f(x)=\sum_{j=1}^{N} F_{j}\left(x+\sqrt{-1} \Gamma_{j} 0\right)
$$

is called a hyperfunction on $\Omega$. The functions $\left\{F_{j}(z)\right\}_{j=1}^{N}$ is called a set of defining functions of $f(x)$. If $\Gamma_{i} \cap \Gamma_{j} \neq 0$, then

$$
F_{j}\left(x+\sqrt{-1} \Gamma_{j} 0\right)+F_{k}\left(x+\sqrt{-1} \Gamma_{j} 0\right)=\left(F_{j}+F_{k}\right)\left(x+\sqrt{-1} \Gamma_{j} \cap \Gamma_{k} 0\right)
$$

If $F(z)$ is holomorphic on the infinitesimal wedge $\Omega+\sqrt{-1} \Gamma_{j} 0$, then the local Bochner theorem 2 grantees that $F(z)$ is holomorphic on $\Omega+\sqrt{-1} \widehat{\Gamma}_{j} 0$. Here $\widehat{\Gamma}_{j}$ is the convex hull of $\Gamma_{j}$. Hence, we can assume each $\Gamma_{j}$ in the above definition is convex.

One can show the above two definitions of hyperfunctions are equivalent via Čech cohomology theory, see [34], [40], [59] and [63].

\footnotetext{
${ }^{1}$ Roughly, an infinitesimal wedge $\Omega+\sqrt{-1} \Gamma 0 \in \sqrt{-1} S M$ is an open set which approaches a wedge of the opening $\Gamma$ asymptotically in the vicinity of the edge $\Omega$. For accurate definition, see Kaneko [34], Definition 2.2.9.

${ }^{2}$ See [34], Page 82.
} 
Here are some examples of hyperfunctions and microfunctions.

Example 2.2.6. The space $\mathscr{B}(\Omega)$ of hyperfunction on an open subset $\Omega \subset \mathbb{R}$ can be defined as

$$
\mathscr{B}(\Omega):=\underset{U \supset \Omega}{\lim } \mathscr{O}(U \backslash \Omega) / \mathscr{O}(U) .
$$

We give some hyperfunctions of one variable.

(a) (Heaviside function) $Y(x)=\left[\frac{1}{2 \pi \sqrt{-1}} \log (-z)\right]_{z=x}$, the holomorphic function in brackets is the defining function. For a holomorphic function $F(z),[F(z)]_{z=x}=$ $F(x+\sqrt{-1} 0)-F(x-\sqrt{-1} 0)$.

(b) (Dirac's delta function) $\delta(x)=\left[-\frac{1}{2 \pi \sqrt{-1} z}\right]_{z=x}$. Moreover, the Dirac's delta function of several variables is of the form

$$
\begin{aligned}
\delta\left(x_{1}, \cdots, x_{n}\right) & =\delta\left(x_{1}\right) \cdots \delta\left(x_{n}\right)=\prod_{i=1}^{n}\left[-\frac{1}{2 \pi \sqrt{-1} z_{i}}\right]_{z_{i}=x_{i}} \\
& =\frac{1}{(-2 \pi \sqrt{-1})^{n}} \sum_{\sigma} \frac{\operatorname{sgn} \sigma}{\left(x_{1}+\sqrt{-1} \sigma_{1} 0\right) \cdots\left(x_{1}+\sqrt{-1} \sigma_{1} 0\right)} .
\end{aligned}
$$

(c) $(x+\sqrt{-1} 0)^{\lambda}$, with defining function $F_{+}(z)=z^{\lambda}, F_{-}(z)=0$.

(d) For very $\alpha \in \mathbb{Z}$,

$$
\begin{aligned}
& x_{+}^{\alpha}=\left[\frac{-(-z)^{\alpha}}{2 \sqrt{-1} \sin \pi \alpha}\right]_{z=x}, \\
& x_{-}^{\alpha}=\left[\frac{z^{\alpha}}{2 \sqrt{-1} \sin \pi \alpha}\right]_{z=x} .
\end{aligned}
$$

Definition 2.2.7. Let $f \in \mathscr{B}\left(\mathbb{R}^{n}\right)$ be a hyperfunction defined on $\mathbb{R}^{n}$, we say $f$ is micro-analytic at $(x, \sqrt{-1} \xi \infty) \in \sqrt{-1} S^{*} \mathbb{R}^{n}$, i.e., at $(x, \xi) \in \mathbb{R}^{n} \times S^{n-1}$ if $f$ admits a representation as sum of boundary values

$$
f(x)=\sum F_{j}\left(x+\sqrt{-1} \Gamma_{j} 0\right)
$$

with $F_{j}$ holomorphic functions defined on infinitesimal wedges $\mathbb{R}^{n}+i \Gamma_{j} 0$ such that, for any $j=1, \cdots, N$,

$$
\Gamma_{j} \cap\left\{y \in \mathbb{R}^{n}:\langle\xi, y\rangle<0\right\} \neq \emptyset .
$$

The set of all points in $\mathbb{R}^{n} \times \sqrt{-1} S_{\infty}^{n-1}$ (i.e., in $\sqrt{-1} S^{*} \mathbb{R}^{n}$ ) where $f$ is not micro-analytic is called the singular spectrum $3^{3}$ of $f$ and is denoted by S. S. $(f)$.

Example 2.2.8. Let $x=\left(x_{1}, x_{2}, \cdots, x_{n}\right)$ be local coordinate of $\mathbb{R}^{n}$, we have

(a) S.S. $\frac{1}{x_{1}+\sqrt{-1} 0}=\left\{x_{1}=0\right\} \times\left\{\sqrt{-1} d x_{1} \infty\right\}$, where $\sqrt{-1} d x_{1} \infty$ is the vector $\sqrt{-1} d x \infty$ corresponding to $\xi=(1,0, \cdots, 0)$.

\footnotetext{
${ }^{3}$ In $[\mathrm{KKK}][40]$, it is called singularity spectrum.
} 
(b) S.S. $\delta(x)=\{0\} \times S^{n-1}$.

(c) S.S. $\frac{1}{x_{1}-\sqrt{-10}} \frac{1}{x_{2}-\sqrt{-10}}=\left\{x_{1}=0\right\} \times\left\{-\sqrt{-1} d x_{1} \infty\right\} \cup\left\{x_{2}=0\right\} \times$ $\left\{-\sqrt{-1} d x_{2} \infty\right\}$.

From the definition one has

Corollary 2.2.9. A hyperfunction $g \in \mathscr{B}\left(\mathbb{R}^{n}\right)$ is micro-analytic in a neighborhood of $\left(x_{0}, \sqrt{-1} \xi_{0} \infty\right)$ if and only if we have an expression

$$
f(x)=\sum F_{j}\left(x+\sqrt{-1} \Gamma_{j} 0\right)
$$

in a neighborhood of $x_{0}$ with $\Gamma_{j}$ such that

$$
\Gamma_{j} \subset \Gamma_{\xi_{0}} \equiv\left\{y \in \mathbb{R}^{n}:\langle\xi, y\rangle<0\right\}, \quad j=1, \cdots, N .
$$

Definition 2.2.10. Let $M$ be a real analytic manifold, and let $X$ be a complexification of $M$. A function $f(x)$ on $X$ is said to be of positive type if $\operatorname{Re} f(x)=0$ for $x \in M$ implies $\operatorname{Im} f(x) \geq 0$.

Assume $f(x)$ is a complex-valued real analytic function of positive type such that for $x_{0} \in M, f\left(x_{0}\right)=0$ and $d f\left(x_{0}\right)$ is a non-zero real vector. Then $\left\{f^{-1}\left(D_{\epsilon}\right)-M\right\} \cup$ $\sqrt{-1} S M$ is a neighborhood of $x_{0}+\sqrt{-1} v_{0} 0$, where $\left\langle v_{0}, d f\left(x_{0}\right)\right\rangle>0, \epsilon$ is an arbitrary positive real number, and $D_{\epsilon}=\{\tau \in \mathbb{C}: \operatorname{Im} \tau+\epsilon|\operatorname{Re} \tau|>0\}$.

Example 2.2.11. We know

$$
f(x)=x_{1}+\sqrt{-1}\left(x_{2}^{2}+\cdots+x_{n}^{2}\right)
$$

is a complex-valued real analytic function of positive type on $\mathbb{R}^{n}$. Moreover, the hyperfunction $u(x)=(f(x)+\sqrt{-1} 0)^{\varrho}, \varrho \neq 0,1,2 \cdots$ is not real analytic at the origin and contains only one direction as its analytic singularity:

$$
\text { S. S. }(u)=\{(x, \sqrt{-1} \xi d x \infty) \mid x=0, \xi=(1,0, \cdots, 0)\} .
$$

\subsection{Fundamental exact sequences}

Definition 2.3.1. Let $f$ be a hyperfunction on an open subset of $\mathbb{R}^{n}$. The singular support of $f$, denoted by $\operatorname{supp}(f)$, is the complement in $U$ of the largest open subset of $U$ on which $f$ is real analytic.

Remark 2.3.2. Let $\mathcal{A}$ be the sheaf of germs of real analytic functions, let $\mathcal{D}^{\prime}$ be the sheaf of germs of distributions, and let $\mathcal{L}_{1, l o c}$ be the sheaf of locally integrable functions. We have inclusions of sheaves

$$
\mathcal{A} \hookrightarrow \mathcal{L}_{1, l o c} \hookrightarrow \mathcal{D}^{\prime} \hookrightarrow \mathscr{B}
$$


Moreover, the infinite sum

$$
\sum_{m=0}^{\infty} c_{m} \delta^{(m)}, \quad \text { where } c_{m} \in \mathbb{C}, \limsup _{m \rightarrow \infty} \sqrt[m]{\left|c_{m}\right|}=0
$$

represents a hyperfunction with support at $x=0$, but not a distribution unless it is a finite linear combination of $\delta^{(m)}$.

Proposition 2.3.3. (Sato's fundamental sequence) There is an exact sequence of sheaves

$$
0 \longrightarrow \mathcal{A} \longrightarrow \mathscr{B} \stackrel{\text { sp }}{\longrightarrow} \pi_{*} \mathscr{C} \longrightarrow 0
$$

on real analytic manifold $M$.

One can use the above exact sequence to define the sheaf of microfunctions, see Kaneko [34].

Apart from Definition 2.2.7, here is another equivalent definition of analytic singularity.

Definition 2.3.4. For $u \in \mathscr{B}(M)$, the image $\operatorname{sp}(u) \in \pi_{*} \mathscr{C}=\mathscr{C}\left(\sqrt{-1} S^{*} M\right)$ is said to be the spectrum of $u$. The support of $\operatorname{sp}(u)$, denote by S.S. $(u)$, is called the singular spectrum of $u$.

Definition 2.3.5. A subset $Z$ of $\sqrt{-1} S M$ (resp. $\sqrt{-1} S^{*} M$ ) is said to be convex if each fibre $\tau^{-1}(x) \cap Z$ of $\tau: \sqrt{-1} S M \rightarrow M$ (resp. $\tau: \sqrt{-1} S^{*} M \rightarrow M$ ) is convex.

Definition 2.3.6. The polar set $Z^{\circ}$ of a subset $Z \subset \sqrt{-1} S M$ is defined as

$$
Z^{\circ}=\left\{\left(x, \sqrt{-1} \xi_{x} \infty\right) \in \sqrt{-1} S^{*} M \mid\left\langle\xi_{x}, \nu_{x}\right\rangle>0\right\}
$$

for an arbitrary point $x+\sqrt{-1} \nu_{x} 0 \in Z$. The polar set of a subset of $\sqrt{-1} S^{*} M$ is defined similarly.

Definition 2.3.7. Let $D$ be an open set in $X-M$, the open set $D$ is said to be a conoidal neighborhood of $x_{0}+\sqrt{-1} \nu 0$ (of $U \subset \sqrt{-1} S M$ ) if $D \cap \sqrt{-1} S M$ is a neighborhood of $x_{0}+\sqrt{-1} \nu 0$ (of $U$ ). Denote the boundary value of $\varphi \in \mathscr{O}_{X}(D)$ by $b_{D}(\varphi)$.

We denote the hyperfunction corresponding to $\varphi \in \tilde{\mathcal{A}}(U)$ by $b_{U}(\varphi)$ provided that each fibre of $U$ is connected. We also write $b(\varphi ; D)$ and $b(\varphi ; U)$ instead of $b_{D}(\varphi)$ and $b_{U}(\varphi)$ respectively, or even $b(\varphi)$, when there is no fear of confusion.

Note that

$$
\tilde{\mathcal{A}}(U)=\underset{D \in \mathfrak{N}(U)}{\lim _{X}} \mathscr{O}_{X}(D),
$$

where $\mathfrak{N}(U)$ is the set of all conoidal neighborhoods of $U$.

Theorem 2.3.8. Let $M, X$ and $D$ be as before and $U$ be an open set in $\sqrt{-1} S M$ such that each fibre is connected. If $D \in \mathfrak{N}(U)$, then the boundary value of $f(z) \in \mathscr{O}_{X}(D)$ determines a hyperfunction $f(x) \in \mathscr{B}_{M}(\tau(U))$ uniquely and such that $\mathrm{S}$. S. $(f) \subset U^{\circ}$. 
Conversely, if S. S. ( $u)$ of a hyperfunction $u(x)$ on $M$ is contained in a closed convex set $Z \subset \sqrt{-1} S^{*} M$, then there exists a conoidal neighborhood $D \in \mathfrak{N}\left(Z^{\circ}\right)$ such that $u(x)=b_{D}(f(z))$ for some $f(z) \in \mathscr{O}_{X}(D)$.

From the exact sequence

$$
\pi^{-1} \mathscr{B} \stackrel{s p}{\longrightarrow} \mathscr{C} \longrightarrow 0
$$

one has the sequence

$$
0 \longrightarrow \mathcal{A}^{*} \stackrel{b}{\longrightarrow} \pi^{-1} \mathscr{B} \stackrel{s p}{\longrightarrow} \mathscr{C} \longrightarrow 0
$$

is exact, where the sheaf $\mathcal{A}^{*}$ on $\sqrt{-1} S M$ is defined as $\mathcal{A}^{*}=\operatorname{ker}\left(\pi^{-1} \mathscr{B} \rightarrow \mathscr{C}\right)$. Then $u \in \mathcal{A}_{(x, \sqrt{-1} \xi \infty)}^{*}$ can be expressed as

$$
u=\sum_{j} b\left(\varphi_{j}\right)
$$

where $\varphi_{j} \in \Gamma\left(U_{j}, \tilde{\mathcal{A}}\right)$ and $(x, \sqrt{-1} \xi \infty) \notin U_{j}^{\circ}$.

Remark 2.3.9. Roughly speaking, one can define the sheaf of microfunctions $\mathscr{C}$ by considering the quotient sheaf of $\mathscr{B} / \mathcal{A}$.

Definition 2.3.10. In the above case, $u$ is said to be micro-analytic at $(x, \sqrt{-1} \xi \infty)$.

Example 2.3.11. For the Euler operator

$$
P=x \frac{\partial}{\partial x}-\alpha
$$

consider the hyperfunction solutions $u$ of $P u=0$. The dimension of space of hyperfunction solutions is 2 . Choose two linear dependent hyperfunction solutions $(x+\sqrt{-1} 0)^{\alpha}$ and $(x-\sqrt{-1} 0)^{\alpha}$. When $\alpha \neq 0,1, \cdots, n, \cdots$, one has

$$
\text { S. S. }(u) \subset\left\{(0, \pm \sqrt{-1} d x \infty) \in \sqrt{-1} S^{*} \mathbb{R}\right\} .
$$

Otherwise, S. S. $(u)=\emptyset$.

\subsection{Fundamental operations}

In this section we follow [KKK] [40] and introduce the fundamental operations of hyperfunctions and microfunctions, such as production, restriction, substitution, integration, convolution, etc.

Definition 2.4.1. Let $u$ be a hyperfunction on a real analytic manifold $M$, then define

$$
\begin{aligned}
\widehat{\text { S.S. }} u:= & \left\{(x, \sqrt{-1} \xi) \in \sqrt{-1} T^{*} M \mid x \in \operatorname{supp} u \quad \text { and } \quad \xi=0\right\} \\
& \cup\left\{(x, \sqrt{-1} \xi) \in \sqrt{-1} T^{*} M \mid \xi \neq 0 \quad \text { and } \quad(x, \sqrt{-1} \xi \infty) \in \text { S.S. }(u)\right\} .
\end{aligned}
$$


Theorem 2.4.2. Let $M_{1}$ and $M_{2}$ be real analytic manifolds and let $M=M_{1} \times M_{2}$. Let

$$
\left(\sqrt{-1} S^{*} M\right)^{\prime}:=\sqrt{-1} S^{*} M-\sqrt{-1} S^{*} M_{1} \times M_{2}-M_{1} \times \sqrt{-1} S^{*} M_{2},
$$

and define two projections

$$
\begin{aligned}
p_{1}:\left(\sqrt{S^{*} M}\right)^{\prime} & \rightarrow \sqrt{-1} S^{*} M_{1} \\
\left(\left(x_{1}, x_{2}\right), \sqrt{-1}\left(\xi_{1}, \xi_{2}\right) \infty\right) & \mapsto\left(x_{1}, \sqrt{-1} \xi_{1} \infty\right), \\
p_{2}:\left(\sqrt{S^{*} M}\right)^{\prime} & \rightarrow \sqrt{-1} S^{*} M_{2} \\
\left(\left(x_{1}, x_{2}\right), \sqrt{-1}\left(\xi_{1}, \xi_{2}\right) \infty\right) & \mapsto\left(x_{2}, \sqrt{-1} \xi_{2} \infty\right) .
\end{aligned}
$$

For an open subset $\Omega_{j} \in \sqrt{-1} S^{*} M_{j}, j=1,2$, let

$\Omega_{1} \widehat{\times} \Omega_{2}:=\left\{\left(\left(x_{1}, x_{2}\right), \sqrt{-1}\left(\xi_{1}, \xi_{2}\right) \infty\right) \mid \xi_{1}^{2}+\xi_{2}^{2} \neq 0\right.$ and $\left(x_{j}, \sqrt{-1} \xi_{j} \infty\right) \in \Omega_{j}$ for $\left.j=1,2\right\}$.

Then we have

(a) For two hyperfunctions $u_{1}\left(x_{1}\right) \in \mathscr{B}\left(M_{1}\right)$ and $u_{2}\left(x_{2}\right) \in \mathscr{B}\left(M_{2}\right)$, one can define canonically the product $u\left(x_{1}, x_{2}\right):=u_{1}\left(x_{1}\right) u_{2}\left(x_{2}\right)$ so that

$$
\widehat{\text { S.S. }}(u) \subset \widehat{\text { S.S. }} .\left(u_{1}\right) \times \widehat{\text { S.S. }} .\left(u_{2}\right) .
$$

That is, there is a canonical sheaf homomorphism

$$
\mathscr{B}_{M_{1}} \times \mathscr{B}_{M_{2}} \rightarrow \mathscr{B}_{M}
$$

(b) For two microfunctions $u_{1}\left(x_{1}\right) \in \mathscr{C}_{M_{1}}\left(\Omega_{1}\right)$ and $u_{2}\left(x_{2}\right) \in \mathscr{C}_{M_{2}}\left(\Omega_{2}\right)$, one can define the product $u\left(x_{1}, x_{2}\right):=u_{1}\left(x_{1}\right) u_{2}\left(x_{2}\right) \in \mathscr{C}_{M}\left(\Omega_{1} \widehat{\times} \Omega_{2}\right)$. That is, there is a canonical sheaf homomorphism

$$
p_{1}^{-1} \mathscr{C}_{M_{1}} \times\left. p_{2}^{-2} \mathscr{C}_{M_{2}} \rightarrow \mathscr{C}_{M}\right|_{\left(\sqrt{-1} S^{*} M\right)^{\prime}}
$$

Definition 2.4.3. Let $N$ and $M$ be real analytic manifolds, and let $f: N \rightarrow M$ be a real analytic map. For $y \in N$ and $\xi \in T_{f(y)}^{*} M$, define a map

$$
\begin{aligned}
\hat{\rho}: N \times_{M} T^{*} M & \rightarrow T^{*} N \\
(y, \xi) & \mapsto\left(y, f^{*}(\xi)\right) .
\end{aligned}
$$

The kernel of $\hat{\rho}$, denoted by $T_{N}^{*} M$, is the conormal bundle with supports in $N$.

Since $N=\left\{(y, \xi) \in N \times{ }_{M} T^{*} M \mid y \in N\right.$ and $\left.\xi=0\right\} \in T_{N}^{*} M$, we denote $S_{N}^{*} M:=$ $\left(T_{N}^{*} M-N\right) / \mathbb{R}_{+}^{\times}$. Moreover, notice that $\sqrt{-1} S_{N}^{*} M$ is a closed set in $N \times_{M} \sqrt{-1} S^{*} M$, 
we can define two maps as follows:

$$
\begin{aligned}
\rho=\rho_{f}: N \times_{M} \sqrt{-1} S^{*} M-\sqrt{-1} S_{N}^{*} M & \rightarrow \sqrt{-1} S^{*} N \\
(y, \sqrt{-1} \xi \infty) & \mapsto\left(y, \sqrt{-1} f^{*}(\xi) \infty\right), \\
\varpi=\varpi_{f}: N \times_{M} \sqrt{-1} S^{*} M-\sqrt{-1} S_{N}^{*} M & \rightarrow \sqrt{-1} S^{*} M \\
(y, \sqrt{-1} \xi \infty) & \mapsto(f(y), \sqrt{-1} \xi \infty) .
\end{aligned}
$$

Theorem 2.4.4. Let $N$ be a submanifold of $M$, and $\iota$ be the embedding $N \hookrightarrow M$.

(a) (Restriction of a hyperfunction) Let $u$ be a hyperfunction on $M$ such that S.S. $(u) \cap \sqrt{-1} S_{N}^{*} M=\emptyset$. Then one can define the restriction of $u$ to $N$, $\left.u\right|_{N} \in \mathscr{B}_{N}$, such that

$$
\begin{aligned}
& \widehat{\text { S.S. }}\left(\left.u\right|_{N}\right) \subset \hat{\rho}\left(N \times_{M} \sqrt{-1} T^{*} M \cap \widehat{\text { S.S. }} .(u)\right), \\
& \text { S. S. }\left(\left.u\right|_{N}\right) \subset \rho\left(N \times_{M} \sqrt{-1} T^{*} M \cap \text { S. S. }(u)\right)
\end{aligned}
$$

(b) (Restriction of a microfunction) There exists a sheaf homomorphism

$$
\rho ! \varpi^{-1} \mathscr{C}_{M} \rightarrow \mathscr{C}_{N}
$$

Example 2.4.5. $\quad$ (a) $\left.\delta\left(x_{1}-x_{2}\right)\right|_{x_{2}=0}=\delta\left(x_{1}\right)$.

(b) Let $h$ and $f$ be real-valued real analytic functions defined in an open subset $U \subset \mathbb{R}^{n}$, and let $\Omega=\{x \in U \mid h(x)=0\}$ be a non-singular hypersurface, i.e., $d h \neq 0$ on $\Omega$. Moreover, assume $\nabla_{x} h(x)$ and $\nabla_{x} f(x)$ are linear independent on $\Omega \cap\{f=0\}$. Then the restriction of $\delta(f(x))$ to $\Omega$ is well defined as an element of of $\mathscr{B}_{\Omega}(\Omega)$ so that $\left(\left.f\right|_{\Omega}\right)^{*} \delta(t)=\delta\left(\left.f(x)\right|_{\Omega}\right)$. From the exact sequence

$$
0 \longrightarrow T_{\Omega}^{*} U \longrightarrow T^{*} U \times_{U} \Omega \longrightarrow T^{*} \Omega \longrightarrow 0,
$$

a point on $T^{*} \Omega$ can be described as a point on $T^{*} U \cong U \times \mathbb{R}^{n}$ module the set $T_{\Omega}^{*} U$, then

$$
\text { S. S. } \delta\left(\left.f(x)\right|_{\Omega}\right)=A \quad \text { module } B \text {, }
$$

where

$$
\begin{aligned}
& A=\left\{(x, \sqrt{-1} \xi) \in U \times \sqrt{-1} \mathbb{R}^{n} \mid f(x)=h(x)=0\right. \text { and } \\
&\left.\xi=c_{1} \nabla_{x} f(x)+c_{2} \nabla_{x} h(x), c_{1}, c_{2} \in \mathbb{R}, c_{1} \neq 0\right\}, \\
& B=\left\{(x, \sqrt{-1} \xi) \in U \times \sqrt{-1} \mathbb{R}^{n} \mid h(x)=0 \text { and } \xi=c \nabla_{x} h(x), c \in \mathbb{R}\right\} .
\end{aligned}
$$

Theorem 2.4.6. Let $M$ be real analytic manifold, and let $\Delta_{M}$ be the diagonal set of $M \times M$.

(a) (Product of hyperfunctions) Let $u(x)$ and $v(x)$ be hyperfunctions on $M$ such that 
S. S. $(u) \cap(\text { S. S. }(v))^{a}=\emptyset$, where

$$
\begin{aligned}
a: \sqrt{-1} S^{*} M & \rightarrow \sqrt{-1} S^{*} M \\
(x, \sqrt{-1} \xi \infty) & \mapsto(x,-\sqrt{-1} \xi \infty)
\end{aligned}
$$

is the antipodal mapping and (S.S. $(v))^{a}$ denotes the image of S. S. $(v)$ under a. Then the product $u(x) v(x) \in \mathscr{B}(M)$ exists, such that

$\widehat{\text { S.S. }}(u v) \subset\left\{\left(x, \sqrt{-1}\left(\xi_{1}+\xi_{2}\right)\right) \mid\left(x, \sqrt{-1} \xi_{1}\right) \in \widehat{S . S} . u\right.$ and $\left.\left(x, \sqrt{-1} \xi_{2}\right) \in \widehat{\text { S.S. }} .(v)\right\}$,

$$
\begin{gathered}
\text { S.S. }(u v) \subset\left\{\left(x, \sqrt{-1}\left(\theta \xi_{1}+(1-\theta) \xi_{2}\right) \infty\right) \mid\right. \\
\left.\left(x, \sqrt{-1} \xi_{1} \infty\right) \in \text { S. S. }(u),\left(x, \sqrt{-1} \xi_{2} \infty\right) \in \text { S. S. }(v) \text { and } 0 \leq \theta \leq 1\right\} \cup \operatorname{S} . \text { S. }(u) \cup \operatorname{S} . \text { S. }(v) .
\end{gathered}
$$

(b) (Product of microfunctions) We define

$$
\begin{gathered}
N:=\Delta_{M} \times_{M \times M}\left(\sqrt{-1} S^{*}(M \times M)\right)-\Delta_{M} \times_{M \times M}\left(M \times \sqrt{-1} S^{*} M\right) \\
-\Delta_{M} \times_{M \times M}\left(\sqrt{-1} S^{*} M \times M\right)-\sqrt{-1} S^{*}(M \times M) .
\end{gathered}
$$

For a point $z=\left(x, x, \sqrt{-1}\left(\xi_{1}, \xi_{2}\right) \infty\right) \in N$, where $\xi_{1} \neq 0, \xi_{2} \neq 0$ and $\xi_{1}+$ $\xi_{2} \neq 0$, we let $p_{1}(z)=\left(x, \sqrt{-1} \xi_{1} \infty\right) \in \sqrt{-1} S^{*} M, p_{2}(z)=\left(x, \sqrt{-1} \xi_{2} \infty\right) \in$ $\sqrt{-1} S^{*} M$, and $q(z)=\left(x, \sqrt{-1}\left(\xi_{1}+\xi_{2}\right) \infty\right) \in \sqrt{-1} S^{*} M$, then there is a sheaf homomorphism

$$
q_{!}\left(p_{1}^{-1} \mathscr{C}_{M} \times p_{2}^{-2} \mathscr{C}_{M}\right) \rightarrow \mathscr{C}_{M}
$$

Example 2.4.7. (a) Let $f(x)$ and $g(x)$ be real-valued real analytic functions defined in $U \subset \mathbb{R}^{n}$, satisfying that

- if $f(x)=0$, then $\nabla_{x} f(x) \neq 0$,

- if $f(x)=0$, then $\nabla_{x} g(x) \neq 0$,

- on the set $\{f(x)=g(x)=0\}, \nabla_{x} f(x)$ and $\nabla_{x} g(x)$ are linear independent.

Then the product $\delta(f(x)) \delta(g(x))$ is well defined, and we have

$$
\begin{gathered}
\text { S.S. }(\delta(f) \delta(g)) \subset\left\{(x, \sqrt{-1} \xi \infty) \in \sqrt{-1} S^{*} U \mid f(x)=g(x)=0 \quad\right. \text { and } \\
\left.\xi=c_{1} \nabla_{x} f(x)+c_{2} \nabla_{x} g(x),\left(c_{1}, c_{2}\right) \in \mathbb{R}^{2} \backslash 0\right\} .
\end{gathered}
$$

(b) The products

$$
\frac{1}{x_{1}+\sqrt{-1} 0} \cdot \frac{1}{x_{1}-x_{2}^{2}+\sqrt{-1} 0} \text { and } \frac{1}{\left(x_{1}+\sqrt{-1} 0\right)^{2}}
$$

are well defined. However, the products $\delta\left(x_{1}\right) \delta\left(x_{1}-x_{2}^{2}\right)$ and $\delta\left(x_{2}\right)^{2}$ are not well defined. 
Theorem 2.4.8. Let $N$ and $M$ be real analytic manifolds, and let $f: N \rightarrow M$ be a smooth real analytic map.

(a) (Substitution for hyperfunctions) There induced a sheaf homomorphism

$$
f^{*}: f^{-1} \mathscr{B}_{M} \rightarrow \mathscr{B}_{N}
$$

Furthermore, define

$$
\begin{aligned}
\hat{\rho}: N \times_{M}\left(\sqrt{-1} T^{*} M\right) & \rightarrow \sqrt{-1} T^{*} N \\
(y, \sqrt{-1} \xi) & \mapsto\left(y, \sqrt{-1} f^{*}(\xi)\right), \\
\hat{\varpi}: N \times_{M}\left(\sqrt{-1} T^{*} M\right) & \rightarrow \sqrt{-1} T^{*} M \\
(y, \sqrt{-1} \xi) & \mapsto(f(y), \sqrt{-1} \xi),
\end{aligned}
$$

and $\rho, \varpi$ as before, then

$$
\begin{aligned}
& \widehat{\text { S.S. }} \cdot\left(f^{*} u\right)=\hat{\rho} \hat{\varpi}^{-1}(\widehat{\mathrm{S} . \mathrm{S}} \cdot(u)) \\
& \text { S.S. }\left(f^{*} u\right)=\rho \varpi^{-1}(\mathrm{~S} . \mathrm{S} .(u))
\end{aligned}
$$

(b) (Substitution for microfunctions) There is a sheaf homomorphism

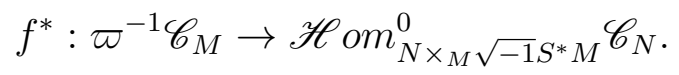

Example 2.4.9. Let $f(x)$ be a real-valued real analytic function defined in an open subset $U \subset \mathbb{R}^{n}$, assume that $f(x)=0$ implies $d_{x} f(x) \neq 0$. Then the substitution $f^{*}(1 /(t+\sqrt{-1} 0))$ is well defined, denoted by $1 /(f(x)+\sqrt{-1} 0)$, and

S.S. $\left(\frac{1}{f(x+\sqrt{-1} 0)}\right) \subset\left\{(x, \sqrt{-1} \xi \infty) \in \sqrt{-1} S^{*} U \mid f(x)=0\right.$ and $\left.\xi=c \nabla_{x} f(x), c>0\right\}$.

Theorem 2.4.10. Let $N$ and $M$ be real analytic manifolds.

(a) (Integration of hyperfunction) Let $f: M \times N \rightarrow N$ and $\pi: M \times$ $\sqrt{-1} S^{*} N \rightarrow \sqrt{-1} S^{*} N$ be the natural projections. If $\left.f\right|_{\text {supp } u}$ is a proper map for a hyperfunction $u(t, x)$ on $M \times N$, then the integration of $u(t, x)$ along the fiber

$$
v(x)=\int_{f^{-1}(x)} u(t, x) d t
$$

can be defined. Moreover, one has

$$
\text { S. S. }(v(x)) \subset \pi\left(\text { S. S. }(u) \cap M \times \sqrt{-1} S^{*} N\right),
$$

That is, there exists a sheaf homomorphism

$$
f_{!}\left(\mathscr{B}_{M \times N} \otimes v_{M}\right) \rightarrow \mathscr{B}_{N},
$$

where $v_{M}=\Omega_{M}^{n} \otimes \omega_{M}, \Omega_{M}^{n}$ is a sheaf of holomorphic differential forms of degree 
$n$ on $M$ of dimension $m$ and $\omega_{M}$ is the orientation sheaf on $M$.

(b) (Integration of microfunction) Let $\pi$ be as before and let $U$ be an open subset of $\sqrt{-1} S^{*} N$. For $u(t, x) \in \mathscr{C}_{M \times N}\left(\pi^{-1}(U)\right)$, if $\left.\pi\right|_{\operatorname{supp} u}$ is a proper map, then the integration

$$
v(x)=\int_{\pi^{-1}(x)} u(t, x) d t
$$

is well-defined as a microfunction. There exists a sheaf homomorphism

$$
\pi_{!}:\left(\left.\mathscr{C}_{M \times N}\right|_{M \times \sqrt{-1} S^{*} N} \otimes v_{M}\right) \rightarrow \mathscr{C}_{N}
$$

Example 2.4.11. (a) $\int_{\mathbb{R}} \delta(t) d t=1$.

(b) $\int_{\mathbb{R}} \delta^{(n)}(x) d x=0$ for $n \geq 1$, where $\delta^{(n)}(x)=\frac{d^{n}}{d x^{n}} \delta(x)$.

(c) $\int_{-\infty}^{1}(\lambda+1) x_{+}^{\lambda} d x=1$ for $-\lambda \notin \mathbb{Z}_{+}$.

(d) $\int_{\mathbb{R}} \delta\left(t-x^{2}\right) d x=t_{+}^{-1 / 2}$.

(e) $\frac{1}{2 \pi \sqrt{-1}} \int_{-\pi}^{\pi} \frac{d \theta}{\cos \theta+\sqrt{-10}}=1$.

(f) $\int_{\mathbb{R}} \delta\left(x_{1}^{2}+\cdots+x_{n}^{2}-t\right)_{-}^{\lambda} d x_{1} \cdots d x_{n}=\frac{\pi^{n / 2} \Gamma(\lambda+1)}{\Gamma(\lambda+n / 2+1)} t_{+}^{\lambda+n / 2}$.

Definition 2.4.12. Let $f(x)$ and $g(x)$ be two hyperfunctions on $\mathbb{R}^{n}$, at least one of the which has compact support. The convolution of $f$ and $g$ is defined as

$$
f * g=\int_{\mathbb{R}^{n}} f(x-y) g(y) d y .
$$

Proposition 2.4.13. The following equations hold.

$$
\begin{gathered}
f * g=g * f ; \\
D^{\alpha}(f * g)=\left(D^{\alpha} f\right) * g=f *\left(D^{\alpha} g\right) ; \\
\delta * f=f * g=f .
\end{gathered}
$$

In addition, if $h$ also has compact supported, then

$$
(f * g) * h=f *(g * h) .
$$

Besides, the following estimates are valid.

$$
\operatorname{supp} f * g \subset \operatorname{supp} f+\operatorname{supp} g \text {. }
$$

$\operatorname{sing} \operatorname{supp} f * g \subset \operatorname{sing} \operatorname{supp} f+\operatorname{sing} \operatorname{supp} g$.

$$
\begin{array}{r}
\text { S. S. }(f * g) \subset\{(x+y ; \sqrt{-1} \xi d x \infty) \mid(x, \sqrt{-1} \xi d x \infty) \in \text { S.S. }(f), \\
(y, \sqrt{-1} \xi d x \infty) \in \text { S.S. }(g)\} .
\end{array}
$$


Remark 2.4.14. The convolution $f * g$ can be well defined as long as the integral (2.2) make sense as a hyperfunction in $x$, henceforth there are many other cases aside from Definition 2.4.12 in which convolution is well defined. One can see C.5.4 in Appendix C for another generalized definition.

\subsection{Sheaf $\mathcal{E}_{X}$ of microdifferential operators}

For practical manipulation, we introduce the class of microdifferential operators in this section, which is located between the class of microlocal operators and the class of differential operators. Roughly, the class of microdifferential operators is a special case of microlocal operators of which the inverse exist.

Definition 2.5.1. Let $M$ and $N$ be real analytic manifolds, and let $Z$ be a locally closed subset of $\sqrt{-1} S^{*}(M \times N)$ such that $Z \cap M \times \sqrt{-1} S^{*} N=\emptyset$ and $Z \cap \sqrt{-1} S^{*} M \times N=\emptyset$. Furthermore, one define two projections

$$
\begin{aligned}
p_{1}: \sqrt{-1} S^{*}(M \times N)-\sqrt{-1} S^{*} M \times N-M \times \sqrt{-1} S^{*} N & \rightarrow \sqrt{-1} S^{*} M \\
((x, y), \sqrt{-1}(\xi, \eta) \infty) & \mapsto(x, \sqrt{-1} \xi \infty), \\
p_{2}: \sqrt{-1} S^{*}(M \times N)-\sqrt{-1} S^{*} M \times N-M \times \sqrt{-1} S^{*} N & \rightarrow \sqrt{-1} S^{*} M_{2} \\
((x, y), \sqrt{-1}(\xi, \eta) \infty) & \mapsto(y, \sqrt{-1} \eta \infty) .
\end{aligned}
$$

Assume $p_{1}(Z)$ is locally closed subset of $\sqrt{-1} S^{*} M$, and denote the sheaf of volume elements of $N$ by $v_{N}$, then define an integral operator $\mathscr{K}$ as

$$
\mathscr{K}(u)=\int_{N} K(x, y) u(y) d y
$$

for $K(x, y) \in H_{Z}^{0}\left(\sqrt{-1} S^{*}(M \times N), \mathscr{C}_{M \times N} \otimes v_{N}\right)$, and $u \in \mathscr{C}_{N}$. Hence one obtains a sheaf homomorphism

$$
\mathscr{K}:\left(\left.p_{1}\right|_{Z}\right) !\left(\left.p_{2}^{a}\right|_{Z}\right)^{-1} \mathscr{C}_{N} \rightarrow \mathscr{C}_{M}
$$

where $a$ is the antipodal map.

From the above definition, for an element

$$
K(x, y) d y \in \mathscr{H}_{\sqrt{-1} S_{M}^{*}(M \times M)}^{0}\left(\mathscr{C}_{M \times M}\right),
$$

the integral operator $\mathscr{K}(u)=\int K(x, y) u(y) d y$ defines a sheaf homomorphism from $\mathscr{C}_{M}$ to $\mathscr{C}_{M}$.

Definition 2.5.2. The sheaf $\mathscr{H}_{\sqrt{-1} S_{M}^{*}(M \times M)}^{0}\left(\mathscr{C}_{M \times M} \otimes v_{M}\right)$ is said to be the sheaf of microlocal operators and denoted by $\mathcal{L}_{M}$.

Definition 2.5.3. Let $P\left(x, \partial_{x}\right)=\sum_{|\alpha| \leq m} a_{\alpha}(x) \partial_{x}^{\alpha}$ be a linear differential operator of order $m$, where $\partial_{x}^{\alpha}=\frac{\partial^{|\alpha|}}{\partial x_{1}^{\alpha_{1} \ldots \partial x_{n}^{\alpha_{n}}}} 4^{4} \alpha=\left(\alpha_{1}, \cdots, \alpha_{n}\right) \in\left(\mathbb{Z}^{+} \sqcup\{0\}\right)^{n}$ and

\footnotetext{
${ }^{4}$ in [KKK] [40], it claims $D_{x}=\frac{\partial}{\partial x}$, and then the principal symbol is $\sigma(P)(x, \sqrt{-1} \xi)$. I will follow
} 
$a_{\alpha}(x)$ are holomorphic functions. It is clearly a microlocal operator with the kernel function $P\left(x, \partial_{x}\right) \delta(x-y) d y$. The principal symbol $\sigma(P)(x, \sqrt{-1} \xi)$ is defined as $\sigma(P)(x, \xi)=\sum_{|\alpha|=m} a_{\alpha}(x)(\sqrt{-1} \xi)^{\alpha} \in \mathscr{O}_{T^{*} M}$. It does not depend on the choice of the local coordinate systems.

Sato's fundamental theorem is a consequence of the Cauchy-Kovalevsky theorem.

Theorem 2.5.4. (M. Sato) A linear differential operator of finite order $P\left(x, \partial_{x}\right)$ is leftand right-invertible in the ring $\mathscr{L}_{M}$ over

$$
\left\{(x, \sqrt{-1} \xi \infty) \in \sqrt{-1} S^{*} M \mid \sigma(P)(x, \sqrt{-1} \xi) \neq 0\right\} .
$$

Definition 2.5.5. A linear differential operator $P\left(x, \partial_{x}\right)$ is said to be an elliptic operator at $x_{0}$ if for an arbitrary $\xi \in \mathbb{R}^{n}-\{0\}, \sigma(P)\left(x_{0}, \sqrt{-1} \xi\right) \neq 0$ holds.

Theorem 2.5.6. We have

(a) if hyperfunctions $f(x)$ and $u(x)$ satisfy $P\left(x, \partial_{x}\right) u(x)=f(x)$, then one has

$$
\text { S. S. }(u) \subset\left\{(x, \sqrt{-1}\langle\xi, d x\rangle \infty) \in \sqrt{-1} S^{*} M \mid \sigma(P)(x, \sqrt{-1} \xi)=0\right\} \cup \operatorname{S.S} .(f) .
$$

Specially, if $P\left(x, \partial_{x}\right)$ is elliptic at arbitrary point in $M$, then $f(x)$ is real analytic in $M$ implies $u(x)$ is also real analytic in $M$. More precisely, if we denote by $\mathcal{A}$ the sheaf of real analytic functions on $M$ and by $\mathscr{B}\left(\right.$ resp. $\left.\mathscr{D}^{\prime}\right)$ the sheaf of hyperfunctions (resp. distributions) on $M$, then $P: \mathscr{B} / \mathcal{A} \rightarrow \mathscr{B} / \mathcal{A}$ (resp. $P$ : $\left.\mathscr{D}^{\prime} / \mathcal{A} \rightarrow \mathscr{D}^{\prime} / \mathcal{A}\right)$ is a sheaf isomorphism.

(b) If $P\left(x, \partial_{x}\right)$ is elliptic at $x_{0}$, then $P: \mathscr{B}_{x_{0}} \rightarrow \mathscr{B}_{x_{0}}$ is an epimorphism, where $\mathscr{B}_{x_{0}}$ is the sheaf of hyperfunctions with support at $x_{0}$.

For $f(x) \in \mathcal{A}(M)$, a solution $u(x)$ of $P\left(x, \partial_{x}\right) u(x)=f(x)$ has the singular spectrum in

$$
\left\{(x, \sqrt{-1} \xi \infty) \in \sqrt{-1} S^{*} M \mid \sigma(P)(x, \sqrt{-1} \xi)=0\right\},
$$

and the zeros set is called characteristic variety. Study and analysis such set is one of the most important goal in the theory of linear differential equations. The central problem has been recognized in the case of equations with constant coefficients (Ehrenpreis [17]). In the case of variable coefficients, Hömander [29] and Mizohata [60] had shown some results, and the theory of microfunctions is of great advantage to consider this problem.

The class of micro-local operator $\mathcal{L}_{M}$ is too general to manipulate the algebraic properties. A desired class for algebraic consideration is the microdifferential operators.

Let $X$ be an n-dimensional complex manifold and let $\pi_{X}: T^{*} X \rightarrow X$ be the cotangent bundle of $X$. Take a local coordinate system $\left(x_{1}, \cdots, x_{n}\right)$ of $X$ and the associated coordinates $\left(x_{1}, \cdots, x_{n} ; \xi_{1}, \cdots, \xi_{n}\right)$ of $T^{*} X$. Let $\left\{P_{j}(x, \xi)\right\}$ and $\left\{Q_{j}(x, \xi)\right\}$

their notation in this chapter. However, in the last two chapters, we follow the modern way of definition $D_{x}=\frac{1}{\sqrt{-1}} \frac{\partial}{\partial x}$. 
be the total symbol of the differential operator $P=\sum P_{j}(x, \partial)$ and $Q=\sum Q_{j}(x, \partial)$, respectively. Set $S=P+Q$ and $R=P Q$, then the total symbols $\left\{S_{j}\right\}$ and $\left\{R_{j}\right\}$ of $R$ and $S$ are given by

$$
\begin{gathered}
S_{j}=P_{j}+Q_{j} \\
R_{l}=\sum_{l=j+k-|\alpha|}, \alpha \in \mathbb{N}^{n} \frac{1}{\alpha !}\left(\partial_{\xi}^{\alpha} P_{j}\right)\left(\partial_{x}^{\alpha} Q_{k}\right),
\end{gathered}
$$

where $\partial_{\xi}^{\alpha}=\left(\partial / \partial \xi_{1}\right)^{\alpha_{1}} \cdots\left(\partial / \partial \xi_{n}\right)^{\alpha_{n}}$ and $\partial_{x}^{\alpha}=\left(\partial / \partial x_{1}\right)^{\alpha_{1}} \cdots\left(\partial / \partial x_{n}\right)^{\alpha_{n}}$.

The total symbol $\left\{P_{j}(x, \xi)\right\}$ of a differential operator is a polynomial in $\xi$. We shall define microdifferential operator by admitting $P_{j}$ to be holomorphic in $\xi$. The class of microdifferential operators is between the class of microlocal operators and the class of differential operators.

For $\lambda \in \mathbb{C}$, let $\mathscr{O}_{T^{*} X}(\lambda)$ be the sheaf of homogeneous holomorphic functions of degree $\lambda$ on $T^{*} X$, i.e., holomorphic functions $f(x, \xi)$ satisfying

$$
\left(\sum_{j} \xi_{j} \frac{\partial}{\partial \xi_{j}}-\lambda\right) f(x, \xi)=0 .
$$

Definition 2.5.7. For $\lambda \in \mathbb{C}$ we define the sheaf $\mathcal{E}_{X}(\lambda)$ of microdifferential operators of order equal or less then $\lambda$ on $T^{*} X$ by

$$
\begin{gathered}
\Omega \mapsto\left\{\left(P_{\lambda-j}(x, \xi)\right)_{j \in \mathbb{N}} ; P_{\lambda-j} \in \Gamma\left(\Omega ; \mathscr{O}_{T^{*} X}(\lambda-j)\right)\right. \\
\text { and satisfies the following conditions } \left.\left(^{*}\right)\right\}
\end{gathered}
$$

(*) for any compact subset $K$ of $\Omega$, there exists a $C_{k}>0$ such that

$$
\sup _{K}\left|P_{\lambda-j}\right| \leq C_{K}^{-j}(j !) \text { for all } j>0 .
$$

Remark 2.5.8. The growth condition (*) can be explained as follows. For a differential operator $P=\sum P_{j}(x, \partial)$, we have

$$
P(x, \partial)(\langle x, \xi\rangle+p)^{\mu}=\sum P_{j}(x, \xi) \frac{\Gamma(\mu)}{\Gamma(\mu-j+1)}(\langle x, \xi\rangle+p)^{\mu-j} .
$$

For $P=P_{\lambda-j}(x, \xi) \in \mathcal{E}(\lambda)$, by analogy we set

$$
P(\langle x, \xi\rangle+p)^{\mu}=\sum_{j} P_{\lambda-j}(x, \xi) \frac{\Gamma(\mu)}{\Gamma(\mu-\lambda+j+1)}(\langle x, \xi\rangle+p)^{\mu-\lambda+j} .
$$

Then the growth condition $(*)$ is simply the condition that the right hand side converges when $0<|\langle x, \xi\rangle+p| \ll 1$.

Proposition 2.5.9. For $P \in \mathcal{E}(m)$ and $Q \in \mathcal{E}(n)$, set

$$
[P, Q]=P Q-Q P \in \mathcal{E}(m+n-1),
$$


then

$$
\sigma_{m+n-1}([P, Q])=\left\{\sigma_{m}(P), \sigma_{n}(Q)\right\} .
$$

Proposition 2.5.10. ([SKK] [63], Chapter 2) We have

(1) $\mathcal{E}_{X}(\lambda)$ contains $\mathcal{E}_{X}(\lambda-m)$ as a subsheaffor $m \in \mathbb{N}$.

(2) $\mathcal{E}_{X}(\lambda)$ can be defined globally on $T^{*} X$, by patching local pieces.

(3) $\mathcal{E}_{X}(\lambda)$ is a sheaf of $\mathbb{C}$-vector space on $T^{*} X$.

(4) Use the formula (2.4), one can define a homomorphism

$$
\mathcal{E}_{X}(\lambda) \otimes_{\mathbb{C}} \mathcal{E}_{X}(\mu) \rightarrow \mathcal{E}_{X}(\lambda+\mu) .
$$

(5) $\mathcal{E}_{X}(0)$ and $\mathcal{E}_{X}=\cap_{m \in \mathbb{Z}} \mathcal{E}_{X}(m)$ become sheaves of non commutative rings on $T^{*} X$ with a unit.

We define the homomorphism

$$
\begin{aligned}
\sigma_{\lambda}: \mathcal{E}_{X}(\lambda) & \rightarrow \mathscr{O}_{T^{*} X}(\lambda) \\
\left(P_{\lambda-j}\right) & \mapsto P_{\lambda} .
\end{aligned}
$$

Then $\sigma_{\lambda}$ is well-defined on $T^{*} X$ (i.e., compatible with coordinate transformation) and we have an exact sequence

$$
0 \longrightarrow \mathcal{E}_{X}(\lambda-1) \longrightarrow \mathcal{E}_{X}(\lambda) \stackrel{\sigma_{\lambda}}{\longrightarrow} \mathscr{O}_{T^{*} X}(\lambda) \longrightarrow 0
$$

Proposition 2.5.11. We have the following proposition, which says that the ring $\mathcal{E}_{X}$ is a kind of localization of $\mathcal{D}_{X}$.

(1) For $P \in \mathcal{E}(\lambda)$ and $Q \in \mathcal{E}(\mu)$, we have $\sigma_{\lambda+\mu}(P Q)=\sigma_{\lambda}(P) \sigma_{\mu}(Q)$.

(2) ([SKK]) If $P \in \mathcal{E}(\lambda)$ satisfies $\sigma_{\lambda}(P)(q) \neq 0$ at $q \in T^{*} X$, then there exists $Q \in \mathcal{E}_{X}(-\lambda)$ such that $P Q=Q P=1$.

Theorem 2.5.12. The relations between $\mathcal{E}_{X}$ and $\mathcal{D}_{X}$ are summarized as following:

(1) $\mathcal{E}_{X}$ contains $\pi^{-1} \mathcal{D}_{X}$ as a subring and is flat $5^{5}$ over $\pi^{-1} \mathcal{D}_{X}$.

(2) $\left.\mathcal{E}_{X}\right|_{T_{X}^{*} X} \simeq \mathcal{D}_{X}$, where $T_{X}^{*} X$ is the zero section of $T^{*} X$.

(3) For a coherent $\mathcal{D}_{X}$-module $\mathscr{M}$, the characteristic variety of $\mathscr{M}$ coincides with the support of $\mathcal{E}_{X} \otimes_{\pi_{X}^{-1} \mathcal{D}_{X}} \pi_{X}^{-1} \mathscr{M}$.

\footnotetext{
${ }^{5}$ Let $M$ be a R-module. For two R-modules $A$ and $B$, if $f: A \rightarrow B$ is injective implies that $M \otimes_{R} A \rightarrow M \otimes_{R} B$ is injective, we say $M$ is flat over $R$.
} 
Definition 2.5.13. The sheaf $\widehat{\mathcal{E}}_{X}=\lim _{m \in \mathbb{N}} \mathcal{E}_{X} / \mathcal{E}_{X}(-m)$ is called the sheaf of formal micro-differential operators. It is the sheaf similar to $\mathcal{E}$ by dropping the growth condition $(*)$.

More specifically, let $\Omega$ be a subset of $T^{*} X$, denote by $\widehat{\mathcal{E}}_{X}(m)(\Omega)$ the space of formal series:

$$
P=\sum_{-\infty<j \leq m} p_{j}
$$

where $p_{j}$ is a section of $\mathscr{O}_{T^{*} X}(j)$ on $U$. The correspondence

$$
U \rightarrow\left\{\hat{\mathcal{E}}_{X}(m)(\Omega)\right\}
$$

defines a sheaf, denoted $\widehat{\mathcal{E}}_{X}(m)$, and set

$$
\widehat{\mathcal{E}}_{X}=\bigcup_{m} \widehat{\mathcal{E}}_{X}(m)
$$

Definition 2.5.14. The sheaf $\mathcal{E}_{X}^{\infty}$ of micro-differential operators of infinite order can be defined as

$$
\begin{gathered}
\Gamma\left(\Omega ; \mathcal{E}^{\infty}\right) \mapsto\left\{\left(p_{j}\right)_{j \in \mathbb{Z}} ; P_{j} \in \Gamma\left(\Omega ; \mathscr{O}_{T^{*} X}(j)\right)\right. \\
\text { satisfying the following conditions (a) and (b) }\} \text {. }
\end{gathered}
$$

(a) For any compact subset $K$ of $\Omega$, there is a $C_{K}>0$ such that $\sup _{K}\left|p_{j}\right| \leq$ $C_{K}^{-j}(-j)$ ! for $j<0$.

(b) For any compact subset $K$ of $\Omega$ and any $\varepsilon>0$, there exists a $C_{K, \varepsilon}>0$ such that $\sup _{K}\left|p_{j}\right| \leq C_{K, \varepsilon} \frac{\varepsilon^{j}}{j !}$ for $j \geq 1$.

Remark 2.5.15. ([SKK] [63] Chap. II) We have

(1) $\mathcal{E}_{X} \subset \mathcal{E}_{X}^{\infty}$ and $\mathcal{E}_{X} \subset \widehat{\mathcal{E}}_{X}{ }^{6}$

(2) $\mathcal{E}_{X}^{\infty}$ and $\widehat{\mathcal{E}}_{X}$ are faithfully flat ${ }^{7}$ over $\mathcal{E}_{X}$, and $\mathcal{E}_{X}$ is flat over $\pi^{-1} \mathcal{D}_{X}$, where $\pi$ : $T^{*} X \rightarrow X$ is the canonical projection.

(3) The sheaf $\mathcal{E}_{X}, \mathcal{E}_{X}(0)$ and $\widehat{\mathcal{E}}_{X}$ are Noetherian rings on $T^{*} X$.

Proposition 2.5.16. Let $X$ be a complex manifold, then we have

$$
\left.\widehat{\mathcal{E}}_{X}\right|_{T_{X}^{*} X} \cong \mathcal{D}_{X}
$$

\footnotetext{
${ }^{6}$ In [SKK] [63], $\mathcal{E}, \widehat{\mathcal{E}}$ and $\mathcal{E}^{\infty}$ are denoted by $\mathscr{P}^{f}, \widehat{\mathscr{P}}$ and $\mathscr{P}$

${ }^{7} \mathrm{~A}$ module is faithfully flat if taking the tensor product with a sequence produces an exact sequence if and only if the original sequence is exact.
} 


\section{Chapter 3}

\section{Linearization of vector fields}

In section 3.1 we introduce contact vector field and assign each real analytic function a contact vector field. Then we study linearization of vector fields with real analytic coefficients in section 3.2. Later in section 3.3, we discuss eigenvalues of symplectic mappings and clarify the non-resonant condition on the eigenvalues to linearize the contact vector field. At last, Birkhoff normal form is mentioned in section 3.4 .

\subsection{Contact vector fields}

Let $X$ be a $2 n+1$ dimensional manifold, let $L$ be the line subbundle of $T^{*} X$ in section A.2 and let $L^{*}$ be the dual bundle of $L$. Write $\mathcal{A}\left(L^{*}\right)$ as the space of real analytic sections of $L^{*}$. Each $f \in \mathcal{A}\left(L^{*}\right)$ can be regarded as a real analytic function $\tilde{f}$ on $Z=L^{*} \backslash 0$ satisfying

$$
\tilde{f}(x, a w)=a f(x, w)
$$

for $a \in \mathbb{R} \backslash 0$ and $w \in L_{x}$. Conversely, every real analytic function on $Z$, which is homogeneous of degree one in the sense of (3.1), can be identified with a section of $L^{*}$.

Let $Z$ be a $2 n+2$ dimensional symplectic manifold. Functions on $Z$ form a Poisson algebra under the Poisson bracket $\{\cdot, \cdot\}$. There is a Lie algebra homomorphism

$$
\begin{aligned}
\mathcal{A}(Z) & \rightarrow \text { symplectic vector fields } \\
f & \mapsto H_{f}
\end{aligned}
$$

mapping each real analytic function $f$ on $Z$ onto its Hamiltonian vector field $H_{f}$.

Assume $Z$ sits in the symplectic manifold $\left(T^{*} X, \omega\right)$, and the restriction of the symplectic form $\omega$ on $Z$ is also symplectic. The functions of homogeneous of degree one form a Lie subalgebra of the Poisson algebra. Identify these functions as sections of $L^{*}$, then $\mathcal{A}\left(L^{*}\right)$ has a canonical Lie algebra structure with Lie bracket $[\cdot, \cdot]$.

A real analytic function $\tilde{f}$ on $Z$ is homogeneous of degree one, then its Hamiltonian vector field $H_{\tilde{f}}$ is homogeneous of degree zero, and it determines a real analytic vector 
field $V_{f}$ on $Z /(\mathbb{R}-0)=X$. Then $V_{f}$ is a contact vector field and the mapping

$$
\begin{aligned}
\mathcal{A}\left(L^{*}\right) & \rightarrow \text { Contact vector fields with real analytic coefficients } \\
f & \mapsto V_{f}
\end{aligned}
$$

is a Lie algebra homomorphism.

Consider the above discussion in coordinates, take a global section

$$
\alpha=d z+\frac{1}{2} \sum_{i}^{n}\left(x_{i} d y_{i}-y_{i} d x_{i}\right)
$$

of the line bundle $L$ on $\mathbb{R}^{2 n+1}$. Identify $\mathcal{A}\left(\mathbb{R}^{2 n+1}\right)$ with $\mathcal{A}\left(L^{*}\right)$ by $f \rightarrow f \beta$, where $\beta$ is the dual section of the dual bundle $L^{*}$.

Lemma 3.1.1. Let $\left(x_{1}, \cdots, x_{n}, y_{1}, \cdots, y_{n}, z\right)$ be local coordinates of $\mathbb{R}^{2 n+1}$. Given $f \in \mathcal{A}\left(\mathbb{R}^{2 n+1}\right)$, then the associated contact vector field

$$
\begin{aligned}
V_{f}=\sum_{i=1}^{n}\left[\left(\frac{\partial f}{\partial y_{i}}-\right.\right. & \left.\left.\frac{x_{i}}{2} \frac{\partial f}{\partial z}\right) \frac{\partial}{\partial x_{i}}-\left(\frac{\partial f}{\partial x_{i}}+\frac{y_{i}}{2} \frac{\partial f}{\partial z}\right) \frac{\partial}{\partial y_{i}}\right] \\
+ & {\left[\sum_{i=1}^{n}\left(\frac{x_{i}}{2} \frac{\partial f}{\partial x_{i}}+\frac{y_{i}}{2} \frac{\partial f}{\partial y_{i}}\right)-f\right] \frac{\partial}{\partial z} }
\end{aligned}
$$

Proof. Given the one form $\alpha$ defined by (3.2) and introduce $(x, y, z, t)$ as coordinates on $Z=\mathbb{R}^{2 n+2}$, by assigning to $t \alpha$ at $(x, y, z)$ the coordinates $(x, y, z, t)$. Then the symplectic two-form $\Omega$ is

$$
\Omega=d t \wedge \alpha+t d \alpha=d t \wedge\left(d z+\frac{1}{2} \sum_{i}^{n}\left(x_{i} d y_{i}-y_{i} d x_{i}\right)\right)+t \sum d x_{i} \wedge d y_{i}
$$

Given $f \in \mathcal{A}\left(\mathbb{R}^{2 n+1}\right)$, the associated homogeneous function $\tilde{f}$ on $Z$ is just $t f$. Write $H_{\tilde{f}}$ in the form

$$
\sum_{i}\left(a_{i} \partial_{x_{i}}+b_{i} \partial_{y_{i}}+c \partial_{z}+\gamma \partial_{t}\right)
$$

where $a_{i}^{\prime} s, b_{i}^{\prime} s, c$ and $\gamma$ are unknown functions to be determined. We solve the equation

$$
\left.H_{\tilde{f}}\right\lrcorner \Omega=d \tilde{f}
$$

to obtain all the unknown coefficients and we have

$$
\begin{aligned}
H_{\tilde{f}}=\sum_{i=1}^{n} & {\left[\left(\frac{\partial f}{\partial y_{i}}-\frac{x_{i}}{2} \frac{\partial f}{\partial z}\right) \frac{\partial}{\partial x_{i}}-\left(\frac{\partial f}{\partial x_{i}}+\frac{y_{i}}{2} \frac{\partial f}{\partial z}\right) \frac{\partial}{\partial y_{i}}\right] } \\
+ & {\left[\sum_{i=1}^{n}\left(\frac{x_{i}}{2} \frac{\partial f}{\partial x_{i}}+\frac{y_{i}}{2} \frac{\partial f}{\partial y_{i}}\right)-f\right] \frac{\partial}{\partial z}+t \frac{\partial f}{\partial z} \frac{\partial}{\partial t} . }
\end{aligned}
$$

The contact vector field $V_{f}$ can be acquired by projecting $H_{\tilde{f}}$ on $(x, y, z)$ space. 
Corollary 3.1.2. Given $f, g \in \mathcal{A}\left(\mathbb{R}^{2 n+1}\right)$, we define their Lagrange bracket as

$$
\begin{aligned}
\sum_{i=1}^{n}\left(\frac{\partial f}{\partial y_{i}} \frac{\partial g}{\partial x_{i}}-\frac{\partial f}{\partial x_{i}} \frac{\partial g}{\partial y_{i}}\right) & +\left(\sum_{i}^{n}\left(\frac{x_{i}}{2} \frac{\partial f}{\partial x_{i}}+\frac{y_{i}}{2} \frac{\partial f}{\partial y_{i}}-f\right)\right) \frac{\partial g}{\partial z} \\
& -\left(\sum_{i}^{n}\left(\frac{x_{i}}{2} \frac{\partial g}{\partial x_{i}}+\frac{y_{i}}{2} \frac{\partial g}{\partial y_{i}}-g\right)\right) \frac{\partial f}{\partial z}
\end{aligned}
$$

Hint: Let $\tilde{f}=t f$ and $\tilde{g}=t g$ be the homogeneous functions on $Z$ associated with $f$ and $g$. According to Lemma 3.1.1, the Poisson bracket is $H_{\tilde{f}} \tilde{g}$. The Lagrange bracket for two functions in contact space is induced from the formula in Lemma 3.1.1.

\subsection{Linearization of vector fields}

Compare with Guillemin and Schaeffer's methods of linearization in the paper [22], we study linearization of vector fields in real analytic setting instead of smooth setting, i.e., linearize a contact vector field with real analytic coefficients.

In section A.4, we obtain Theorem A.4.21 and Remark A.4.28 on linearizing a real analytic vector field. By applying the techniques of proofs of Poincaré's Theorem A.4.7 and Siegel Theorem A.4.24, we have

Theorem 3.2.1. Let $V$ be a vector field on $\mathbb{R}^{n}$ with real analytic coefficients, and $V$ is of the form

$$
V=V_{0}+V^{\prime}
$$

where $V_{0}$ is linear vector field and $V^{\prime}$ is the collection of nonlinear terms. Suppose the radial point is hyperbolic equilibrium point with all the eigenvalues are distinct and are not equal to zero. Moreover, all the eigenvalues satisfy the conditions in Theorems A.4.21 or Theorem A.4.24 Then there is a germ of analytic homeomorphism,

$$
\varphi:\left(\mathbb{R}^{n}, 0\right) \rightarrow\left(\mathbb{R}^{n}, 0\right)
$$

such that $\varphi_{*} V=V_{0}$.

Consider the contact structure on $\mathbb{R}^{2 n+1}$ defined by the contact form

$$
d z+\frac{1}{2} \sum_{i=1}^{n}\left(x_{i} d y_{i}-y_{i} d x_{i}\right)
$$

from the former discussion, one knows the linear contact vector fields are all of the form

$$
\lambda z \frac{\partial}{\partial z}+\frac{\lambda}{2} \sum\left(x_{i} \frac{\partial}{\partial x_{i}}+y_{i} \frac{\partial}{\partial y_{i}}\right)+\sum\left(\frac{\partial q}{\partial x_{i}} \frac{\partial}{\partial y_{i}}-\frac{\partial q}{\partial y_{i}} \frac{\partial}{\partial x_{i}}\right)
$$

and $q$ is an arbitrary quadratic form in $x$ and $y$. The linear map of $\mathbb{R}^{2 n+1}$ associated with 
it if of the form

$$
\left(\begin{array}{c|c}
\lambda & 0 \cdots 0 \\
\hline 0 & \\
\vdots & \frac{\lambda}{2}+A \\
0 &
\end{array}\right)
$$

and $A \in \operatorname{sp}(2 n)$ is a symplectic matrix. 3.3 is hyperbolic if none of the eigenvalues of $A$ have real part $-\frac{\lambda}{2}$. In this case, the equilibrium point, i.e., the origin, is called hyperbolic equilibrium point. It is well-known that Hartman-Grobman theorem says the behavior of a dynamical system near a hyperbolic equilibrium point is qualitatively the same as the behavior of its linearization near this equilibrium point.

Theorem 3.2.2. (Hartman-Grobman) Let $f: \mathbb{R}^{n} \rightarrow \mathbb{R}^{n}$ be a smooth map of a dynamical system with differential equation $\frac{d u}{d x}=f$. Suppose the map has a hyperbolic equilibrium point $x_{0}$. Then there exists a neighborhood $N$ of the equilibrium $x_{0}$ and a homeomorphism $\varphi: N \rightarrow \mathbb{R}^{n}$, such that $\varphi\left(x_{0}\right)=0$ and such that in the neighborhood $N$, the flow of $\frac{d u}{d x}=f$ is topologically conjugate by the smooth map $v=\varphi(u)$ to the flow of its linearization $\frac{d v}{d x}=A v$.

In general, even for infinitely differentiable maps $f$, the homeomorphism $\varphi$ need not to be smooth, nor even locally Lipschitz. However, it turns out to be Hölder continuous with an exponent depending on the constant of hyperbolicity of $A$.

According to the discussion in $\S 2$ of Guillemin and Schaeffer [22], we have

Theorem 3.2.3. Let $V$ be a real analytic contact vector field on $\mathbb{R}^{2 n+1}$ of the form

$$
V=V_{0}+V^{\prime}
$$

where $V_{0}$ is linear contact vector field and $V^{\prime}$ is the collection of nonlinear terms. Suppose the radial point is hyperbolic equilibrium point with all the eigenvalues are distinct and are not equal to zero. Moreover, all the eigenvalues satisfy the conditions in Theorems A.4.21 or Theorem A.4.24 Then there is a germ of contact transformation,

$$
\varphi:\left(\mathbb{R}^{2 n+1}, 0\right) \rightarrow\left(\mathbb{R}^{2 n+1}, 0\right)
$$

such that $\varphi_{*} V=V_{0}$.

To prove the above theory, we first give two lemmas.

Let $(x, y, z) \in \mathbb{R}^{n} \times \mathbb{R}^{n} \times \mathbb{R}$ be the coordinates of $\mathbb{R}^{2 n+1}$. Consider the polynomial functions on $(x, y) \in \mathbb{R}^{2 n}$ form a subalgebra of the Poisson algebra with respect to

$$
\{f, g\}=H_{f} g=\sum_{i}\left(\frac{\partial f}{\partial y_{i}} \frac{\partial g}{\partial x_{i}}-\frac{\partial f}{\partial x_{i}} \frac{\partial g}{\partial y_{i}}\right) \text {. }
$$

Furthermore, such subalgebra can be set as a graded algebra via decomposition of spaces of homogeneous polynomials. Moreover, a real analytic function can be written as summation of polynomial functions, so any real analytic vector field can be written as a vector field with summations of polynomial functions as its coefficients. The set 
of polynomial functions on $\mathbb{R}^{2 n+1}$, is a Lie subalgebra of $\mathcal{A}\left(\mathbb{R}^{2 n+1}\right)$ with respect to the Lagrange bracket in Corollary 3.1.2. Denote such set by $\mathfrak{g}$, and set $\mathfrak{g}^{i}$ to be the sum of all polynomials $z^{l} h(x, y)$, where $h(x, y)$ is a homogeneous polynomial in $(x, y)$ of degree $k$. Moreover we have $2 l+k-2=i$. Then $\mathfrak{g}=\oplus_{i=-2}^{\infty} \mathfrak{g}^{i}$ is a graded Lie algebra sum and $\left[\mathfrak{g}^{i}, \mathfrak{g}^{j}\right] \subset \mathfrak{g}^{i+j}$ (notice that $\operatorname{ad}_{z}\left(\mathfrak{g}^{i}\right)=\left[z, \mathfrak{g}^{i}\right]=-\frac{i}{2} \mathfrak{g}^{i}$, and use the Jacobi's identity).

However, the graded Poisson algebra in $\mathbb{R}^{2 n}$ can be recognized as the subalgebra of polynomial functions in $(x, y)$, which is contained in the graded Lie algebra $\mathfrak{g}$. Moreover $\mathfrak{g}^{0}=\operatorname{sp}(2 n)+\{c z\}$. For $G \in \mathfrak{g}^{0}$, consider the centeralizer set

$$
C(G)=\{m \mid m \in \mathfrak{g},[m, G]=0\} .
$$

Set the collection $\mathfrak{G}$ of all elements $G$ of $\mathfrak{g}^{0}$ satisfy $C(G) \subset \mathfrak{g}^{0}$. Notice that $\mathfrak{G}$ is of second category. Apply the same discussion to the ring of formal power series in $x, y$ and $z$. The Lagrange bracket in Corollary 3.1.2 also defines a Lie algebra $\overline{\mathfrak{g}}$ and

$$
\overline{\mathfrak{g}}=\oplus_{i=-2}^{\infty} \mathfrak{g}^{i} .
$$

In additional, there is a subalgebra $h$ of $\overline{\mathfrak{g}}$, is the infinite sum $h=\oplus_{i=0}^{\infty} \mathfrak{g}^{i}$.

Given $V \in h$, we have the formal sum

$$
\exp (\operatorname{ad} V)=I+\operatorname{ad} V+\frac{1}{2}(\operatorname{ad} V)^{2}+\frac{1}{3 !}(\operatorname{ad} V)^{3}+\cdots,
$$

which is converges in the sense of formal power series. $\exp (V)$ gives an automorphism of $\mathfrak{\mathfrak { g }}$. Similar as the Theorem 1 in $\S 2$ of [22], we have

Lemma 3.2.4. Let $V=\sum_{i=0}^{\infty} V_{i}$ be in $h$. Assume $V_{0} \in \mathfrak{g}^{0}$ of $\overline{\mathfrak{g}}$ satisfies $V_{0} \in \mathfrak{G}$. Moreover, all the eigenvalues associated with $V_{0}$ satisfy the conditions in Theorems A.4.13 Then there is an formal automorphism $\varphi$ of $\overline{\mathfrak{g}}$ such that $\varphi V=V_{0}$.

Proof. With the above discussion, the bijective mapping ad $V_{0}: \mathfrak{g}^{1} \rightarrow \mathfrak{g}^{1}$ implies the existence of $V_{1}^{\prime} \in \mathfrak{g}^{1}$ such that $\left[V_{1}^{\prime}, V_{0}\right]=V_{1}$. Let $\varphi_{1}=\exp \left(\operatorname{ad} V_{1}^{\prime}\right)$, then $\varphi_{1}(V)=V_{0}+$ $W_{2}$ where $W_{2} \in \oplus_{i=2}^{\infty} \mathfrak{g}^{i}$. Write $W_{2}=V_{2}+W_{3}$, where $V_{2}$ is the leading term of $W_{2}$ and $W_{3} \in \oplus_{i=3}^{\infty} \mathfrak{g}^{i}$, the bijective mapping ad $V_{1}: \mathfrak{g}^{2} \rightarrow \mathfrak{g}^{2}$ implies the existence of $V_{2}^{\prime} \in \mathfrak{g}^{2}$ such that $\left[V_{2}^{\prime}, V_{1}\right]=V_{2}$. Let $\varphi_{2}=\exp \left(\operatorname{ad} V_{2}^{\prime}\right)$, then $\varphi_{2} \circ \varphi_{1}(V)=V_{0}+W_{3}$. Repeat the step and each time we eliminate the leading nonlinear term, then we formulate a formal mapping

$$
\varphi=\lim _{i \rightarrow \infty} \varphi_{i} \circ \varphi_{i-1} \circ \cdots \circ \varphi_{1}
$$

linearize the vector $V$.

Consider any $2 n+1$ dimensional contact manifold $(X, L)$, and let $V$ be a contact vector field on $X$ with $x_{0}$ be its zero. The linear part of any real analytic vector field gives a $(2 n+1) \times(2 n+1)$ matrix 3.3 . Treat $\frac{\lambda}{2}+A$ as the element of $\lambda z+A \in \mathfrak{g}^{0}$, then we have

Lemma 3.2.5. If $\lambda z+A$ satisfies that the center $C(A)$ of $A$ is contained in $\mathfrak{g}^{0} \bigsqcup^{1}$ then

\footnotetext{
${ }^{1}$ The non-resonance condition in section 4.2 insure this condition is fulfilled.
} 
there is a germ of formal contact transformation $\varphi:\left(X, x_{0}\right) \rightarrow\left(X, x_{0}\right)$ such that

$$
\varphi_{*} V=V_{0}
$$

where $V_{0}$ is the linear contact vector field on $X$ with $\lambda z+A \in \mathfrak{g}^{0}$.

Now we are going to finish the proof of Theorem 3.2 .3 .

Proof. With the above Lemma 3.2.4 and Lemma 3.2, we already give a formal contact transformation $\varphi$ such that the push forward of $\varphi$ linearizes the contact vector field $V$. Now we want to prove the formal mapping $\varphi$ is convergent to a real analytic mapping.

However, in Appendix A.4, we have show the convergence of the formal mapping with some conditions of the eigenvalues of $A$. Write $\left\{ \pm \lambda_{i}, i=1, \cdots, n\right\}$ as the $2 n$ eigenvalues of $A$. Consider the matrix (4.14) induced from the normal form 2 of operators in our problem. On one hand, consider the attracting/repelling cases of our operators, the $2 n+1$ eigenvalues

$$
\left\{\gamma, \frac{\gamma}{2} \pm \lambda_{1}, \cdots, \frac{\gamma}{2} \pm \lambda_{n}\right\}
$$

of the matrix (3.3) are of of Poincare type 3, which is fulfill the conditions of Theorem A.4.21. We use majorant series and construct contract mapping to prove the convergence, see the proof of Theorem A.4.21 for the construction.

On the other hand, for the non-attracting/non-repelling cases, the $2 n+1$ eigenvalues $\left\{\gamma, \frac{\gamma}{2} \pm \lambda_{1}, \cdots, \frac{\gamma}{2} \pm \lambda_{n}\right\}$ are of Siegel type with additional condition such as some Diophantine condition in Definition A.4.23. Such additional condition insure that the inverse operator $\mathrm{ad}_{\Lambda}^{-1} 4^{4}$ will not vanish to fast. Then by applying the Theorem A.4.24, one can finish the proof by using corresponding technique from the classical KAM theory, whose name is after A. Kolmogorov, V. Arnold and J. Moser, to deal with the small denominator that does not vanish too fast. See Chapter 2 of [9] or Chapter I, section $5 \mathrm{E}$ of [32] for introductions.

Remark 3.2.6. In Theorem 2 of [22], they obtained a contact transformation push forward the contact vector field $V$ to a linear vector field $V_{0}$ plus a term $V_{\infty}$ vanishes to infinite order at equilibrium point. However, in real analytic setting, we do not have the latter term due to the property of real analytic coefficients. That is why Guillemin and Schaeffer spent one section to cancel the term $V_{\infty}$ but we only need to prove the convergence of $\varphi$.

Remark 3.2.7. For the operators in our problem in section 1.3 , we only need eigenvalues of $A$ satisfy the non-resonant condition 5 and the eigenvalues are of Poincaré type or to be Liouvillean (Siegel type satisfying some Diophantine condition). However, The

\footnotetext{
${ }^{2}$ See section 4.3 for details.

3 See Definition A.4.5 Roughly, the convex hull of all eigenvalues does not contain the origin. Otherwise, the eigenvalues are of Siegel type.

${ }^{4}$ See Definition A.4.11 for the definition of the operator $\operatorname{ad}{ }_{\Lambda}$.

${ }^{5}$ See section 4.2
} 
latter one is not rigorous for our proof of the above lemma. For eigenvalues of Siegel type, there are more cases available for our linearization theory, see Appendix A.4.

\subsection{Eigenvalues of symplectic mappings}

Let $E$ be a 2 n dimensional vector space equipped with a linear symplectic form $\Omega$. Denote by $\operatorname{sp}(E)$ the set of linear mappings $A: E \rightarrow E$ such that

$$
\Omega(A v, w)+\Omega(v, A w)=0
$$

for all $v, w \in E$ and by $\operatorname{Sp}(E)$ the group of linear mappings $B: E \rightarrow B$ such that

$$
\Omega(B v, B w)=\Omega(v, w) .
$$

Henceforth $\operatorname{Sp}(E)$ is the symplectic group and $\operatorname{sp}(E)$ its Lie algebra.

We only consider $A \in \operatorname{sp}(E)$ is semi-simple, that is, diagnosable, and the eigenvalues of $A$ are distinct. The eigenvalues of $A$ come in groups of three types:

(i) pairs $\lambda,-\lambda$ of inverse real eigenvalues,

(ii) pairs $\lambda, \bar{\lambda}$ of conjugate eigenvalues which are pure imaginary,

(iii) four tuplets $\lambda, \bar{\lambda},-\lambda,-\bar{\lambda}$ of complex eigenvalues but not pure imaginary.

From the knowledge of symplectic geometry, we have

Lemma 3.3.1. The symplectic form $\Omega$ restricted to each non-decomposable subspace $E^{\lambda}$ is non-degenerate. Furthermore, all the $E^{\lambda}$ 's are mutually perpendicular with respect to $\Omega$.

Definition 3.3.2. Say $A$ is elliptic if all its eigenvalues are of type (ii), hyperbolic if they are all of type (i), and loxodromic if they all come in quadruples (iii). Otherwise, we call $A$ is of mixed type.

Theorem 3.3.3. [22] If $A \in \operatorname{sp}(E)$ is hyperbolic or loxodromic, then it is conjugate to an element of $\operatorname{sp}(2 n)$ of the form

$$
\left(\begin{array}{c|c}
B & 0 \\
\hline 0 & B
\end{array}\right) .
$$

If $A$ is elliptic, it is conjugate to an element of the form

$$
\left(\begin{array}{c|c}
0 & -D_{1} \\
\hline D_{2} & 0
\end{array}\right)
$$

where $D_{1}$ and $D_{2}$ are diagonal matrices. Moreover we can arrange that the diagonal entries of $D_{1}$ and $\pm 1^{\prime} s$, and that the $i$-th diagonal entries of $D_{1}$ and $D_{2}$ have the same sign. 
Equip $\mathbb{R}^{2 n+1}$ with contact form $d z+\frac{1}{2} \sum\left(x_{i} d y_{i}-y_{i} d x_{i}\right)$, and let $V$ be a linear contact vector field of the form

$$
\lambda z \frac{\partial}{\partial z}+\frac{\lambda}{2} \sum\left(x_{i} \frac{\partial}{\partial x_{i}}+y_{i} \frac{\partial}{\partial y_{i}}\right)+\sum\left(\frac{\partial q}{\partial x_{i}} \frac{\partial}{\partial y_{i}}-\frac{\partial q}{\partial y_{i}} \frac{\partial}{\partial x_{i}}\right),
$$

where $q$ is an arbitrary quadratic form in $x$ and $y$. Split $V$ into two parts as following,

$$
\begin{gathered}
V_{2}=\lambda z \frac{\partial}{\partial z}+\frac{\lambda}{2} \sum\left(x_{i} \frac{\partial}{\partial x_{i}}+y_{i} \frac{\partial}{\partial y_{i}}\right) \\
V_{1}=\sum\left(\frac{\partial q}{\partial x_{i}} \frac{\partial}{\partial y_{i}}-\frac{\partial q}{\partial y_{i}} \frac{\partial}{\partial x_{i}}\right)
\end{gathered}
$$

Set $S^{k}$ be the space of homogenous polynomials of degree $k$ in $x$ and $y$, and set

$$
S^{k, l}=\left\{z^{l} h, h \in S^{k}\right\} .
$$

Denote an element of $\operatorname{Aut}\left(S^{k}\right)$ by

$$
V_{1}=V_{1}^{(k)}: S^{k} \rightarrow S^{k}
$$

One also has $V_{1}^{(k)} \in \operatorname{Aut}\left(S^{k, l}\right)$ via $S^{k} \cong S^{k, l}$ given by $h \rightarrow z^{l} h$.

The vector field $V$ acts on $S^{k, l}$ in two ways.

(a) $V$ acts on $S^{k, l}$ by means of the Lagrange bracket as:

$$
\left(\frac{k}{2}+l-1\right) \lambda I+V_{1}^{(k)}
$$

With respect to Lagrange bracket, the first part $V_{2}$ of $V$ acts as $\left(\frac{k}{2}+l-1\right) \lambda I$, and the second part $V_{1}$ act as $V_{1}^{(k)}$.

(b) $V$ act as a linear mapping on $S^{k, l}$ by the rule for differentiating a homogeneous function by a vector field:

$$
\left(\frac{k}{2}+l\right) \lambda I+V_{1}^{(k)}
$$

We have the following generical conditions of eigenvalues:

(i) First to linearize an arbitrary formal contact vector field with linear part $V$, one need (a) to be non-singular for $\left(\frac{k}{2}+l-1\right)>0$ (see Remark 3.2.6, and see [22], $\S 2$ for details), that is $-\frac{m \lambda}{2}$ is not an eigenvalue of $V_{1}^{(k)}$ for $m>0$.

(ii) We will see in step (II) of the proof of Corollary 4.3.2, to obtain the normal form, one has formula (3.5) in (b), we need

$-\frac{m^{\prime} \lambda}{2}$ is not an eigenvalue of $V_{1}^{(k)}$ for $m^{\prime}>0$. 
(iii) Remember for the linearization we need the zero of the vector field is hyperbolic, that is the matrix $\frac{\lambda I}{2}+A$ has no imaginary eigenvalues.

Applying the above conditions we can define a generical radial point ${ }^{6}$

\subsection{Birkhoff normal form}

Next chapter we will give the normal form of our operators in section 1.3, it is worth to mention some knowledge of Birkhoff normal form, the reference is [1].

Write the hamiltonian as $H=\frac{1}{2}(A x, x)$, where $x=\left(p_{1}, \cdots, p_{n} ; q_{1}, \cdots, q_{n}\right)$ is a vector written in a symplectic basis and $A$ is a symmetric linear operator. The canonical equations have the form

$$
\dot{x}=I A x, \quad I=\left(\begin{array}{cc}
0 & -E \\
E & 0
\end{array}\right) .
$$

By eigenvalues of the hamiltonian we mean the eigenvalues of the linear infinitesimally symplectic operator $I A$, and by a Jordan block we mean a Jordan block of the operator $I A$.

The eigenvalues of the hamiltonian are of four types: real pairs $(a,-a)$, purely imaginary pairs $(\sqrt{-1} b,-\sqrt{-1} b)$, quadruples $( \pm a \pm \sqrt{-1} b)$ and zero eigenvalues. The Jordan blocks corresponding to the two members of a pair or four members of a quadruple always have the same structure.

The complete list of normal forms follows:

(1) For a pair of Jordan blocks of order $k$ with eigenvalues $\pm a$, then hamiltonian is

$$
H=-a \sum_{j=1}^{k} p_{j} q_{j}+\sum_{j=1}^{k-1} p_{j} q_{j+1}
$$

(2) For a quadruple of Jordan blocks of order $k$ with eigenvalues $\pm a \pm \sqrt{-1} b$ the hamiltonian is

$$
H=-a \sum_{j=1}^{2 k} p_{j} q_{j}+b-a \sum_{j=1}^{k}\left(p_{2 j-1} q_{2 j}-p_{2 j} q_{2 j-1}\right)+\sum_{j=1}^{2 k-2} p_{j} q_{j+2}
$$

(3) For a pair of Jordan blocks of order $k$ with eigenvalues zero the hamiltonian is

$$
H=\sum_{j=1}^{k-1} p_{j} q_{j+1} \quad(\text { for } \quad k=1, H=0) .
$$

\footnotetext{
${ }^{6}$ See Definition 4.2 .4
} 
(4) For a Jordan blocks of order $2 k$ with eigenvalues zero the hamiltonian is of one of the following two inequivalent types:

$$
H= \pm \frac{1}{2}\left(\sum_{j=1}^{k-1} p_{j} q_{k-j}-\sum_{j=1}^{k} q_{j} q_{k-j-1}\right)-\sum_{j=1}^{k-1} p_{j} q_{j+1}
$$

for $k=1$, this is $H= \pm \frac{1}{2} q_{1}^{2}$.

(5) For a pair of Jordan blocks of order $2 k+1$ with purely imaginary eigenvalues $\pm \sqrt{-1} b$ the hamiltonian is of one of the following two inequivalent types:

$$
\begin{aligned}
H= & \pm \frac{1}{2}\left[\sum_{j=1}^{k}\left(b^{2} p_{2 j} q_{2 k-2 j+2}+q_{2 j} q_{2 k-2 j+2}\right)\right. \\
& \left.-\sum_{j=1}^{k+1}\left(b^{2} p_{2 j-1} q_{2 k-2 j+3}+q_{2 j-1} q_{2 k-2 j+3}\right)\right]-\sum_{j=1}^{2 k} p_{j} q_{j+1},
\end{aligned}
$$

for $k=0, H= \pm \frac{1}{2}\left(b^{2} p_{1}^{2}+q_{1}^{2}\right)$.

(6) For a pair of Jordan blocks of order $2 k$ with eigenvalues $\pm \sqrt{-1} b$ the hamiltonian is of one of the following two inequivalent types:

$$
\begin{aligned}
H= & \pm \frac{1}{2}\left[\sum_{j=1}^{k}\left(\frac{1}{b^{2}} q_{2 j-1} q_{2 k-2 j+1}+q_{2 j} q_{2 k-2 j+2}\right)\right. \\
& \left.-\sum_{j=1}^{k-1}\left(b^{2} p_{2 j+1} q_{2 k-2 j+1}+p_{2 j+2} q_{2 k-2 j+2}\right)\right]-b^{2} \sum_{j=1}^{2 k} p_{2 j-1} q_{2 j}+\sum_{j=1}^{2 k} p_{2 j} q_{2 j-1},
\end{aligned}
$$

for $k=1, H= \pm \frac{1}{2}\left(\frac{1}{b^{2}} q_{1}^{2}+q_{2}^{2}\right)-b^{2} p_{1} q_{2}+p_{2} q_{1}$.

Theorem 3.4.1. (Williamson's theorem) A real symplectic vector space with a given quadratic form $H$ can be decomposed into a direct sum of pairwise skew orthogonal real symplectic subspaces so that the form $H$ is represented as a sum of forms of the types indicated above on these subspaces.

For our case, we only need to consider all the Jordan blocks are of first order, while the individual hamiltonian in "general position" does not have multiple eigenvalues and reduces to a simple form. Moreover, in our case, we consider the principal symbol as the Hamiltonian of quadratic form in the variable $\left(x^{\prime}, \xi^{\prime}\right)$. 


\section{Chapter 4}

\section{The normal form}

In this chapter we are going to simplify the microdifferential operator $P$ near a generic radial point. Basically first we introduce the subprincipal symbol in section 4.1, after that we study analytic properties of radial points and clarify the generic conditions in section 4.2, then obtain the normal form of the original operator $P$ in section 4.3 . We discuss the projected null bicharacteristics at the end this chapter.

\subsection{Subprincipal symbol}

Let $X$ be an $n$ dimensional real analytic manifold, let $P$ be a microdifferential operator of order $m$ defined on $S^{*} X$, and Take a local coordinate system $x=\left(x_{1}, x_{2}, \cdots, x_{n}\right)$ of $X$. By considering the adjoint operator $P^{*}$ of $P=\left\{P_{k}(x, \xi)\right\}_{k}$, we have

$$
\begin{gathered}
\left(P^{*}\right)_{m}(x, \xi)=P_{m}(x,-\xi)=(-1)^{m} P_{m}(x, \xi) \\
\left(P^{*}\right)_{m-1}(x, \xi)=P_{m-1}(x,-\xi)-\sum_{j} \frac{\partial^{2}}{\partial x_{j} \partial \xi_{j}} P_{m}(x,-\xi) \\
=(-1)^{m}\left(P_{m-1}(x, \xi)-\sum_{j} \frac{\partial^{2}}{\partial x_{j} \partial \xi_{j}} P_{m}(x, \xi)\right) .
\end{gathered}
$$

Hence $P-(-1)^{m} P^{*} \in \mathcal{E}_{X}(m-1)$.

Definition 4.1.1. We set

$$
\begin{aligned}
\sigma_{\mathrm{sub}}^{m-1}(P) & :=\frac{1}{2} \sigma_{m-1}\left(P-(-1)^{m} P^{*}\right) \\
& =P_{m-1}-\frac{1}{2} \sum_{j} \frac{\partial^{2} P_{m}}{\partial x_{j} \partial \xi_{j}}
\end{aligned}
$$

and call it the subprincipal symbol $\mathrm{1}^{1}$ of $P$.

\footnotetext{
${ }^{1}$ Notice that if we write a pseudo-differential operator $P(x, D)=P_{0}(x)+\sum_{i=1}^{m} P_{i}(x, D)$ on $\mathbb{R}^{n}$ with $D=\frac{1}{\sqrt{-1}} \frac{\partial}{\partial x}$, the formula of its subprincipal symbol is like $\sigma_{\mathrm{sub}}^{m-1}(P):=P_{m-1}-\frac{1}{2 \sqrt{-1}} \sum_{j} \frac{\partial^{2} P_{m}}{\partial x_{j} \partial \xi_{j}}$.
} 
The map $\sigma_{\text {sub }}^{m-1}: \mathcal{E}_{X}(m) \rightarrow \mathscr{O}_{T^{*} X}$ extends the principal symbol $\sigma_{m-1}: \mathcal{E}_{X}(m-$ $1) \rightarrow \mathscr{O}_{T^{*} X}$. However, unlike the principal symbol, it depends on the choices of coordinate systems. Suppose $\tilde{P}=\left(\tilde{P}_{k}(\tilde{x}, \tilde{\xi})\right)$ be the associated operator of $P$ in another coordinated system $\tilde{x}=\left(\tilde{x}_{1}, \cdots, \tilde{x}_{n}\right)$. One has $d \tilde{x} \tilde{P}^{*} d \tilde{x}^{-1}=d x P^{*} d x^{-1}$, and let $\tilde{\sigma}_{m-1}(\tilde{P})$ be the subprincipal symbol in $\tilde{x}$, one has

$$
\tilde{\sigma}_{\text {sub }}^{m-1}(\tilde{P})=\sigma_{\text {sub }}^{m-1}(P)-\frac{1}{2}\left\{\sigma_{m}(P), \log \frac{d(\tilde{x})}{d x}\right\} .
$$

Then one can define a first order differential operator $\mathscr{L}_{P}^{(m-1)}$ acting on $\mathscr{O}_{T^{*} X} \otimes \Omega_{X}^{-1 / 2}$ by

$$
\mathscr{L}_{P}^{(m-1)}(a / \sqrt{d x}):=\left(H_{\sigma_{m}(P)} a+\sigma_{\text {sub }}^{m-1}(P) a\right) / \sqrt{d x},
$$

where $\Omega_{X}^{-1 / 2}=\left(\Omega_{X}^{1 / 2}\right)^{\otimes-1}$, and $\Omega_{X}^{1 / 2}$ is an invertible sheaf such that $\left(\Omega_{X}^{1 / 2}\right)^{\otimes 2}=\Omega_{X}$, and let $\sqrt{d x}$ denote a section of $\Omega_{X}^{1 / 2}$ such that $(\sqrt{d x})^{\otimes 2}=d x$, here $d x=d x_{1} \wedge \cdots \wedge$ $d x_{n} \in \Omega_{X}$. By definition, $(d x)^{\frac{1}{2}} \mathscr{L}_{P}^{(m-1)}(d x)^{\frac{1}{2}}$ is independent of the choice of local coordinated system.

Proposition 4.1.2. For $P \in \mathcal{E}_{X}(m)$ and $Q \in \mathcal{E}_{X}(n)$, one has

(1) $\sigma_{\mathrm{sub}}^{m+n-1}(P Q)=\sigma_{m}(P) \sigma_{\mathrm{sub}}^{n-1}(Q)+\sigma_{\mathrm{sub}}^{m-1}(P) \sigma_{n}(Q)+\frac{1}{2}\left\{\sigma_{m}(P), \sigma_{n}(Q)\right\}$,

(2) $\sigma_{\mathrm{sub}}^{m+n-2}([P, Q])=\left\{\sigma_{m}(P), \sigma_{\mathrm{sub}}^{n-1}(Q)\right\}+\left\{\sigma_{\text {sub }}^{m-1}(P), \sigma_{n}(Q)\right\}$,

(3) $\mathscr{L}_{P Q}^{m+n-1}=P_{m} L_{Q}^{(n-1)}+Q_{n} \mathscr{L}_{P}^{(m-1)}+\frac{1}{2}\left[P_{m}, Q_{n}\right]$,

(4) $\mathscr{L}_{[P, Q]}^{m+n-2}=\left[\mathscr{L}_{P}^{(m-1)}, \mathscr{L}_{Q}^{(n-1)}\right]$.

\subsection{Classification of radial points}

Let $X$ be an $n$-dimensional manifold and let $(x, \xi)=\left(x_{1}, \cdots, x_{n}, \xi_{1}, \cdots, \xi_{n}\right)$ be local coordinates of $T^{*} X$. Let $P$ be an $m$-th order microdifferential operator defined on $T^{*} X$ with principal symbol $p_{m}(x, \xi)$, the characteristic variety $\operatorname{Char}(P)$ of the operator $P$ is defined by

$$
\operatorname{Char}(P):=\left\{(x, \xi) \in T^{*} X \backslash 0 \mid p_{m}(x, \xi)=0\right\},
$$

which is a closed subset of $T^{*} X$.

Definition 4.2.1. A point $\nu_{0}=\left(x_{0}, \xi_{0}\right)$ in Char $(P)$ is said to be a radial point of the operator $P$ if the Hamiltonian vector field $H_{p_{m}}$ associated with the principal symbol $p_{m}$ of $P$ is a (necessarily nonzero) multiple of the radial vector field $R=\xi \frac{\partial}{\partial \xi}$, i.e., $H_{p_{m}}+\gamma R=0$ holds at $\nu_{0}$ for some $\gamma \in \mathbb{R} \backslash 0$. Conversely, if $H_{p_{m}}$ and $R$ are linear independent at $\nu^{0}$, then we say the operator $P$ is of principal type at $\nu^{0}$.

Remark 4.2.2. There is an equivalent definition of radial point that $d p_{m}$ and the canonical 1-form $\alpha=\xi d x$ are collinear at $\left(x_{0}, \xi_{0}\right)$. A point $\left(x_{0}, \xi_{0}\right)$ is radial if and 
only if it solves the system

$$
\begin{cases}\frac{\partial p_{m}}{\partial \xi_{i}}=0, & i=1, \cdots, n, \\ \xi_{n} \frac{\partial p_{m}}{\partial x_{i}}=\xi_{i} \frac{\partial p_{m}}{\partial x_{n}}, & i=1, \cdots, n-1 .\end{cases}
$$

If $\left(x_{0}, \xi_{0}\right)$ is a radial point, then all points $\left(t x_{0}, t \xi_{0}\right)(t \neq 0)$ in the direction are radial, eventually we can consider problem on cotangent sphere bundle $S^{*} X$. Here we prefer to consider $S^{*} X$ instead of $P^{*} X$, because it is more convenient to study the singular spectrum.

Example 4.2.3. Consider a simple example of Euler operator

$$
P=x D_{x}-\theta, \quad x \in \mathbb{R}
$$

where $\theta \in \mathbb{C}$. It has two radial points $(0, \pm 1)$ on $S^{*} \mathbb{R}$, and its subprincipal symbol is $\frac{\sqrt{-1}}{2}-\theta$.

A radial point $\nu_{0}$ is isolated in microlocal sense if there is no other ray near $\mathbb{R}_{+} \nu_{0}$ consisting of radial points.

We will see later in next section that linearizing the contact vector field $H_{p_{m}}+\gamma R$ gives a matrix $\frac{\gamma}{2} I+A$, where $A$ is a symplectic matrix. According to section 3.3 . suppose the eigenvalues of the symplectic mapping $A$ at the radial point $\left(x_{0}, \xi_{0}\right)$ are distinct, and we can write them as

$$
\lambda_{1},-\lambda_{1}, \lambda_{2},-\lambda_{2}, \cdots, \lambda_{n},-\lambda_{n}
$$

Definition 4.2.4. (Non-resonant condition) A radial point $\left(x_{0}, \xi_{0}\right)$ is generic if the equation

$$
m \frac{\gamma}{2}=\sum_{i=1}^{n} m_{i} \operatorname{R} e \lambda_{i}
$$

has no integer solution $\left(m, m_{1}, \cdots, m_{n}\right) \in \mathbb{Z}^{n+1}$ with $m \neq 0$.

Definition 4.2.5. A generic radial point $\nu_{0}$ is called to be elliptic (hyperbolic, loxodromic or of mixed type, respectively) if the associated symplectic matrix $A$ is elliptic (hyperbolic, loxodromic or of mixed type, respectively).

Near a generic radial point, the microlocal equivalence of operators is classified by three invariants [75]:

(i) The factor $\gamma \in \mathbb{R} \backslash 0$, with $H_{p_{m}}+\gamma R=0$ at the radial point $\nu_{0}$. One can see $\gamma$ is homogeneous in $\xi_{0}$ of order $m-1$, i.e.,

$$
\gamma_{P}^{m-1}\left(t \nu_{0}\right)=t^{m-1} \gamma_{P}^{m-1}\left(\nu_{0}\right)
$$

here we write $\gamma$ as $\gamma_{P}^{m-1}\left(\nu_{0}\right)$. 
(ii) The subprincipal symbol of $P$ at $\left(x_{0}, \xi_{0}\right)$

$$
\sigma_{\text {sub }}^{m-1}(P)\left(x_{0}, \xi_{0}\right)=p_{m-1}\left(x_{0}, \xi_{0}\right)-\frac{1}{2 i} \sum_{j=1}^{n} \frac{\partial^{2} p_{m}}{\partial x_{j} \partial \xi_{j}}\left(x_{0}, \xi_{0}\right) .
$$

The subprincipal symbol is an invariant in the sense as following [75]:

Lemma 4.2.6. Let $P$ be an $m$-th order pseudo-differential operator and $\nu_{0}$ be a radial point, and let $U$ be an open neighborhood of $\nu_{0}$ in $T^{*} X \backslash 0$. Let $J$ be an elliptic Fourier integral operator with associated canonical transformation $\mathscr{J}: U \rightarrow \mathscr{J}(U)$. Then

$$
\left.\sigma_{\text {sub }}^{m-1}\left(J P J^{-1}\right)\left(\mathscr{J}\left(\nu_{0}\right)\right)=\sigma_{\text {sub }}^{m-1}(P)\left(\nu_{0}\right)\right)
$$

and $J^{-1}$ is the parametrix of $J$.

(iii) Conformal symplectic map $\frac{\gamma}{2} I+A$.

From the definition, at radial point $\left(x_{0}, \xi_{0}\right)$ we have

$$
\left.\left(H_{p}+\gamma R\right)\right|_{\left(x_{0}, \xi_{0}\right)}=0,
$$

where $H_{p}$ is symplectic vector field and $\gamma R$ is conformal symplectic vector field with constant conformal factor $u$. Extend $H_{p}+\gamma R$ to a vector field $H_{p_{m}}+\varphi(x, \xi) R$ with $\left(x_{0}, \xi_{0}\right)$ as its zero. Here $\varphi: T^{*} X \backslash 0 \rightarrow \mathbb{R}$ is arbitrary real function homogeneous of degree $m-1$ with value $\gamma$ at $\left(x_{0}, \xi_{0}\right)$. Let $V_{P}^{0}$ be the linear part of the vector field at $\left(x_{0}, \xi_{0}\right)$, then it defines a conformal symplectic linear map

$$
V_{P}^{0}: T_{\left(x_{0}, \xi_{0}\right)}\left(T^{*} X \backslash 0\right) \rightarrow T_{\left(x_{0}, \xi_{0}\right)}\left(T^{*} X \backslash 0\right)
$$

of the form

$$
\left(\begin{array}{c|c}
\varphi & 0 \cdots 0 \\
\hline 0 & \\
\vdots & \frac{\varphi}{2}+A \\
0 &
\end{array}\right)
$$

where $A$ is symplectic. Also we have tensor contraction $\left\langle R, d p_{m}\right\rangle=k d p_{m}$ and $\left\langle H_{p_{m}}, d p_{m}\right\rangle=0$, which imply that the transpose of $V_{P}^{0}$

$$
\left(V_{p}^{0}\right)^{t}: T_{\left(x_{0}, \xi_{0}\right)}^{*}\left(T^{*} X \backslash 0\right) \rightarrow T_{\left(x_{0}, \xi_{0}\right)}^{*}\left(T^{*} X \backslash 0\right)
$$

maps $d p_{m}$ onto a multiple of itself, and alternatively, $V_{P}^{0}$ maps the codimension one subspace of $T_{\nu_{0}}\left(T^{*} X \backslash 0\right)$ defined by $d p_{m}\left(\nu_{0}\right)=0$ into itself.

Moreover, it introduces two invariant spaces, one is spanned by $H_{p_{m}}\left(x_{0}, \xi_{0}\right)$ (or $\left.R\right|_{\left(x_{0}, \xi_{0}\right)}$ ), another is the kernel of $d p_{m}(x, \xi)$ (or $\alpha$ ) quotient the subspace spanned by $H_{p_{m}}(x, \xi)$ (or $R$ ), i.e., $\widehat{E}_{\hat{\nu}_{0}}:=\alpha^{\perp} / \mathbb{R}_{+} R$, where $\hat{\nu}_{0}$ is image of $\nu_{0}$ under the canonical projection $T^{*} X \backslash 0 \rightarrow S^{*} X$. That is $V_{P}^{0}$ induces a symplectic map $V_{P}$ of the form $\frac{\varphi}{2} I+A$ on $\widehat{E}_{\hat{\nu}_{0}}$, which is also conformal, and is independent on the choice of $\varphi$, where $A \in \operatorname{sp}\left(\widehat{E}_{\hat{\nu}_{0}}\right)$. We have

$$
\frac{\gamma}{2} I+A\left(\nu_{0}\right) \in \operatorname{csp}\left(\widehat{E}_{\hat{\nu}_{0}}\right)
$$


Since $A$ is a $(n-1) \times(n-1)$ matrix associated with the operator $P$, we write $A=A_{P}^{m-1}$.

\subsection{Normal form}

Similar as Witt [75], we have

Theorem 4.3.1. Let $\mathscr{M}$ and $\mathscr{M}^{\prime}$ are two $\mathcal{E}_{X}$-modules associated with two $m$-th order micro-differential operators $P$ and $P^{\prime}$, respectively, and let $\nu_{0}$ and $\nu_{0}^{\prime}$ be radial points of $P$ and $P^{\prime}$, respectively. If $\mathscr{M}$ near $\nu_{0}$ and $\mathscr{M}^{\prime}$ near $\nu_{0}^{\prime}$ are microlocally equivalent, via quantized contact transformation, then one has three invariants:

(i) $\gamma_{P}^{m-1}\left(\nu_{0}\right)=\gamma_{P^{\prime}}^{m-1}\left(\nu_{0}^{\prime}\right)$,

(ii) $\left.\sigma_{\text {sub }}^{(m-1)}(P)\right|_{\nu_{0}}=\left.\sigma_{\text {sub }}^{(m-1)}\left(P^{\prime}\right)\right|_{\nu_{0}^{\prime}}$,

(iii) There is a linear symplectic map $W: \widehat{E}_{\hat{\nu}_{0}} \rightarrow \widehat{E}_{\hat{\nu}_{0}^{\prime}}$ such that

$$
A_{P^{\prime}}^{m-1}\left(\nu_{0}^{\prime}\right)=W A_{P}^{m-1}\left(\nu_{0}\right) W^{-1} .
$$

If $\nu_{0}$ and $\nu_{0}^{\prime}$ are generic, then these conditions are also sufficient, that is, the three invariants imply that $\mathscr{M}$ and $\mathscr{M}^{\prime}$ are microlocally equivalent.

Proof. Suppose that two $\mathcal{E}_{X}$-modules $\mathscr{M}$ near $\nu_{0}$ and $\mathscr{M}^{\prime}$ near $\nu_{0}^{\prime}$ are microlocally equivalent. That is, $P^{\prime}$ is equivalent to $P$ via conjugating invertible microdifferential operator of order zero. Let $p=\sigma^{m}(P)$ and $p^{\prime}=\sigma^{m}\left(P^{\prime}\right)$ be the principal symbol of $P$ and $P^{\prime}$ respectively.

(i) Since $H_{p}+\gamma_{P}^{m-1} R=0$, and by assumption we know the principal symbols are same near the radial points by conjugating a zeroth order elliptic micro-differential operator $J$, i.e.,

$$
\begin{aligned}
\sigma^{m}\left(P^{\prime}\right)\left(\nu_{0}^{\prime}\right) & =\sigma^{m}\left(J^{-1} P J\right)\left(\nu_{0}\right) \\
& =\sigma^{0}\left(J^{-1}\right)\left(\nu_{0}\right) \sigma^{m}(P)\left(\nu_{0}\right) \sigma^{0}(J)\left(\nu_{0}\right) \\
& =\sigma^{m}(P)\left(\nu_{0}\right),
\end{aligned}
$$

then (i) is true.

(ii) If $A \in \mathcal{E}_{X}(n)$ is a $n$-th order micro-differential operator, notice that from Proposition 4.1 .2

$$
\begin{aligned}
& \sigma_{\text {sub }}^{m+n-1}(P A)=\sigma^{m}(P) \sigma_{\text {sub }}^{n-1}(A)+\sigma_{\text {sub }}^{m-1}(P) \sigma^{n}(A)+\frac{1}{2 i}\left\{\sigma^{m}(P), \sigma^{n}(A)\right\}, \\
& \sigma_{\text {sub }}^{m+n-1}(A P)=\sigma^{n}(A) \sigma_{\text {sub }}^{m-1}(P)+\sigma_{\text {sub }}^{n-1}(A) \sigma^{m}(P)+\frac{1}{2 i}\left\{\sigma^{n}(A), \sigma^{m}(P)\right\} .
\end{aligned}
$$

We know the principal symbol vanishes at radial point, then at the radial point we 
have

$$
\begin{aligned}
\sigma_{\mathrm{sub}}^{m+n-1}(P A) & =\sigma_{\mathrm{sub}}^{m-1}(P) \sigma^{n}(A) \\
& =\sigma^{n}(A) \sigma_{\text {sub }}^{m-1}(P) \\
& =\sigma_{\mathrm{sub}}^{m+n-1}(A P) .
\end{aligned}
$$

Suppose $A$ is elliptic, then at the radial point we have

$$
\begin{aligned}
\sigma_{\mathrm{sub}}^{m-1}\left(P^{\prime}\right)\left(\nu_{0}^{\prime}\right) & =\sigma_{\mathrm{sub}}^{m-1}\left(A^{-1} P A\right)\left(\nu_{0}\right) \\
& =\sigma_{\mathrm{sub}}^{m-1}\left(A A^{-1} P\right)\left(\nu_{0}\right) \\
& =\sigma_{\mathrm{sub}}^{m-1}(P)\left(\nu_{0}\right) .
\end{aligned}
$$

(iii) Assume we obtain two matrices $\frac{\gamma}{2}+A$ and $\frac{\gamma}{2}+A^{\prime}$ by linearizing the symplectic vector fields $H_{p}+\gamma R$ near $\nu_{0}$ and $H_{p^{\prime}}+\gamma R$ near $\nu_{0}^{\prime}$, respectively. Since the zeroth order microdifferential operator provided a morphism mapping $p$ to $p^{\prime}$, which induced a symplectic transformation maps $H_{p}$ to $H_{p^{\prime}}$. Then the associated contact transformation maps $V_{p}$ to $V_{p^{\prime}}$. Moreover, we have two contact transformations linearize $V_{p}$ and $V_{p^{\prime}}$ respectively. Hence there exist a linear symplectic map $V$ such that $A^{\prime}=V A V^{-1}$.

The rest of the proof is similar to the proof of the following corollary.

Corollary 4.3.2. Let $P \in \mathcal{E}(2)$ be a second order micro-differential operator on a real analytic manifold $M$. Let $\mathscr{M}$ be the $\mathcal{E}$-module associated with $P$, i.e., $\mathscr{M} \simeq \frac{\mathcal{E}}{\mathcal{E} P}$. Furthermore if $\nu_{0}=\left(x_{0}, \xi_{0}\right)$ is a generic radial point of $P$, then near $\left(x_{0}, \xi_{0}\right)$, via quantized contact transformation, one can transform $\mathscr{M}$ into the system

$$
\mathscr{N} \simeq \frac{\mathcal{E}}{\mathcal{E} P_{N}}
$$

and $P_{N}$ is the normal form of $P$ of form

$$
P_{N}=\left\langle A D_{x^{\prime}}, D_{x^{\prime}}\right\rangle+\left\langle B x^{\prime}, D_{x^{\prime}}\right\rangle D_{x_{n}}+\left\langle C x^{\prime}, x^{\prime}\right\rangle D_{x_{n}}^{2}+\gamma x_{n} D_{x_{n}}^{2}+\theta D_{x_{n}} .
$$

Here $A, B, C$ are constant $(n-1) \times(n-1)$ matrices, $A$ and $C$ are symmetric, $D_{x_{i}}=$ $\frac{1}{\sqrt{-1}} \frac{\partial}{\partial x_{i}}, x=\left(x^{\prime}, x_{n}\right), \gamma \in \mathbb{R}$ and $\theta \in \mathbb{C}$.

Proof. With slight modification of Guillemin and Schaeffer's proof, the essential idea works equally well here. Roughly, the steps are:

STEP I) One can conjugate $P$ into a micro-differential operator having the same principal symbol of the normal form $P_{N}$.

By direct computation, the normal form $P_{N}$ has a radial point at $\nu_{0}^{\prime}=\left(x_{0}^{\prime}, \xi_{0}^{\prime}\right)$ where $x_{0}^{\prime}=0, \xi_{0}^{\prime}=(0, \cdots, 0,1)$.

For technical reasons it is much easier to deal with first order operators, we consider a micro-differential operator $P \in \mathcal{E}_{X}(1)$, the normal form $P_{N}$ can be looked as a first order operator $P^{\prime}$ by the action of a micro-differential operator $\left(D_{x_{n}}\right)^{-1} \in \mathcal{E}_{\mathbb{R}^{n}}(-1)$, it 
is elliptic near the radial point $\nu_{0}^{\prime}$, then

$$
P^{\prime}=\left(D_{x_{n}}\right)^{-1}\left(P_{N}\right) \in \mathcal{E}_{\mathbb{R}^{n}}(1) .
$$

It is easy to see $\nu_{0}^{\prime}$ is also the radial point of $P^{\prime}$.

Let $p$ and $p^{\prime}$ be the principal symbol of $P$ and $P^{\prime}$, which by assumption are both homogeneous of degree one in $\xi$. Then $H_{p}$ and $H_{p^{\prime}}$ are homogeneous of degree zero on $T^{*} X$ and $T^{*} \mathbb{R}^{n}$, respectively. We can assign the Hamiltonian vector fields the related contact vector fields $V_{p}$ and $V_{p^{\prime}}$ on on $S^{*} X$ and $S^{*} \mathbb{R}^{n}$, respectively.

\begin{tabular}{l|cc|r}
\hline radial point & $\nu_{0}$ & & $\nu_{0}^{\prime}$ \\
\hline operator & $P$ & & $P^{\prime}$ \\
& $\uparrow$ & (a) & $\uparrow$ \\
principal symbol & $p$ & & $p^{\prime}$ \\
& $\uparrow$ & (b) & $\uparrow$ \\
Hamiltonian vector field & $H_{p}$ & & $H_{p^{\prime}}$ \\
& $\uparrow$ & (c) & $\uparrow$ \\
Contact vector field & $V_{p}$ & & $V_{p^{\prime}}$ \\
& $\uparrow$ & (d) & $\uparrow$ \\
Linear part & $V_{p}^{0}$ & & $V_{p^{\prime}}^{0}$ \\
\hline
\end{tabular}

In the above table, (a) and (b) are self-evident, (c) is canonical projection and (d) is the linearization of contact vector fields that we studied in chapter 3.

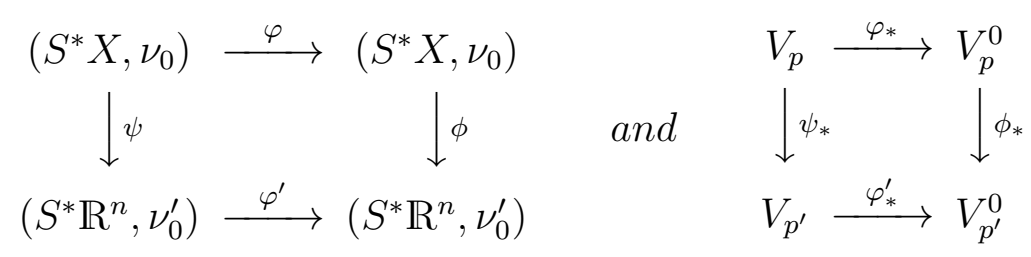

Choose suitable coefficients of $P_{N}$ such that there is a contact transformation $\phi$ such that $\phi_{*}$ can conjugate $V_{p}^{0}$ to $V_{p^{\prime}}^{0}$, and one knows that the maps $\varphi$ and $\varphi^{\prime}$, related to the linearization $\varphi_{*}$ and $\varphi_{*}^{\prime}$ of the contact vector fields $V_{p}$ and $V_{p^{\prime}}$, respectively, are also contact transformations. A combination of mappings $\psi \triangleq \varphi \circ \phi \circ \varphi^{\prime-1}$ shows us, near generical radial points, there is a contact transformations $\psi$ from $S^{*} X$ to $S^{*} \mathbb{R}^{n}$. The related symplectic transformation $\tilde{\psi}$ which maps $T^{*} X \backslash 0$ to $T^{*} \mathbb{R}^{n} \backslash 0$, i.e.,

$$
\psi(x, \xi)=(y, \eta) \leftrightarrow \tilde{\psi}(x, t \xi)=(y, t \eta), \forall t \in \in \mathbb{R} \backslash 0 .
$$

With the introduction of contact transformation and quantized contact transformation in A.3, one knows the contact transformation $\psi: S^{*} X \rightarrow S^{*} \mathbb{R}^{n}$ can be expressed by composition of two contact transformations with generating functions. To coincide with former introduction, we still use $\psi$ to denote the associated contact transformation from $P^{*} X$ to $P^{*} \mathbb{R}^{n}$.

Decompose $\psi=\pi_{2} \circ \pi_{1}^{-1}$ with a generating functions $\Gamma(x, r)$. Here we only need the existence of $\Gamma$ and we do not care what $\Gamma$ is. Let $\Lambda \subset X \times \mathbb{R}^{n}$ be non-singular 
hypersurface defined by $\Gamma(x, r)=0$, that is,

$$
\nabla_{(x, r)} \Gamma(x, r) \neq 0
$$

holds on $\Lambda$. Moreover, on $\Lambda$, one has

$$
\operatorname{det}\left(\begin{array}{cc}
0 & d_{y} \Gamma \\
d_{x} \Gamma & d_{x} d_{y} \Gamma
\end{array}\right) \neq 0 .
$$

And then

$$
\pi_{1}: P_{\Lambda}^{*}\left(X \times \mathbb{R}^{n}\right) \rightarrow P^{*} X
$$

and

$$
\pi_{2}: P_{\Lambda}^{*}\left(X \times \mathbb{R}^{n}\right) \rightarrow P^{*} \mathbb{R}^{n}
$$

Then we can lifting the contact transformation $\psi: P^{*} X \rightarrow P^{*} \mathbb{R}^{n}$ to the sheaves of germs of microdifferential operators $\mathcal{E}_{X} \rightarrow \mathcal{E}_{\mathbb{R}^{n}}$, via quantized contact transformation.

From theorem A.3.7, one can find an isomorphism between the two sheaves of germs of micro-differential operators $\pi_{2} \circ \pi_{1}^{-1} \mathcal{E}_{X}$ and $\mathcal{E}_{\mathbb{R}^{n}}$, as well as $\pi_{2} \circ \pi_{1}^{-1} \mathcal{E}_{X}(m)$ and $\mathcal{E}_{\mathbb{R}^{n}}(m)$, for any integer $m$. Moreover the isomorphism is unique up to an inner automorphism by an invertible microdifferential operator of order zero.

At least we can claim that we can find an invertible microdifferential operator of order zero, denote by $F$, such that

$$
\left\{\begin{array}{l}
P^{\prime}=F^{-1} P F+W, \\
\operatorname{ord}(W) \leq 1,
\end{array} \sigma^{1}(W)=0 \quad \text { near } \quad \nu_{0} .\right.
$$

STEP II) Deal with the lower order term.

Redefine the operator $P$ by $F^{-1} P F$, so far we have

$$
\sigma^{1}(P)=p=p^{\prime}=\sigma^{1}\left(P^{\prime}\right)
$$

We want to find an invertible micro-differential operator $Q$ of order zero, such that

$$
Q^{-1} P^{\prime} Q \simeq P
$$

That is the operator $Q^{-1} P^{\prime} Q$ and $P$ are microlocally equivalent in the sense of

$$
Q^{-1} P^{\prime} Q-P \in \cap_{m \in \mathbb{Z}} \mathcal{E}_{X}(m) .
$$

Now we are going to figure out $Q$. Consider the principal symbol of the zero order operator $Q^{-1} P^{\prime} Q-P$ near $\nu_{0}$. We have

$$
\begin{aligned}
\sigma^{0}\left(Q^{-1} P^{\prime} Q-P\right) & =\sigma^{0}\left(Q^{-1}\left(\left[P^{\prime}, Q\right]+P^{\prime}-P\right)\right) \\
& =\sigma^{0}\left(Q^{-1}\right) \sigma^{0}\left(\left[P^{\prime}, Q\right]\right)+\sigma^{0}\left(P^{\prime}\right)-\sigma^{0}(P) \\
& =\frac{\left\{p, q_{0}\right\}}{q_{0}}+\theta-s .
\end{aligned}
$$


Here $s$ is the zeroth order component of the total symbol of $P$, and $q_{0}$ the principal symbol $Q, \theta$ is the arbitrary constant in the first order term of $P_{N}$.

Write $q_{0}(x, \xi)=\exp \left(a_{0}(x, \xi)\right)$, and both of $q_{0}(x, \xi)$ and $a_{0}(x, \xi)$ are homogeneous of order zero in $\xi$. Let the above equation be zero then

$$
H_{p} a_{0}=s-\theta \triangleq b_{0}
$$

Suppose (4.8) can be solved, then choose $Q_{0}$ to be any micro-differential operator of order zero with principal symbol $q_{0}, Q_{0}^{-1} P^{\prime} Q_{0}-P$ will be of order -1 in a neighborhood of $\nu_{0}$.

Now again set $P^{\prime}$ to be $Q_{0}^{-1} P^{\prime} Q_{0}$, then $P^{\prime}-P \in \mathcal{E}_{\mathbb{R}^{n}}(-1)$. Consider a zero order operator $Q_{1}=I+A_{1}$ with $A_{1} \in \mathcal{E}_{X}(-1)$, then the principal symbol of the operator $Q_{1}^{-1} P^{\prime} Q_{1}-P$ is

$$
\begin{aligned}
\sigma^{-1}\left(Q_{1}^{-1} P^{\prime} Q_{1}-P\right) & =\sigma^{-1}\left(\left(\sum_{k \in \mathbb{N}}\left(-A_{1}\right)^{k}\right) P^{\prime}\left(I+A_{1}\right)-P\right) \\
& =\sigma^{-1}\left(\left[P^{\prime}, A_{1}\right]\right)+\sigma^{-1}\left(P^{\prime}-P\right) \\
& =\left\{p, a_{1}\right\}-b_{1},
\end{aligned}
$$

where $a_{1}$ is the principal symbol of $A_{1}$ and $b_{1}$ is the principal symbol of $P-P^{\prime}$. Suppose $\left\{p, a_{1}\right\}-b_{1}$ vanishes, then $a_{1}$ satisfies the equation

$$
H_{p} a_{1}=b_{1},
$$

repeat the above steps, after conjugating by a finite sequence of $Q_{i}^{\prime} s$, the principal symbol of $Q_{k}^{-1} P^{\prime} Q_{k}-P$ is

$$
\left\{p, a_{k}\right\}-b_{k}
$$

where $a_{k}$ is the principal symbol of $A_{k}=Q_{k}-I \in \mathcal{E}_{X}(-k)$ and $b_{k}$ is the principal symbol of $P^{\prime}-P$ near $\nu_{0}$.

If the above equation vanishes, then

$$
H_{p} a_{k}=b_{k}
$$

If it is satisfied, then we can construct $Q$ by combining all $Q_{i}$ 's, $i=0,1, \cdots$.

Consider the above all those unsolved equations

$$
H_{p} a_{k}=b_{k}, k=0,1, \cdots .
$$

If those above equations are solvable, then we can finish the proof.

Now we try to solve

$$
\left\{\begin{array}{l}
H_{p} a_{0}=s-\theta \\
H_{p} a_{k}=b_{k}
\end{array}\right.
$$


Since we have a diffeomorphism

$$
S^{*} X \times \mathbb{R}_{+} \cong T^{*} X \backslash 0
$$

we can treat a contact vector field $V_{p}$ as a vector field on $T^{*} X \backslash 0$,

$$
V_{p}=H_{p}+f R
$$

where $f$ is a homogeneous function of order zero on $\dot{T}^{*} X$, and $R$ is the radial vector field. At radial point, $f(0)=\lambda$. Let $c_{k}$ be the pull-back of $a_{k}$ via

$$
S^{*} X \rightarrow T^{*} X \backslash 0
$$

Since $a_{k}$ is homogenous of degree $-k, R a_{k}=-k a_{k}$, then it is equivalent to solve the following equations.

$$
\begin{cases}V_{p} c_{0} & =b_{0} \\ V_{p} c_{k}+k f c_{k} & =b_{k}\end{cases}
$$

where $f(0)=-\gamma$.

Replaying $S^{*} X$ by $\mathbb{R}^{2 n-1}$ with the contact structure

$$
d z+\frac{1}{2} \sum_{i=1}^{n-1}\left(y_{i} d x_{i}-x_{i} d y_{i}\right),
$$

by making a contact transformation and replay $V_{p}$ by some linear contract vector field $V_{P}^{0}$ on $\mathbb{R}^{2 n-1}$ of the form in section 3.3 . Due to Guillemin and Schaeffer's discussion in [22], we can solve it formally.

Roughly, let $S^{i}$ be the set of all polynomials in $x, y$ and $z$ which are linear combinations of polynomials of the form $z^{l} h(x, y)$, where $h$ is homogeneous in $x, y$ of order $m$ and $2 n+m=i$. So the vector field $V$ induces a linear map $V^{i}: S^{i} \rightarrow S^{i}$.

Lemma 4.3.3. To be able to solve the above equations formally (in power series about the origin), it is necessary and sufficient that $-k u$ not be an eigenvalue of $V^{i}$ for $i>0$ and for $k>0$.

The lemma shows that (4.11) can be solved up to error terms which vanish to order infinite ${ }^{2}$ at the origin of $\mathbb{R}^{n}$.

However, we do not need the argument as the smooth case, which need to discuss the error terms vanishing to infinite order at the origin. In fact the real analytic setting ensure that once we can solve the equations (4.11) formally, the problems have been solved.

We could have simpler normal form with additional conditions.

\footnotetext{
${ }^{2}$ A function $u(x)$ vanishes to infinite order at $x_{0}$ if for every positive integer $N, \lim _{x \rightarrow x_{0}} \frac{u(x)}{\left|x-x_{0}\right|^{N}}=0$ is true in a neighborhood of $x_{0}$.
} 
Corollary 4.3.4. Let $X$ be a n-dimensional real analytical manifold and $\mathscr{M}$ be a $\mathcal{E}_{X}$-module associated with a first order micro-differential operator $P \in \mathcal{E}_{X}(1)$. Let $\nu_{0}=\left(x_{0}, \xi_{0}\right)$ be a generical radial point. If $\nu_{0}$ is hyperbolic, then near $\nu_{0}$

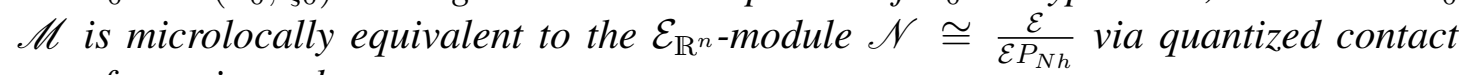
transformation, where

$$
P_{N h}=\left\langle\tilde{B} x^{\prime}, D_{x^{\prime}}\right\rangle+\gamma x_{n} D_{x_{n}}+\theta
$$

Here $\tilde{B}$ is a constant $(n-1) \times(n-1)$ matrix.

Corollary 4.3.5. Let $X$ be a n-dimensional real analytical manifold and $\mathscr{M}$ be $a$ $\mathcal{E}_{X}$-module associated with a second order micro-differential operator $P \in \mathcal{E}_{X}(2)$. Let $\nu_{0}=\left(x_{0}, \xi_{0}\right)$ be a generical radial point. If $\nu_{0}$ is elliptic, then near $\nu_{0} \mathscr{M}$ is microlocally

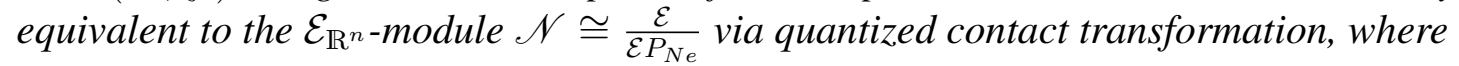

$$
P_{N e}=\left\langle\tilde{A} D_{x^{\prime}}, D_{x^{\prime}}\right\rangle+\left\langle\tilde{C} x^{\prime}, x^{\prime}\right\rangle D_{x_{n}}^{2}+\gamma x_{n} D_{x_{n}}^{2}+\theta D_{x_{n}}
$$

Here $\tilde{A}$ is a $(n-1) \times(n-1)$ identity matrix, $\tilde{C}=\left(\tilde{c}_{i}\right)$ is a $(n-1) \times(n-1)$ diagonal matrix with all non-vanishing entries larger than $\gamma^{2} / 16$.

Now we are going to prove the above corollaries. First we have:

Lemma 4.3.6. Let $P_{N}$ be the normal form of the operator $P$ with radial point $\nu_{0}=$ $\left(0 ; 0^{\prime}, 1\right)$, and $p$ is the principal symbol of $P_{N}$. Then the linear part $V_{p}^{0}$ of related symplectic vector field at the point $\nu_{0}$ is $\frac{\gamma}{2} I+A$, where

$$
A=\left(\begin{array}{c|c}
-b_{i j}+\frac{\gamma}{2} I & -2 c_{i j} \\
\hline 2 a_{i j} & b_{i j}+\frac{\gamma}{2} I
\end{array}\right)
$$

with respect to the symplectic basis

$$
\frac{\partial}{\partial \xi_{1}}, \cdots, \frac{\partial}{\partial \xi_{n-1}}, \frac{\partial}{\partial x_{1}}, \cdots, \frac{\partial}{\partial x_{n-1}} .
$$

Proof. At the radial point $\left(0 ; 0^{\prime}, 1\right)$, one has $d p \| \xi d x$ and

$$
H_{p}+\gamma \frac{\partial}{\partial \xi_{n}}=0
$$

From the discussion in section 4.2 , the space $d p=0$ divided by the space spanned by $H_{p}$ (i.e. $\widehat{E}_{\hat{\nu}_{0}}$ ) can be identified with the space spanned by the $\frac{\partial}{\partial \xi_{i}} s$ and $\frac{\partial}{\partial x_{i}} s, i, j<n$. Then

$$
p=\sum_{i, j<n}\left(a_{i j} \xi_{i} \xi_{j}+b_{i j} x_{i} \xi_{j} \xi_{n}+c_{i j} x_{i} x_{j} \xi_{n}^{2}\right)+\gamma x_{n} \xi_{n}^{2}
$$

and we have

$$
H_{p}=\sum_{i}\left(\frac{\partial p}{\partial \xi_{i}} \frac{\partial}{\partial x_{i}}-\frac{\partial p}{\partial x_{i}} \frac{\partial}{\partial \xi_{i}}\right) .
$$


Hence we obtain a homogeneous symplectic vector field $H_{p}+\gamma R$. Denote by $V_{p}$ the associated contact vector field on $\left(x_{1}, \cdots, x_{n}, \xi_{1}, \cdots, \xi_{n-1}\right)$ :

$$
V_{p}=\gamma \sum_{j} \xi_{j} \frac{\partial}{\partial \xi_{j}}+\sum_{i, j<n}\left(2 a_{i j} \xi_{i} \frac{\partial}{\partial x_{j}}+b_{i j} x_{i} \frac{\partial}{\partial x_{j}}-b_{i j} \xi_{i} \frac{\partial}{\partial \xi_{j}}-2 c_{i j} x_{i} \frac{\partial}{\partial \xi_{j}}\right)+\cdots
$$

where the dots are quadratic terms in $x_{1}, \cdots, x_{n-1}, \xi_{1}, \cdots, \xi_{n-1}$ and terms involved with $\partial_{x_{n}}$. Therefore the linear part $V_{p}^{0}$ of $V_{p}$ related to $\left(x^{\prime}, \xi^{\prime}\right)$ is give by

$$
\left(\xi^{\prime}, x^{\prime}\right)\left(\frac{\gamma}{2} I+A\right)\left(\partial_{\xi^{\prime}}, \partial_{x^{\prime}}\right)^{T}
$$

which can be seen from the above formula. Here $x^{\prime}=\left(x_{1}, \cdots, x_{n-1}\right)$, and $\xi^{\prime}=$ $\left(\xi_{1}, \cdots, \xi_{n-1}\right)$.

To prove the Corollary 4.3.5, if $P$ has the normal form $P_{N e}$, according to Lemma 4.3.6, the matrix $A$ is given by

$$
\left(\begin{array}{cc}
\frac{\gamma}{2} & -2 \tilde{c}_{i} \\
2 & -\frac{\gamma}{2}
\end{array}\right) \text { or }\left(\begin{array}{cc}
\frac{\gamma}{2} & 2 \tilde{c}_{i} \\
-2 & -\frac{\gamma}{2}
\end{array}\right)
$$

The eigenvalues of $A$ is purely imaginary if and only if the determinant of the matrix

$$
-\frac{\gamma^{2}}{4}+4 \tilde{c}_{i}
$$

is greater than zero, or $\tilde{c}_{i}>\frac{\gamma^{2}}{16}$. Conversely, every elliptic case can be obtained by this way.

To prove the Corollary 4.3.4 if $P$ is microlocally equivalent to the normal form

$$
P_{N h}=\sum_{i, j<n} b_{i j} x_{i} \frac{\partial}{\partial x_{j}} \frac{\partial}{\partial x_{n}}+\gamma x_{n} \frac{\partial^{2}}{\partial x_{n}^{2}}+\theta \frac{\partial}{\partial x_{n}}
$$

near the radial point. According to Lemma 4.3.6, the matrix $A$ is given by

$$
\left(\begin{array}{c|c}
-b_{i j}+\frac{\gamma}{2} & 0 \\
\hline 0 & b_{i j}-\frac{\gamma}{2}
\end{array}\right)
$$

Conversely, every hyperbolic case can be obtained by this way.

Go back to our normal form $P_{N}$ in Corollary 4.3.2.

Remark 4.3.7. Notice that $P_{N}$ is invariant invariant up to some constant $\kappa$ under the coordinates transformation:

$$
\left(x^{\prime}, x_{n}\right) \mapsto\left(\kappa x^{\prime}, \kappa^{2} x_{n}\right),
$$

which will help to construct special form of solutions. 
Remark 4.3.8. The subprincipal symbol of $P_{N}$ is

$$
\sigma_{\text {sub }}^{1}\left(\nu_{0}\right)=\theta+\frac{\sqrt{-1}}{2}(\operatorname{tr}(B)+2 \gamma)
$$

\subsection{Projected null bicharacteristics}

First we recall the definition of a bicharacteristic strip in the classical theory of partial differential equations.

Definition 4.4.1. Let $p_{m}(x, \xi)$ be the principal symbol of a linear differential operator $P\left(x, D_{x}\right)$. An integral curve $(x(t), \xi(t))$ of

$$
\frac{d x_{1}}{\frac{\partial p_{m}}{\partial \xi_{1}}}=\cdots=\frac{d x_{n}}{\frac{\partial p_{m}}{\partial \xi_{n}}}=\frac{d \xi_{1}}{-\frac{\partial p_{m}}{\partial x_{1}}}=\cdots=\frac{d \xi_{n}}{\frac{\partial p_{m}}{\partial x_{n}}}
$$

with the property $p_{m}(x(t), \xi(t))=0$ is said to be a bicharacteristic strip of the equation $P u=0$. The image $\{x(t)\}$ of the projection of a bicharacteristic strip onto the base space is call the bicharacteristic curve.

Let $P$ be the microdifferential operator in our problem, $p$ is the principal symbol of $P$, and

$$
H_{p}=\sum_{i=1}^{n}\left(\frac{\partial p}{\partial \xi_{i}} \frac{\partial}{\partial x_{i}}-\frac{\partial p}{\partial x_{i}} \frac{\partial}{\partial \xi_{i}}\right)
$$

is the Hamiltonian vector field. Consider the integral curves $(x(s), \xi(s))$ of the Hamiltonian flow:

$$
\begin{cases}\frac{d x_{i}}{d s}=\frac{\partial p}{\partial \xi_{i}}, & 1 \leq i \leq n \\ \frac{d \xi_{i}}{d s}=-\frac{\partial p}{\partial x_{i}}, & 1 \leq i \leq n\end{cases}
$$

passing through $\left(x^{0}, \xi^{0}\right)$, such that

$$
p\left(x^{0}, \xi^{0}\right)=0, \quad \xi^{0} \neq 0,
$$

that is, $\left(x^{0}, \xi^{0}\right) \in \operatorname{Char}(P)$.

By calculation on the normal form we obtained in the section 4.3 , we can set the radial point to be $\left(0 ; 0^{\prime}, 1\right){ }^{3}$. We project these bicharacteristics into the plane $\left\{\xi_{n}=1\right\}$ by

$$
\begin{aligned}
T^{*} \mathbb{R}^{n} \backslash 0 & \rightarrow S^{*} \mathbb{R}^{n} \\
\left(x_{1}, \cdots, x_{n} ; \xi_{1}, \cdots, \xi_{n-1}, \xi_{n}\right) & \mapsto\left(x_{1}, \cdots, x_{n} ; \frac{\xi_{1}}{\xi_{n}}, \cdots, \frac{\xi_{n-1}}{\xi_{n}}, 1\right) .
\end{aligned}
$$

\footnotetext{
${ }^{3}$ For a point $(x ; \xi) \in S^{*} \mathbb{R}^{n}$, we have $(x ; \xi)=\left(x_{1}, \cdots, x_{n} ; \xi_{1}, \cdots, \xi_{n}\right)$, and $x^{\prime}=\left(x_{1}, \cdots, x_{n-1}\right)$ and $\xi^{\prime}=\left(\xi_{1}, \cdots, \xi_{n-1}\right)$.
} 
Then the flow defined above satisfies

$$
\left\{\begin{aligned}
\frac{d x_{i}}{d s} & =\frac{\partial p}{\partial \xi_{i}}, \quad 1 \leq i<n \\
\frac{d\left(\frac{\xi_{n-1}}{\xi_{n}}\right)}{d s} & =\frac{-\frac{\partial p}{\partial x_{i}} \xi_{n}+\xi_{i} \frac{\partial p}{\partial x_{n}}}{\xi_{n}^{2}}, \quad 1 \leq i \leq n-1
\end{aligned}\right.
$$

passing through $\left(x^{0}, \xi^{0}\right) \neq\left(0 ; 0^{\prime}, 1\right)$, with $p\left(x^{0}, \xi^{0}\right)=0$.

Since

$$
\left(\frac{\partial p}{\partial \xi_{n}}\right)\left(0 ; 0^{\prime}, 1\right)=-\frac{d \xi_{n}}{d s}\left(0 ; 0^{\prime}, 1\right) \neq 0
$$

by assumption that at $\left(0 ; 0^{\prime}, 1\right)$ the Hamiltonian vector field is radial. A point in the Characteristics should satisfies the equation

$$
p(x, \xi)=0
$$

then near the radial point we can determine the value of $x_{n}$ in terms of $x_{i}^{\prime} s$ and $\xi_{i}^{\prime} s$ for $1 \leq i \leq n-1$. In sum, the projected bicharacteristic flow has an isolated zero at $\left\{\left(0 ; 0^{\prime}, 1\right)\right\} \in S^{*} \mathbb{R}^{n}$.

From section 3.3, we assume the symplectic matrix $A$ is semi-simple and for any eigenvalue $\lambda$ of $A$, from (3.3) we have

$$
\operatorname{Re} \lambda \neq \frac{1}{2}
$$

Moreover, we have generical assumption (non-resonant condition) for radial points in Definition 4.2.4.

The projected null bicharacteristics on $\left\{\xi_{n}=1\right\}$ play a significant role in analyzing the propagation of singularities. In this section we give an explicit way to computer the systems that determine the projected bicharacteristic manifold.

In two dimensional case, we have the normal form

$$
P_{N}=a D_{x}^{2}+b x D_{x} D_{y}+c x^{2} D_{y}^{2}+\gamma y D_{y}^{2}+\theta D_{y} .
$$

The principal symbol is

$$
\sigma(P)=a \xi^{2}+b x \xi \eta+c x^{2} \eta^{2}+\gamma y \eta^{2} .
$$

By the radial map $(x, y, \xi, \eta) \rightarrow(x, y, \tilde{\xi}, 1)$, where $\tilde{\xi}=\frac{\xi}{\eta}$, we project the bicharacteristics into the plane $\eta=1$. Along the projected null bicharacteristics we have

$$
y=-\left(a \xi^{2}+b x \xi+c x^{2}\right)
$$

hence the variable $y$ can be determined from a knowledge of $x$ and $\xi$ and we ignore this variable. A simple computation shows the following systems governs the projected null 
bicharacteristics:

$$
\left(\begin{array}{c}
\dot{x} \\
\dot{\tilde{\xi}}
\end{array}\right)=\left(\begin{array}{cc}
b & 2 a \\
-2 c & \gamma-b
\end{array}\right)\left(\begin{array}{c}
x \\
\tilde{\xi}
\end{array}\right)
$$

Let $\gamma=1$, we have two eigenvalues of the above system

$$
\lambda_{ \pm}=\frac{1 \pm \sqrt{1+4\left(b^{2}-b-4 a c\right)}}{2}=\frac{1 \pm \sqrt{\left.(2 b-1)^{2}-16 a c\right)}}{2} .
$$

Here $b \neq \frac{1}{2}$ to make the matrix is hyperbolic (linearization). The behavior of the projected null bicharacteristics change while the parameters $a, b$ and $c$ change,

(a) $\frac{(2 b-1)^{2}-1}{16}<a c<\frac{(2 b-1)^{2}}{16}$, two positive eigenvalues.

(b) $a c>\frac{(2 b-1)^{2}}{16}$, two conjugate complex with positive real part.

(c) $a c<\frac{(2 b-1)^{2}-1}{16}$, one positive eigenvalue and one negative.

The flows of projected null bicharacteristics are of three types: the bifurcation phenomenon is going to happen when the parameters are changing.

One can discuss the normal form more specifically, for the two dimensional elliptic case, the normal form is

$$
P=D_{x}^{2}+\left(y+c x^{2}\right) D_{y}^{2}+\theta D_{y}, \quad c>\frac{1}{16} .
$$

and we have the following system

$$
\left(\begin{array}{c}
\dot{x} \\
\dot{\tilde{\xi}}
\end{array}\right)=\left(\begin{array}{cc}
0 & -2 \\
2 c & -1
\end{array}\right)\left(\begin{array}{l}
x \\
\tilde{\xi}
\end{array}\right)
$$

will govern the projected bicharacteristic manifolds. The eigenvalues of the matrix is $\frac{1 \pm \sqrt{1-16 c}}{2}$.

And for the hyperbolic saddle case, the operator is of normal form:

$$
P=b x D_{x}+\gamma y D_{y}+\theta, \quad \text { where } \quad b<0 \quad \text { and } \quad b>1 .
$$

set $\gamma=1$, then we have the following system

$$
\left(\begin{array}{c}
\dot{x} \\
\dot{\tilde{\xi}}
\end{array}\right)=\left(\begin{array}{cc}
b & 0 \\
0 & 1-b
\end{array}\right)\left(\begin{array}{c}
x \\
\tilde{\xi}
\end{array}\right)
$$

that will govern the projected bicharacteristic manifolds. We see from the matrix that the case $b>1$ and $b<0$ are equivalent, then we can consider the operator as

$$
P=x D_{x}-\lambda y D_{y}+\alpha, \text { where } \lambda>0 .
$$




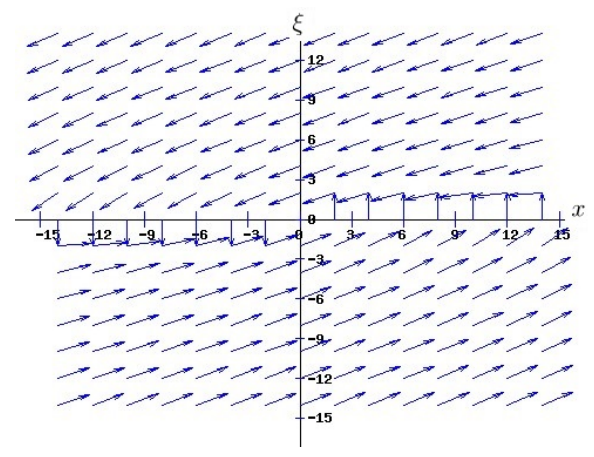

(a) Hyperbolic repelling

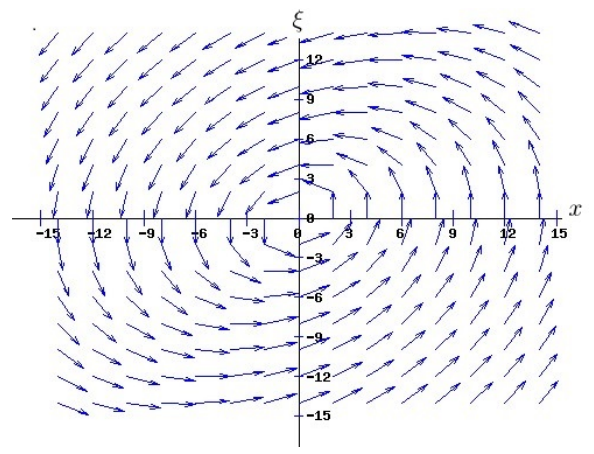

(b) Elliptic

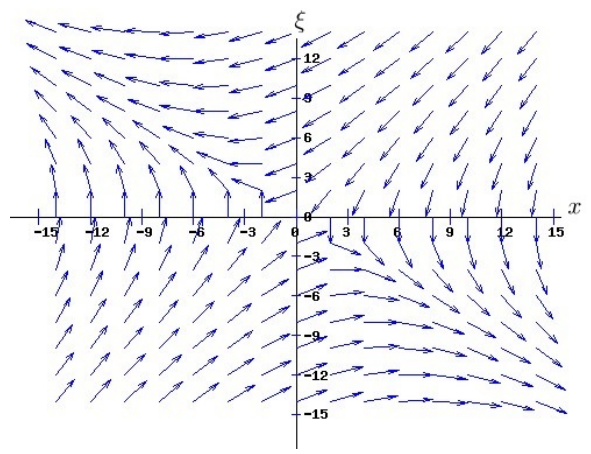

(c) Hyperbolic saddle

Figure 4-1: Flows of projected null bicharacteristics in 2D

One has $x(t)=C e^{t}$ and $\tilde{\xi}(t)=C e^{-(1+\lambda) t}$ from above. Then one have four projected null bicharacteristics that go asymptotically to $(0,0,0,1)$.

$$
\begin{gathered}
x= \pm e^{t}, y=\xi=0, \eta=1, \quad \text { as } \quad t \rightarrow-\infty, \\
x=y=0, \xi= \pm e^{-(1+\lambda) t}, \eta=1, \quad \text { as } \quad t \rightarrow+\infty .
\end{gathered}
$$

We will discuss the propagation of singularities in section 5.4 .

Moreover, for three dimensional case, there are eight cases to be discussed (see Chapter 6), the normal form near a hyperbolic radial point can be written as

$$
P=b_{11} x D_{x}+b_{12} x D_{y}+b_{21} y D_{x}+b_{22} y D_{y}+\gamma z D_{z}+\theta,
$$


By choose suitable local coordinates, we have even simple one

$$
P=b_{1} x D_{x}+b_{2} y D_{y}+\gamma z D_{z}+\theta
$$

Here $b_{1}$ and $b_{2}$ are eigenvalues of the matrix

$$
\left(\begin{array}{ll}
b_{11} & b_{12} \\
b_{21} & b_{22}
\end{array}\right) \text {. }
$$

The projected null bicharacteristic manifold is governed by the following system

$$
\left(\begin{array}{c}
\dot{x} \\
\dot{y} \\
\dot{\xi} \\
\dot{\eta}
\end{array}\right)=\left(\begin{array}{cccc}
b_{1} & 0 & 0 & 0 \\
0 & b_{2} & 0 & 0 \\
0 & 0 & \gamma-b_{1} & 0 \\
0 & 0 & 0 & \gamma-b_{2}
\end{array}\right)\left(\begin{array}{l}
x \\
y \\
\xi \\
\eta
\end{array}\right)
$$

One has $x(t)=C e^{b_{1} t}, y(t)=C e^{b_{2} t}, \xi(t)=C e^{\left(\gamma-b_{1}\right) t}$ and $\eta(t)=C e^{\left(\gamma-b_{2}\right) t}$ from above. When $b_{1}$ and $b_{2}$ take different values, one has the following 24 possible projected null bicharacteristics that tend asymptotically to $(0,0,0,0,0,1)$. Type (i) and (ii) involve in the case $\lambda_{1}$ does not satisfy the the attracting/repelling condition (6.2), type (iii) and (iv) involve in the case $\lambda_{2}$ does not satisfy the condition (6.2), and the other four types involve in the case both $\lambda_{1}$ and $\lambda_{2}$ do not satisfy condition 6.2).

\begin{tabular}{l|c|c|c|c|c|c}
\hline type & $\mathrm{x}$ & $\mathrm{y}$ & $\mathrm{z}$ & $\xi$ & $\eta$ & $\zeta$ \\
\hline $\mathrm{i}$ & $\pm e^{b_{1} t}$ & 0 & 0 & 0 & 0 & 1 \\
\hline ii & 0 & 0 & 0 & $\pm e^{\left(\gamma-b_{1}\right) t}$ & 0 & 1 \\
\hline iii & 0 & 0 & 0 & 0 & $\pm e^{\left(\gamma-b_{2}\right) t}$ & 1 \\
\hline iv & 0 & $\pm e^{b_{2} t}$ & 0 & 0 & 0 & 1 \\
\hline $\mathrm{v}$ & $\pm e^{b_{1} t}$ & $\pm e^{b_{2} t}$ & 0 & 0 & 0 & 1 \\
\hline vi & $\pm e^{b_{1} t}$ & 0 & 0 & 0 & $\pm e^{\left(\gamma-b_{2}\right) t}$ & 1 \\
\hline vii & 0 & $\pm e^{b_{2} t}$ & 0 & $\pm e^{\left(\gamma-b_{1}\right) t}$ & 0 & 1 \\
\hline viii & 0 & 0 & 0 & $\pm e^{\left(\gamma-b_{1}\right) t}$ & $\pm e^{\left(\gamma-b_{2}\right) t}$ & 1 \\
\hline
\end{tabular}

Table 4.1: List of projected null bicharacteristics

For three dimensional case of mixed type, we have normal form

$$
P= \pm\left(D_{x}^{2}+c x^{2} D_{z}^{2}\right)+b y D_{y} D_{z}+\gamma z D_{z}^{2}+\theta D_{z}
$$

where $c>\frac{\gamma^{2}}{16}$.

Without loss of generality, we consider

$$
P=D_{x}^{2}+c x^{2} D_{z}^{2}+b y D_{y} D_{z}+\gamma z D_{z}^{2}+\theta D_{z},
$$


We have the following systems that determine the projected characteristics:

$$
\left(\begin{array}{c}
\dot{x} \\
\dot{y} \\
\dot{\xi} \\
\dot{\eta}
\end{array}\right)=\left(\begin{array}{cccc}
0 & 0 & 2 & 0 \\
0 & b & 0 & 0 \\
-2 c & 0 & \gamma & 0 \\
0 & 0 & 0 & \gamma-b
\end{array}\right)\left(\begin{array}{l}
x \\
y \\
\xi \\
\eta
\end{array}\right)
$$

And the four eigenvalues are $b, \gamma-b, \frac{\gamma \pm \sqrt{\gamma^{2}-16 c}}{2}$.

The qualitative behavior of the solutions of the above system depends on the value of $b$ and $c$, set $\gamma=1$,

(a) for $0<b<1, c>\frac{1}{16}$. In this case, two of the four eigenvalues are positive, and two are conjugated complex number with positive real part.The projected bicharacteristics tending to the origin as $t \rightarrow-\infty$ and to infinity as $t \rightarrow \infty$. Notice that in $(x, \xi)$ variables they are spirals;

(b) for $b<0$ or $b>1$ (such two cases are equivalent, here we consider $b<0$ without loss of generality), $c>\frac{1}{16}$, two of the four eigenvalues are real and of opposite sign, and two are conjugated complex number with positive real part. The projected bicharacteristics have saddle point behavior in $(y, \eta)$ near the origin, and in $(x, \xi)$ variables they are spirals. Rewrite the system as:

$$
\left(\begin{array}{c}
\dot{x} \\
\dot{\xi} \\
\dot{y} \\
\dot{\eta}
\end{array}\right)=\left(\begin{array}{cc|cc}
0 & 2 & 0 & 0 \\
-2 c & \gamma & 0 & 0 \\
\hline 0 & 0 & b & 0 \\
0 & 0 & 0 & \gamma-b
\end{array}\right)\left(\begin{array}{c}
x \\
\xi \\
y \\
\eta
\end{array}\right)
$$

We can study the system separately via subspaces in coordinated $(x, \xi)$ and $(y, \eta)$.

Remark 4.4.2. The above discussion inspires us to study the behaviors of sub-systems to understand the original system. 


\section{Chapter 5}

\section{Analytic singularities in two dimensions}

In this chapter we completely studied the analytic singularities of solutions of our problem in two dimensional space. Near an elliptic radial point or a hyperbolic attracting (resp. repelling) radial point, there are solutions with minimal analytic singularity, i.e. the radial direction. Near a hyperbolic saddle radial point, if the radial direction is contained in the analytic wavefront set of a solution, then at least one of the projected null bicharacteristics running into the radial direction will be contained in the analytic wavefront set of the solution.

\subsection{Main results}

Consider our problem in two dimensional case. Let $\nu_{0}=\left(x_{1}, x_{2}, \xi_{1}, \xi_{2}\right)$ be an isolated generic radial point of $P$. In a conical neighborhood of the ray

$$
\Upsilon=\left\{\left(x_{1}, x_{2}, t \xi_{1}, t \xi_{2}\right): t>0\right\}
$$

the operator $P$ has the following normal form

$$
P_{N}=\partial_{x_{1}}^{2}+b x_{1} \partial_{x_{1}} \partial_{x_{2}}+\left(c x_{1}^{2}+\gamma x_{2}\right) \partial_{x_{2}}^{2}+\theta \partial_{x_{2}} .
$$

Here $\gamma \in \mathbb{R} \backslash 0$ and $\theta \in \mathbb{C}$. The radial points of $P_{N}$ are

$$
\{(0,0,0,1)\} \in S^{*} \mathbb{R}^{2} .
$$

We want to study the analytic singularities of the solutions of $P u=f$ near radial point $\nu_{0}$, where $f$ is a real analytic function. Equivalently, we will work on the normal form $P_{N}$ for simplicity.

Definition 5.1.1. ([GS] [22]) Let $V_{p}$ be the linear contact vector field associated with the operater $P$ and let $z_{0}$ be the zero of $V_{p}$ corresponding to the radial point $\nu_{0}$. Say $\nu_{0}$ is attracting (or repelling) if the flow of $V_{p}$ has source (or sink) at $z_{0}$. 
In our case, the flow of $V_{p}$ depends on the value of $\gamma$ and the eigenvalues of $A$.

We are going to study the analytic singularities in two dimensional case in this section. The eigenvalues of the symplectic matrix $\frac{\gamma}{2} I+A$ are of three types as in the Figure 5-1.

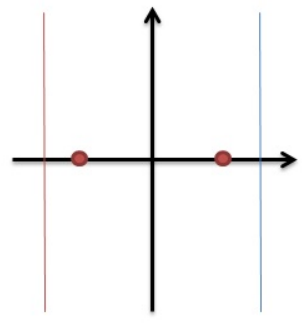

(a) Hyperbolic attracting/repelling

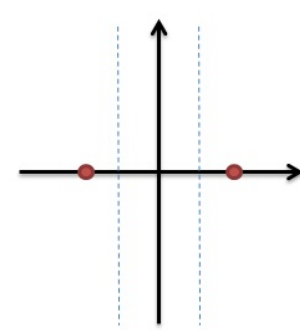

(b) Hyperbolic saddle

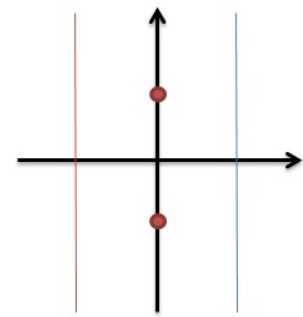

(c) Elliptic attracting/repelling

Figure 5-1: Classification of eigenvalues of $A$ for $2 D$

(a) two real numbers with same sign, the radial point is hyperbolic attracting (resp. repelling),

(b) two real numbers with different sign, the radial point is hyperbolic saddle,

(c) two pure imaginary numbers, the radial point is elliptic attracting (resp. repelling).

Remark 5.1.2. In Figure 5-1, “•" means the position of a eigenvalue of $A$, and the vertical lines mean the possible values of $\frac{\gamma}{2}$.

In the cases (a) and (c), the eigenvalues related to the linear part $\frac{\gamma}{2} I+A$ of the vector field are in common that, they convex hull of the four eigenvalues does not contain zero. That is, the eigenvalues are of Poincaré type ${ }^{1}$, while the eigenvalues of the case (b) are of Siegel type.

To continue our statement, without loss of generality, we set $\gamma=1$.

Lemma 5.1.3. Let $P$ be a second order microdifferential operator defined on a two dimensional real analytic manifold $M$, with real-analytic coefficients, real principal symbol $p$, and simple characteristics. Near a generic radial point $\nu_{0}=\left(x_{0}, \xi_{0}\right)$, we have

(i) if $\left(x_{0}, \xi_{0}\right)$ is an elliptic radial point, then near $\nu_{0}, P$ has the normal form

$$
P_{N e}=\partial_{x_{1}}^{2}+\left(x_{2}+c x_{1}^{2}\right) \partial_{x_{2}}^{2}+\theta \partial_{x_{2}}, \quad c>\frac{1}{16}, \theta \in \mathbb{C} .
$$

\footnotetext{
${ }^{1}$ See Definition A.4.5.
} 
(ii) If $\left(x_{0}, \xi_{0}\right)$ is a hyperbolic attracting (resp. repelling) radial point, then near $\nu_{0}$, $P$ has the normal form

$$
P_{N h}=b x_{1} \partial_{x_{1}}+x_{2} \partial_{x_{2}}+\theta, \quad 0<b<1, \theta \in \mathbb{C}
$$

(iii) If $\left(x_{0}, \xi_{0}\right)$ is a hyperbolic saddle radial point, then near $\nu_{0}, P$ has the normal form

$$
P_{N h}=b x_{1} \partial_{x_{1}}+x_{2} \partial_{x_{2}}+\theta, \quad b<0 \quad \text { or } \quad b>1, \theta \in \mathbb{C} .
$$

Remark 5.1.4. We use $\partial_{x}$ instead of $D_{x}=\frac{1}{\sqrt{-1}} \frac{\partial}{\partial x}$ for simplicity of the following discussion..

By direct computation of the matrix $A$, one can see the two cases of $b<0$ and $b>1$ in (iii) are equivalent. Instead of the normal form in (iii), we prefer to consider the normal form

$$
P_{N h}=x_{1} \partial_{x_{1}}-\lambda x_{2} \partial_{x_{2}}+\theta, \quad \text { for } \quad \lambda>0
$$

Moreover, the radial point of our normal form is $(0,0 ; 0,1) \in S^{*} \mathbb{R}^{2}$.

Theorem 5.1.5. Let $P$ be a microdifferential operator satisfies the conditions in Lemma 5.1 .3 and $P_{N}$ be its normal form. Consider the equation

$$
P_{N} u=f
$$

in space of hyperfunctions, where $f$ is a real analytic function. Suppose the dimension $n=2, \nu_{0}=(0,0 ; 0,1)$ and $\gamma=1$. Set $\mathbb{R}_{+} \nu_{0}=\left\{\left(0 ; 0^{\prime}, \eta\right) \mid \eta>0\right\}$. Then

(i) when the radial point $\nu_{0}$ is elliptic, there is a solution of the equation (5.3) with $f \in C^{\infty}(X)$ such that the minimal wave front set is $\mathrm{WF}_{a}(u)=\mathbb{R}_{+} \nu_{0}$.

(ii) when the radial point $\nu_{0}$ is hyperbolic attractor/repellor, there is a solution of the equation (5.3) with $f \in C^{\infty}(X)$ such that the minimal wave front set is $\mathrm{WF}_{a}(u)=\mathbb{R}_{+} \nu_{0}$.

(iii) when the radial point $\nu_{0}$ is hyperbolic saddle, and if $\nu_{0} \in \mathrm{WF}(u)$, then one of the four projected null bicharacteristic curves ${ }^{2}$ which go asymptotically to $\nu^{0}$ must intersect $\mathrm{WF}_{a}(u)$ :

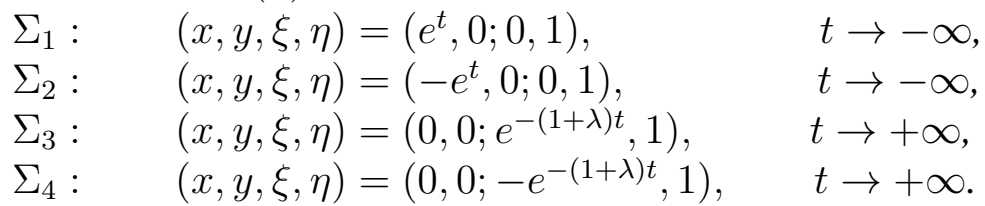

That is, there are 3 minimal wave front sets: $\Sigma_{1} \cup \mathbb{R}_{+} \nu_{0}, \Sigma_{2} \cup \mathbb{R}_{+} \nu_{0}$ and $\Sigma_{3} \cup$ $\Sigma_{4} \cup \mathbb{R}_{+} \nu_{0}$.

Let $P_{N}$ be the suitable normal form for $P$ near the radial point $\nu^{0}$, and let $F$ be a microdifferential operator such that the quantized contact transformation satisfies

$$
P F-F P_{N}=E,
$$

\footnotetext{
${ }^{2}$ See section 4.4 for detail.
} 
where $E$ has the property that $\nu_{0} \notin \mathrm{WF}_{a}(E u)$ for any $u \in \mathscr{B}(X)$. Suppose $F$ is the associated with the canonical contact transformation $\psi$. Choose two conical neighborhoods $N_{1}$ and $N_{2}$ of $(0,0,0,1)$ with $N_{1} \subset N_{2}$, and arrange that

$$
\begin{aligned}
& \mathrm{WF}_{a}(F u) \subset \psi\left(N_{2} \cap \mathrm{WF}_{a}(u)\right) \\
& \mathrm{WF}_{a}(E u) \subset \psi\left(\left(N_{2} \backslash N_{1}\right) \cap \mathrm{WF}_{a}(u)\right) .
\end{aligned}
$$

That is, for a inhomogeneous equation $P u=f$, we can consider the homogeneous equation $P_{N} u=0$. We will need it in next section.

\subsection{Elliptic case}

In this section, we are going show near an elliptic radial point, there is a hyperfunction solution $u$ of the equation (5.3) such that $u$ has the minimal analytic singularity, i.e., $\mathrm{WF}_{a}(u)=\{(0,0,0, \eta) \mid \eta>0\}$.

Theorem 5.2.1. Let $M$ be a two dimensional real analytic manifold and let $P(x, D)$ be a differential operator defined on $M$ and $p$ is the principal symbol of $P$, let $u$ and $f$ be hyperfunctions which satisfy

$$
P(x, D) u(x)=f(x) .
$$

Then the following inclusion holds:

$$
\mathrm{WF}_{a}(u) \subset\left\{(x, \xi) \in T^{*} M \backslash 0 \mid p(x, \xi)=0\right\} \cup \mathrm{WF}_{a}(f) .
$$

In particular, if $P$ is elliptic at a point $x_{0} \in M$, then $f$ is real analytic at $x_{0}$ implies $u$ is real analytic at $x_{0}$.

Before giving our theorem, first we have the following lemma:

Lemma 5.2.2. Suppose $\mu \in \mathbb{C}$ and let $u(x) \in \mathcal{A}\left(\mathbb{R}^{2} \backslash\{0\}\right)$ be homogeneous of degree $\mu$ under coordinates transformation $u\left(c x_{1}, c^{2} x_{2}\right)=c^{\mu} u\left(x_{1}, x_{2}\right)$ for all $c \neq 0$. Provided that $\mu \neq-3,-4,-5, \cdots$, there is a unique hyperfunction $\tilde{u}(x) \in \mathscr{B}\left(\mathbb{R}^{2}\right)$ with the homogeneity property such that $\tilde{u}(x)=u(x)$ on $\mathbb{R}^{2} \backslash\{0\}$.

Proof. In Guillemin-Schaeffer's paper [22], they already proved the existence and uniqueness of a distribution $\tilde{u}(x) \in \mathscr{D}^{\prime}\left(\mathbb{R}^{2}\right)$ with the homogeneity property such that $\tilde{u}(x)=u(x)$ on $\mathbb{R}^{2} \backslash\{0\}$.

For our case, we only need to notice that a hyperfunction whose support is the origin can be written as

$$
\mathscr{B}[\{0\}]=\left\{\sum_{m=0}^{\infty} c_{m} \delta^{(m)}(x) \mid c_{m} \in \mathbb{C}, \limsup _{m \rightarrow \infty} \sqrt[m]{m !\left|c_{m}\right|}=0\right\} .
$$

If the above sum is a finite sum, then such a hyperfunction is a distribution. Moreover, to 
satisfy the homogeneity condition, such hyperfunctions can not be written as an infinite sum.

Theorem 5.2.3. Let $P$ be a microdifferential operator satisfies conditions in Lemma 5.1.3 then near an elliptic radial point, there exist a hyperfunction solution $u$ of the equation $P_{N e} u=f$ such that $u$ has minimal analytic singularity in radial direction.

Proof. Notice that a slight modification of Guillemin and Schaeffer [22]'s proof works equally well for our case.

Let $L$ be a normal form of $P$ near radial point, first we consider the homogeneous equation

$$
L u(x)=\partial_{x_{1}}^{2} u(x)+\left(x_{2}+c x_{1}^{2}\right) \partial_{x_{2}}^{2} u(x)+\theta \partial_{x_{2}} u(x)=0, \quad c>\frac{1}{16}
$$

is invariant up to some constant $\kappa$ under the coordinates transformation

$$
\left(x_{1}, x_{2}\right) \rightarrow\left(\kappa x_{1}, \kappa^{2} x_{2}\right),
$$

that is, if $u\left(x_{1}, x_{2}\right)$ is a solution of 5.5$)$, then $u\left(\kappa x_{1}, \kappa^{2} x_{2}\right)$ is also a solution. Moreover, let $\kappa=-1$, then if $u\left(x_{1}, x_{2}\right)$ is a solution, so does $u\left(-x_{1}, x_{2}\right)$. Suppose $u\left(x_{1}, x_{2}\right)$ is homogeneous of degree $\mu$ under the transformation (5.6).

Denote $y=\frac{x_{2}}{x_{1}^{2}}$ and set $u(x)=x_{1}^{\mu} v(y)$, plug into 5.5$)$, one has

$$
x_{1}^{\mu-2}\left\{\left(4 y^{2}+y+c\right) v^{\prime \prime}(y)+y[(6-4 \mu) z+\theta] v^{\prime}(y)+\mu(\mu-1) v(y)\right\}=0 .
$$

Denote (5.7) by

$$
x_{1}^{\mu-2} Q v(y)=0,
$$

where $Q$ is a second order ordinary differential operator. If $v(y)$ is a solution of $Q v(y)=$ 0 , then $u(x)=x_{1}^{\mu} v\left(\frac{x_{2}}{x_{1}^{2}}\right)\left(\right.$ resp., $\left.u(x)=\left(-x_{1}\right)^{\mu} v\left(\frac{x_{2}}{x_{1}^{2}}\right)\right)$ defines a solution of 5.5$)$ in the half plane $\left\{x_{1}>0\right\}$ (resp. $\left.\left\{x_{1}<0\right\}\right)$.

Take a sufficient large open subset $\Omega$ of $\mathbb{R}$, Theorem B.1.7 shows that all hyperfunction solutions $v(y)$ of $Q v(y)=0$ are real analytic functions. That is to say, $u\left(x_{1}, x_{2}\right)=x_{1}^{\mu} v(y)=x_{1}^{\mu} v\left(\frac{x_{2}}{x_{1}^{2}}\right)$ is real analytic except the line $x_{1}=0$. Moreover, along the line $x_{1}=0$, the operator $P$ is elliptic if $x_{2}>0$, according to Theorem 5.2.1, $u$ is real analytic on the half line $\left\{x_{1}=0, x_{2}>0\right\}$. Hence

$$
\mathrm{WF}_{a}(u) \subset\left\{\left(\left(0, x_{2}, \xi_{1}, \xi_{2}\right) \in T^{*} \mathbb{R}^{2} \backslash 0 \mid x_{2} \leq 0, \xi_{1}^{2}+x_{2} \xi_{2}^{2}=0\right\}\right.
$$

We would like to discuss the behavior of the solution $u$ of $P u=0$ along the half line $\left\{x_{1}=0, x_{2} \leq 0\right\}$. It is equivalent to consider the behavior of the solution $v(y)$ of $Q v(y)=0$ at $-\infty$.

Regard $Q v(y)=0$ as an equation in the complex plane, it has three regular singular points, i.e., $\infty$ and $\frac{-1 \pm \sqrt{1-16 c}}{8}$. Then by affine transformation $Q v=0$ can 
be transformed into the standard Euler's hypergeometric equation 3

$$
y(1-y) v^{\prime \prime}(y)+[r-(p+q+1) y] v^{\prime}(y)-p q v(y)=0
$$

with three regular singular points at 0,1 and $\infty$. Here

$$
\begin{aligned}
p & =-\frac{\mu}{2}, \\
q & =\frac{1-\mu}{2} \\
r & =-\frac{1}{2}\left(1-\frac{1}{\sqrt{1-16 c}}\right) \mu+\frac{\theta}{\sqrt{1-16 c}}+\frac{3}{4}\left(1-\frac{1}{\sqrt{1-16 c}}\right) .
\end{aligned}
$$

As showed in [22], under certain conditions with the coefficients we can construct analytic solutions of the hypergeometric equation. The roots of indicial equation (as know as characteristic equation) of (5.8) at $\infty$ are $\frac{\mu}{2}$ and $\frac{\mu-1}{2}$. Near the singular point $\infty$, choose two linear independent solutions of (5.8) analytic in a neighborhood of the real axis and normalized so that

$$
v_{k}(y)=(-y)^{\frac{\mu-k}{2}} h_{k}\left(\frac{1}{y}\right), \quad k=0,1
$$

as $y \rightarrow-\infty$, where $h_{k}(z)$ is an analytic function near zero such that $h_{k}(0)=1$. Define

$$
u_{k}\left(x_{1}, x_{2}\right)= \begin{cases}x_{1}^{\mu} v_{k}\left(\frac{x_{2}}{x_{1}^{2}}\right), & x_{1}>0 \\ (-1)^{k}\left(-x_{1}\right)^{\mu} v_{k}\left(\frac{x_{2}}{x_{1}^{2}}\right), & x_{1}<0\end{cases}
$$

for $k=0,1$, then we obtain a solution of $P u=0$ in $x_{1} \neq 0$ can be written as linear composition of

$$
u_{k}\left(x_{1}, x_{2}\right)=x_{1}^{k}\left|x_{2}\right|^{\frac{\mu-k}{2}} h_{k}\left(\frac{x_{1}^{2}}{x_{2}}\right), \quad k=0,1,
$$

near the half line $\left\{x_{1}=0, x_{2}<0\right\}$. Since $u_{k}$ is real analytic along this half line, $u_{k}$ is in fact a solution of $P u=0$ in the complement of the half line $\left\{x_{1}=0, x_{2} \geq 0\right\}$.

Write

$$
u=t u_{0}+s u_{1}, \quad t, s \in \mathbb{R}
$$

since $u$ is real analytic in the half line $\left\{\left(0, x_{2}\right) \mid x_{2}>0\right\}, s$ must vanishes (otherwise $u$ is not real analytic there). That is, $u_{0}$ is a solution of $P u=0$ on $\mathbb{R}^{2} \backslash\{0\}$. Actually Lemma 5.2.2 shows that $u_{0}$ are hyperfunction solutions of $P u=0$ on $\mathbb{R}^{2}$ while provided some restrictions on $\mu$.

To discuss the solutions of the hypergeometric equation $(5.8)$, we need certain conditions between the parameters $p, q$ and $r$. Guillemin and Schaeffer [22] had shown us an ample supply of solutions of (5.5) with suitable restrictions on those parameters

\footnotetext{
${ }^{3}$ See Appendix E.
} 
$p, q$ and $r$. In fact it is sufficient that we have $c-a$ is an integer, where

$$
\begin{aligned}
c-a & =\frac{\mu}{2 \sqrt{1-16 c}}+\frac{\theta}{\sqrt{1-16 c}}+\frac{3}{4}\left(1-\frac{1}{\sqrt{1-16 c}}\right) \\
& =\frac{2 \mu+4 \theta-3}{4 \sqrt{1-16 c}}+\frac{3}{4} .
\end{aligned}
$$

There are infinite many $\mu^{\prime} s$ to make $c-a$ to be integer.

Choose suitable $\mu$ and let $u \in \mathscr{B}\left(\mathbb{R}^{2}\right)$ be an associated solution of $L u=0$, then

$$
\left.\mathrm{WF}_{a}(u) \subset\left\{\left(0,0,0, \xi_{2}\right)\right) \in T^{*} \mathbb{R}^{2} \backslash 0\right\}
$$

For a general operator $P$, let $F$ be a microdifferential operator induced a quantized contact transformation between $P$ and $L$, such that $P F-F L=E$, and let $v=F u$. Then $P v=E u$. (5.4b) implies $\mathrm{WF}_{a}(E u)$ is empty, so $E u \in \mathcal{A}(X)$. Finally, if $N_{2}$ does not contain $(0,0,0,-1)$, then by $5.4 \mathrm{a})$

$$
\mathrm{WF}_{a}(v) \subset\left\{\psi(z): z \in \mathrm{WF}_{a}(u) \text { and } z \in N_{2}\right\}
$$

consists of the single ray.

\subsection{Hyperbolic attracting case}

Theorem 5.3.1. Let $P$ be a microdifferential operator satisfies conditions in Lemma 5.1.3 then near an hyperbolic attracting/repelling radial point, there exist a hyperfunction solution $u$ of the equation $P u=f \in \mathcal{A}(M)$ such that $u$ has minimal analytic singularity in radial direction.

Proof. Near the hyperbolic attracting (resp. repelling) radial point, we have a normal form $P_{N h}$ of $P$ :

$$
P_{N h}=b x_{1} \partial_{x_{1}}+x_{2} \partial_{x_{2}}+\theta, \quad 0<b<1 .
$$

The generic radial point of $P$ is isolated implies $P$ is microlocally of real principal type near radial point $\nu^{0}$, with the direction $\mathbb{R}_{+} \nu^{0}$ removed.

The principal symbol of $P_{N h}$ is

$$
\sigma^{1}\left(P_{N h}\right)=\sqrt{-1}\left(b x_{1} \xi_{1}+x_{2} \xi_{2}\right) .
$$

Since $\partial_{x_{2}}$ is elliptic near the radial point, we obtain the operator

$$
P_{N h}=b x_{1} \partial_{x_{1}}+x_{2} \partial_{x_{2}}+\theta, \quad 0<b<1 .
$$

from

$$
b x_{1} \partial_{x_{1}} \partial_{x_{2}}+x_{2} \partial_{x_{2}}^{2}+\theta \partial_{x_{2}}, \quad 0<b<1
$$


which indicates $\xi_{2} \neq 0$, and the characteristics

$$
\operatorname{Char}\left(P_{N h}\right)=\left\{\left(x_{1}, x_{2}, \xi_{1}, \xi_{2}\right) \in T^{*} \mathbb{R}^{2} \backslash 0 \mid b x_{1} \xi_{1}+x_{2} \xi_{2}=0\right\} .
$$

The radial point $\nu^{0}=\left(0,0,0, \xi_{2}\right)\left(\xi_{2}>0\right)$ is microlocally isolated implies that

$$
\nabla_{\xi} \sigma^{1}\left(P_{N h}\right) \neq 0 \quad \text { for } \quad\left(x_{1}, x_{2}, \xi_{1}, \xi_{2}\right) \in \operatorname{Char}(P) \backslash\left\{\mathbb{R}_{+} \nu^{0}\right\}
$$

Consider the Fourier transformation of the equation $L u=f$ on both sides, we have $\widehat{L u}=\hat{f}$, where

$$
\widehat{L u}=\left(-b \xi_{1} \frac{\partial}{\partial \xi_{1}}-\xi_{2} \frac{\partial}{\partial \xi_{2}}+(\theta-\lambda-1)\right) \hat{u}
$$

Denote that

$$
-\hat{L}=b \xi_{1} \frac{\partial}{\partial \xi_{1}}+\xi_{2} \frac{\partial}{\partial \xi_{2}}+\beta
$$

Here, $\beta$ is a constant of value $-\theta+b+1$.

At first fix a constant $b \in(0,1)$, let $u \in \mathscr{Q}\left(\mathbb{D}^{2}\right)$ be a slowly increasing hyperfunction (which has been introduced by Sato [65] under the name of Fourier hyperfunctions) whose Fourier transformation is well-defined as a slowly increasing hyperfunction, which is given by the function

$$
\hat{u}\left(\xi_{1}, \xi_{2}\right)=\left(\xi_{2}\right)^{-\beta} \phi\left(\frac{\xi_{1}}{\xi_{2}^{b}}\right) \chi\left(\xi_{2}\right)
$$

here $\phi \in \mathcal{A}_{*}$ is a rapidly decreasing real analytic function, i.e., $\phi(t)=e^{-\left(1+t^{b^{\prime}}\right)}$ with a enough large even integer $b^{\prime}$ satisfying $b^{\prime}(1-b)>1$ and $\chi \in \overline{\mathcal{A}}$ is a slowly increasing real analytic function, i.e., $\chi\left(\xi_{2}\right)=-e^{-\left(\xi_{2}^{2}+1\right)}+1$. Moreover, we have

$$
\hat{f}\left(\xi_{1}, \xi_{2}\right)=\left(\xi_{2}\right)^{-\beta} \phi\left(\frac{\xi_{1}}{\xi_{2}^{b}}\right) \chi^{\prime}\left(\xi_{2}\right)
$$

Notice that $\xi_{2} \neq 0$ implies $\hat{f}$ is an rapidly decreasing real analytic function, Proposition C.3.7 shows $f$ is real analytic.

By computation $\operatorname{supp}_{\infty} \hat{u}$ is contained in proper cones containing the directions of $(0, \pm 1)$, so that

$$
\mathrm{WF}_{a}(u) \subset\left\{\left(x_{1}, x_{2}, 0, \xi_{2}\right): \xi_{2} \neq 0\right\} .
$$

However, we have $L u \in \mathcal{A}\left(\mathbb{R}^{2}\right)$ and

$$
\mathrm{WF}_{a}(u) \subset \operatorname{Char}(L) \cup \mathrm{WF}_{a}(f),
$$

then

$$
\mathrm{WF}_{a}(u) \subset\left\{\left(x_{1}, 0,0, \xi_{2}\right): \xi_{2} \neq 0\right\},
$$

as the principal symbol of $L$ vanishes on $\mathrm{WF}_{a}(u)$. 
We have

$$
\begin{aligned}
u\left(x_{1}, x_{2}\right) & =\int_{\mathbb{R}^{2}} \hat{u}\left(\xi_{1}, \xi_{2}\right) e^{\sqrt{-1}\left(x_{1} \xi_{1}+x_{2} \xi_{2}\right)} d \xi_{1} d \xi_{2} \\
& =\int_{-\infty}^{\infty} d \xi_{2}\left(\xi_{2}^{b-\beta} \chi\left(\xi_{2}\right) e^{\sqrt{-1} x_{2} \xi_{2}} \int_{-\infty}^{\infty} \phi\left(\frac{\xi_{1}}{\xi_{2}^{b}}\right) e^{\sqrt{-1} \frac{\xi_{1}}{\xi_{2}^{b}} \cdot \xi_{2}^{b} x} d\left(\frac{\xi_{1}}{\xi_{2}^{b}}\right)\right) \\
& =\int_{-\infty}^{\infty} \xi_{2}^{b-\beta} \chi\left(\xi_{2}\right) \check{\phi}\left(\xi_{2}^{b} x_{1}\right) e^{\sqrt{-1} x_{2} \xi_{2}} d \xi_{2},
\end{aligned}
$$

where $\check{\phi}$ is the one dimensional inverse Fourier transform of $\phi$. According to Proposition C.3.7. $\check{\phi}$ is exponentially decreasing, for any positive $\varepsilon$, the above integral is analytic for $x_{1}$ restricted to the set $\left\{\left|x_{1}\right| \geq \varepsilon\right\}$. Thus $u\left(x_{1}, x_{2}\right)$ is real analytic on $\left\{x_{1} \neq 0\right\}$. Moreover, the formula in the integral does not decreasing exponentially when $\left\{x_{1}=0\right\}$, then

$$
\mathrm{WF}_{a}(u)=\left\{\left(0,0,0, \xi_{2}\right): \xi_{2} \neq 0\right\}
$$

In this case, we take suitable covering and write $u$ as sum of boundary values of holomorphic functions. Then it is easy to find another solution $u^{\prime}\left(x_{1}, x_{2}\right)$ such that

$$
\mathrm{WF}_{a}\left(u^{\prime}\right)=\left\{\left(0,0,0, \xi_{2}\right): \xi_{2}>0\right\}
$$

For instance, substitute $\xi_{2}$ with $\xi_{2}+\sqrt{-1} 0$ in our assumption of $\widehat{u}$.

Remark 5.3.2. Let $n$ be the dimension of $X$. The characteristic variety of any $\mathcal{D}_{X}$-module is involutive. A coherent $D_{X}$-module $\mathscr{M}$ is said to be holonomic (resp. subholonomic) if the dimension of $\operatorname{supp}(\mathscr{M})$ equals $n$ (resp. $n+1)$.

There are plenty of results on holonomic systems and holonomic $\mathcal{D}$-modules, one can check Kashiwara [35], [KKK] [40] and [SKK] [63] for more information. However, if the equations (systems) are not holonomic, very few literatures can be found. For our operator $P_{N}$, the associated $\mathcal{D}$-module is not holonomic, and only in two dimensional case it is subholonomic. Actually, for $n=2$, we have

$$
\operatorname{dim}(\operatorname{supp}(\operatorname{Char}(\mathscr{M})))=3,
$$

then the associated $\mathcal{D}$-module $\mathscr{M} \simeq \mathcal{D} / \mathcal{D} P_{N}$ is subholonomic. There are a few results on subholonomic $\mathcal{D}$-module, i.e. [35], but here we do not plan to go further in the direction.

Here is an interesting example involving real analytic functions of positive type for our hyperbolic attractor case.

Example 5.3.3. For the normal form $P_{N h}$ with special constant coefficient $b$, one can construct hyperfunction solutions that the radial direction is the only singularity for each case.

Consider the function in Example 2.2.11 in two dimensional case, set $\varrho=-\alpha$, in 
the special case of $b=\frac{1}{2}$, we know that

$$
u\left(x_{1}, x_{2}\right)=\left(\sqrt{-1} x_{1}^{2}+x_{2}+\sqrt{-1} 0\right)^{\varrho}
$$

is a hyperfunction solution of $P_{N h} u=0$ with single singularity. More specifically, it is easy to check

$$
\left(\frac{1}{2} x_{1} \frac{\partial}{\partial x_{1}}+x_{2} \frac{\partial}{\partial x_{2}}+\alpha\right)\left(\sqrt{-1} x_{1}^{2}+x_{2}+\sqrt{-1} 0\right)^{-\alpha}=0 .
$$

Furthermore we have

$$
\text { S. S. }(u)=\left\{\left(\left(x_{1}, x_{2}\right), \sqrt{-1}\left(\xi_{1}, \xi_{2}\right) d x \infty\right) \mid x_{1}=x_{2}=\xi_{1}=0, \xi_{2}=1\right\} \in S^{*} \mathbb{R}^{2} .
$$

Moreover, in the case $0<b=\frac{p}{q}<1, p, q \in \mathbb{N}$, where $\frac{p}{q}$ is an irreducible fraction and $q$ is even, the hyperfunctions

$$
u\left(x_{1}, x_{2}\right)=\left(\sqrt{-1} t x_{1}^{\frac{q}{p}}+x_{2}+\sqrt{-1} 0\right)^{-\alpha}, t>0, k \in \mathbb{Z},-\alpha \neq 0,1,2 \cdots .
$$

are solutions of $L u=0$ with a single singularity $((0,0), \sqrt{-1}(0,1) d x \infty)$.

This can be generalized to higher dimensional cases by considering these hyperfunctions

$$
u\left(x_{1}, \cdots, x_{n}\right)=\left(\sqrt{-1} \sum_{i=1}^{n-1} x_{i}^{2}+x_{n}+\sqrt{-1} 0\right)^{\varrho}, \varrho \neq 0,1,2, \cdots
$$

which is a hyperfunction solution of $L u=0$, where

$$
L=\frac{1}{2}\left(x_{1} \frac{\partial}{\partial x_{1}}+\cdots+x_{n-1} \frac{\partial}{\partial x_{n-1}}\right)+x_{n} \frac{\partial}{\partial x_{n}}+(-\varrho) .
$$

Furthermore, $u$ has single singularity, i.e.,

$$
\text { S. S. }(u)=\{(x, \sqrt{-1} \xi d x \infty) \mid x=0, \xi=(0,0, \cdots, 1)\} .
$$

\subsection{Hyperbolic saddle case}

Near hyperbolic saddle radial point $\nu_{0}$ the operator $P$ has the normal form

$$
L=x_{1} \partial_{x_{1}}-\lambda x_{2} \partial_{x_{2}}+\theta, \quad \lambda>0 .
$$

By microlocal arrangement, for the equation $P u=f$ with $f \in \mathcal{A}\left(\mathbb{R}^{n}\right)$, one can equivalently consider the equation $L v=0$ with $\mathrm{WF}_{a}(v) \subset\left\{\left(x_{1}, x_{2}, \xi_{1}, \xi_{2}\right) \mid \xi_{2}>0\right\}$.

In section 4.4, we have found there are precisely four projected null bicharacteristics in the plane $\{\eta=1\}$ that go asymptotically to $(0,0,0,1)$, namely 


$$
\begin{aligned}
& \Sigma_{1}: x_{1}=e^{t}, x_{2}=\xi_{1}=0, \xi_{2}=1, \quad \text { as } t \rightarrow-\infty, \\
& \Sigma_{2}: x_{1}=-e^{t}, x_{2}=\xi_{1}=0, \xi_{2}=1, \quad \text { as } \quad t \rightarrow-\infty, \\
& \Sigma_{3}: x_{1}=x_{2}=0, \xi_{1}=e^{-(1+\lambda) t}, \xi_{2}=1, \quad \text { as } t \rightarrow+\infty, \\
& \Sigma_{4}: x_{1}=x_{2}=0, \xi_{1}=-e^{-(1+\lambda) t}, \xi_{2}=1, \quad \text { as } t \rightarrow+\infty .
\end{aligned}
$$

According to Remark 4.3.7, generally, we consider a hyperfunction solution of $L u=0$ in the half space $\{x>0\}$ of the following form

$$
u\left(x_{1}, x_{2}\right)=x_{1}^{-\theta} v\left(x_{1}^{\lambda} x_{2}\right)
$$

where $v \in \mathscr{B}(\mathbb{R})$.

Definition 5.4.1. A hyperfunction $f(x)$ is said to contain $x_{1}$ as a real analytic parameter in a neighborhood of $x$ if $\left(x, \pm \sqrt{-1} d x_{1} \infty\right) \notin$ S.S. $(f)$. If there is no danger of confusion, say $f$ is real analytic in $x_{1}$ at $x$.

Proposition 5.4.2. Let $P$ be a pseudo-differential operator with real analytic coefficients of order $m$, and $P$ is of real principle type, i.e., on the characteristic set $\left\{(x, \xi): p_{m}(x, \xi)=0\right\}$ of $P$, one has $\nabla_{\xi} p_{m}(x, \xi) \neq 0$. Let $u$ be solution of $P u=f \in \mathcal{A}$. If $\left(x_{0}, \xi_{0}\right)$ satisfies $p_{m}\left(x_{0}, \xi_{0}\right)$ and $\left(x_{0}, \xi_{0}\right) \notin \mathrm{WF}_{a}(u)$, then the bicharacteristic $\gamma$ pass through $\left(x_{0}, \xi_{0}\right)$ satisfies that $\gamma \cap \mathrm{WF}_{a}(u)=\emptyset$.

The generic radial point is isolated implies: microlocally the operator $P$ is of real principal type near the radial point, with the radial direction removed.

If $u$ is a solution of $L u=0$, each of the four projected null bicharacteristics must either be entirely contained in $\mathrm{WF}_{a}(u)$ or else be disjoint from $\mathrm{WF}_{a}(u)$.

Theorem 5.4.3. Let $P$ be a microdifferential operator satisfies the conditions in Lemma 5.1.3 Near an hyperbolic saddle radial point, for any hyperfunction solution $u$ of the equation $P_{N h} u=f \in \mathcal{A}(M)$, if $\left\{\left(0,0,0, \xi_{2}\right) \mid \xi_{2}>0\right\} \subset \mathrm{WF}_{a}(u)$, then at least one of the four projected null bicharacteristics $\Sigma_{i}, i=1,2,3,4$ must be contained in $\mathrm{WF}_{a}(u)$. That is, if all of the four curves do not intersect the singular spectrum of the solution $u$, then $(0,0,0,1)$ is not in $\mathrm{WF}_{a}(u)$. Moreover, if one of $\Sigma_{3}$ and $\Sigma_{4}$ is contained in $\mathrm{WF}_{a}(u)$, so is the other.

Proof. We will prove it by contradiction. Assume none of four projected null bicharacteristics intersect with $\mathrm{WF}_{a}(u)$. Taking a suitable conic neighborhood of the radial direction $\left\{\left(0,0,0, \xi_{2}\right), \xi_{2}>0\right\}$, and by assumption that $\Sigma_{1}$ does not meet $\mathrm{WF}_{a}(u)$, we have

$$
\left\{\left(x_{1}, 0,0, \pm 1\right): x_{1}>0\right\} \nsubseteq \mathrm{WF}_{a}(u),
$$

that is $u$ contain $x_{2}$ as a real analytic parameter in a neighborhood of $\left\{\left(x_{1}, 0\right) \mid x_{1}>0\right\}$. Moreover, since $\varphi:\left(x_{1}, x_{2}\right) \mapsto\left(x_{1}^{\prime}, x_{2}^{\prime}\right)=\left(x_{1}, x_{1}^{\lambda} x_{2}\right)$ is a real analytic coordinate transformation in the half space $\left\{x_{1}>0\right\}$, then

$$
\varphi^{*}\left(\mathrm{WF}_{a}(u)\right)=\mathrm{WF}_{a}\left(\varphi^{*} u\right)
$$


Hence $u$ is real analytic in $x_{2}^{\prime}$ near $\left\{\left(x_{1}, 0\right): x_{1}>0\right\}$. That is to say $v$ is a real analytic function near zero. Then

$$
u\left(x_{1}, x_{2}\right)=x_{1}^{-\theta} v\left(x_{1}^{\lambda} x_{2}\right)
$$

defines a hyperfunction $u_{+}$supported on $x_{1} \geq 0$ such that $u-u_{+}=0$ on the half space $\left\{x_{1}>0\right\}$, i.e. $u_{+}\left(x_{1}, x_{2}\right)=x_{1+}^{-\theta} v_{+}\left(x_{1+}^{\lambda} x_{2}\right)$. Similarly, near $\left\{\left(x_{1}, 0\right): x_{1}<0\right\}$, one can find a hyperfunction $u_{-} \in \mathscr{B}\left(\mathbb{R}^{2}\right)$ supported on $\left\{x_{1} \leq 0\right\}$ such that $u-u_{-}=0$ on $\left\{x_{1}<0\right\}$.

Consider a decomposition $u=u_{+}+u_{-}+u_{0}$, where the hyperfunctions $u_{+}, u_{-}$and $u_{0}$ are supported on the set $\left\{x_{1} \geq 0\right\},\left\{x_{1} \leq 0\right\}$ and $\left\{x_{1}=0\right\}$ respectively. Moreover, we have $\mathrm{WF}_{a}\left(u_{+}\right) \subset\left\{x_{1}=0\right\}, \mathrm{WF}_{a}\left(u_{-}\right) \subset\left\{x_{1}=0\right\}$ and $\mathrm{WF}_{a}\left(u_{0}\right) \subset\left\{x_{1}=0\right\}$.

Now we want to show the radial direction $\left\{\left(0,0,0, \xi_{2}\right), \xi_{2}>0\right\}$ does not belong to the singular spectrum of any of the above terms.

According to our discussion, $u(x)$ contains $x_{2}$ as a real analytic parameter in a neighborhood of $\left\{\left(x_{1}, 0\right) \mid x_{1} \neq 0\right\}$. From the above construction we have

$$
\left\{\left(0,0,0, \xi_{2}\right) \mid \xi_{2}>0\right\} \nsubseteq \mathrm{WF}_{a}\left(u_{+}\right),
$$

and

$$
\left\{\left(0,0,0, \xi_{2}\right) \mid \xi_{2}>0\right\} \nsubseteq \mathrm{WF}_{a}\left(u_{-}\right) .
$$

Moreover, $\mathrm{WF}_{a}(u) \cap \Sigma_{k}=\emptyset$ for $k=3$ and 4 . That is to say $u$ contain $x_{1}$ and $x_{2}$ as real analytic parameters near $(0,0)$, and hence

$$
\left\{\left(0,0,0, \xi_{2}\right) \mid \xi_{2}>0\right\} \nsubseteq \mathrm{WF}_{a}\left(u_{0}\right),
$$

contradiction. 


\section{Chapter 6}

\section{Analytic singularities in higher dimensions}

In Chapter 5 we have studied the analytic singularities for two dimensional case completely, the higher dimensional cases are much more complicate. Let us have a look at three dimensional case first, take local coordinates $x=\left(x^{\prime}, x_{3}\right)=\left(x_{1}, x_{2}, x_{3}\right)$, at first the normal form can be written as

$$
P_{N}=\left\langle A D_{x^{\prime}}, D_{x^{\prime}}\right\rangle+\left\langle B x^{\prime}, D_{x^{\prime}}\right\rangle D_{x_{3}}+\left\langle C x^{\prime}, x^{\prime}\right\rangle D_{x_{3}}^{2}+\gamma x_{3} D_{x_{3}}^{2}+\theta D_{x_{3}} .
$$

Here $A, B, C$ are constant $2 \times 2$ matrices, $A$ and $C$ are symmetric, $\gamma \in \mathbb{R} \backslash\{0\}$ and $\theta \in \mathbb{C}$.

The radial point of $P_{N}$ is $(0,0,0 ; 0,0,1) \in S^{*} \mathbb{R}^{3}$.

The matrix (3.2) indicates that the linear part of the related contact vector field $V$ to $P_{N}$ in three dimensional case is of the form

$$
\left(\begin{array}{c|c}
\gamma & 0 \cdots 0 \\
\hline 0 & \\
\vdots & \frac{\gamma}{2} I+A \\
0 &
\end{array}\right)
$$

where $A$ is a $4 \times 4$ symplectic matrix of the the following form

$$
\left(\begin{array}{cc|cc}
-b_{11}+\frac{\gamma}{2} & -b_{12} & -2 c_{11} & -2 c_{12} \\
-b_{21} & -b_{22}+\frac{\gamma}{2} & -2 c_{21} & -2 c_{22} \\
\hline 2 a_{11} & 2 a_{12} & b_{11}-\frac{\gamma}{2} & b_{12} \\
2 a_{21} & 2 a_{22} & b_{21} & b_{22}-\frac{\gamma}{2}
\end{array}\right)
$$

with respect to the symplectic basis

$$
\frac{\partial}{\partial_{\xi_{1}}}, \frac{\partial}{\partial_{\xi_{2}}}, \frac{\partial}{\partial_{x_{1}}}, \frac{\partial}{\partial_{x_{2}}}
$$


According to the previous analysis in section 3.3, the eigenvalues of the symplectic matrix $A$ will be of the follow cases:

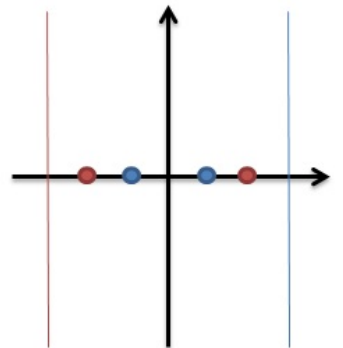

(a) Hyperbolic attracting/repelling

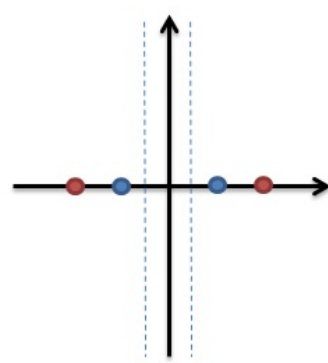

(c) Hyperbolic non-attracting/non-repelling

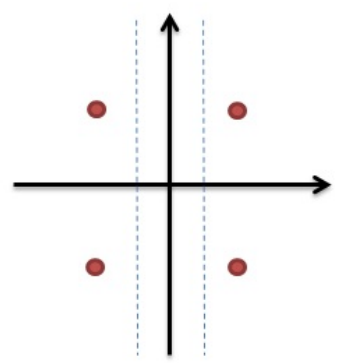

(e) Loxodromic non-attracting/non-repelling

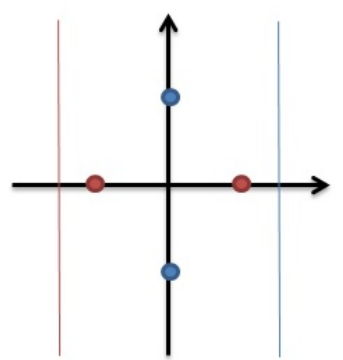

(g) Mixed type attracting/repelling

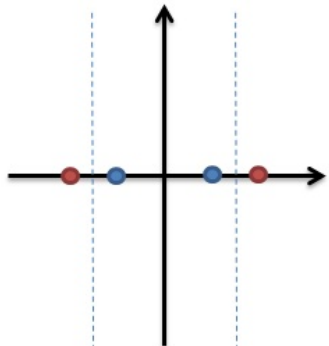

(b) Hyperbolic non-attracting/non-repelling

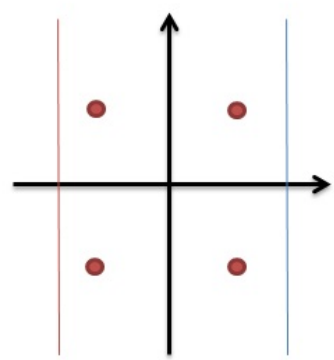

(d) Loxodromic attracting/repelling

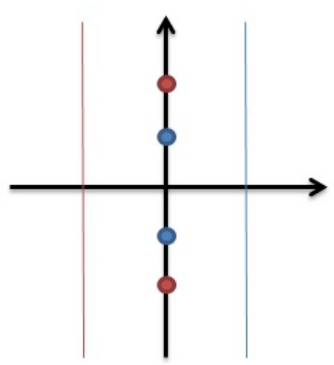

(f) Elliptic

attracting/repelling

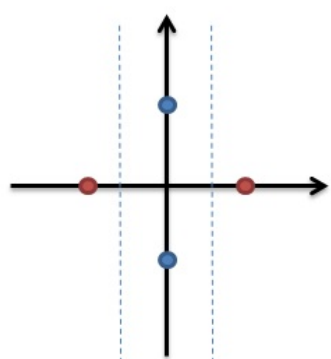

(h) Mixed type non-attracting/non-repelling

Figure 6-1: Classification of eigenvalues of $A$ for $3 D$ 
(i) 6-1a , 6-1b and (6-1c). A is hyperbolic and the eigenvalues are only of type (i), i.e., four real numbers, $\lambda_{1}, \lambda_{2}=-\lambda_{1}, \lambda_{3}, \lambda_{4}=-\lambda_{3}$.

(ii) (6-1d) and (6-1e). A is loxodromic and the eigenvalues are only of type (iii), i.e., four complex eigenvalues but not pure imaginary, $\lambda_{1}, \lambda_{2}=-\lambda_{1}, \lambda_{3}=\bar{\lambda}_{1}, \lambda_{4}=$ $-\bar{\lambda}_{1}$.

(iii) (6-1f). $\mathrm{A}$ is elliptic and the eigenvalues are only of type (ii), i.e., four pure imaginary eigenvalues, $\lambda_{1}, \lambda_{2}=\bar{\lambda}_{1}=-\lambda_{1}, \lambda_{3}, \lambda_{4}=\bar{\lambda}_{3}=-\lambda_{3}$.

(iv) (6-1g) and (6-1h). A is of mixed type and the eigenvalues are of mixed type (i) and type (iii), i.e., two real and two pure imaginary eigenvalues, $\lambda_{1}, \lambda_{2}=$ $-\lambda_{1}, \lambda_{3}, \lambda_{4}=\bar{\lambda}_{3}=-\lambda_{3}$. The radial point is neither hyperbolic nor elliptic, i.e., of mixed type.

More specifically, consider the relation between the four eigenvalues and the values of $\frac{\gamma}{2}$, we have eight cases as showed in Figure 6-1, the dots represent the possible eigenvalues of $A$, and the (dotted) vertical lines in the pictures represent the possible values of $\frac{\gamma}{2}$.

More specifically, in the four cases, $(6-1 \mathrm{a}),(\sqrt{6-1 \mathrm{~d}}),(6-1 \mathrm{f})$ and $(6-1 \mathrm{~g})$, the eigenvalues related to the linear part $\frac{\gamma}{2} I+A$ of the vector field are in common that, they convex hull of the four eigenvalues does not contain zero. That is, the eigenvalues are of Poincare type, and the eigenvalues of the left four cases $(6-1 \mathrm{~b}),(6-1 \mathrm{c}),(6-1 \mathrm{e})$ and $(6-1 \mathrm{~h})$ are of Siegel type. Actually, according to Definition 5.1.1, the radial point is attracting (or repelling) in four cases (6-1a), (6-1d), (6-1f) and (6-1g), and in the left four cases, the radial point is neither attracting nor repelling.

\subsection{Attracting case}

Theorem 6.1.1. Let $\left(x_{0}, \xi_{0}\right)$ be a generic radial point which is either attracting or repelling. Then there exist a hyperfunction $u$ such that $P u$ is real analytic and $\mathrm{WF}_{a}(u)$ just consists of the minimal singularity, i.e., the radial direction.

We will prove the theorem in three cases respectively, i.e., the radial point $\left(x_{0}, \xi_{0}\right)$ is elliptic, hyperbolic and of mixed type.

\subsubsection{Elliptic attracting case}

Take local coordinates $x=\left(y_{1}, \cdots, y_{n-1}, t\right)$, in the elliptic case, one has the normal form

$$
\left\{\sum_{i<n} \pm\left(-\frac{\partial^{2}}{\partial y_{i}^{2}}-c_{i} y_{i}^{2} \frac{\partial^{2}}{\partial t^{2}}\right)\right\}+\gamma t \frac{\partial^{2}}{\partial t^{2}}+\theta \frac{\partial}{\partial t}
$$

with $c_{i}>\frac{\gamma^{2}}{16}$ for $i=1,2, \cdots, n-1$. Here $x=(y, t)$ and denote the dual $\xi=(\eta, \tau)$. Let $F$ be the operator of partial Fourier transformation in the $t$ variable. Let $P^{F}=F P F^{-1}$, 
then

$$
P^{F}=\sum_{i<n} \pm\left(-\frac{\partial^{2}}{\partial y_{i}^{2}}+c_{i} y_{i}^{2} \tau^{2}\right)+\sqrt{-1} \gamma \tau^{2} \frac{\partial}{\partial \tau}+\sqrt{-1}(\theta+2) \tau .
$$

We will look for solutions of the equation $P^{F} u=0$ where

$$
u(\tau, y)=f(\tau, y \sqrt{\tau})
$$

and $f(\tau, y)$ is zero for $\tau<0$, is homogeneous of degree $\alpha$ in $\tau$ for $\tau>0$ and for fixed $\tau>0$ is an exponential decay real analytic function in $y$. Plugging into one has

$$
\begin{aligned}
P^{F} f(\tau, y \sqrt{\tau}) & =\tau\left\{\sum_{i<n} \pm\left(-\frac{\partial^{2} f}{\partial y_{i}^{2}}+c_{i} y_{i}^{2} f\right)+\frac{\sqrt{-1}}{2} \gamma \sum_{i<n} y_{i} \frac{\partial f}{\partial y_{i}}\right. \\
& +\sqrt{-1}(\theta+\gamma \alpha+2) f\}(\tau, y \sqrt{\tau}) .
\end{aligned}
$$

Taking $f=e^{\sqrt{-1} Q(y)} g$ where $Q(y)$ is the quadratic form

$$
Q(y)=\frac{\gamma}{8}\left( \pm y_{1}^{2} \pm y_{2}^{2} \pm \cdots \pm y_{n-1}^{2}\right),
$$

take suitable sign of $y_{i}$ such that $P^{F} u=0$ is equivalent to

$$
P^{F} f=e^{\sqrt{-1} Q(y)}\left(\sum_{i<n} \pm\left(-\frac{\partial^{2} g}{\partial y_{i}^{2}}+\left(c_{i}-\frac{\gamma^{2}}{16}\right) y_{i}^{2} g\right)+\sqrt{-1} \theta^{\prime} g\right)=0
$$

where $\theta^{\prime}=\theta+\gamma \alpha+\operatorname{sgn} \frac{Q}{4}-2$.

Since $c_{i}>\frac{\gamma^{2}}{16}$, the operator on the left hand side is the Schrödinger operator for a string of uncoupled harmonic oscillators. Its eigenfunctions are products of Hermite functions 1 and hence lie in $\mathcal{A}_{*}\left(\mathbb{R}^{n-1}\right)$.

Therefore for a countable number of $\theta^{\prime}$ ( $\theta^{\prime}$ totally imaginary) there exists a exponential decreasing real analytic function $h(y)$ such that such replacement of $g$ with $h$ is satisfied.

Define

$$
f(\tau, y)=e^{i Q(y)} \tau_{+}^{\alpha} h(y)
$$

where $\alpha$ is determined by $\theta^{\prime}$.

Go back to $P_{N e} u=0$, by construction,

$$
u(x)=\int f(\tau, y \sqrt{\tau}) e^{i t \tau} d \tau
$$

is a well-defined hyperfunction solution.

\footnotetext{
${ }^{1}$ See $\mathrm{F}$
} 
Lemma 6.1.2. We have

$$
\text { S. S. } u(x)=((0,0), \sqrt{-1}(0, \tau) d x \infty) .
$$

Proof. Notice that $u(x)=u(y, t)$ contained $y$ as a real analytic parameter, then

$$
\text { S. S. }(u) \subset\{(y, t, \sqrt{-1}(0, \tau) d x \infty)\} .
$$

Moreover, S. S. $(u)$ is contained in $\operatorname{Char}\left(P_{N e}\right)$, we have

$$
\left\{\sum_{i<n} \pm\left(\eta_{i}^{2}+c_{i} y_{i}^{2} \tau^{2}\right)\right\}+\gamma t \tau=0
$$

We have $h(y \sqrt{t})$ is exponential decreasing in $y$ (resp., $t$ ) for $t \neq 0$ (resp., $y \neq 0$ ). That is for $y \neq 0, \tau>0, f(\tau, y \sqrt{\tau})$ is in $\mathcal{A}_{*}$ for both parameter $y$ and $\tau$. According to Proposition C.3.7, the partial Fourier transformation acting on $t$ is an isomorphism of $\mathcal{A}_{*}, u(y, t)$ is exponential decreasing for $y \neq 0$ and $t \neq 0$. And for $(y, t)=(0, t)$, we have $u(y, t)$ is not real analytic at $(0,0)$.

That is to say

$$
\text { S. S. }(u(y, t))=\{(0,0), \sqrt{-1}(0, \tau) d x \infty\} \text {. }
$$

\subsubsection{Hyperbolic attracting case}

Consider the normal form

$$
P=P_{N h}=\sum_{i, j<n} b_{i j} y_{i} \frac{\partial}{\partial y_{j}}+t \frac{\partial}{\partial t}+\theta,
$$

which has a radial point at $\left(0 ; 0^{\prime}, 1\right) \in S^{*} \mathbb{R}^{n}$. Let $B=\left(b_{i j}\right)$ and $\lambda_{1}, \cdots, \lambda_{n-1}$ be the eigenvalues of $B$. Assume all the eigenvalues are real and we can write the normal form as

$$
P_{N h}=\sum_{i<n} \lambda_{i} y_{i} \frac{\partial}{\partial y_{i}}+t \frac{\partial}{\partial t}+\theta
$$

Lemma 6.1.3. The radial point $\left(0 ; 0^{\prime}, 1\right)$ is attracting (resp. repelling) if and only if

$$
0<\operatorname{Re} \lambda_{k}<1, \quad \text { for any } k=1, \cdots, n-1 \text {. }
$$

Let $F$ be the Fourier transformation and let $P^{F}=F P F^{-1}$, i.e.,

$$
P^{F}=\sum \lambda_{i} \eta_{i} \frac{\partial}{\partial \eta_{i}}+\tau \frac{\partial}{\partial \tau}+\theta^{\prime}
$$

where $\theta^{\prime}=\operatorname{trace} B+\theta+1$. 
Theorem 6.1.4. (Minimal singularities) In $n$ dimensional hyperbolic attracting case, near a generic radial point there is a hyperfunction solution $v$ of the equation

$$
P v=0
$$

contains the radial direction as the only analytic singularity.

Proof. Under the above discussion, we can reduce the initial problem

$$
P^{F} f=0, \quad f(1, \eta)=f_{0}(\eta)
$$

to the form

$$
\tau \frac{\partial}{\partial \tau} f+\sum_{i<n} \lambda_{i} \eta_{i} \frac{\partial}{\partial \eta_{i}} f=k f, \quad f(1, \eta)=f_{0}(\eta)
$$

where $0<\lambda_{i}<1$ and $f_{0}(\eta) \in \mathcal{A}_{*}\left(\mathbb{R}^{n-1}\right)$ is an exponential decay real analytic function. For instance, assume $\max \left\{\lambda_{i}\right\}=\lambda_{0}$, and take a large enough even integer $\lambda_{0}^{\prime}$ satisfying $\lambda_{0}^{\prime}\left(1-\lambda_{0}\right)>1$, and we can set $f_{0}(\eta)=e^{-\left(1+\eta^{\lambda_{0}^{\prime}}\right)}$.

Consider the integral curve of the vector field $\tau \frac{\partial}{\partial \tau}+\sum_{i<n} \lambda_{i} \eta_{i} \frac{\partial}{\partial \eta_{i}}$ with suitable parameters, we have the formula

$$
f\left(\tau, \eta_{1}, \cdots, \eta_{n-1}\right)=\tau^{k} f_{0}\left(\eta_{1} \tau^{-\lambda_{1}}, \cdots, \eta_{n-1} \tau^{-\lambda_{n-1}}\right)
$$

Let $\rho(\tau) \in \overline{\mathcal{A}}$ be a slowly increasing real analytic function, i.e. $e^{-\left(1+\tau^{2}\right)}+1$, and set

$$
g(\eta, \tau)=\rho(\tau) f(\tau, \eta)
$$

Let

$$
v(y, t)=\int g(\tau, \eta) e^{i(t \tau+y \eta)} d \tau d \eta,
$$

then $P v$ is the inverse Fourier transformation of $P^{F} g$. We have $P^{F} g \in \mathcal{A}_{*}$, so $P v$ is real analytic.

By computation $\operatorname{supp}_{\infty} \hat{v}$ is contained in proper cones containing the direction of $(0, \pm 1)$, so that

$$
\mathrm{WF}_{a}(v) \subset\{(y, t, 0, \tau): \tau \neq 0\} .
$$

However, we have $P u \in \mathcal{A}\left(\mathbb{R}^{n}\right)$ and

$$
\mathrm{WF}_{a}(v) \subset \operatorname{Char}(P) \cup \mathrm{WF}_{a}(f),
$$

then

$$
\mathrm{WF}_{a}(v) \subset\{(y, 0,0, \tau): \tau \neq 0\},
$$

as the principal symbol of $P$ must vanish on $\mathrm{WF}_{a}(v)$. 
We observe that

$$
\begin{aligned}
v(y, t) & =\int_{\mathbb{R}^{n}} g(\eta, \tau) e^{\sqrt{-1}(y \eta+t \tau)} d \eta d \tau \\
& =\int_{-\infty}^{\infty} d \tau\left(\tau^{k} \rho(\tau) e^{\sqrt{-1} t \tau} \int_{\mathbb{R}^{n-1}} f_{0}\left(\eta_{1} \tau^{-\lambda_{1}}, \cdots, \eta_{n-1} \tau^{-\lambda_{n-1}}\right) e^{\sqrt{-1} y \eta} d(\eta)\right) \\
& =\int_{-\infty}^{\infty} \tau^{k-\sum_{i=1} \lambda_{i}} \rho(\tau) \check{f}_{0}\left(\tau^{\lambda_{1}} y_{1}, \cdots, \tau^{\lambda_{n-1}} y_{n-1}\right) e^{\sqrt{-1} t \tau} d \tau,
\end{aligned}
$$

where $\check{f}_{0}$ is the inverse Fourier transform of $f_{0}$. According to Proposition C.3.7, $\check{\phi}$ is exponentially decreasing, for any positive $\varepsilon$, the above integral is analytic for $y$ restricted to the set $\{|y| \geq \varepsilon\}$. Thus $v(y, t)$ is real analytic on $\{y \neq 0\}$. Moreover, the formula in the integral does not decreasing exponentially when $\{y=0\}$, then

$$
\mathrm{WF}_{a}(v)=\{(0,0 ; 0, \tau) \mid \tau \neq 0\}
$$

In this case, we take suitable covering and write $v$ as sum of boundary values of holomorphic functions. Then it is easy to find another solution $v^{\prime}(y, t)$ such that

$$
\mathrm{WF}_{a}\left(v^{\prime}\right)=\{(0,0 ; 0, \tau) \mid \tau>0\}
$$

\subsubsection{Mixed type attracting case}

Break the normal form into two parts, one is elliptic and another is hyperbolic attracting, we have

$$
P=\sum_{i=1}^{n-r-1} \pm\left(-\frac{\partial^{2}}{\partial y_{i}^{2}}-y_{i}^{2} c_{i} \frac{\partial^{2}}{\partial t^{2}}\right)+\sum_{j, k=1}^{r} b_{j k} z_{j} \frac{\partial}{\partial z_{k}} \frac{\partial}{\partial t}+t \frac{\partial^{2}}{\partial t^{2}}+\theta \frac{\partial}{\partial t}
$$

where $c_{i}>\frac{1}{16}$. Here $x=(y, z, t)$ and its dual variables are $\xi=(\eta, \zeta, \tau)$. Assume $\lambda_{1}, \cdots, \lambda_{r}$ be the eigenvalues of the matrix $\left(b_{j k}\right)$, and satisfy the generical condition in Definition 4.2.4. Moreover, the attracting (resp. repelling) condition indicates that $\operatorname{Re} \lambda_{j} \in(0,1)$ for $1 \leq j \leq r$.

Assume the eigenvalues $\lambda_{i}$ 's are real and choose suitable coordinates of $z$ such that $b_{i j}=\lambda_{i} \delta_{i j}$. Let $F$ be the Fourier transformation. Then $p^{F}=F P F^{-1}$ has the following form

$$
\sum_{i} \pm\left(-c_{i} \tau^{2} \frac{\partial^{2}}{\partial \eta_{i}^{2}}+\eta_{i}^{2}\right)+\sqrt{-1} \tau\left(\sum_{j} \lambda_{j} \zeta_{j} \frac{\partial}{\partial \zeta_{j}}+\tau \frac{\partial}{\partial \tau}+\theta^{\prime}\right)
$$

where $\theta^{\prime}=\theta+2$. We look for a solution $v$ of $P^{F} v=0$ of the form

$$
v(\eta, \zeta, \tau)=\tau^{\alpha} f\left(\eta \tau^{-1 / 2}\right) g\left(\zeta_{1} \tau^{-\lambda_{1}}, \cdots, \zeta_{r} \tau^{-\lambda_{r}}\right),
$$


for $\tau>0$, where $f$ is a exponentially decay real analytic function in the $\eta$ variable and $g$ a exponentially decay function in the $\zeta$ variables. Plug (6.4) into 6.3), we have the formula

$$
\tau^{\alpha+1}\left(\sum \pm\left[-c_{i} \frac{\partial^{2} f}{\partial \eta_{i}^{2}}+\eta_{i}^{2} f \pm \frac{1}{2} \sqrt{-1} \eta_{i} \frac{\partial f}{\partial \eta_{i}}\right] g+\sqrt{-1}\left(\theta^{\prime}+\alpha\right) f g\right)
$$

takes value at $\left(\eta \tau^{-1 / 2}, \zeta_{1} \tau^{-\lambda_{1}}, \cdots, \zeta_{r} \tau^{-\lambda_{r}}, \tau\right)$.

Similar as the technique in section 6.1.1, we can transform the operator in square brackets to be Schrödinger operator for a string of uncoupled oscillators by taking

$$
Q(\eta)=\frac{1}{8} \sum_{i} \pm \eta_{i}^{2} .
$$

Then we can find a countable number of exponentially decay eigenfunctions $f \in \mathcal{A}_{*}$. Choose $f$ to be such an eigenfunction and choose $g$ to be a suitable exponentially decay real analytic function in $\zeta$ variables. Finally choose $\alpha$ to let $(6.5)$ vanishes identically.

Set

$$
\psi(\xi)=\psi(\eta, \zeta, \tau)=\rho(\tau) v(\eta, \zeta, \tau),
$$

where $\rho(\tau) \in \mathcal{A}_{*}(\mathbb{R})$. By choosing suitable $\rho(\tau)$ we can make sure that $\operatorname{supp}_{\infty} \hat{\psi}$ is contained in any open proper cone containing the direction of $\tau$ axis. Let

$$
u=\int \psi(\xi) e^{\sqrt{-1} x \xi} d \xi
$$

and then

$$
P u=\int\left(P^{F} \psi\right)(\xi) e^{\sqrt{-1} x \xi} d \xi
$$

The integrand in (6.6) is in the space of $\mathcal{A}_{*}$ so $P u$ is real analytic. By direct computation, we conclude that the analytic wave front set of $u$ has minimal singularities, which is concentrated in the radial direction, i.e., $\{(y, z, t ; \eta, \zeta, \tau)=(0,0,0 ; 0,0, \tau) \mid \tau>0\}$.

\subsection{Non-attracting case}

In this section, we are going to discuss the analytic singularities where the radial point is neither attractor nor repellent. In non-attracting cases, one can define, invariantly, two submanifolds of $S^{*} \mathbb{R}^{n}$, namely the attractor and the repellent submanifolds, which we call stable and unstable manifolds associated with the non-attractor radial point, and denote by $\Gamma_{1}$ and $\Gamma_{2}$ respectively.

In local coordinates the dimensions of stable and unstable manifolds are determined by the number of eigenvalues of the symplectic matrix $\frac{\gamma}{2} I+A$ with negative and positive real part, respectively. 
Suppose the stable and unstable manifolds has following form in local coordinates

$$
\Gamma_{1}=\left\{(x, 0) \mid x=\left(x_{1}, \cdots, x_{k_{1}}\right) \neq 0\right\}
$$

and

$$
\Gamma_{2}=\left\{(0, y) \mid y=\left(y_{k_{1}+1}, \cdots, y_{2 n-2}\right) \neq 0\right\}
$$

for some $k$.

Theorem 6.2.1. ([18]) Let $\Omega$ be a closed subset of $S^{*} \mathbb{R}^{n}$ so that $z_{0}=\{(0 ; 0,1)\} \in$ $S^{*} \mathbb{R}^{n}$ is not an isolated point in $\Omega$. If $\Omega$ is invariant under the projected bicharacteristics in an open neighborhood of $z_{0}$, then

$$
\Omega \cap \Gamma_{1} \neq \emptyset \quad \text { or } \quad \Omega \cap \Gamma_{2} \neq \emptyset
$$

Proof. If the behavior of the flow were described by

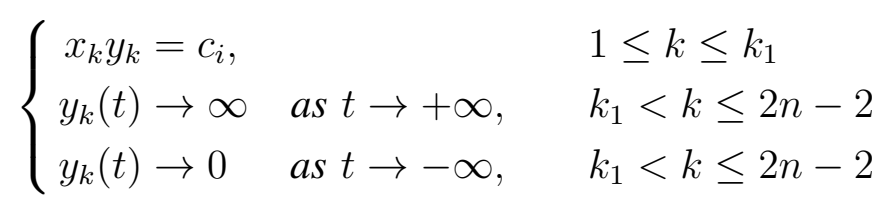

then we define

(i) $(x, y) \in$ Region I if $x_{k}>y_{k}$ for $1 \leq k \leq k_{1}$ and $y_{k}=0$ for $k>k_{1}$,

(ii) $(x, y) \in$ Region II if $(x, y) \notin$ Region I.

Consider a convergent sequence $\left\{t_{j}\right\}$ in a neighborhood of $z_{0}$ in $\Omega$, such that $t_{j} \rightarrow z_{0}$ as $k \rightarrow \infty$. Therefore there are at least one infinity subsequence $\left\{t_{k_{i}}\right\}$ belongs to Region I or Region II. Assume it belongs to Region II. Since $\Omega$ is invariant under the projected bicharacteristics in an open neighborhood of $z_{0}$, the flowing out of the subsequence $\left\{s_{k_{i}}\right\}$ is in the interior of Region II, we have $s_{k_{i}} \rightarrow s$ and $s \in \Sigma_{2} . \Omega$ is closed then $s \in \Omega$, that is $s \in \Omega \cap \Sigma_{2}$. Similarly we have $\Omega \cap \Sigma_{1} \neq \emptyset$ if $\left\{t_{k_{i}}\right\}$ belongs to Region I.

Remark 6.2.2. In two dimensional hyperbolic saddle case, the radial point is not an isolated point in the analytic wavefront set.

\subsubsection{Hyperbolic non-attracting case}

In this case, the real part of the eigenvalues of $A$ are non-zero. Suppose near the radial point we have the normal form

$$
L=\sum_{1 \leq i, j \leq n-1} b_{i j} x_{i} \frac{\partial}{\partial x_{j}}+x_{n} \frac{\partial}{\partial x_{n}}+\theta,
$$

and consider the equation $L u=f$ for $f \in \mathbb{R}^{n}$. 
Theorem 6.2.3. Let $u \in \mathscr{B}\left(\mathbb{R}^{n}\right)$ such that $(0 ; 0,1) \in \mathrm{WF}_{a}(u)$, then

$$
\mathrm{WF}_{a}(u) \cap \Gamma_{1} \neq \emptyset \quad \text { or } \quad \mathrm{WF}_{a}(u) \cap \Gamma_{2} \neq \emptyset \text {, }
$$

where $\Gamma_{1}$ and $\Gamma_{2}$ are the stable and unstable manifolds.

Here we only discuss the case that all the eigenvalues are real, the general case can be discussed later.

Assume

$$
L=\sum_{i=1}^{n-1} \lambda_{i} x_{i} \frac{\partial}{\partial x_{i}}+x_{n} \frac{\partial}{\partial x_{n}}+\theta,
$$

where $\lambda_{i} \in \mathbb{R}$ for $i=1, \cdots, n-1$ and $\theta \in \mathbb{C}$.

Moreover, we have the following generic assumption

(A1) The equation

$$
\sum_{i=1}^{n-1} t_{i} \lambda_{i}+t_{n}=\frac{t}{2}
$$

admits no integer solution $\left(t_{1}, \cdots, t_{n-1}, t_{n}\right) \in \mathbb{Z}^{n}$ with $t \neq 0$.

(A2) The non-attractor conditions says that there exists some $j_{k}, 0 \leq j_{k} \leq n-1$ such that $\lambda_{j_{k}}<0$ or $\lambda_{j_{k}}>1$. We have studied the analytic singularities of solution in the case $n=2$ in the previous section. Here we assume that $n \geq 3$ and suppose $j_{k} \geq 2$ by relabeling the variables. Moreover, we assume there is only one eigenvalue $\lambda_{j_{k}}$ satisfies the non-attractor condition.

By the Theorem 6.2.1, the aim Theorem 6.2.3 does not hold only if the radial point $\left(0 ; 0^{\prime}, 1\right)$ is an isolated point in the singular spectrum S. S. $(u)$.

Our strategy of the proof is the following:

(i) we first prove that we can assume that $u$ satisfies some regularity conditions,

(ii) secondly we prove that there exists a hyperfunction and a differential operator satisfies the assumptions of the theorem and similar conditions to (A1) and (A2) in $(n-1)$ dimensional case.

We use the induction method to prove it. The main idea is: assume in $n$ dimensional case, the radial direction is isolated in the analytic wavefront set for some solution, then one can find solution of $(n-1)$ dimensional case with the radial direction isolated in the analytic wavefront set. By induction, one can find solution to let the radial direction be isolated in two dimensional case near hyperbolic saddle radial point, contradiction.

Lemma 6.2.4. There exists an elliptic microdifferential operator $P$ near the radial point $\left(0 ; 0^{\prime}, 1\right)$ such that

$$
\begin{gathered}
u_{1}=P u, \\
\mathrm{WF}_{a}\left(u_{1}\right) \subset\left\{\left(0 ; 0^{\prime}, \xi_{n}\right) \mid \xi_{n}>0\right\}, \\
\hat{u}_{1}, \frac{\partial^{2}}{\partial \xi_{1}^{2}} \hat{u}_{1} \in \mathscr{Q}\left(\mathbb{D}^{n}\right) \cap L^{2}\left(\mathbb{R}^{n}\right) .
\end{gathered}
$$


and

$$
(L-N) u_{1} \in \mathcal{A}\left(\mathbb{R}^{n}\right)
$$

for some positive integer $N \cdot 2^{2}$

Proof. Since the radial direction is isolated in S.S. $(u)$, for elliptic $P$ near radial point, one has S. S. $\left(u_{1}\right)=$ S. S. $(u)$, we can choose suitable elliptic microdifferential operator $P$ cut off the conic neighborhood of the radial direction. Moreover, since the radial direction does not stay in $\mathrm{WF}_{a}(L u)$, in a conic neighborhood of the radial direction we have $(L-N) u_{1}$ is real analytic.

Taking $v_{0}=u_{1} * \overline{u_{1}^{\sharp}}$ and $v_{1}=\left(x_{1}^{2} u_{1}\right) * \overline{\left(x_{1}^{2} u_{1}\right)^{\sharp}}$, where $*$ is the convolution product and $w^{\sharp}(x)=w(-x)$ for $w \in \mathscr{B}\left(\mathbb{R}^{n}\right)$. The convolution products are well defined. We have the restriction of hyperfunctions

$$
w_{0}=\left.v_{0}\right|_{x_{1}=0} \quad \text { and } \quad w_{1}=\left.v_{1}\right|_{x_{1}=0} .
$$

The restrictions are well defined because of our hypothesis on $\mathrm{WF}_{a}\left(u_{1}\right)$.

Theorem 6.2.5. The following statements are equivalent:

(a) $\left\{\left(0 ; 0^{\prime}, \xi_{n}\right) \mid \xi_{n}>0\right\} \subset \mathrm{WF}_{a}\left(u_{1}\right)$,

(b) $\left\{\left(0 ; 0^{\prime}, \xi_{n}\right) \mid \xi_{n}>0\right\} \subset \mathrm{WF}_{a}\left(v_{0}\right)$,

(c) $\left\{\left(0^{\prime} ; 0^{\prime \prime}, \xi_{n}\right) \mid \xi_{n}>0\right\} \subset \mathrm{WF}_{a}\left(w_{0}\right)$ or $\left\{\left(0^{\prime} ; 0^{\prime \prime}, \xi_{n}\right) \mid \xi_{n}>0\right\} \subset \mathrm{WF}_{a}\left(w_{1}\right)$, where $0^{\prime}$ and $0^{\prime \prime}$ are the origin in $\mathbb{R}^{n-1}$ and in $\mathbb{R}^{n-2}$ respectively. Moreover,

$$
L_{k} w_{k} \in \mathcal{A}\left(\mathbb{R}^{n-1}\right), \quad k=0,1
$$

where

$$
L_{k}=\lambda_{2} x_{2} \frac{\partial}{\partial x_{2}}+\cdots+x_{n} \frac{\partial}{\partial x_{n}}+\theta_{k},
$$

and $\theta_{k}=-(2 N+1)-\sum_{j=2}^{n-1} \lambda_{j}+(-2 k+1) \lambda_{1}+2 \operatorname{Re} \theta$.

Proof. $\quad(\mathbf{a} \Longleftrightarrow \mathbf{b})$ From definition we have

$$
\hat{v}_{0}(\xi)=\left|\hat{u}_{1}(\xi)\right|^{2}, \quad \hat{v}_{1}(\xi)=\left|\frac{\partial^{2}}{\partial \xi_{1}^{2}} \hat{u}_{1}(\xi)\right|^{2}
$$

and $u_{1} \in \mathscr{Q}(\mathbb{D})$ and $\mathrm{WF}_{a}\left(u_{1}\right) \subset\left\{\left(0 ; 0^{\prime}, t\right) \mid t>0\right\}$, then

$$
\mathrm{WF}_{a}\left(\overline{u_{1}^{\sharp}}\right)=\left\{(-x, \xi) \mid(x, \xi) \in \mathrm{WF}_{a}(u)\right\},
$$

\footnotetext{
${ }^{2}$ Such shift of $N$ comes from the Fourier transformation, as one can see from the proof of Theorem 5.3.1 $L^{\prime}$ and $L$ are differential normal forms, $P L^{\prime}-L P=E$ for some elliptic operators $P$ and $E$, as we see from the end of section 5.1. and here $L^{\prime}$ is another normal form and which is actually a shift of $L$ by some positive integer $N$.
} 
and

$$
\mathrm{WF}_{a}\left(v_{0}\right) \subset\left\{(x+y ; \xi) \mid(x ; \xi) \in \mathrm{WF}_{a}\left(u_{1}\right),(y ; \xi) \in \mathrm{WF}_{a}\left(\overline{u_{1}^{\sharp}}\right)\right\} .
$$

(6.7) implies $\operatorname{supp}_{\infty} \hat{u}_{1}(\xi) \mid$ and $\operatorname{supp}_{\infty} \hat{v}_{0}(\xi)$ belong to the same conic neighborhood, by considering (6.8), (6.9) and Theorem C.5.8, our conclusion holds.

(c $\Longrightarrow \mathbf{b})$ Apply Definition 2.4.3 and Theorem 2.4.4 to our restriction, one can check that $M=\mathbb{R}^{n}, N=\mathbb{R}^{n-1}$ and S.S. $\left(v_{0}\right)=\left(0 ; 0^{\prime}, 1\right)$. To satisfy the condition S. S. $\left(v_{0}\right) \cap \sqrt{-1} S_{N}^{*} M=\emptyset$, we need

$$
\left\{\left(0, x^{\prime} ; \xi_{1}, 0^{\prime}\right) \mid \xi_{1} \neq 0\right\} \cap \mathrm{S} . \mathrm{S} .\left(v_{0}\right)=\emptyset,
$$

which is true under our hypothesis on the singular spectrum of $v_{0}$, the restriction is well defined.

That is, $w_{0}=\left.v_{0}\right|_{x_{1}=0}$ exists as an element of $\mathscr{B}\left(\mathbb{R}^{n-1}\right)$ and

$$
\mathrm{WF}_{a}\left(w_{0}\right) \subset\left\{\left(x^{\prime} ; \xi^{\prime}\right) \mid \exists \xi_{1}, \text { such that }\left(0, x^{\prime} ; \xi_{1}, \xi^{\prime}\right) \subset \mathrm{S} . \mathrm{S} .\left(v_{0}\right)\right\}
$$

for $v_{0} \in \mathscr{B}\left(\mathbb{R}^{n}\right)$.

We also can use FBI transformation to proof it directly.

$$
\begin{aligned}
\sup T_{h} v_{0}\left(x_{1}, x^{\prime}, \xi_{1}, \xi^{\prime}\right) & \geq \sup T_{h} v_{0}\left(0, x^{\prime}, 0, \xi^{\prime}\right) \\
& \gtrsim \sup T_{h} w_{0}\left(x^{\prime}, \xi^{\prime}\right),
\end{aligned}
$$

here $\gtrsim$ means the inequality is true up to some constant scaling.

$(\mathbf{a} \Longrightarrow \mathbf{c})$ We have

$$
\begin{aligned}
& \left|\hat{u}_{1}\left(t \xi_{1}, t \xi^{\prime}\right)\right| \\
& \leq\left|\int \frac{e^{-\sqrt{-1} x_{1} t \xi_{1}}}{1+x_{1}^{2}} \int e^{\sqrt{-1}\left\langle x^{\prime}, t \xi^{\prime}\right\rangle} u_{1}\left(x_{1}, x^{\prime}\right) d x^{\prime}\right| \\
& +\left|\int \frac{x_{1}^{2} e^{-\sqrt{-1} x_{1} t \xi_{1}}}{1+x_{1}^{2}} \int e^{\sqrt{-1}\left\langle x^{\prime}, t \xi^{\prime}\right\rangle} u_{1}\left(x_{1}, x^{\prime}\right) d x^{\prime}\right| \\
& \leq\left(\int \frac{d x_{1}}{\left(1+x_{1}^{2}\right)^{2}}\right)^{\frac{1}{2}}\left\{\left.\left.\left|\int\right| \hat{u}_{1}\left(\xi_{1}, t \xi_{2}, \cdots, t \xi_{n}\right)\right|^{2} d \xi_{1}\right|^{\frac{1}{2}}\right. \\
& \left.+\left.\left.\left|\int\right|\left(\frac{\partial^{2}}{\partial \xi_{1}^{2}} \hat{u}_{1}\right)\left(\xi_{1}, t \xi_{2}, \cdots, t \xi_{n}\right)\right|^{2} d \xi_{1}\right|^{\frac{1}{2}}\right\} .
\end{aligned}
$$

Then we have

$$
\left|\hat{u}_{1}(t \xi)\right| \leq C\left(\left|\hat{w}_{0}\left(t \xi^{\prime}\right)\right|^{\frac{1}{2}}+\left|\hat{w}_{1}\left(t \xi^{\prime}\right)\right|^{\frac{1}{2}}\right)
$$

for $t \gg 0$, some constant $C$ and for any $\xi^{\prime}$. That is, if $\operatorname{supp}_{\infty} \hat{u}_{1}(x, t \xi)$ is contained in a conic neighborhood of $(0,1)$, then at least one of $\operatorname{supp}_{\infty} \hat{w}_{i}\left(x^{\prime}, t \xi^{\prime}\right), i=0,1$ is contained in a conic neighborhood of $\left(0^{\prime}, 1\right)$. Apply Theorem C.5.8, we have finished to prove this step. 
Moreover, using inverse Fourier transformation, similar as the method in section 5.3 of computing the value of $\beta$, we can use (inverse) Fourier transform to find $\theta_{k}$. Furthermore, it is easy to check $w_{0}$ and $w_{1}$ satisfy the equations in the theorem.

Remark 6.2.6. Notice that we have only proved the case there is one eigenvalue violating the attractor/repellent condition 6.2. If there are more eigenvalues violating (6.2), the conclusion is still true but the proof are much more complicate. In such situation, when we use the induction method, we do not have a proved base case as in two dimensional case. However, we can always use similar method as in section 5.4 to prove the base case first.

\subsubsection{Mixed type non-attracting case}

Here we will prove the case $n=3$, and by using the induction method we can prove the mixed type non-attracting case with one eigenvalue violating the attractor/repellent condition 6.2. Consider the normal form:

$$
L=\frac{\partial^{2}}{\partial x_{1}^{2}}+b_{1} x_{1}^{2} \frac{\partial^{2}}{\partial x_{3}^{2}}+\lambda x_{2} \frac{\partial^{2}}{\partial x_{2} \partial x_{3}}+x_{3} \frac{\partial^{2}}{\partial x_{3}^{2}}+\theta \frac{\partial}{\partial x_{3}}
$$

there is no integers $\left(t_{1}, t_{2}, t_{3}, t\right)$ such that

$$
t_{1}+t_{2} \lambda+t_{3}=\frac{t}{2}
$$

for $t \neq 0$.

Theorem 6.2.7. Let $u \in \mathscr{Q}\left(\mathbb{D}^{3}\right)$ such that $\left\{\left(0 ; 0^{\prime}, \xi_{3}\right) \mid \xi_{3}>0\right\} \subset \mathrm{WF}_{a}(u)$, then

$$
\mathrm{WF}_{a}(u) \cap \Gamma_{1} \neq \emptyset \quad \text { or } \quad \mathrm{WF}_{a}(u) \cap \Gamma_{2} \neq \emptyset .
$$

Proof. The proof is similar to the proof of Theorem 6.2.3. First we construct $u_{1}=P u$ for an elliptic microdifferential operator $P$, such that

$$
\begin{gathered}
\mathrm{WF}_{a}\left(u_{1}\right) \subset\left\{\left(0 ; 0^{\prime}, \xi_{3}\right) \mid \xi_{3}>0\right\}, \\
\hat{u}_{1}, \frac{\partial^{2}}{\partial \xi_{1}^{2}} \hat{u}_{1} \in \mathscr{Q}\left(\mathbb{D}^{3}\right) \cap L^{2}\left(\mathbb{R}^{3}\right), \\
(L-N) u_{1} \in \mathcal{A}_{*}\left(\mathbb{R}^{n}\right),
\end{gathered}
$$

and

$$
\operatorname{supp} \hat{u}_{1} \subset\left\{\xi_{3}>1,\left\|\xi^{\prime}\right\| \leq \epsilon \xi_{3}\right\}
$$

Theorem 6.2.8. Let $u_{1} \in \mathscr{Q}\left(\mathbb{D}^{3}\right) \cap L^{2}\left(\mathbb{R}^{3}\right)$ be a slowly increasing hyperfunction, and let $v_{0}, v_{1}, w_{0}, w_{1}$ be defined similarly as in section 6.2.1, then The following statements are equivalent:

(a) $\left\{\left(0 ; 0^{\prime}, \xi_{3}\right) \mid \xi_{3}>0\right\} \subset \mathrm{WF}_{a}\left(u_{1}\right)$, 
(b) $\left\{\left(0 ; 0^{\prime}, \xi_{3}\right) \mid \xi_{3}>0\right\} \subset \mathrm{WF}_{a}\left(v_{0}\right)$,

(c) $\left\{\left(0^{\prime} ; 0^{\prime \prime}, \xi_{3}\right) \mid \xi_{3}>0\right\} \subset \mathrm{WF}_{a}\left(w_{0}\right)$ or $\left\{\left(0^{\prime} ; 0^{\prime \prime}, \xi_{n}\right) \mid \xi_{n}>0\right\} \subset \mathrm{WF}_{a}\left(w_{1}\right)$, where $0^{\prime}$ and $0^{\prime \prime}$ are the origin in $\mathbb{R}^{2}$ and in $\mathbb{R}$ respectively. Moreover,

$$
L_{1}\left(\frac{\partial}{\partial x_{3}} w_{0}\right) \in \mathcal{A}\left(\mathbb{R}^{n-1}\right),
$$

where

$$
L_{1}=\lambda x_{2} \frac{\partial}{\partial x_{2}}+x_{3} \frac{\partial}{\partial x_{3}}+2\left(\operatorname{Re} \theta-\frac{1}{4}-2\right)
$$

Proof. The proof is almost the same as the proof of Theorem 6.2.5.

Remark 6.2.9. Notice that we have not proved $w_{1}$ satisfies some differential equation. Actually if $\left(0^{\prime} ; 0^{\prime \prime}, 1\right) \notin \mathrm{WF}_{a}\left(w_{0}\right)$ and $u_{1}$ solves $L u_{1}=f_{1}$, then we have $\left(0^{\prime} ; 0^{\prime \prime}, 1\right) \notin$ $\mathrm{WF}_{a}\left(w_{1}\right)$.

More specially, consider the operator $L$ in (6.11), and by taking Fourier transformation in both sides of $L u_{1}=f_{1}$ for some $f_{1} \in \mathcal{A}_{*}$, we have

$$
\begin{aligned}
\widehat{L u_{1}}= & {\left[-\xi_{1}^{2}-2 \sqrt{-1} \xi_{3}-\sqrt{-1} \xi_{3}^{2} \frac{\partial}{\partial \xi_{3}}+b_{1} \xi_{3}^{2} \frac{\partial^{2}}{\partial \xi_{1}^{2}}\right.} \\
& \left.+\sqrt{-1}(\theta-\lambda) \xi_{3}-\sqrt{-1} \lambda \xi_{3} \xi_{2} \frac{\partial}{\partial x_{2}}\right] \hat{u}_{1}=\hat{f}_{1} .
\end{aligned}
$$

Multiplying it by $\overline{\hat{u}_{1}}(\xi)$ and integrating in $\xi_{1}$, we have

$$
\begin{aligned}
& {\left[\int\left|\xi_{1} \hat{u}_{1}(\xi)\right|^{2} d \xi_{1}+\xi_{3}^{2} \int\left|\frac{\partial}{\partial \xi_{1}} \hat{u}_{1}\right|^{2} d \xi_{1}\right]} \\
& \leq(|\theta-\lambda|+2)\left|\xi_{3}\right| \int\left|\hat{u}_{1}\right|^{2}(\xi) d \xi_{1}+|\lambda|\left|\xi_{2}\right|\left|\xi_{3}\right|\left(\int\left|\frac{\partial}{\partial \xi_{2}} \hat{u}_{1}\right|^{2} d \xi_{1}\right)^{\frac{1}{2}}\left(\int\left|\hat{u}_{1}\right|^{2}(\xi) d \xi_{1}\right)^{\frac{1}{2}} \\
& +\xi_{3}^{2}\left(\int\left|\frac{\partial \hat{u}_{1}}{\partial \xi_{3}}\right|^{2} d \xi_{1}\right)^{\frac{1}{2}}\left(\int\left|\hat{u}_{1}\right|^{2}(\xi) d \xi_{1}\right)^{\frac{1}{2}}+\left(\int\left|\hat{f}_{1}\right|^{2}(\xi) d \xi_{1}\right)^{\frac{1}{2}}\left(\int\left|\hat{u}_{1}\right|^{2}(\xi) d \xi_{1}\right)^{\frac{1}{2}} .
\end{aligned}
$$

Then if $\int\left|\frac{\partial^{m}}{\partial \xi_{j}^{m}} \hat{u}_{1}(\xi)\right|^{2} d \xi_{1}$ are exponentially decreasing for $\left|\left(\xi_{2}, \xi_{3}\right)\right| \rightarrow \infty$ for $j=2,3$ and $m=0,1$, we have $\int\left|\frac{\partial \hat{u}_{1}}{\partial \xi_{1}}\right|^{2} d \xi_{1}$ is exponentially decreasing as $\left|\left(\xi_{2}, \xi_{3}\right)\right| \rightarrow \infty$. Notice that $\widehat{x_{j} u_{1}}=\frac{1}{\sqrt{-1}} \partial_{\xi_{j}} \hat{u}_{1}$.

\subsection{Solutions with prescribed singularities}

Consider the operator

$$
L=\sum_{\lambda=1}^{n-1} \lambda_{i} x_{i} \frac{\partial}{\partial x_{i}}+x_{n} \frac{\partial}{\partial x_{n}}+\theta
$$


suppose $\lambda_{1}<0$ and $\lambda_{i}>0$ for $2<i \leq n-1$. Write

$$
L_{1}=\frac{L}{\lambda_{1}}=x_{1} \frac{\partial}{\partial x_{1}}-\sum_{i=1}^{n-1} \mu_{i} x_{i+1} \frac{\partial}{\partial x_{i+1}}+\theta_{1},
$$

where

$$
\begin{gathered}
\mu_{i}=-\frac{\lambda_{i+1}}{\lambda_{1}} \quad \text { for } \quad 1<i \leq n-2, \\
\mu_{n-1}=-\frac{1}{\lambda_{1}} \quad \text { and } \quad \theta_{1}=\frac{\theta}{\lambda_{1}}
\end{gathered}
$$

We have $\mu_{i}>0$ for $1 \leq n \leq n-1$.

Proposition 6.3.1. Let $L_{1}$ be as before and let $u \in \mathscr{B}\left(\mathbb{R}^{n}\right)$ such that $L_{1} u=0$ and $\mathrm{WF}_{a}(u) \subset\left\{(x, \xi) \mid x_{1}=0\right\}$, then there is a decomposition

$$
u=u_{0}+u_{+}+u_{-}, \quad u_{0}, u_{+}, u_{-} \in \mathscr{B}\left(R^{n}\right)
$$

such that

$$
\begin{gathered}
\operatorname{supp} u_{0} \subset\left\{x \mid x_{1}=0\right\} \\
\operatorname{supp} u_{+} \subset\left\{x \mid x_{1} \geq 0\right\} \\
\operatorname{supp} u_{-} \subset\left\{x \mid x_{1} \leq 0\right\} \\
L_{1} u_{0}=L_{1} u_{+}=L_{1} u_{-}=0 .
\end{gathered}
$$

and $\left(0 ; 0^{\prime}, 1\right) \notin \mathrm{WF}_{a}\left(u_{+}\right) \cup \mathrm{WF}_{a}\left(u_{-}\right)$.

Proof. By the assumption on S. S.(u), the restrictions

$$
v=\left.u\right|_{x_{1}=1} \quad \text { and } \quad v^{\prime}=\left.u\right|_{x_{1}=-1}
$$

exist, and define two real analytic functions. Consider the Cauchy problem of integrating $v$ and $v^{\prime}$ along the bicharacteristics of $L_{1}$, Cauchy-Kovalevsky theorem gives the uniqueness of solution, we have

$$
u\left(x_{1}, \cdots, x_{n}\right)=x_{1}^{-\theta_{1}} v\left(x_{1}^{\mu_{1}} x_{2}, \cdots, x_{1}^{\mu_{n-1}} x_{n}\right) \quad \text { for } \quad x_{1}>0
$$

and

$$
u\left(x_{1}, \cdots, x_{n}\right)=x_{1}^{-\theta_{1}} v^{\prime}\left(x_{1}^{\mu_{1}} x_{2}, \cdots, x_{1}^{\mu_{n-1}} x_{n}\right) \quad \text { for } \quad x_{1}<0 .
$$

we can define the hyperfunction $u_{+}$and $u_{-}$as

$$
u_{+}\left(x_{1}, \cdots, x_{n}\right)=x_{1+}^{-\theta_{1}} v_{+}\left(x_{1+}^{\mu_{1}} x_{2}, \cdots, x_{1+}^{\mu_{n-1}} x_{n}\right) \quad \text { for } \quad x_{1}>0
$$

and

$$
u_{-}\left(x_{1}, \cdots, x_{n}\right)=x_{1-}^{-\theta_{1}} v_{-}^{\prime}\left(x_{1-}^{\mu_{1}} x_{2}, \cdots, x_{1-}^{\mu_{n-1}} x_{n}\right) \quad \text { for } \quad x_{1}<0 .
$$

We have $\operatorname{supp} u_{+} \subset\left\{x_{1} \geq 0\right\}, \operatorname{supp} u_{-} \subset\left\{x_{1} \leq 0\right\}$ and set $u_{0}=u-u_{+}-u_{-}$, then we have $\operatorname{supp} u_{0} \subset\left\{x_{1}=0\right\}$ and $L u_{+}=L u_{-}=L u_{0}=0$. Similar as in section 5.4. the condition on singular spectrum holds due to our assumption on $\mathrm{WF}_{a}(u)$. 
Proposition 6.3.2. Let $f \in \mathscr{B}\left(\mathbb{R}^{n}\right)$ such that $\operatorname{supp} f \subset\left\{x \mid x_{1}=0\right\}$, then for each $x_{0} \in \operatorname{supp} f$, there is an open neighborhood $\Omega$ of $x_{0}$, such that

$$
f=\sum_{j=0}^{\infty} \frac{\partial^{j}}{\partial x_{1}^{j}} f_{j} \quad \text { on } \quad \Omega
$$

where $f_{j} \in \mathscr{B}(\Omega)$ are uniquely determined with $\left\langle f_{j}, \phi\right\rangle=\left\langle f_{j}, \phi\left(0, \bullet^{\prime}\right)\right\rangle, \phi \in \mathcal{A}(\Omega)$.

Remark 6.3.3. Notice that $\mathscr{B}_{*}(\Omega)$ can be regarded as the dual space of $\mathcal{A}(\Omega)$. Moreover, in the case of a distribution $f \in \mathscr{D}^{\prime}\left(\mathbb{R}^{n}\right)$ the sum is finite, and the test function space is $\mathscr{D}$ instead of $\mathcal{A}$. In fact, a hyperfunction supported in $\left\{x_{1}=0\right\}$ can be written as $f=\left(\sum_{j=0}^{\infty} c_{j} \delta^{(j)}\left(x_{1}\right)\right) \otimes f\left(x^{\prime}\right)$, where

$$
\limsup _{j \rightarrow \infty} \sqrt[j]{j !\left|c_{j}\right|}=0 \text { for } c_{j} \in \mathbb{C}
$$

Apply the proposition to $u_{0}$, we have

$$
L_{1} u_{0}=L_{1}\left(\sum_{j=0}^{\infty} \frac{\partial^{j}}{\partial x_{1}^{j}} f_{j}\right)=0
$$

then

$$
\sum_{j=0}^{\infty} \frac{\partial^{j}}{\partial x_{1}^{j}}\left|\left(-\sum_{i=1}^{n-1} \mu_{i} x_{i+1} \frac{\partial}{\partial x_{i+1}}+(\theta-j-1)\right) f_{j}\right|=0 .
$$

Fix an arbitrary function $\phi$, then $f_{j}$ is determined, we have

$$
\left(-\sum_{i=1}^{n-1} \mu_{i} x_{i+1} \frac{\partial}{\partial x_{i+1}}+(\theta-j-1)\right) f_{j}=0, \forall j .
$$

If $\left\{\left(0 ; 0^{\prime}, \xi_{n}\right) \mid \xi_{n}>0\right\} \subset \mathrm{WF}_{a}\left(u_{0}\right)$, then there is $j_{0} \geq 0$, such that $\left\{\left(0 ; 0^{\prime}, \xi_{n}\right) \mid \xi_{n}>\right.$ $0\} \subset \mathrm{WF}_{a}\left(f_{j_{0}}\right)$. Choose the larger $j_{0}$ satisfying the condition. Near $\left(0 ; 0^{\prime}, 1\right)$, since $x_{1}^{j_{0}} u_{0}=\sum_{j=j_{0}}^{\infty}(-1)^{j_{0}} j_{0} ! \frac{\partial^{j-j_{0}}}{\partial x_{1}^{j-j_{0}}} f_{j}$, we have

$$
\mathrm{WF}_{a}\left(f_{j_{0}}\right)=\mathrm{WF}_{a}\left(x_{1}^{j_{0}} u_{0}\right) \subset \mathrm{WF}_{a}\left(u_{0}\right)
$$

Identify $f_{j_{0}}$ as a hyperfunction on $\mathbb{R}^{n-1}$. Let $L_{1}$ be as in Proposition 6.3.1, suppose also

$$
\mu_{i}>\mu_{n-1} \quad \text { for some } i, 1 \leq i \leq n-2
$$

we have

Proposition 6.3.4. Let $u$ be as in Proposition 6.3.1 such that $\left\{\left(0 ; 0^{\prime}, \xi_{n}\right) \mid \xi_{n}>0\right\} \subset$ $\mathrm{WF}_{a}(u)$. Then

$$
\mathrm{WF}_{a}(u) \cap \Gamma_{2} \cap \iota_{*}\left(T^{*} \mathbb{R}^{n-1}\right) \neq \emptyset,
$$


where $\Gamma_{2}$ is unstable manifold and $\iota_{*}$ is the push forward of

$$
\begin{gathered}
\iota: \mathbb{R}^{n-1} \rightarrow \mathbb{R}^{n} \\
\quad x^{\prime} \rightarrow\left(0, x^{\prime}\right) .
\end{gathered}
$$

For instance, if $\left\{\left(x^{\prime} ; \xi^{\prime \prime}, \xi_{n}\right) \mid \xi_{n}>0\right\} \subset \mathrm{WF}_{a}\left(f_{j_{0}}\right)$ near $\left\{\left(0^{\prime} ; 0^{\prime \prime}, \xi_{n}\right) \mid \xi_{n}>0\right\}$, then

$$
\left\{\left(0^{\prime} ; 0^{\prime \prime}, \xi_{n}\right) \mid \xi_{n}>0\right\} \subset \mathrm{WF}_{a}\left(f_{j_{0}}\right) \quad \text { and } \quad\left\{\left(0,0^{\prime} ; 0, \xi^{\prime \prime}, \xi_{n}\right) \mid \xi_{n}>0\right\} \subset \mathrm{WF}_{a}(u) \text {. }
$$

Remark 6.3.5. In Propositions 6.3.2 and 6.3.4 we construct solutions $u$ with $\mathrm{WF}_{a}(u) \cap$ $\Gamma_{1} \neq \emptyset$, and give an estimate of analytic singularities on unstable manifold $\Gamma_{2}$.

In three dimensional hyperbolic case

$$
L=\lambda_{1} x_{1} \frac{\partial}{\partial x_{1}}+\lambda_{2} x_{2} \frac{\partial}{\partial x_{2}}+x_{3} \frac{\partial}{\partial x_{3}}+\theta
$$

to satisfy the condition in Proposition 6.3.4, we need $\lambda_{1}, \lambda_{2} \notin[0,1]$.

According to Table 4.1, we have

\begin{tabular}{l|c|c|c|c|c|c|r}
\hline type & $x_{1}$ & $x_{2}$ & $x_{3}$ & $\xi_{1}$ & $\xi_{2}$ & $\xi_{3}$ & note \\
\hline (i) & $\pm e^{\lambda_{1} t}$ & 0 & 0 & 0 & 0 & 1 & $t \rightarrow \pm \infty$ \\
\hline (ii) & 0 & 0 & 0 & $\pm e^{\left(1-\lambda_{1}\right) t}$ & 0 & 1 & $t \rightarrow \pm \infty$ \\
\hline (iii) & 0 & 0 & 0 & 0 & $\pm e^{\left(1-\lambda_{2}\right) t}$ & 1 & $t \rightarrow \pm \infty$ \\
\hline (iv) & 0 & $\pm e^{\lambda_{2} t}$ & 0 & 0 & 0 & 1 & $t \rightarrow \pm \infty$ \\
\hline (v) & $\pm e^{b_{1} t}$ & $\pm e^{b_{2} t}$ & 0 & 0 & 0 & 1 & TBD \\
\hline (vi) & $\pm e^{\lambda_{1} t}$ & 0 & 0 & 0 & $\pm e^{\left(1-\lambda_{2}\right) t}$ & 1 & TBD \\
\hline (vii) & 0 & $\pm e^{\lambda_{2} t}$ & 0 & $\pm e^{\left(1-\lambda_{1}\right) t}$ & 0 & 1 & TBD \\
\hline (viii) & 0 & 0 & 0 & $\pm e^{\left(1-\lambda_{1}\right) t}$ & $\pm e^{\left(1-\lambda_{2}\right) t}$ & 1 & TBD \\
\hline
\end{tabular}

Table 6.1: List of projected null bicharacteristics, 3D hyperbolic case

Moreover, assume $\lambda_{1}<0$ and $\lambda_{2}>1$, and assume $\mathrm{WF}_{a}(u) \subset\left\{x_{1}=0\right\}$, then the stable manifold is $\Gamma_{1}=\left\{\left(x_{1}, 0,0, \xi_{2}\right)\right\}$ and the unstable manifold is $\Gamma_{2}=$ $\left\{\left(0, x_{2}, \xi_{1}, 0\right)\right\}$. Under those assumptions, the projected null bicharacteristics of type (vii) will intersect $\mathrm{WF}_{a}(u)$ if $\left(0 ; 0^{\prime}, 1\right) \in \mathrm{WF}_{a}(u)$. It is easy to check Proposition 6.3.4 holds.

Remark 6.3.6. In three dimensional hyperbolic non-attracting/non-repelling case 6-1b:

$$
L=\lambda_{1} x_{1} \frac{\partial}{\partial x_{1}}+\lambda_{2} x_{2} \frac{\partial}{\partial x_{2}}+x_{3} \frac{\partial}{\partial x_{3}}+\theta,
$$

where $\lambda_{1} \notin[0,1]$ and $0<\lambda_{2}<1$.

Assume $\lambda_{1}<0$, from Table 6.1 we have: The stable manifold is $\left\{\left(x_{1}, 0,0,0\right)\right\}$ and the unstable manifold is $\left\{\left(0, x_{2}, \xi_{1}, \xi_{2}\right)\right\}$. 
CHAPTER 6 Analytic singularities in higher dimensions

\begin{tabular}{l|c|c|c|c|c|c|r}
\hline type & $x_{1}$ & $x_{2}$ & $x_{3}$ & $\xi_{1}$ & $\xi_{2}$ & $\xi_{3}$ & note \\
\hline (i) & $\pm e^{\lambda_{1} t}$ & 0 & 0 & 0 & 0 & 1 & $t \rightarrow+\infty$ \\
\hline (ii) & 0 & 0 & 0 & $\pm e^{\left(1-\lambda_{1}\right) t}$ & 0 & 1 & $t \rightarrow-\infty$ \\
\hline (vii) & 0 & $\pm e^{\lambda_{2} t}$ & 0 & $\pm e^{\left(1-\lambda_{1}\right) t}$ & 0 & 1 & $t \rightarrow-\infty$ \\
\hline (viii) & 0 & 0 & 0 & $\pm e^{\left(1-\lambda_{1}\right) t}$ & $\pm e^{\left(1-\lambda_{2}\right) t}$ & 1 & $t \rightarrow-\infty$ \\
\hline
\end{tabular}

Table 6.2: List of projected null bicharacteristics, 3D hyperbolic case (6-1b)

Apply theorem 5.3.1 and Proposition 6.3.1, we can construct a solution $u_{0}=$ $\delta\left(x_{1}\right) \otimes w\left(x_{2}, x_{3}\right)$ supported in $\left\{x_{1}=0\right\}$, where $w$ is a solution of equation

$$
\lambda_{2} x_{2} \frac{\partial w}{\partial x_{2}}+x_{3} \frac{\partial w}{\partial x_{3}}+(\theta-1) w \in \mathcal{A}\left(\mathbb{R}^{2}\right)
$$

then $u_{0}$ satisfies $L u_{0}=0$ and $\mathrm{WF}_{a}(u)=\left\{\left(0,0,0 ; \xi_{1}, 0, \xi_{3}\right) \mid \xi_{3}>0\right\}$. Then near the radial point $u_{0}$ has minimal analytic singularity with $(0,0,0 ; 0,0,1) \in \mathrm{WF}_{a}\left(u_{0}\right)$, which is of type (ii) in Table 6.2 .

Remark 6.3.7. In $n$ dimensional case, if there is only one eigenvalues $\lambda_{1}$ of $B$ violates the condition 6.2), assume $\lambda_{1}<0$ and $\lambda_{i}>0,1<i \leq n-1$, according to the discussion in section 6.3 , we have the normal form $(6.12)$

$$
L_{1}=\frac{L}{\lambda_{1}}=x_{1} \frac{\partial}{\partial x_{1}}-\sum_{i=1}^{n-1} \mu_{i} x_{i+1} \frac{\partial}{\partial x_{i+1}}+\theta_{1},
$$

where $\mu_{i}<\mu_{n-1}$ for $1 \leq i \leq n-2$. Similarly, we can construct $u_{0}=\delta\left(x_{1}\right) \otimes$ $w\left(x_{2}, \cdots, x_{n}\right)$, where $w$ is a solution of equation

$$
\left(-\sum_{i=1}^{n-1} \mu_{i} x_{i+1} \frac{\partial}{\partial x_{i+1}}+\theta_{1}-1\right) w \in \mathcal{A}\left(\mathbb{R}^{n-1}\right),
$$

then $u_{0}$ satisfies $L_{1} u_{0}=0$ and $\mathrm{WF}_{a}(u)=\left\{\left(0,0,0 ; \xi_{1}, 0^{\prime \prime}, \xi_{n}\right) \mid \xi_{n}>0\right\}$, near the radial point $u_{0}$ has minimal analytic singularity with $\left(0 ; 0^{\prime}, 1\right) \in \mathrm{WF}_{a}\left(u_{0}\right)$.

Remark 6.3.8. In three dimensional hyperbolic non-attractor case $6-1 \mathrm{~b}$, we are going to construct solutions $u$ such that $\mathrm{WF}_{a}(u) \cap \Gamma_{1} \neq \emptyset$, we consider the normal form (6.12) with the condition (6.13). The stable manifold is $\left\{\left(x_{1}, 0,0,0\right)\right\}$ and the unstable manifold is $\left\{\left(0, x_{2}, \xi_{1}, \xi_{2}\right)\right\}$. From Table 6.2, we know $\mathrm{WF}_{a}(u) \cap(\mathrm{i}) \neq \emptyset$. Decompose $u=u_{+}+u_{0}+u_{-}$, we have $\mathrm{WF}_{a}\left(u_{+}\right) \cap$ (i) $\neq \emptyset$, i.e., we can construct a hyperfunction solution

$$
u_{+}\left(x_{1}, \cdots, x_{3}\right)=x_{1+}^{\theta_{1}} v\left(x_{1+}^{\mu_{1}} x_{2}, x_{1+}^{\mu_{2}} x_{3}\right) \quad \text { for } \quad x_{1}>0
$$

such that

$$
\mathrm{WF}_{a}\left(u_{+}\right) \cap\left\{x_{1} \neq 0\right\}=\left\{\left(e^{\lambda_{1} t}, 0,0,0, \xi_{3}\right) \mid \xi_{3}>0\right\} .
$$

Besides, there are at least two projected null bicharacteristics intersection the set

$$
\mathrm{WF}_{a}\left(u_{+}\right) \cap\left\{x_{1}=0\right\} .
$$


In additional, we have

(a) If one of projected null bicharacteristics of type (ii) is contained in $\mathrm{WF}_{a}(u)$, then the other one should be contained in $\mathrm{WF}_{a}(u)$ either.

(b) If one of projected null bicharacteristics of type (vii) is contained in $\mathrm{WF}_{a}(u)$, i.e., $\left(0, e^{\lambda_{2} t}, 0, e^{\left(1-\lambda_{1}\right) t}, 0,1\right)$, then the other one $\left(0, e^{\lambda_{2} t}, 0,-e^{\left(1-\lambda_{1}\right) t}, 0,1\right)$ should be contained in $\mathrm{WF}_{a}(u)$ either.

(c) If one of projected null bicharacteristics of type (viii) is contained in $\mathrm{WF}_{a}(u)$, then the other three should be contained in $\mathrm{WF}_{a}(u)$ either.

Theorem 6.3.9. In three dimensional hyperbolic non-attracting/non-repelling case 6-1c):

$$
L=\lambda_{1} x_{1} \frac{\partial}{\partial x_{1}}+\lambda_{2} x_{2} \frac{\partial}{\partial x_{2}}+x_{3} \frac{\partial}{\partial x_{3}}+\theta
$$

where $\lambda_{1}, \lambda_{2} \notin[0,1]$. Without loss of generality, assume $\lambda_{1}<0, \lambda_{2}>1$. If $\left(0 ; 0^{\prime}, 1\right) \in \mathrm{WF}_{a}(u)$, then at least two of the following projected null bicharacteristics will be contained in $\mathrm{WF}_{a}(u)$.

Proof. First from Table 6.1 we have:

\begin{tabular}{l|c|c|c|c|c|c|r}
\hline type & $x_{1}$ & $x_{2}$ & $x_{3}$ & $\xi_{1}$ & $\xi_{2}$ & $\xi_{3}$ & note \\
\hline (vi) & $\pm e^{\lambda_{1} t}$ & 0 & 0 & 0 & $\pm e^{\left(1-\lambda_{2}\right) t}$ & 1 & $t \rightarrow+\infty$ \\
\hline (vii) & 0 & $\pm e^{\lambda_{2} t}$ & 0 & $\pm e^{\left(1-\lambda_{1}\right) t}$ & 0 & 1 & $t \rightarrow-\infty$ \\
\hline
\end{tabular}

Table 6.3: List of projected null bicharacteristics, 3D hyperbolic case 6-1c)

There are 8 possible projected null bicharacteristics which go asymptotically to $(0,0,0 ; 0,0,1)$, i.e., type (vi) and (vii), while the other types are impossible to show up. The stable manifold is $\left\{\left(x_{1}, 0,0, \xi_{2}\right)\right\}$ and the unstable manifold is $\left\{\left(0, x_{2}, \xi_{1}, 0\right)\right\}$.

Since neither $\lambda_{1}$ nor $\lambda_{2}$ satisfy the condition (6.2), the projected null characteristics show up in both subspaces $\left(x_{1}, \xi_{1}\right)$ and $\left(x_{2}, \xi_{2}\right)$. The analytic wavefront set satisfies the estimate in Proposition 6.3.4.

Besides we have the conclusion:

If one of projected null bicharacteristics of type (vi) (respectively, type (vii)) is contained in $\mathrm{WF}_{a}(u)$, i.e., $\left(e^{\lambda_{1} t}, 0,0,0, e^{\left(1-\lambda_{2}\right) t}, 1\right)$ (respectively, $\left.\left(0, e^{\lambda_{2} t}, 0, e^{\left(1-\lambda_{1}\right) t}, 0,1\right)\right)$, then one of the others $\left(e^{\lambda_{1} t}, 0,0,0,-e^{\left(1-\lambda_{2}\right) t}, 1\right)$ (respectively, $\left.\left(0, e^{\lambda_{2} t}, 0,-e^{\left(1-\lambda_{1}\right) t}, 0,1\right)\right)$ should be contained in $\mathrm{WF}_{a}(u)$ either.

Theorem 6.3.10. In three dimensional loxodromic non-attracting/non-repelling case 6-1e):

$$
L=\lambda_{1} x_{1} \frac{\partial}{\partial x_{1}}+\lambda_{2} x_{2} \frac{\partial}{\partial x_{2}}+x_{3} \frac{\partial}{\partial x_{3}}+\theta,
$$

where $\lambda_{1}, \lambda_{2}$ are two conjugated complex with non-zero real part. Without loss of generality, assume $\operatorname{Re} \lambda_{1}>1$. If $\left(0 ; 0^{\prime}, 1\right) \in \mathrm{WF}_{a}(u)$, then at least one of the following projected null bicharacteristics will be contained in $\mathrm{WF}_{a}(u)$. 
Proof. From Table 6.1 we have:

\begin{tabular}{l|c|c|c|c|c|c|r}
\hline type & $x_{1}$ & $x_{2}$ & $x_{3}$ & $\xi_{1}$ & $\xi_{2}$ & $\xi_{3}$ & note \\
\hline $\mathrm{v}$ & $\pm e^{b_{1} t}$ & $\pm e^{b_{2} t}$ & 0 & 0 & 0 & 1 & $t \rightarrow-\infty$ \\
\hline viii & 0 & 0 & 0 & $\pm e^{\left(1-b_{1}\right) t}$ & $\pm e^{\left(1-b_{2}\right) t}$ & 1 & $t \rightarrow+\infty$ \\
\hline
\end{tabular}

Table 6.4: List of projected null bicharacteristics, 3D loxodromic case 6-1e

There are 8 possible projected null bicharacteristics which go asymptotically to $(0,0,0 ; 0,0,1)$, i.e., type (v) and (viii), while the others are impossible to show up. The stable manifold is $\left\{\left(0,0, \xi_{1}, \xi_{2}\right)\right\}$ and the unstable manifold is $\left\{\left(x_{1}, x_{2}, 0,0\right)\right\}$.

Besides we have the conclusion:

If one of projected null bicharacteristics of type (viii) is contained in $\mathrm{WF}_{a}(u)$, then the other three should be contained in $\mathrm{WF}_{a}(u)$ either.

Remark 6.3.11. In the beginning of this chapter, we have shown there are four non-attractor cases (6-1b), 6-1c), 6-1e) and (6-1h). Aside from the above three cases, we need to consider the mixed type non-attractor case 6-1h). However, Theorem 6.2.8 showed us the analytic singularities of the case (6-1h) has a very strong correlation with the two dimensional hyperbolic saddle case, and one can discuss about the propagation of analytic singularities similarly. 
Appendices 



\section{Appendix A}

\section{Symplectic geometry and contact geometry}

In sections A.1 and A.2, all the notions are standard, which can be found in plenty of literatures, such as Arnold [1], Silva [69], Sternberg [73] and so on. The main references of section A.3 are [40], [63] and [66]. The content of section A.4 are mainly from [32].

\section{A.1 Symplectic geometry}

Let $V$ be an m-dimensional vector space over $\mathbb{R}$ or $\mathbb{C}$, and let $\sigma: V \times V \rightarrow \mathbb{R}$ be a bilinear map. The map is skew-symmetric if $\sigma(u, v)=-\sigma(u, v)$ for all $u, v \in V$.

Theorem A.1.1. Let $\sigma$ be a skew-symmetric bilinear map on $V$. Then there is a basis $u_{1}, \cdots, c_{k}, e_{1}, \cdots, e_{n}, f_{1}, \cdots, f_{n}$ of $V$ such that

$$
\begin{array}{ll}
\sigma\left(u_{i}, v\right)=0, & \text { for all } i \text { and all } v \in V, \\
\sigma\left(e_{i}, e_{j}\right)=0=\sigma\left(f_{i}, f_{j}\right), & \text { for all } i, j, \text { and } \\
\sigma\left(e_{i}, f_{j}\right)=\delta_{i j}, & \text { for all } i, j .
\end{array}
$$

Denote the space spanned by $u_{1}, \cdots, u_{k}$ by $U$, and choose a complementary space $W$ to $U$ in $V$,

$$
V=U \oplus W
$$

Let $V^{*}$ be the dual space of $V$. The map $\tilde{\sigma}: V \rightarrow V^{*}$ is the linear map defined by $\tilde{\sigma}(v(u))=\sigma(v, u)$.

Definition A.1.2. A skew-symmetric bilinear map $\sigma$ is symplectic if $\tilde{\sigma}$ is bijective, i.e., $U=0$. The map $\sigma$ is called a linear symplectic structure on $V$, and $(V, \sigma)$ is called a symplectic vector space.

By Theorem A.1.1, the dimension of a symplectic vector space is even, $(V, \sigma)$ has a 
symplectic basis $e_{1}, \cdots, e_{n}, f_{1}, \cdots, f_{n}$ satisfying

$$
\sigma\left(e_{i}, f_{j}\right)=\delta_{i j} \quad \text { and } \quad \sigma\left(e_{i}, e_{j}\right)=0=\sigma\left(f_{i}, f_{j}\right)
$$

If for each $p \in M$, the map $\omega_{p}: T_{p} M \times T_{p} M \rightarrow \mathbb{R}$ is skew-symmetric bilinear and $\omega_{p}$ varies smoothly in $p$, then call the $\omega$ a de Rham 2-form on $M$.

The 2-form $\omega$ is symplectic if $\omega$ is closed and $\omega_{p}$ is symplectic for all $p \in M$.

Definition A.1.3. A symplectic manifold is a pair $(M, \omega)$ where $M$ is a manifold and $\omega$ is a symplectic form.

For a symplectic vector space $(E, \sigma)$ of dimension $2 n$, a vector space $W \subset V$ is said to be isotropic (resp. Lagrangian, resp. involutive) if $F \subset F^{\perp}$ (resp. $F=F^{\perp}$, resp. $F^{\perp} \subset F$ ). That is, $W \subset V$ is isotropic (resp. Lagrangian, resp. involutive), then $\operatorname{dim} W \leq n$ (resp. $=n$, resp. $\geq n)$. Moreover $W$ is Lagrangian if and only if $\operatorname{dim} W=\mathrm{n}$ and $W$ is both isotropic and involutive.

\section{A.2 Contact geometry}

Now we start to introduce some notions in contact geometry. Let $X$ be a $2 n+1$ dimensional manifold and let $L$ be a line subbundle of the cotangent bundle $T^{*} X$, let $L^{*}$ be its dual bundle and $L^{\perp}$ its orthogonal complement.

One can define a multi-linear homomorphism of vector bundles

$$
L^{\perp} \times L^{\perp} \times L \rightarrow \mathbb{C} \times X
$$

by

$$
\left(v_{1}, v_{2}, d \omega\right) \rightarrow\left\langle d \omega, v_{1} \wedge v_{2}\right\rangle
$$

and this provides an alternating bilinear homomorphism

$$
L^{\perp} \times L^{\perp} \rightarrow L^{\otimes-1}
$$

Definition A.2.1. Say $(X, L)$ is a contact manifold if the above map A.1 is non-degenerate.

Remark A.2.2. The above definition is equivalent to require the dimension of $X$ is odd and for a nowhere vanishing section $\alpha$ of $L$, the product

$$
\omega \wedge(d \omega)^{n-1}
$$

never vanishes and does not dependent on the choice of $\omega$, which will be called a fundamental 1-form, and under this definition we often write $(X, \omega)$ rather than $(X, L)$.

There is a strict relationship between symplectic and contact geometry. To be specific, write $\hat{X}=L^{*} \backslash X$, then for $s$, a cross-section of $\hat{X}$, define a 1-form $\vartheta$ on 
$\hat{X}$ by setting $s^{*}(\vartheta)=\omega$. Then $(d \vartheta)^{n}$ never vanishes and $\hat{X}$ is called the symplectic manifold associated with $(X, L)$, with canonical 1-form $\vartheta$.

Example A.2.3. Let $Y$ be an n-dimensional manifold $Y$, and $X=\mathbb{P}^{*} Y$ the projective cotangent bundle of $Y$. Then $\hat{X}=T^{*} Y \backslash Y$. The Darboux theorem states for a local coordinate system $\left(x_{1}, \cdots, x_{n}, p_{1}, \cdots, p_{n-1}\right)$ of $X$, there is a canonical 1 -form of the form

$$
\omega=d x_{n}-\left(p_{1} d x_{1}+\cdots+p_{n-1} d x_{n-1}\right) .
$$

And the associated symplectic manifold $\hat{X}$ has a local coordinate system $\left(x_{1}, \cdots, x_{n}, \eta_{1}, \cdots, \eta_{n}\right)$ with $p_{j}=-\frac{\eta_{j}}{\eta_{n}}$ for $j=1, \cdots, n-1$ and the symplectic structure is given by

$$
\vartheta=\eta_{1} d x_{1}+\cdots+\eta_{n} d x_{n}=\eta_{n} \omega
$$

This example shows us that every contact manifold is locally isomorphic to a projective cotangent bundle.

Definition A.2.4. Let $f, g$ be functions on a $2 n$ dimensional symplectic manifold $\hat{X}$. Their Poisson bracket is defined by

$$
\{f, g\}(d \vartheta) \triangleq n d f \wedge d g \wedge(d \vartheta)^{n-1} .
$$

Take a local coordinate system $(x, \eta)$ of $\hat{X}$, then

$$
\{f, g\}=\sum_{j=1}^{n}\left(\frac{\partial f}{\partial \eta_{j}} \frac{\partial g}{\partial x_{j}}-\frac{\partial f}{\partial x_{j}} \frac{\partial g}{\partial \eta_{j}}\right) .
$$

Definition A.2.5. Connecting with Poisson bracket, the Hamiltonian vector field is defined as:

$$
H_{f} \triangleq \sum_{j=1}^{n}\left(\frac{\partial f}{\partial \eta_{j}} \frac{\partial}{\partial x_{j}}-\frac{\partial f}{\partial x_{j}} \frac{\partial}{\partial \eta_{j}}\right) .
$$

By definition one has

$$
H_{\{f, g\}}=\left[H_{f}, H_{g}\right] .
$$

Definition A.2.6. An analytic subset $V$ of $T^{*} X$ is called involutive if $\left.f\right|_{V}=\left.g\right|_{V}=0$ implies $\left.\{f, g\}\right|_{V}=0$.

Theorem A.2.7. ([63], Chapter 2, theorem 5.3.2) For any coherent $\mathcal{E}_{X}$-module $\mathscr{M}$, $\operatorname{supp} \mathscr{M}$ is involutive.

That is to say, the support of a coherent $\mathcal{E}_{X}$-module has codimension less than or equal to $\operatorname{dim} X$.

Definition A.2.8. An involutive submanifold $V$ of $(X, \omega)$ is regular if $\omega$ never vanishes on $V$.

An analytic subset $A$ of $T^{*} X$ is called Lagrangian if $A$ is involutive and $\operatorname{dim} A=$ $\operatorname{dim} X$. A coherent $\mathcal{E}_{X}$-module is called holonomic (or maximally overdetermined) if its support is Lagrangian. 


\section{A.3 Quantized contact transformation}

Definition A.3.1. Let $\left(M, \omega_{M}\right)$ and $\left(N, \omega_{N}\right)$ be two contact manifolds of the same dimension, then a map $f$ from $X$ to $Y$ is a contact transformation if $f^{*} \omega_{N}$ is a fundamental 1-form for $X$.

Contact transformation refers to homogeneous symplectic homomorphism.

Definition A.3.2. Let $X$ and $Y$ be two (real or complex) manifolds with the same dimension, let $U$ and $V$ be two open subsets in $T^{*} X$ and $T^{*} Y$, and denote by $\alpha_{X}$ and $\alpha_{Y}$ the canonical 1-form on $T^{*} X$ and $T^{*} Y$, respectively. A diffeomorphism (a bi-holomorphic map in the complex case) $\varphi: U \rightarrow V$ is called a homogeneous symplectic transformation if $\varphi$ is homogeneous and $\varphi^{*} \alpha_{Y}=\alpha_{X}$.

If $\varphi$ is a homogeneous symplectic transformation, then $\varphi$ is a local isomorphism and is compatible with the action of $\mathbb{C}^{*}$. However, people works on algebraic analysis prefer to use ${ }^{1}$ the term "contact transformation" instead of homogeneous symplectic transformation.

Assume $Y=\mathbb{C}^{n}$ and let $\left(y_{1}, \cdots, y_{n} ; \eta_{1}, \cdots, \eta_{n}\right)$ be the coordinates of $T^{*} Y$, so that $\alpha_{Y}=\sum_{j} \eta_{j} d y_{j}$. Set $p_{j}=\eta_{j} \circ \varphi$ and $q_{j}=y_{j} \circ \varphi$, we have

(i) $\left\{p_{j}, p_{k}\right\}=\left\{q_{j}, q_{k}\right\}=0,\left\{p_{j}, q_{k}\right\}=\delta_{j k}$ for $j, k=1, \cdots, n$.

(ii) $p_{j}$ is homogeneous of degree 1 and $q_{j}$ is homogeneous of degree 0 with respect to the fiber coordinates.

In turn one can assume the function $\left\{q_{1}, \cdots, q_{n} ; p_{1}, \cdots, p_{n}\right\}$ on $U \subset T^{*} X$ satisfy the above conditions (i) and (ii), then the map

$$
\begin{aligned}
\varphi: U & \rightarrow T^{*} Y \\
x & \mapsto\left\{q_{1}(x), \cdots, q_{n}(x) ; p_{1}(x), \cdots, p_{n}(x)\right\} \in T^{*} Y .
\end{aligned}
$$

is a homogeneous symplectic transformation. And we call $\left\{q_{1}, \cdots, q_{n} ; p_{1}, \cdots, p_{n}\right\}$ a homogeneous symplectic coordinate system.

We give a rather abstract definition of contact transformation, there is another intuitive way to construct contact transformations, which has been mentioned by Egorov [16], Hörmander [29] and Maslov [58], also can be found in Kashiwara [38], [KKK] [40], Kato-Struppa [45], [SKK] [63] and so on.

Let $M$ and $N$ be two open subset of $\mathbb{C}^{n}$, and let $\Lambda$ be the non-singular hypersurface of $M \times N$ defined by some holomorphic function $\Gamma(x, y)=0$, here non-singular means $\nabla_{x, y} \Gamma(x, y) \neq 0$ on $\Lambda$. Assume the determinate of the $(n+1) \times(n+1)$ matrix

$$
\left(\begin{array}{cc}
0 & d_{y} \Gamma \\
d_{x} \Gamma & d_{x} d_{y} \Gamma
\end{array}\right)
$$

\footnotetext{
${ }^{1}$ See Schapira [66], Page 176.
} 
does not vanish on $\Lambda$. Then one can construct a contact transformation from $P^{*} M$ to $P^{*} N$ via

$$
\begin{aligned}
P_{\Lambda}^{*}(M \times N)=\left\{(x, y ; \xi, \eta) \in P^{*}(M \times N) \mid \Gamma(x, y)\right. & =0 \\
\text { and }(\xi, \eta) & \left.=c \nabla_{x, y} \Gamma(x, y) \quad \text { for } \quad c \neq 0\right\}
\end{aligned}
$$

The implicit function theorem implies that

$$
\pi_{1}: P_{\Lambda}^{*}(M \times N) \rightarrow P^{*} M
$$

and

$$
\pi_{2}: P_{\Lambda}^{*}(M \times N) \rightarrow P^{*} N
$$

are local isomorphisms.

Definition A.3.3. The local isomorphisms

$$
\pi_{1} \circ \pi_{2}^{-1}: P^{*} N \rightarrow P^{*} M
$$

and

$$
\pi_{2} \circ \pi_{1}^{-1}: P^{*} M \rightarrow P^{*} N
$$

are called contact transformations having $\Lambda$ as a generating function.

Classical results showed that every contact transformation can be expressed by composition of two contact transformations with generating functions.

Theorem A.3.4. ([63] Chap. II §3.2) Let $\varphi: T^{*} X \supset U \rightarrow T^{*} Y$ be a homogeneous symplectic transformation, let $p_{X}$ be a point of $U$ and set $p_{Y}=\varphi\left(p_{X}\right)$. Then we have

(a) There exists an open neighborhood $U^{\prime}$ of $p_{X}$ and a $\mathbb{C}$-algebra isomorphism $\Phi$ : $\left.\left.\varphi^{-1} \mathcal{E}_{Y}\right|_{U^{\prime}} \rightarrow \mathcal{E}_{X}\right|_{U}$ (we call $(\varphi, \Phi)$ a quantized contact transformation).

(b) If $\Phi:\left.\varphi_{Y}^{-1} \rightarrow \mathcal{E}_{X}\right|_{U}$ is a $\mathbb{C}$-algebra isomorphism, then for any $m$, $\Phi$ gives an isomorphism $\left.\varphi^{-1} \mathcal{E}_{Y}(m) \rightarrow \mathcal{E}_{X}(m)\right|_{U}$. Moreover the following diagram commutes:

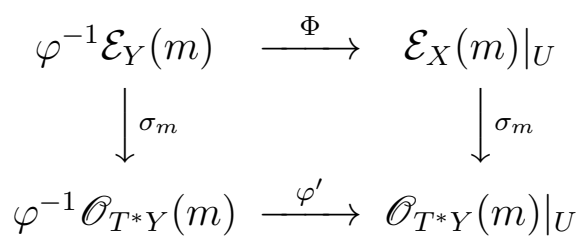

(c) Let $\Phi$ and $\Phi^{\prime}$ be two $\mathbb{C}$-algebra homomorphisms $\left.\varphi^{-1} \mathcal{E}_{Y} \rightarrow \mathcal{E}_{X}\right|_{U}$. Then there exist $\mu \in \mathbb{C}$, a neighborhood $U^{\prime}$ of $p_{X}$ and $P \in \Gamma\left(U ; \mathcal{E}_{X}(\mu)\right)$ such that $\sigma_{\mu}(P)$ is invertible and

$$
\Phi^{\prime}(Q)=P \Phi(Q) P^{-1} \quad \text { and }\left.\quad Q \in \varphi^{-1} \mathcal{E}_{Y}\right|_{U^{\prime}}
$$

Moreover $\mu$ is unique and $P$ is unique up to a constant multiple. 
(d) Let $P_{j} \in \Gamma\left(U ; \mathcal{E}_{X}(1)\right)$ and $Q_{j} \in \Gamma\left(U ; \mathcal{E}_{X}(0)\right)(1 \leq j \leq n)$ satisfy

$$
\left[P_{j}, P_{k}\right]=\left[Q_{j}, Q_{k}\right]=0, \quad\left[P_{j}, Q_{k}\right]=\delta_{j} k,
$$

then there exists a unique quantized contact transformation $(\varphi, \Phi)$ such that

$$
\varphi(p)=\left(\sigma_{0}\left(Q_{1}\right)(p), \cdots, \sigma_{0}\left(Q_{n}\right)(p), \sigma_{1}\left(P_{1}\right)(p), \cdots, \sigma_{1}\left(P_{n}\right)(p)\right),
$$

and $\Phi\left(y_{j}\right)=Q_{j}, \Phi\left(\partial_{y_{j}}\right)=P_{j}$. We call $\left\{Q_{1}, \cdots, Q_{n}, P_{1}, \cdots, P_{n}\right\}$ quantized canonical coordinates.

We give some examples of quantized contact transformations. More examples can be found in [38], [40] and [45].

Example A.3.5. ([38], Example 7.3.1) For instance, the quantized canonical coordinates of a constant coefficient micro-differential operator $P(x, \partial)$ of first order is given by

$$
\varphi(p)=\left(x_{1}+\left[P, x_{1}\right], x_{2}+\left[P, x_{2}\right], \cdots, x_{n}+\left[P, x_{n}\right], \partial_{x_{1}}, \cdots, \partial_{x_{n}}\right) .
$$

Example A.3.6. The most classical contact transformation with generating function is Legendre transformation. The generating function is

$$
\Omega(x, y)=x_{n}-y_{n}+\sum_{j=1}^{n-1} x_{j} y_{j}
$$

and we have a contact transformation $\varphi:(x, \xi) \rightarrow(y, \eta)$ with

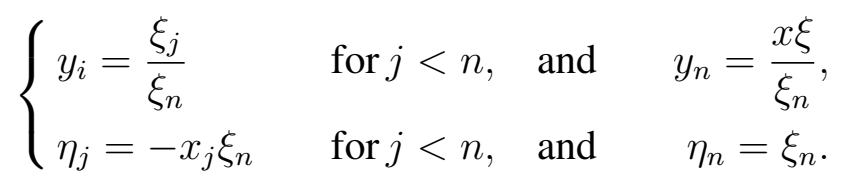

The related quantized contact transformation is given by

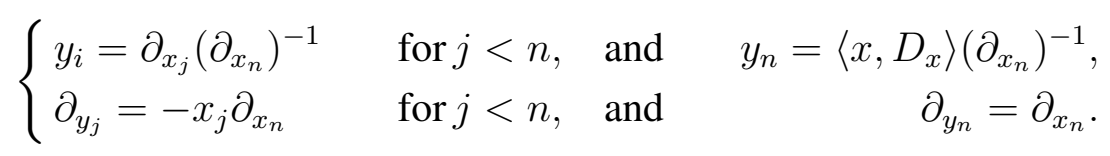

Quantized contact transformations are the lifting of contact transformations from the manifolds on which they act to the sheaves of differential (and microdifferential) operators on related manifolds. Moreover, given a contact transformation $\varphi$ from an open set $U \in T^{*} X$ to an open set $U^{\prime} \in T^{*} X^{\prime}, \varphi$ can be locally quantized, that is lifted to an isomorphism $\hat{\varphi}$ of filtered rings from $\left.\mathcal{E}_{X}\right|_{U}$ to $\left.\mathcal{E}_{X^{\prime}}\right|_{U^{\prime}}$.

Theorem A.3.7. ([40] Page 221.) Let $M$ and $N$ be real analytic manifolds of dimension $n$. Assume that a real-valued real analytic function $\Gamma(x, y)$ defined on $M \times N$ satisfies the above conditions. Then, for an arbitrary micro-differential operator $P\left(x, D_{x}\right), a$ 
micro-differential operator $Q\left(y, D_{y}\right)$ is uniquely determined such that

$$
\int P\left(x, D_{x}\right) \delta(\Gamma(x, y)) u(y) d y \simeq \int \delta(\Gamma(x, y)) Q\left(y, D_{y}\right) u(y) d y
$$

holds for any microfunction $u(y)$. Conversely, if $Q$ is given, then $P$ is uniquely determined so that the above formula holds. Moreover, the order of $Q$ and $P$ are equal. That is, we have sheaf isomorphisms:

$$
\begin{aligned}
p^{-1} \mathcal{E}_{M} & \cong q^{-1} \mathcal{E}_{N}, \\
p^{-1} \mathcal{E}_{M}(m) & \cong q^{-1} \mathcal{E}_{N}(m)
\end{aligned}
$$

and

$$
p^{-1} \mathcal{A}_{S^{*} M} \cong q^{-1} \mathcal{A}_{S^{*} N},
$$

where $p$ is the map producing $P$ by giving $Q$ and $q$ is the map producing $Q$ by giving $P$.

Remark A.3.8. ([40] Page 226.) One notice that there are plenty of choices of kernel functions instead of $\delta(\Gamma(x, y))$. The isomorphism of above formula is unique up to an inner automorphism by an invertible micro-differential operator of order zero. That is, one can use any non-degenerate section of a simple holonomic system with its characteristic variety being the conormal bundle of $H$, instead of $\delta(\Gamma(x, y))$.

\section{A.4 Linearization of real analytic vector field}

Suppose a real analytic vector field $V$ in $\mathbb{R}^{n}$ under the local coordinates $x=$ $\left(x_{1}, \cdots, x_{n}\right)$ be of the following form:

$$
V=\sum_{i=1}^{n} f_{i}(x) \frac{\partial}{\partial x_{i}},
$$

where all $f_{i}(x)^{\prime} s$ are real analytic functions defined in some neighborhood of the origin and $f_{i}(x)=0, \forall i=1, \cdots, n$. Actually, one can write $V$ in the form of

$$
V=\sum_{i, j=1}^{n} a_{i j} x_{i} \frac{\partial}{\partial x_{j}}+\text { higher order terms },
$$

and let $\lambda_{1}, \cdots, \lambda_{n}$ be the eigenvalues of the matrix $\left(a_{i j}\right)$. It is well known that all the eigenvalues do not depend on the choice of coordinates.

That is, one can choice suitable local coordinates to make

$$
f_{i}(x)=\lambda_{i} x_{i}+\text { higher order terms. }
$$

For a single analytic vector field $V$ in $\mathbb{C}^{n}$ vanishing at zero, Henri Poincaré [62] showed us: 
Theorem A.4.1. If one has the following conditions:

(i) The Jacobian matrix $\left(\left.\frac{\partial f_{i}}{\partial x_{j}}\right|_{x=0}\right)_{i, j}$ is diagnoseable with eigenvalues $\left\{\lambda_{j}\right\}_{j=1}^{n}$,

(ii) there are no nonnegative integers solutions of the equation

$$
\lambda_{i}=\sum_{j=1}^{n} k_{j} \lambda_{j}
$$

for $\sum_{j=1}^{n} k_{j}>1$,

(iii) the convex hull of the family of all eigenvalues $\left\{\lambda_{1}, \cdots, \lambda_{n}\right\}$ does not contain the origin, i.e., all the $\lambda_{i}^{\prime}$ s lie in the same open half-plane about the origin,

then one can find an analytic change of coordinates

$$
\varphi:\left(\mathbb{R}^{n}, 0\right) \rightarrow\left(\mathbb{R}^{n}, 0\right), x \mapsto y
$$

such that

$$
\varphi_{*} V=\sum_{i=1}^{n} \lambda_{i} y_{i} \frac{\partial}{\partial y_{j}}
$$

Remark A.4.2. Condition (ii) is called the non-resonance condition, which guarantees a formal Taylor's series development for the linearizing map, and condition (iii) guarantees the convergence of the formal series. Moreover, condition (ii) is required in most of the related linearization theorems, which can be seen from an analysis in Sternberg's paper [72]. Chen removed condition (i) entirely in [10], and both Sternberg [72] and Chen [10] provided the smooth versions of Poincaré's theorem without the restriction of (iii). However, it seems that we can only unwind but can not remove the condition (iii) in (real) analytic case. That is one main difference between smooth setting and real analytic setting for our problem.

Remark A.4.3. Indeed Henri Poincaré's original work is about analytic vector field, for a real analytic vector field vanishing at zero, the proof is similar. We have a sketch of a proof of Poincaré's result following from Y.Ilyashenko and S. Yakovenko [32] here to give a clear idea to proof the linearization theory in real analytic settings, for detail, see Poincaré[62], Shalomo Sternberg[71].

Definition A.4.4. An ordered tuple of complex numbers $\lambda=\left(\lambda_{1}, \cdots, \lambda_{n}\right) \in \mathbb{C}^{n}$ is called resonant, if there exist non-negative integers $\alpha=\left(\alpha_{1}, \cdots, \alpha_{n}\right) \in \mathbb{Z}_{+}^{n}$ such that $|\alpha|>1$ and the resonance identity occurs,

$$
\lambda_{j}=\langle k, \lambda\rangle, \quad|\alpha|>1
$$

Here $\langle\alpha, \lambda\rangle=\alpha_{1} \lambda_{1}+\cdots+\alpha_{n} \lambda_{n}$. The natural number $|\alpha|$ is the order of the resonance.

A square matrix is resonant if the collection of its eigenvalues (with repetitions if they are multiple) is resonant, otherwise it is non-resonant. 
Definition A.4.5. The Poincaré domain $\mathfrak{P} \subset \mathbb{C}^{n}$ is the collection of all tuples $\lambda=$ $\left(\lambda_{1}, \cdots, \lambda_{n}\right)$ such that the convex hull of the point set $\left\{\lambda_{1}, \cdots, \lambda_{n}\right\} \subset \mathbb{C}$ does not contain the origin inside or on the boundary. The Siegel domain $\mathfrak{S}$ is the complement to the Poincaré domain in $\mathbb{C}^{n}$.

Remark A.4.6. Sometimes we call such tuples as being of Poincaré type.

Theorem A.4.7. (Poincaré) A non-resonant holomorphic vector field with the linear part of Poincaré type can be linearized by a holomorphic transformation.

Remark A.4.8. Here, for the phase " linear part of Poincaré type", we mean eigenvalues of the related Jacobean matrix of the linear part are of Poincaré (resp. Siegel) type.

Suppose an analytic vector field $V$ in $\mathbb{C}^{n}$ under the local coordinates $x=$ $\left(x_{1}, \cdots, x_{n}\right)$ be of the following form:

$$
V=\sum_{i=1}^{n} f_{i}(x) \frac{\partial}{\partial x_{i}},
$$

where all $f_{i}(x)^{\prime} s$ are analytic functions defined in some neighborhood of the origin and $f_{i}(x)=0, \forall i=1, \cdots, n$. Then

$$
f_{i}(x)=\sum_{n=0}^{\infty} \frac{f^{(n)}(0)}{n !} x^{n}=f^{\prime}(0) x+O\left(|x|^{2}\right)
$$

Definition A.4.9. Two formal vector fields $F, F^{\prime}$ are formally equivalent, if there exists an invertible formal automorphism $H$ such that the

$$
H_{*} \cdot F(x)=F(H(x)), \quad H_{*}=\left(\frac{\partial H}{\partial x}\right)
$$

Theorem A.4.10. A non-resonant formal vector field $F(x)=A x+\cdots$ is formally equivalent to its linearization $F^{\prime}(x)=A x$.

Proof. Let $F(x)=A x+V_{m}(x)+V_{m+1}(x)+\cdots$, where $V_{i}, i=m, m+1, \cdots$ are arbitrary homogeneous vector fields of degrees $i$, here $m \geq 2$.

First want to remove $V_{m}$, and $F$ is formally equivalent to the formal field $F^{\prime}(x)=$ $A x+V_{m+1}^{\prime}(x)+\cdots$.

Choose $H(x)=x+P_{m}(x)$, where $P_{m}$ is homogeneous vector polynomial of degree $m$. The Jacobian matrix of $H(x)$ is $I+\left(\frac{\partial P_{m}}{\partial x}\right)$.

Then the conjugacy $H$, one has $H \circ F^{\prime}=F \circ H$ :

$$
\left(I+\frac{\partial P_{m}}{\partial x}\right)\left(A x+V_{m}(x)+\cdots\right)=A\left(x+P_{m}(x)\right)+V_{m}^{\prime}\left(x+P_{m}(x)\right)+\cdots .
$$

The homogeneous term of order 1 on both side coincide. To meet the condition $V_{m}^{\prime}=0, P_{m}$ must satisfy

$$
\left[\mathbf{A}, P_{m}\right]=-V_{m}, \mathbf{A}(x)=A x
$$


where $\mathbf{A}=A x$ is the linear vector field, the principal part of $F$, and the commutator

$$
\left[\mathbf{A}, P_{m}\right]=\left(\frac{\partial P_{m}}{\partial x}\right) \cdot A x-A P(x)
$$

Definition A.4.11. Let $\mathbf{A}(x)=A x$ be a linear vector field and let $P$ be a homogeneous vector polynomial. Denote the operator $\mathrm{ad}_{A}$ by

$$
\operatorname{ad}_{A}: P \rightarrow[\mathbf{A}, P], \quad\left(\operatorname{ad}_{A} P\right)(x)=\left(\frac{\partial P}{\partial x}\right) \cdot A x-A P(x) .
$$

Lemma A.4.12. ([32], Lemma 4.5) If $A$ is non-resonant, then the operator $\operatorname{ad}_{A}$ is invertible.

Proof. The assertion of the lemma is completely transparent when $A$ is a diagonal matrix $\Lambda=\operatorname{diag}\left\{\lambda_{1}, \cdots, \lambda_{n}\right\}$, then one knows $a d_{\Lambda}$ has $n$ eigenvalues $\langle\lambda, \alpha\rangle-\lambda_{k}, k=$ $1, \cdots, n$, with corresponding eigenvectors $F_{k \alpha}=x^{\alpha}(0, \cdots, 1, \cdots, 0)^{T}$. In fact, we have $\Lambda F_{k \alpha}=\lambda_{k} F_{k \alpha}$ and $\left(\frac{\partial F_{k \alpha}}{\partial x}\right) \Lambda x=\langle\lambda, \alpha\rangle F_{k \alpha}$.

Use the above lemma,

$$
\left[\mathbf{A}, P_{m}\right]=-V_{m}, \mathbf{A}(x)=A x
$$

is always solvable for arbitrary $V_{m}$.

Repeating this process inductively, one can construct an infinite sequence of polynomial maps $H_{1}, H_{2}, \cdots, H_{m}, \cdots$ and the formal fields $F_{1}=F, F_{2}, \cdots, F_{m}, \cdots$ such that

$$
F_{m}=A x+(\text { terms of order larger or equal } m)
$$

and the transformation

$$
H_{m}=i d+(\text { terms of order larger or equal } m)
$$

conjugates the $F_{m}$ with $F_{m+1}$.

Thus the composition $H^{m}=H_{m} \circ \cdots \circ H_{1}$ conjugates $F_{1}$ and $F_{m+1}$ without nonlinear terms up to order $m$.

The limit

$$
H=H^{\infty}=\lim _{m \rightarrow \infty} H^{(m)}
$$

exists in the class of formal morphisms. By construction, $H_{*} F$ cannot contain any nonlinear terms and hence is linear as required.

We have shown the formal linearization for holomorphic vector field, similarly we have the formal linearization for real analytic vector field:

Theorem A.4.13. ([54], Theorem 4) Let $V$ be a real analytic vector field on $\mathbb{R}^{n}$ satisfies the above conditions (i) and (ii) in Theorem A.4.1 then there exists a formal power series for a linearizing map for $V$ about the origin. 
Remark A.4.14. Since we already construct formal linearization map for a non-resonant real analytic vector field, the left question is whether the formal morphism is convergent to a real analytic map with suitable condition. We would like to discuss it in two cases, eigenvalues of Poicaré type and eigenvalues of Siegel type.

Proposition A.4.15. ([32], Proposition 5.2) If $\lambda=\left(\lambda_{1} \cdots, \lambda_{n}\right)$ is of Poicaré type, then only finitely many denominators ${ }^{2} \lambda_{j}-\langle\alpha, \lambda\rangle, \alpha \in \mathbb{Z}_{+}^{n},|\alpha| \geq 2$, may actually vanish. Moreover, nonzero denominators are bounded away from the origin: the origin is an isolated point of the set of all denominators $\left\{\lambda_{j}-\langle\alpha, \lambda\rangle, j=1, \cdots, n,|\alpha| \geq 2\right\}$.

If $\lambda$ is of Siegel type, then either there are infinitely many vanishing denominators, or the origin 0 is their accumulation point.

Proof. If the convex hull of $\left\{\lambda_{1}, \cdots, \lambda_{n}\right\}$ does not contain the origin, by the convex separation theorem, there exists a real linear functional $\ell: \mathbb{C} \rightarrow \mathbb{R}$ such that $\ell\left(\lambda_{j}\right) \leq$ $-r<0$ for all $\lambda_{j}$, and hence $\ell(\langle\alpha, \lambda\rangle) \leq-r|\alpha|$. Then

$$
\ell\left(\lambda_{j}-\langle\alpha, \lambda\rangle\right) \geq \ell\left(\lambda_{j}\right)+|\alpha| r \rightarrow \infty \quad|\alpha| \rightarrow \infty .
$$

Since $\ell$ is bounded on any small neighborhood of the origin, then the assertions are proved.

For $\lambda$ of Siegel type, please check [32], Proposition 5.2 for detail.

Now we try to finish the proof of Poincaré's Theorem A.4.7 for vector field with a diagonal non-resonant linear part $\Lambda=\operatorname{diag}\left\{\lambda_{1}, \cdots, \lambda_{n}\right\}$.

The classical proof by Poincaré was achieved by the so called Majorant method, and in modern language, it takes a more convenient form of the contracting map principle in an appropriate functional space, the majorant space.

Definition A.4.16. The majorant operator is the nonlinear operator acting on formal series by replacing all Taylor coefficients by their absolute values,

$$
\mathbf{M}: \sum_{k \in \mathbb{Z}_{n}^{+}} c_{k} z^{k} \mapsto \sum_{k \in \mathbb{Z}_{n}^{+}}\left|c_{k}\right| z^{k} .
$$

The action of the majorant operator naturally extends on all formal objects, such as vector formal series, formal vector fields, formal transformations, etc.

Definition A.4.17. The majorant $\rho$-norm is the functional on the space of formal power series $\mathbb{C}\left[\left[z_{1}, \cdots, z_{n}\right]\right]$, defined as

$$
\|f\|_{\rho}=\sup _{|z|<\rho}|\mathbf{M}(f(z))|=|\mathbf{M} f(\rho, \cdots, \rho)| \leq+\infty .
$$

For a formal vector function $F=\left(F_{1}, \cdots, F_{n}\right)$, then

$$
\|F\|_{\rho}=\left\|F_{1}\right\|_{\rho}+\cdots+\left\|F_{n}\right\|_{\rho} .
$$

\footnotetext{
${ }^{2}$ We call it denominator since it becomes the denominator part when we consider the inverse of the operator $a d_{\Lambda}$, see Lemma A.4.19
} 
The majorant space $\mathfrak{M}_{\rho}$ is the space of formal vector functions from $\mathbb{C}[[x]]$ having finite majorant $\rho$-norm.

Proposition A.4.18. The space $\mathfrak{M}_{\rho}$ with the majorant norm $\|\cdot\|_{\rho}$ is complete.

Lemma A.4.19. If $\Lambda \in M(n, \mathbb{C})$ is a non-resonant diagonal matrix of Poincaré type, then the operator ad $d_{\Lambda}$ has a bounded inverse in the space of vector fields equipped with the majorant norm.

Proof. The formal inverse operator $\operatorname{ad}_{\Lambda}^{-1}$ is diagonal,

$$
\operatorname{ad}_{\Lambda}^{-1}: \sum_{k, \alpha} c_{k \alpha} x^{\alpha} \frac{\partial}{\partial x_{k}} \mapsto \sum_{k, \alpha} \frac{c_{k \alpha} x^{\alpha}}{\lambda_{k}-\langle\alpha, \lambda\rangle} \frac{\partial}{\partial x_{k}}
$$

Let $F=\left(F_{1}, \cdots, F_{n}\right) \in D\left(\mathbb{C}^{n}, 0\right)$ be a holomorphic vector function defined in some polydisk near the origin. The operator of argument shift is the operator

$$
S_{F}: h(x) \mapsto F(x+h(x)),
$$

acting on holomorphic vector fields $h \in D\left(\mathbb{C}^{n}, 0\right)$ without the free term, $h(0)=0$.

Consider the one-parameter family of majorant Banach spaces $B_{\rho}$ indexed by the real parameter $\rho \in\left(\mathbb{R}_{+}^{n}, 0\right)$. We consider $B_{\rho^{\prime}}$ as a subspace in $B_{\rho}$ for all $0<\rho<\rho^{\prime}$.

Let $S$ be an operator defined on all of these spaces for all sufficiently small values of $\rho$, as a family of operators $S_{\rho}: B_{\rho} \rightarrow B_{\rho}$.

Definition A.4.20. The operator $S=\left\{S_{\rho}\right\}$ is strongly contracting, if

(1) $\|S(0)\|_{\rho}=O\left(\rho^{2}\right)$ and

(2) $S$ is Lipschitz on the ball $\tilde{B}_{\rho} \subset B_{\rho}$ of the majorant $\rho$-norm (with the same $r h o$ ), with the Lipschitz constant no greater than $O(\rho)$ as $\rho \rightarrow 0$.

Notice that any strongly contracting operator takes the balls $\tilde{B}_{\rho}$ strictly into themselves, since the center of the ball is shifted by $O\left(\rho^{2}\right)$ and the diameter of the image $S\left(\tilde{B}_{\rho}\right)$ does not exceed $2 \rho O(\rho)=O\left(\rho^{2}\right)$.

In the Poicaré domain the absolute values of all denominators are bounded from below by a positive constant $\varepsilon>0$, therefore any majorant $\rho$-norm is increased by no more than $\varepsilon^{-1}$ :

$$
\left\|\operatorname{ad}_{\Lambda}^{-1}\right\|_{\rho} \leq\left(\inf _{j, \alpha}\left|\lambda_{j}-\langle\alpha, \lambda\rangle\right|\right)^{-1}<+\infty
$$

Proof. Now we try to prove a holomorphic vector field with diagonal non-resonant linearization matrix $\Lambda$ of Poincaré type is holomorphically linearizable in a sufficiently small neighborhood of the origin. 
A holomorphic transformation $H=i d+h$ conjugates the linear vector field $\Lambda x$ with the initial nonlinear field $\Lambda x+F(x)$, if and only if

$$
\frac{\partial h}{\partial x} \Lambda x-\Lambda h(x)=F(x+h(x)) .
$$

that is

$$
\operatorname{ad}_{\Lambda} h=S_{F} h=F \circ(i d+h), \quad \operatorname{ad}_{\Lambda}=[\Lambda, \cdot] .
$$

And then if one can show the operator $\operatorname{ad}_{\Lambda}^{-1} \circ S_{F}$ restricted on the space $\mathfrak{M}_{\rho}$ has a fixed point $h$ for sufficient small $\rho$, then one can finish the proof.

Consider this operator $\operatorname{ad}_{\Lambda}^{-1} \circ S_{F}$ in the space $\mathfrak{M}_{\rho}$, with sufficient small $\rho$. Firstly the operator $\operatorname{ad}_{\Lambda}^{-1}$ is bounded, its norm is the reciprocal to the smallest small divisor and is independent of $\rho$. On the other hand, the shift operator $S_{F}$ is strongly contracting with the contraction rate (Lipschitz constant) going to zero with $\rho$ as $O(\rho)$. Thus the composition will be contracting on the $\rho$-ball $B_{\rho}$ in the $\rho$-majorant norm with the contraction rate $O(1) \cdot O(\rho)=O(\rho) \rightarrow 0$. By the contracting map principle, there exists a unique fixed point of the operator equation

$$
h=\left(\operatorname{ad}_{\Lambda}^{-1} \circ S_{F}\right)(h)
$$

in the space $\mathfrak{M}_{\rho}$ which is therefore a holomorphic vector function. The corresponding map $H$ linearizes the holomorphic vector field.

Apply the above method to real analytic vector fields instead of holomorphic vector fields, we can extend Theorem A.4.13 to the following theorem.

Theorem A.4.21. Suppose a real analytic vector field $V$ satisfies the conditions (i) and (ii) in Theorem A.4.1 moreover, assume that all the eigenvalues satisfy (iii), i.e., are of Poincaré type, then the formal series of linearizing mapping convergent to a real analytic mapping

$$
\varphi:\left(\mathbb{R}^{n}, 0\right) \rightarrow\left(\mathbb{R}^{n}, 0\right)
$$

such that $\varphi_{*} V=V_{0}$.

Remark A.4.22. Actually, consider our operator $P$, the eigenvalues are of Poincaré type in attractor/repellent cases, it is not hard to have the linearization. For non-attractor cases, the eigenvalues are of Siegel type, there is a linearization theory for such kind of set of eigenvalues.

Now we are going to show some results about the linearization in the Siegel domain. In Siegel domain the denominators $\lambda_{j}-\langle\alpha, \lambda\rangle$ are not separated from the origin, then the inverse $\operatorname{ad}_{\Lambda}^{-1}$ is unbounded. However, $S_{F}$ is strongly contracting, equation A.2 can be solved with respect to $h$ by Newton-type iteration, while provided the small denominators $\left|\lambda_{j}-\langle\alpha, \lambda\rangle\right|$ do not approach the origin as fast as $|\alpha|$ goes to infinite. Such techniques is knowing as the KAM theory after A. Kolmogorov, V. Arnold and J. Moser. See Chapter 2 of [9] for detail.

Definition A.4.23. A tuple of complex numbers $\lambda \in \mathbb{C}^{n}$ from the Siegel domain $\mathfrak{S}$ is called Diophantine, if the small denominators decay no faster than polynomically with 
$\alpha$, i.e.,

$$
\exists C, N<+\infty, \quad \text { such that } \forall \alpha \in \mathbb{Z}_{+}^{n}, \quad\left|\lambda_{j}-\langle\alpha, \lambda\rangle\right|^{-1} \leq C|\alpha|^{N} .
$$

Otherwise the tuple (vector, collection) is called Liouvillean.

Theorem A.4.24. (Siegel theorem). If the linearization matrix $\Lambda$ of a holomorphic vector field is non-resonant of Siegel type and has Diophantine spectrum, then the vector field is holomorphically linearizable.

Definition A.4.25. A non-resonant collection $\lambda \in \mathbb{C}^{n}$ is said to satisfy the Brjuno condition, if the small denominators decrease sub-exponentially,

$$
\left|\lambda_{j}-\langle\alpha, \lambda\rangle\right|^{-1} \leq C e^{|\alpha|^{1-\varepsilon}}, \quad \text { as } \quad|\alpha| \rightarrow \infty,
$$

for some finite $C$ and positive $\varepsilon>0$.

Theorem A.4.26. (Brjuno theorem). A holomorphic vector field with non-resonant linearization matrix of Siegel type satisfying the Brjuno condition, is holomorphically linearizable.

Remark A.4.27. If the denominators $\left|\lambda_{j}-\langle\alpha, \lambda\rangle\right|$ accumulate to zero too fast, e.g., super-exponentially, then the corresponding germs are in general non-linearizable.

Remark A.4.28. For the non-attractor case in our problem, the tuple of eigenvalues are Diophantine. That is, the linearization theory in Theorem A.4.21 are not only true for attractor cases but also true for non-attractor cases.

Remark A.4.29. There are some linearization results for resonant vector fields [32], but in this case our generic condition for radial point is violated. 


\section{Appendix B}

\section{Ordinary differential equations}

In this chapter we will have a review of Kamatsu's results on hypoellipticity of ordinary operators in space of hyperfunctions, which we will need in Chapter 5.

\section{B.1 Hypoellipticity of ordinary differential operators}

Let $X$ be an open subset of $\mathbb{C}$ and $P(x, \partial)$ an ordinary differential operator of order $m$. Put $\mathscr{M}=\mathcal{D} / \mathcal{D} P$, and write

$$
P(x, \partial)=\sum_{k=0}^{m} a_{k}(x) \partial^{k} .
$$

Definition B.1.1. A point $x_{0}$ in $X$ is said to be a singular point of equation $P u=0$ if $a_{m}\left(x_{0}\right)=0$. Moreover, if $a_{m}\left(x_{0}\right) \neq 0$, then $x_{0}$ is said to be an ordinary point of $P u=0$.

In a neighborhood of an ordinary point $x_{0}$ in $X, \mathscr{M} \simeq \mathscr{O}_{X}^{\oplus m}$ as $\mathcal{D}$-modules, and $P u=0$ has $m$ linearly independent holomorphic solutions. However, in a neighborhood of a singular point $x_{0}$, the behavior of solutions are not easy to manipulate. For instance, while $L=\operatorname{Hom}_{\mathcal{D}_{X}}\left(\mathscr{M}, \mathscr{O}_{X}\right)$ is locally constant sheaf of rank $m$ on $X \backslash\left\{x_{0}\right\}$, a general algorithm for its monodromy is not known. But an algorithm is well known in the case of differential equations with regular singularities as follows.

Theorem B.1.2. A point $x_{0}$ is called a regular singular point of $P u=0$ if the following two conditions are equivalent:

(1) Let $r=\operatorname{ord}_{x=x_{0}} a_{m}(x)$. Then $\operatorname{ord}_{x=x_{0}} a_{j}(x) \geq r-(m-j)$ for all $j$, where $\operatorname{ord}_{x=x_{0}} f(x)$ denotes the order of zero of $f(x)$ at $x=x_{0}$. We set $\operatorname{ord}_{x=x_{0}} 0=\infty$.

(2) The equation $P(x, \partial) u=0$ has $m$ linearly independent solutions of the form

$$
\left(x-x_{0}\right)^{\lambda} \sum_{j=0}^{s} u_{j}(x)\left(\log \left(x-x_{0}\right)\right)^{j}
$$


for some $s \in \mathbb{Z}_{\geq 0}$ and $\lambda \in \mathbb{C}$, where $u_{j}(x)$ are holomorphic on a neighborhood of $x=x_{0}$.

Furthermore, a zero of $a_{m}(x)$ not satisfying the above conditions is called an irregular singular point.

We define a coherent filtration $F(\mathscr{M})$ of

$$
\mathscr{M}=\mathcal{D} / \mathcal{D} P=\mathcal{D} u \quad(u=1 \quad \bmod \mathcal{D} P)
$$

by

$$
F_{k}(\mathscr{M})=\sum_{j=0}^{m-1} F_{k}(\mathcal{D})(x \partial)^{j} u .
$$

Theorem B.1.3. Say $x$ is a regular singularity of $\mathscr{M}$ if and only if $\mathscr{M}$ admits a coherent filtration satisfying

$$
(x \partial) F_{k}(\mathscr{M}) \subset F_{k}(\mathscr{M}) \quad \text { or } \quad x \xi \operatorname{Gr}^{F}(\mathscr{M})=0,
$$

where $\mathrm{Gr}^{F}$ is the grade algebra associated with the filtration $F$.

Definition B.1.4. Consider a linear homogeneous ordinary differential equation in complex domain

$$
P(x, \partial) u(x)=\sum_{i=0}^{m} a_{i}(x) \frac{d^{i} u(x)}{d x^{i}}=0,
$$

with analytic coefficients. The equation $P u=0$ belongs to Fuchsian class if and only all its singular points on the Riemann sphere are regular singular points.

Lemma B.1.5. Let $T=z \frac{d}{d z}$, then

(i) We have

$$
T^{n}=z^{n} \frac{d^{n}}{d z^{n}}+\sum_{i=1}^{n} s_{i} z^{n-i} \frac{d^{n-i}}{d z^{n-i}}, \text { with } s_{i} \in \mathbb{Z}
$$

(ii) We have

$$
z^{n} \frac{d^{n}}{d z^{n}}=T^{n}+\sum_{j=1}^{n} d_{j} T^{n-j}, \text { with } d_{i} \in \mathbb{Z} .
$$

Let $\Omega$ be an open set in $\mathbb{R}$, consider a linear ordinary differential equation

$$
P u(x)=f(x)
$$

where $f(x) \in \mathscr{B}(\Omega)$, and the operator $P$ is of the form

$$
P=\sum_{i=0}^{m} a_{i}(x) \frac{d^{i}}{d x^{i}}
$$

where all the coefficients $a_{j}(x)$ are real analytic in $\Omega$ and $a_{m}(x) \not \equiv 0$. 
Theorem B.1.6. (Komatsu,[52]) Let $\Omega$ be an open subset in $\mathbb{R}$, then

$$
\operatorname{dim}\left(\mathscr{B}^{P}\right)=m+\sum_{x \in \Omega} \operatorname{ord}_{x} a_{m}(x)
$$

where $\operatorname{ord}_{x} a_{m}(x)$ is the order of zero at $x$ of the coefficient $a_{m}(x)$.

Theorem B.1.7. (Komatsu,[52]) Let $\Omega$ be an open subset in $\mathbb{R}$, then the following three conditions are equivalent:

(i) All hyperfunction solutions associated with the operator $P$ are real analytic in $\Omega$, i.e., $\mathscr{B}^{P}(\Omega) \subset \mathcal{A}(\Omega)$,

(ii) The leading coefficient $a_{m}(x)$ does not vanish for every $x \in \Omega$,

(iii) If $f(x)$ is real analytic in $\Omega$, so does $u(x)$.

Theorem B.1.7 gives the condition for $\mathcal{A}$-hypoellipticity of the operator $P$. Before we discuss the $\mathcal{D}^{\prime}$-hypoellipticity of the operator $P$, first we give two lemmas.

Lemma B.1.8. Let $\Omega$ be an open subset of $\mathbb{R}$, a hyperfunction $f(x) \in \mathscr{B}(\Omega)$ belongs to $\mathscr{D}^{\prime}(\Omega)$ if and only if a defining function $F(z)$ of $f$ satisfies the following estimate: for any compact set $K \subset \Omega$, there are $N \in \mathbb{N}, C>0$ and $\epsilon>0$ such that for any $0<|y|<\epsilon$, one has

$$
\sup \{|F(x+i y)| ; x \in K\} \leq \frac{C}{|y|^{N}} .
$$

If the above condition is satisfied, the distribution in $x, F(x+i y)$ and $F(x-i y)$ for $y>0$, converge in the topology of $\mathscr{D}^{\prime}(\Omega)$ as $y \rightarrow 0$. Then one has a distribution identity:

$$
f(x)=\lim _{y \rightarrow 0} F(x+i y)-\lim _{y \rightarrow 0} F(x-i y) .
$$

Komatsu [52] also discussed the conditions of making a hyperfunction belongs to $L_{l o c}^{p}(\Omega)$, ultradistribution $\mathscr{D}^{\prime(s)}$ of class $(\mathrm{s})$, and the local Besov space $B_{p, q, l o c}^{\sigma}(\Omega)$ for $-\infty<\sigma<\infty, 1 \leq p, q \leq \infty$.

Lemma B.1.9. Let $\Omega$ be an open subset of $\mathbb{R}^{n}$, a hyperfunction $f(x)=\in \mathscr{B}(\Omega)$ belongs to $\mathscr{D}^{\prime}(\Omega)$ if and only if there is a representation of $f$,

$$
f(x)=\sum_{j=1}^{k} F_{j}(z), \quad F_{j}(z) \in \mathscr{O}\left(\Omega+i \Gamma_{j} 0\right), j=1, \cdots, k
$$

satisfies the following estimate: for every compact set $K \subset \Omega$ and for every proper subcone $\triangle_{j} \subset \Gamma_{j}$, there are $N \in \mathbb{N}, C>0$ and $\epsilon>0$ such that for each $j=1, \cdots, k$, one has

$$
\sup \left\{\left|F_{j}(z)\right| ; x \in K\right\} \leq \frac{C}{|y|^{N}} \quad \text { for } \quad z \in K+i\left(\triangle_{j} \cap\{|y|<\epsilon\}\right) 0 .
$$


Then for any fixed $y \in \Gamma$, the real analytic function $F_{j}(x+i \epsilon y)$ in $x$ is convergent in $\mathscr{D}^{\prime}(\Omega)$ as $\epsilon \rightarrow 0$, the distribution $f(x)$ obtained as the limit is nothing but

$$
\sum_{j=1}^{k} F(x+i \Gamma 0)
$$

when regarded as a hyperfunction.

Theorem B.1.10. (Komatsu, [52]) Consider an ordinal differential equation in $\Omega \subset \mathbb{R}$

$$
P\left(x, \frac{d}{d x}\right) u(x)=\sum_{j=0}^{m} a_{j}(x) \frac{d^{j}}{d x^{j}} u(x)=f(x),
$$

where all the coefficients $a_{j}(x), j=0, \cdots, m$ are real analytic. If all the singular points in $\Omega$ are regular, and if $f \in \mathscr{D}^{\prime}(\Omega)$, then the hyperfunction solutions $u \in \mathscr{D}^{\prime}(\Omega)$.

Moreover, if there is an irregular singular point, then one can construct a hyperfunction solution $u$ that is not a distribution.

Proof. Without loss of generality, suppose the origin is the unique regular singular point in $\Omega$. And let $U(z) \in V \backslash \Omega$ and $F(z) \in V \backslash \Omega$ be the defining function of $u$ and $f$ respectively, where $V \in \mathbb{C}$ is the complex neighborhood of $\Omega$. Then our equation can be naturally written as

$$
P\left(z, \frac{d}{d z}\right) U(z)=\sum_{j=0}^{m} a_{j}(z) \frac{d^{j}}{d z^{j}} U(z)=F(z),
$$

Use the Lemma B.1.5, we have

$T^{n} U(z)+b_{n-1}(z) T^{n-1} U(z)+b_{n-2}(z) T^{n-2} U(z)+\cdots+b_{1}(z) T U(z)+b_{0}(z) U(z)=F(z)$, where $b_{k}, k=0, \cdots, n-1$, are holomorphic on $V$. Let

$$
W(z)=\left(U(z),\left(z \frac{d}{d z}\right) U(z), \cdots,\left(z \frac{d}{d z}\right)^{m-1} U(z)\right)^{T},
$$

then

$$
\left(z \frac{d}{d z}+B(z)\right) W(z)=F(z)
$$

where

$$
B=\left(\begin{array}{cccccc}
0 & -1 & 0 & \cdots & 0 & 0 \\
0 & 0 & -1 & \cdots & 0 & 0 \\
\vdots & \vdots & \vdots & \ddots & \vdots & \vdots \\
0 & 0 & 0 & \cdots & -1 & 0 \\
0 & 0 & 0 & \cdots & 0 & -1 \\
b_{0} & b_{1} & b_{2} & \cdots & b_{n-2} & b_{n-1}
\end{array}\right)
$$

which is a matrix whose elements are bounded near 0 (we can assume this by shrinking 
$V)$ and $F(z)$ satisfies

$$
|F(x+i y)| \leq \frac{C}{|y|^{N}}
$$

for some constants $C$ and $N$.

Choose $t=\log \left(\frac{ \pm i}{z}\right)$ and the equation is transformed into

$$
\left(c \frac{d}{d t}+\tilde{B}(t)\right) \tilde{W}(t)=\tilde{F}(t)
$$

where $c$ is a constant depending only on $\tilde{B}(t)$, which does not vanish. And one times $\frac{1}{c}$ on both sides, still keeps the same notations, one has the inhomogeneous equation of order one with variable coefficients

$$
\left(\frac{d}{d t}+\tilde{B}(t)\right) \tilde{W}(t)=\tilde{F}(t)
$$

one have the solution of the form

$$
\tilde{V}(t)=e^{-b(t)}\left(\int \tilde{F}(t) e^{b(t)} d t+\kappa\right),
$$

where $\kappa$ is a constant of integration and

$$
b(t)=\int e^{\tilde{B}(t)} d t
$$

Since $\tilde{B}(t)$ is bounded, then there exist two positive numbers $b_{1}$ and $b_{2}$ such that $b_{1}<$ $b(t)<b_{2}$.

$$
\tilde{V}(t)=e^{-b(t)}\left(\int \tilde{F}(t) e^{b(t)} d t+\kappa\right)<e^{-b_{1}}\left(e^{b_{2}} \int \tilde{F}(t) d t+\kappa\right),
$$

And one can find the estimate

$$
\begin{aligned}
|V(x+i y)| & =|\tilde{W}(t)| \\
& \leq e^{-b_{1}}\left(e^{b_{2}} \int|\tilde{F}(t)| d t+|\kappa|\right) \\
& =e^{-b_{1}}\left(e^{b_{2}} \int|F(z)| d z+|\kappa|\right) \\
& \leq \frac{C^{\prime}}{|y|^{N^{\prime}}}
\end{aligned}
$$

for some constants $C^{\prime}$ and $N^{\prime}$.

Suppose $\sigma>1$ be the irregularity of the origin, i.e., the maximal gradient of the highest convex polygon below the points $\left(j\right.$, ord $\left._{x=0} a_{j}(x)\right), j=0, \cdots, m$. For $\sigma \leq 1$, the singularity is called determined singularity and for $\sigma>1$ the singularity is called non-determined singularity.

Then one can find on each sector with angle less then $\frac{\pi}{\sigma-1}$ and summit at 0 , a 
holomorphic solution $U(z)$ of $P\left(z, \frac{d}{d z}\right) U(z)=0$ which has the asymptotic expansion

$$
U(z) \sim e^{\frac{\lambda}{z^{\sigma-1}}+\cdots} z^{\rho} p(z, \log z),
$$

where $\lambda$ is non-zero constant and $p$ is a polynomial on $\log z$ whose coefficients are formal power series of $z^{r}$ for some $r>0$.

If one choose the sector in the upper or the lower half plane due to the argument of $\lambda$, the solution satisfies the estimate

$$
\sup _{x \in K}|U(x+i y)| \geq C e^{\left(\frac{L}{|y|}\right)^{\sigma-1}}
$$

on the half plane.

Remark B.1.11. In Theorem B.1.10, if we consider the homogeneous case $P u=0$ and all the singular points of $P$ are regular, then the collection of such solutions belongs to $\mathscr{B}_{\text {Nils }}(U)$, where $\mathscr{B}_{\text {Nils }}$ is the sheaf of hyperfunctions in the Nilsson class [61], which is a subsheaf of $\mathscr{B}$. For more information of hyperfunctions in the Nilsson class, please check part II of F. Pham [61].

Example B.1.12. Consider the Euler operator

$$
P=x \frac{d}{d x}-\alpha
$$

one can fine the radial point $(0, \pm 1)$. The irregularity of $P$ at the singular point $x=$ 0 is 1 , that is 0 is of determined singularity. The solution space is of 2 dimensions. According to the Theorem B.1.10, if $P u \in \mathscr{D}^{\prime}$, then the hyperfunction solutions $u \in \mathscr{D}^{\prime}$.

In fact, one can choose the basis of solution space $\mathscr{B}^{P}$ as:

(1) $\alpha=0,1,2, \cdots$, choose $x^{\alpha}, x_{+}^{\alpha}$,

(2) $\alpha=-1,-2, \cdots$, choose $\delta^{-\alpha-1}, p \cdot v \cdot\left(x^{\alpha}\right)$,

(3) $\alpha \notin \mathbb{Z}$, choose $x_{-}^{\alpha}, x_{+}^{\alpha}$.

Now we have another example of non-determined singularity in one dimension.

\section{Example B.1.13.}

$$
P=x^{2} \frac{d}{d x}-c
$$

where $c$ is some constant. The irregularity of $P$ at $x=0$ is 2 . The solution space is of 3 dimensions.

Actually, one can choose the basis of solution space $\mathscr{B}^{P}$ as following. Define a smooth function equal to $e^{-\frac{1}{x}}$ when $x \neq 0$ and equal to 0 when $x=0$. And consider two hyperfunctions which are not distributions $e^{-\frac{1}{x+i 0}}$ and $e^{-\frac{1}{x+i 0}}-e^{-\frac{1}{x-i 0}}$.

That is, if $P u \in \mathscr{D}^{\prime}$, then there might exists hyperfunction solutions $u$ which are not distributions. 


\section{Appendix C}

\section{Fourier hyperfunctions}

In this chapter we have a review of Fourier hyperfunctions. The main references are Kaneko [34] and Graf [20]. The former is one of the best monograph to study the hyperfunction theory, and the latter one gives a delicate and very detailed description on the various integral transforms of hyperfunctions of one variable.

\section{C.1 Fourier transformations}

Proposition C.1.1. For $\alpha=\left(\alpha_{1}, \cdots, \alpha_{n}\right) \in \mathbb{Z}^{n}$, let $u(x)=\sum_{a_{\alpha}, \alpha \in \mathbb{Z}^{n}} a_{\alpha} e^{2 \pi \sqrt{-1}\langle x, \alpha\rangle}$, where $x \in \mathbb{R}^{n}$. Suppose for any arbitrary $\epsilon>0$ there exists a constant $C_{\epsilon}$ such that $\left|a_{\alpha}\right| \leq C_{\epsilon} e^{\epsilon|\alpha|}$ holds for each $\alpha$, where $|\alpha|=\sum_{j=1}^{n}\left|a_{j}\right|$. Then $u(x)$ is a well-defined hyperfunction. If $\left|a_{\alpha}\right| \leq C|\alpha|^{N}$ for some $N>0$ and $C>0$, then $u(x)$ is a distribution.

Proposition C.1.2. Let $f(y)$ be a locally Lebesgue integrable function on $\mathbb{R}^{n}$ such that for an arbitrary $\epsilon>0$ there exists $C_{\epsilon}$ with the property $|f(y)| \leq C_{\epsilon} e^{\epsilon|y|}$ almost everywhere. Then the Fourier transform of $f(y), u(x)=\int_{\mathbb{R}^{n}} f(y) e^{2 \pi \sqrt{-1}\langle x, y\rangle} d y$ is a well-defined hyperfunction.

Now let us examine the singular spectrum of the hyperfunction

$$
u(x)=\int_{\mathbb{R}^{n}} f(y) e^{2 \pi \sqrt{-1}\langle x, y\rangle} d y,
$$

supposing some additional information on $\operatorname{supp} f$.

Definition C.1.3. For a subset $G$ of $\mathbb{R}^{n}$, define

$$
\begin{gathered}
G \infty=\left\{y \in \mathbb{R}^{n}-\{0\} \mid \text { for an arbitrary } N>0 \text { and } \epsilon>0,\right. \\
\left.G \cap\left\{y^{\prime}|| y^{\prime} \mid \geq N \text { and }\left|\frac{y^{\prime}}{\left|y^{\prime}\right|}-\frac{y}{|y|}\right|<\epsilon\right\} \neq \emptyset\right\} .
\end{gathered}
$$

Lemma C.1.4. Let $G$ be a subset of $\mathbb{R}^{n}$. Let $\mathscr{G}$ be the collection of $\left\{G^{\prime} \subset \mathbb{R}^{n} \mid G^{\prime}\right.$ is a cloesed cone such that $G^{\prime} \supset G+a$ for some $\left.a\right\}$. 
Then $G \infty=\bigcap_{G^{\prime} \in \mathscr{G}} G^{\prime}$ holds.

Proposition C.1.5. Let $f(x)$ be the same as in previous proposition and let $G=\operatorname{supp} f$. Then the singular spectrum of the hyperfuction

$$
u(x)=\int_{\mathbb{R}^{n}} f(y) e^{2 \pi \sqrt{-1}\langle x, y\rangle} d y,
$$

is contained in $\{(x, \sqrt{-1} \xi \infty) \mid \xi \in G \infty\}$.

Proposition C.1.6. Denote

$$
D^{\alpha}=D_{1}^{\alpha_{1}} \cdots D_{n}^{\alpha_{n}}, \quad D_{j}=\frac{1}{\sqrt{-1}} \frac{\partial}{\partial x_{j}}
$$

assume $\mathscr{F}$ be the action of Fourier transformation, we have

(a) $\mathscr{F}\left(D^{\alpha} f\right)=\xi^{\alpha} \mathscr{F} f$. More generally, for a local operator $J(D)$ with constant coefficients, $\mathscr{F}(J(D) f)=J(D) \mathscr{F} f$.

(b) $\mathscr{F}\left(x^{\alpha} f\right)=\left(-D_{\xi}\right) \mathscr{F} f$.

(c) $\mathscr{F}(f(a x+b))=\frac{1}{|a|} e^{\sqrt{-1} \frac{b}{a} \xi} \mathscr{F}\left(\frac{\xi}{a}\right)$, in particular, $\mathscr{F}(f(x-a))=e^{-\sqrt{-1} a \xi} \mathscr{F} f$, $\mathscr{F}(f(a x))=\frac{1}{|a|} \mathscr{F}\left(f\left(\frac{\xi}{a}\right)\right)$, and $\mathscr{F}(f(-x))=\mathscr{F}(f(-\xi))$.

(d) $\mathscr{F}(f * g)=\mathscr{F}(f) \cdot \mathscr{F}(g)$.

(e) $\mathscr{F}(\overline{f(x)})=\overline{\mathscr{F}(f(-\xi))}, \mathscr{F}(\overline{f(-x)})=\overline{\mathscr{F}(f(\xi))}$. That is, the Fourier transformation of a real and even hyperfunction is a real hyperfunction. The Fourier transformation of a real and odd hyperfunction is an imaginary hyperfunction.

\section{C.2 Fourier transformation of hyperfunctions with compact support}

Consider a hyperfunction $f(x)$ on $\mathbb{R}^{n}$ with compact support $K$, then the integral

$$
\hat{f}(\zeta)=\int_{\mathbb{R}^{n}} e^{-i x \zeta} f(x) d x
$$

is well-defined for every $\zeta \in \mathbb{C}^{n}$. That is the Fourier transformation of $f(x)$ can be regarded as a function of complex variables. More precisely, consider the boundary values representation

$$
f(x)=\sum_{j=1}^{N} F_{j}\left(x+i \Gamma_{j} 0\right),
$$


and the Fourier transformation of $f$ can be defined as

$$
\hat{f}(\zeta)=\sum_{j=1}^{N} \int_{D_{j}} e^{-i z \zeta} F_{j}(z) d z
$$

Here, $D_{j}$ denotes an integral path obtained by fixing the boundary of real neighborhood $D$ of $K$ and deforming its interior into the inside of the infinitesimal wedge $\mathbb{R}^{n}+$ $\sqrt{-1} \Gamma_{j} 0$ which is the domain of analyticity of $F_{j}(z)$.

Similar as Paley-Wiener theorem, we have

Theorem C.2.1. (Paley-Wiener-Ehrenpreis theorem) The Fourier transformation of a hyperfunction $f(x)$ with support contained in a compact set $K$ is an entire function of $\zeta$, and for any $\epsilon$, there exists $C_{\varepsilon}>0$ such that

$$
|\hat{f}(\zeta)| \leq C_{\epsilon} e^{\epsilon|\zeta|+H_{K}(\operatorname{Im} \zeta)}
$$

Here,

$$
H_{K}(\eta)=\sup _{x \in K} x \eta
$$

is called the supporting function of $K$. Conversely, an entire function which satisfies the above conditions is necessarily the Fourier transformation of a unique hyperfunction whose support is contained in the convex hull of $K$.

\section{C.3 Fourier transformation of exponentially decreasing hyperfunctions}

Definition C.3.1. Let $\delta$ be a positive constant and let $\mathbb{R}^{n}+i I$ be a tubular domain. A function $F(z) \in \mathscr{O}\left(\mathbb{R}^{n}+i I\right)$ is said to decrease exponentially with type $-\delta$ (along the real axis), if for every compact subset $K \Subset I$ and every positive $\epsilon$, there exists $C_{K, \epsilon}>0$ such that

$$
|F(z)| \leq C_{K, \epsilon} e^{-(\delta-\epsilon)|\operatorname{Re} z|}
$$

uniformly for $z \in \mathbb{R}^{n}+i K$. The set of all such functions is denoted by $\tilde{\mathscr{O}}^{-\delta}\left(\mathbb{D}^{n}+i I\right)$. Here, $\mathbb{D}^{n}$ is the compactification $\mathbb{R}^{n} \cup S_{\infty}^{n-1}$ of $\mathbb{R}^{n}$, where $S_{\infty}^{n-1}$ is an $(n-1)$-dimensional sphere at infinity. When $x$ is a vector in $\mathbb{R}^{n} \backslash\{0\}$, we denote by $x \infty$ the point on $S^{n-1}$ which is represented by $x$, where we identify $S^{n-1}$ with $\mathbb{R}^{n} \backslash\{0\} / \mathbb{R}_{+}$. The space $\mathbb{D}^{n}$ is given the natural topology, that is:

(1) If a point $x$ of $\mathbb{D}^{n}$ belongs to $\mathbb{R}^{n}$, a fundamental system of neighborhoods of $x$ is the set of all open balls containing the point $x$.

(2) If a point $x \in \mathbb{D}^{n}$ belongs to $S_{\infty}^{n-1}$, a fundamental system of neighborhoods of $x(=y \infty)$ is given by the following family

$$
U_{\tilde{\Delta}, A}(y \infty)=\left\{x \in \mathbb{R}^{n} ; \frac{x}{|x|} \in \tilde{\Delta},|x|>A\right\} \cup\{a \infty ; a \in \tilde{\triangle}\},
$$


where $\tilde{\triangle}$ is a neighborhood of $y$ in $S^{n-1}$.

Also, define

$$
\mathcal{A}_{*}=\underset{I \ni 0}{\lim } \underset{\delta \rightarrow 0}{\lim } \tilde{\mathscr{O}}^{-\delta}\left(\mathbb{D}^{n}+i I\right),
$$

and call it the space of rapidly decreasing real analytic functions.

The estimate obtained by setting $\delta=0$ from above

$$
|F(z)| \leq C_{k, \epsilon} e^{\epsilon|\operatorname{Re} z|}
$$

is called an infra-exponential type along the real axis, or simply slowly increasing, and the totality of functions satisfying this estimate is denoted by $\tilde{\mathscr{O}}\left(\mathbb{D}^{n}+i I\right)$.

Remark C.3.2. Notice that the notations are different from Schwartz distribution. Here we follow Sato's language to use the terms rapidly decreasing for exponential decay and slowing increasing for infra-exponential growth, and keep the term exponential decay if the exponential decay rate is globally fixed on the domain.

Use the multi-index notations $|\alpha|=\alpha_{1}+\alpha_{2}+\cdots+\alpha_{n}$, $\partial^{\alpha}=\partial_{1}^{\alpha_{1}} \cdots \partial_{n}^{\alpha_{n}}$ for $\alpha=\left(\alpha_{1}, \cdots, \alpha_{n}\right) \in \mathbb{N}_{0}^{n}$, where $\mathbb{N}_{0}$ is the set of nonnegative integers, we have

Definition C.3.3. A real valued function $\varphi$ is in Sato's space $\mathcal{F}$ if $\varphi \in C^{\infty}\left(\mathbb{R}^{n}\right)$ and if there exist $h, k>0$ satisfying

$$
|\varphi|_{k, h}=\sup _{\alpha, x} \frac{\left|\partial^{\alpha} \varphi(x)\right|}{h^{|\alpha|} \alpha !} e^{k|x|}<\infty .
$$

Moreover, we have the following Pringsheim's characterization of real analytic functions:

Theorem C.3.4. A smooth function $f$ on $X$ is analytic if and only if for every compact subset $K \Subset X$ there are positive constant $C, r=\frac{1}{h}$ satisfying

$$
\sup _{\alpha, x} \frac{\left|\partial^{\alpha} f(x)\right| r^{\alpha}}{\alpha !}=\sup _{\alpha, x} \frac{\left|\partial^{\alpha} f(x)\right|}{\alpha ! h^{|\alpha|}}<\infty .
$$

The above definition of Sato's space induced by D. Kim and others [11] is isomorphic to the space $\mathcal{A}_{*}$ introduced by Sato and Kawai. Denote by $\mathcal{F}^{\prime}$ the strong dual space of $\mathcal{F}$ and call its elements Fourier hyperfunctions.

Proposition C.3.5. ([11]) For the Sato's space $\mathcal{F}$, the following statements are equivalent,

(1) $\varphi \in \mathcal{F}$.

(2) There exist constants $h, k>0$ such that

$$
\sup _{x}|\varphi(x)| e^{k|x|}<\infty, \quad \sup _{x}|\hat{\varphi}(\xi)| e^{h|\xi|}<\infty .
$$


Remark C.3.6. The Schwartz space consists of all locally integrable function $\varphi$ such that $\varphi$ itself and its Fourier transform $\hat{\varphi}$ are both rapidly decreasing, while the Sato's space $\mathcal{F}$ consists of all locally integrable function $\varphi$ such that $\varphi$ itself and its Fourier transform $\hat{\varphi}$ are both exponentially decreasing.

Sato [64] introduced the notation of Fourier hyperfunctions that grow at infinity no faster than any linear exponential. In [46] Kawai claimed that the space of Fourier hyperfunctions on $\mathbb{R}^{n}$ can be identified with the continuous dual of a suitable test function space. Actually we can see from Proposition C.3.5 that the Sato's space of Fourier hyperfunctions is coinciding with the Gelfand-Shilov space $S_{1}^{1}\left(\mathbb{R}^{k}\right)$. A. G. Smirnov [70] introduced a way to construct the Fourier transoframtion of general hyperfunctions with no growth restrictions imposed.

Proposition C.3.7. We have the following properties,

(a) The image of the Fourier transformation of $\tilde{\mathscr{O}}^{-\delta}\left(\mathbb{D}^{n}+i\{|y|<\gamma\}\right)$ coincides with $\tilde{\mathscr{O}}^{-\gamma}\left(\mathbb{D}^{n}+i\{|y|<\delta\}\right)$.

(b) The Fourier transformation is a automorphism of $\mathcal{A}_{*}$.

Assume that the tubular domains $\mathbb{R}^{n}+i I_{j}$ are also infinitesimal wedge of type $\mathbb{R}^{n}+i \Gamma_{j} 0$.

Definition C.3.8. A hyperfunction of representation $f(x)=\sum_{j=1}^{N} F_{j}\left(x+i \Gamma_{j} 0\right)$ with defining functions $F_{j}(z) \in \tilde{\mathscr{O}}^{-\delta}\left(\mathbb{D}^{n}+i I\right)$ is called Fourier hyperfunction decreasing exponentially with type $-\delta$, or simply, an exponentially decreasing hyperfunction. The set of all such hyperfunctions is denoted by $\mathscr{Q}^{-\delta}$.

Proposition C.3.9. ([34], Theorem 8.2.6) The space $\mathscr{Q}^{-\delta}$ of exponentially decreasing hyperfunctions of type $-\delta$ and the space $\tilde{\mathscr{O}}\left(\mathbb{D}^{n}+i\{|\eta|<\delta\}\right)$ of slowly increasing holomorphic functions are in one-to-one correspondence via Fourier transformation.

\section{C.4 Fourier transformation of slowly increasing hyperfunctions}

Definition C.4.1. A hyperfunction of representation $f(x)=\sum_{j=1}^{N} F_{j}\left(x+i \Gamma_{j} 0\right)$ with slowly increasing holomorphic functions $F_{j}(z) \in \tilde{\mathscr{O}}\left(\mathbb{D}^{n}+i I_{j}\right)$ on tubular domains $\mathbb{D}^{n}+i I_{j}$ of type $\mathbb{D}^{n}+i \Gamma_{j} 0$ as defining functions, is called slowly increasing Fourier hyperfunction, or simply, slowly increasing hyperfunction. The set of all such hyperfunctions is denoted by $\mathscr{Q}$.

Proposition C.4.2. ([34], Theorem 8.3.4) The Fourier transformation is an automorphism of $\mathscr{Q}$.

Proposition C.4.3. ([34], Proposition 8.3.2)Let $F\left(x+i \Gamma_{j}\right)$ be a slowly increasing hyperfunction which decreases exponentially outside a closed convex proper cone $\Delta^{\circ}$. 
More precisely, there is a infinitesimal wedge $\mathbb{R}^{n}+i I$ of type $\mathbb{R}^{n}+i \Gamma 0$ such that when restricting $\operatorname{Re} z$ outside any cone containing $\Delta^{\circ}$ as a proper subcone, $F(z)$ satisfies the estimate, for a suitable $\delta>0$

$$
|F(z)|=O\left(e^{-\delta|\operatorname{Re} z|}\right)
$$

locally uniformly for $y \in I$. Set

$$
G(\zeta)=\int_{\operatorname{Im} z=y} e^{-i z \zeta} F(z) d x
$$

for any $y \in I$. Then it converges locally uniformly in $\zeta$ running over a tubular domain $\mathbb{R}^{n}-i J$ of type $\mathbb{R}^{n}-i \Delta 0$, and $G(\zeta) \in \tilde{\mathscr{O}}\left(\mathbb{D}^{n}-i J\right)$. Moreover, $G(\zeta)$ decreases exponentially outside $\Gamma^{\circ}$. Hence

$$
\mathscr{F}(F(x+i \Gamma 0))=G(\zeta-i \Delta 0)
$$

is well defined as a slowly increasing hyperfunction, while $\mathscr{F}$ is a action of Fourier transformation.

\section{C.5 Embeddings, localization, topology and duality}

Theorem C.5.1. One has the embedding

$$
\mathscr{B}_{*}\left(\mathbb{R}^{n}\right) \hookrightarrow \mathscr{Q}^{-\delta} \hookrightarrow \mathscr{Q} .
$$

Here, $\mathscr{B}_{*}\left(\mathbb{R}^{n}\right)$ is the space of hyperfunctions with compact support. In addition, the definition of Fourier transformation for these spaces is consistent with embeddings.

Theorem C.5.2. One has the embedding

$$
\mathcal{A}_{*} \hookrightarrow \overline{\mathcal{A}} \hookrightarrow \mathscr{Q} .
$$

Here, $\mathcal{A}_{*}$ is the space of rapidly decreasing real analytic functions, and $\overline{\mathcal{A}}$ is the space of slowly increasing real analytic functions

$$
\overline{\mathcal{A}}=\underset{\delta \rightarrow 0}{\lim _{\delta \rightarrow 0}} \tilde{\mathscr{O}}\left(\mathbb{D}^{n}+i\{|y|<\delta\}\right) .
$$

Remark C.5.3. Notice that $\mathscr{Q}$ can be naturally mapping into $\mathscr{B}$, and the mapping is surjective. Moreover, the mapping $\mathscr{Q}^{-\delta} \rightarrow \mathscr{B}\left(\mathbb{R}^{n}\right)$ is also surjective.

Proposition C.5.4. For every slowly increasing hyperfunction $f(x)$ and exponential decreasing hyperfunction $g(x)$, the convolution

$$
f * g=\int_{\mathbb{R}^{n}} f(x-y) g(y) d y
$$

can be defined, and it is a slowly increasing hyperfunction. Further more, the product 
of a slowly increasing function and a slowly increasing real analytic function is well defined as a slowly increasing hyperfunction. Moreover, we have the relation:

$$
\widehat{f * g}=\widehat{f} \cdot \widehat{g} .
$$

Proposition C.5.5. Every local operator $J(D)=\sum b_{\alpha} D^{\alpha}$ with constant coefficients acts on every Fourier hyperfunction $f(x)$, i.e. $J(D): \mathscr{Q} \rightarrow \mathscr{Q}\left(\right.$ or $\left.\mathscr{Q}^{-\delta} \rightarrow \mathscr{Q}^{-\delta}\right)$. Moreover,

$$
\widehat{J(D) f(x)}=J(\xi) \cdot \widehat{f}
$$

holds. If $g(x)$ is an exponentially decreasing hyperfunction, then

$$
J(D)(f * g)=J(D) f * g=f * J(D) g
$$

also holds.

Now we are going to discuss the localizability of Fourier hyperfunctions. Fourier hyperfunctions can also be defined cohomologically by using $\tilde{\mathscr{O}}$. One can recognize it as a sheaf of a class of holomorphic functions with growth order as $x \rightarrow \infty$ such as $\tilde{\mathscr{O}}$.

Consider the compactification of $\mathbb{R}^{n}$

$$
\mathbb{D}=\mathbb{R}^{n} \sqcup S_{\infty}^{n-1}
$$

by adding points at infinite at all directions.

Take a fundamental system of neighborhoods of a point at infinity $a \infty$ as the following family

$$
U_{\Delta, A}(a \infty)=\left\{x \in \mathbb{R}^{n} ; x /|x| \in \Delta,|x|>A\right\} \sqcup\{x \infty ; x \in A\},
$$

where $\Delta$ is a neighborhood of $a$ in $S^{n}$. One has $\mathbb{C}$ is embedded into $\mathbb{D}^{n}+i \mathbb{R}^{n}$. Denote the closure of a set $V$ taken in $\mathbb{D}^{n}$ or $\mathbb{D}^{n}+i \mathbb{R}^{n}$ as $\overline{\bar{V}}$, and the one taken in $\mathbb{R}^{n}$ and $\mathbb{C}^{n}$ is $\bar{V}$. Then $\mathbb{D}^{n}+i \mathbb{R}^{n}$ can be written as $\overline{\overline{\mathbb{C}^{n}}}$.

The function space $\tilde{\mathscr{O}}$ can be naturally be regarded as the space of global sections of a sheaf on $\mathbb{D}^{n}+i \mathbb{R}^{n}$.

Proposition C.5.6. $\mathbb{D}^{n} \subset \mathbb{D}^{n}+i \mathbb{R}^{n}$ is purely $n$-codimensional with respect to the sheaf $\tilde{\mathscr{O}} 1$ The derived sheaf $\mathscr{Q}=\mathscr{H}_{\mathbb{D}^{n}}^{n}(\tilde{\mathscr{O}})$ is called the sheaf of slowly increasing Fourier hyperfunctions. Also $\left.\mathscr{Q}\right|_{\mathbb{R}^{n}}=\mathscr{B}$.

Proposition C.5.7. $Q$ is a flabby sheaf on $\mathbb{D}^{n}$.

One can define the sheaf consisting of exponentially decreasing hyperfunctions of all types as

$$
\mathscr{Q}_{*} \triangleq \lim _{\delta \rightarrow 0} \mathscr{Q}^{-\delta}
$$

\footnotetext{
${ }^{1}$ A closed set $A$ in a topological space $X$ is purely $n$-codimensional with respect to the sheaf $\mathscr{F}$ over $X$ if $H_{A}^{j}(\mathscr{F})=0$ for $j \neq n$. For instance, the Euclidean space $\mathbb{R}^{n}$ in $\mathbb{C}^{n}$ is purely $n$-codimensional with respect to the sheaf $\mathscr{O}$.
} 
Since $\left.\mathscr{Q}_{*}\right|_{\mathbb{R}^{n}}=\mathscr{B}$, the quotient sheaf $\mathscr{Q} / \mathscr{Q}_{*}$ may be regarded as the one extracting only growth order from Fourier hyperfunctions.

For $f(x) \in \mathscr{Q}\left(\mathbb{D}^{n}\right)$, define

$$
\operatorname{supp}_{\infty} f \triangleq \operatorname{supp}\left\{f(x) \bmod \mathscr{Q}_{*}\right\}
$$

and call it the essential support of $f$.

The relationship between growth order and singular spectrum is

Theorem C.5.8. For $f(x) \in \mathscr{Q}\left(\mathbb{D}^{n}\right)$, then the following statements are equivalent:

(i) S. S. $f(x) \subset \mathbb{D}^{n}+i \Gamma^{\circ} d x \infty$.

(ii) $\operatorname{supp}_{\infty} \hat{f}(\xi) \subset \Gamma^{\circ}$.

Corollary C.5.9. A hyperfunction $f(x) \in \mathscr{B}\left(\mathbb{R}^{n}\right)$ is micro-analytic at $(x, \sqrt{-1} \xi d x \infty)$ if and only there exist a conical neighborhood $\Gamma^{\circ}$ of $\xi$ and a Fourier hyperfunction $g$ such that $\operatorname{supp}_{\infty} \hat{g}(\xi) \cap \overline{\overline{\Gamma^{\circ}}}=\emptyset$ and that the difference $f(x)-g(x)$ is real analytic on a neighborhood of $x$.

Proposition C.5.10. ([34], Chapter 8, Page 392) We have the embedding

$$
\mathscr{B}_{*}\left(\mathbb{R}^{n}\right) \hookrightarrow \mathscr{Q} \rightarrow \mathscr{B}\left(\mathbb{R}^{n}\right)
$$

For any $f \in \mathscr{B}\left(\mathbb{R}^{n}\right)$, there exists a set of slowly increasing functions among defining functions of $f$ in the sense of hyperfunctions.

In Kaneko's book [34], he introduce a topology into the hyperfunction space and make it to be a Fréchet space (also a Montel space 2). By giving suitable topologies, he shows that $\mathcal{A}_{*}\left(\mathbb{D}^{n}\right)$ and $\mathscr{Q}\left(\mathbb{D}^{n}\right)$ are topologically dual to each other. Such duality can be extended to the duality between the space $\mathcal{A}_{*}(K)$ and the $\mathscr{Q}(K)$, while the former is the space of all rapidly decreasing real analytic functions defined on a neighborhood of compact subset $K$ of $\mathbb{D}^{n}$, and the latter is the the space of all slowly increasing hyperfunctions with support contained in $K$. The above duality includes the duality between $\mathscr{B}(K)$ and $\mathcal{A}(K)$. In Morimoto [59], we see the duality of $\mathscr{B}(K)$ and $\mathcal{A}(K)$, while $\mathscr{B}(K)$ is a Fréchet-Schwartz space, and $\mathcal{A}(K)$ is a dual Fréchet-Schwartz space.

\footnotetext{
${ }^{2} \mathrm{~A}$ Montel space is a barrelled topological vector space where every closed and bounded set is compact.
} 


\section{Appendix D}

\section{Fourier-Bros-Iagolnitzer transform}

\section{D.1 Fourier-Bros-Iagolnitzer transform}

Definition D.1.1. The standard Fourier-Bros-Iagolnitzer (abbr. FBI) transform is defined as

$$
T_{h}: \mathscr{S}^{\prime}\left(\mathbb{R}^{n}\right) \rightarrow \mathscr{S}^{\prime}\left(\mathbb{R}^{2 n}\right) \cap C^{\infty}\left(\mathbb{R}^{2 n}\right)
$$

by

$$
T_{h} u(x, \xi)=2^{-\frac{n}{2}}(\pi h)^{-\frac{3 n}{4}} \int e^{\frac{\sqrt{-1}}{h}(x-y) \xi-\frac{1}{2 h}(x-y)^{2}} u(y) d y,
$$

which can be interpreted as the Fourier transform modified by a Gaussian distribution. The Gaussian distribution localize in position and the Fourier transform localize in frequency, hence $T_{h} u$ describes the microlocal property of $u$.

Proposition D.1.2. Here we list some rudimentary properties of the FBI transform:

(1) If $u \in \mathscr{S}^{\prime}\left(\mathbb{R}^{n}\right)$, then $e^{\frac{\xi^{2}}{h}} T_{h} u(x, \xi)$ is a holomorphic function of $z=x-\sqrt{-1} \xi$.

(2) If $u \in L^{2}\left(\mathbb{R}^{n}\right)$, then $T u \in L^{2}\left(\mathbb{R}^{n}\right)$ and $\|T u\|_{L^{2}\left(\mathbb{R}^{n}\right)}=\|u\|_{L^{2}\left(\mathbb{R}^{n}\right)}$.

(3) If $u \in \mathscr{S}^{\prime}\left(\mathbb{R}^{n}\right)$, then $h D_{x} T u=\left(\xi+\sqrt{-1} h D_{\xi}\right) T u=T\left(-h D_{y} u\right)$.

(4) We have $u=T^{*} T u$, where $T^{*}$ is defined as

$$
T^{*} v(y)=2^{\frac{n}{2}} \pi h^{-\frac{3 n}{4}} \int e^{-\frac{\sqrt{-1}}{h}(x-y) \xi-\frac{1}{2 h}(x-y)^{2}} v(x, \xi) d x d \xi
$$

which can be interpreted as an oscillatory integral with respect to $\xi$.

The standard FBI transformation is easy to use, but it is not invariant under change of variables. Introduce a general analytic phase function

$$
\varphi(\beta, x), \beta=\left(\beta_{x}, \beta_{\xi}\right) \in \mathbb{R}^{2 n}, x \in \mathbb{R}^{n}
$$


satisfying the same microlocal properties as $\left(\beta_{x}-x\right) \beta_{\xi}-\frac{1}{2 \sqrt{-1}}\left(\beta_{x}-x\right)^{2}$ :

$$
\begin{aligned}
& \varphi=0, \varphi_{x}^{\prime}=\sqrt{-1} \beta_{x}, \quad \text { for } \beta_{x}=x \\
& \operatorname{Im} \varphi \geq C\left|\beta_{x}-x\right|^{2}, \quad \text { for real } \beta, x
\end{aligned}
$$

and define the general FBI-transform as

$$
T_{\varphi, h}(u)(\beta)=\int e^{\frac{\sqrt{-1}}{h}} a(\beta, x, h) \chi(x) u(x) d y,
$$

where $a$ is an elliptic analytic symbol, $\chi$ is a cut-off function.

If we just want to consider the microlocal property near $\left(x_{0}, \xi_{0}\right)$, then we only need to define $\beta$ and $\varphi$ close to $\left(x_{0}, \xi_{0}\right)$ and $\chi=1$ near $x_{0}$.

Say $u$ is of exponentially decay microlocally at $\left(x_{0}, \xi_{0}\right) \in T^{*} \mathbb{R}^{n}$ if

$$
T_{\varphi, h}(u)(\beta)=O\left(e^{-\frac{c}{h}}\right), \text { as } h \rightarrow 0
$$

for some $c>0$ uniformly in a neighborhood of $\left(x_{0}, \xi_{0}\right)$. The definition is independent on the choice of $\chi, a$ and $\varphi$.

Definition D.1.3. The analytic wave front set $\mathrm{WF}_{a}(u)$ of $u$ is the set of all points $\beta=(x, \xi) \in T^{*} \mathbb{R}^{n} \backslash 0$ where $u$ is not of exponentially decay microlocally.

Proposition D.1.4. We have $\mathrm{WF}_{a}(u) \subset T^{*} \mathbb{R}^{n} \backslash 0$. Moreover, Sato's definition of singular spectrum S. S. (u) equal to $\mathrm{WF}_{a}(u) / \mathbb{R}_{+}$.

Example D.1.5. For $\tau \geq 1$, consider the function

$$
u= \begin{cases}e^{-\frac{1}{x^{\tau}}} & \text { for } x>0 \\ 0 & \text { for } x \leq 0\end{cases}
$$

We have $\mathrm{WF}(u)=0, \mathrm{WF}_{a}(u)=\{(0, \xi): \xi \neq 0\}$.

Let us check the case $\tau=1$. Since

$$
\left(\frac{d}{d x}\right)^{n} e^{-\frac{1}{x}}=e^{-\frac{1}{x}} x^{-n-1}\left[(-1)^{n+1} n !+O(x)\right],
$$

and the maximum of $e^{-\frac{1}{x}} x^{-n-1}$ is achieved at $x=\frac{1}{n+1}$. In a neighborhood of the origin,

$$
\left|\left(\frac{d}{d x}\right)^{n} e^{-\frac{1}{x}}\right|=O\left(e^{-n-1}(n+1)^{n+1} n !\right) .
$$

For FBI transform

$$
T_{h} u(0, \xi)=\int_{0}^{\infty} e^{-\frac{1}{x}-\frac{\sqrt{-1}}{h} x \xi-\frac{1}{2 h} x^{2}} d x, \quad \xi= \pm 1 .
$$


By scaling $x=t \sqrt{h}$, we have

$$
T_{h} u(0, \pm 1)=\sqrt{h} \int_{0}^{\infty} e^{-\frac{1}{\sqrt{h}}\left[\frac{1}{t}+\sqrt{-1} t \xi+\frac{t^{2}}{2} \sqrt{h}\right]} d t .
$$

Heuristically, the critical point of the phase is at $t=e^{\mp \frac{\pi}{4} \sqrt{-1}}$ and by method of stationary phase, the leading term of $T_{h} u(0, \pm 1)$ will be $\sqrt{h} e^{\mp \frac{\pi}{4} \sqrt{-1}} e^{-\frac{\sqrt{2}}{\sqrt{h}}}$. It shows that $(0, \xi) \in \mathrm{WF}_{a}(u)$.

Remark D.1.6. Usually, there are two ways to study analytic singularities. One is introduced in this section using FBI transform to study analytic wavefront set, the other is use Sato's hyperfunctions and microfunctions to study the analytic spectrum. Those two ways are in some sense equivalent, for instance the equivalence as showed in Bony [5] in the category of distributions. We can choose a suitable way to deal with different problems. 



\section{Appendix E}

\section{Euler's hypergeometric differential equation}

Every second order linear ordinary differential equation with three regular singular points can be transformed into a standard form, i.e. the Euler's hypergeometric differential equation. Solutions of such equations gives the Gaussian hypergeometric functions, which are special functions represented by the hypergeometric series. The Gaussian hypergeometric functions includes many other special functions as specific or limiting cases. For more information, one can check [14], [19], [76].

\section{E.1 Euler's hypergeometric differential equation}

The Euler's hypergeometric differential equation is an second order ordinary differential equation of form

$$
z(1-z) \frac{d^{2} w(z)}{d z^{2}}+[c-(a+b+1-z)] \frac{d w(z)}{d z}-a b w(z)=0,
$$

which has three regular points: 0,1 and $\infty$.

The Euler's hypergeometric differential equation is a special case of Riemann's differential equation (also known as Papperitz equation), with three regular singular points $p, q, r$ on the Riemann sphere, rather than merely at 0,1 , and $\infty$,

$$
\begin{aligned}
\frac{d^{2} w(z)}{d z^{2}}+ & {\left[\frac{1-\alpha-\alpha^{\prime}}{z-p}+\frac{1-\beta-\beta^{\prime}}{z-q}+\frac{1-\gamma-\gamma^{\prime}}{z-r}\right] \frac{d w}{d z}+\left[\frac{\alpha \alpha^{\prime}(p-q)(p-r)}{z-p}\right.} \\
& \left.+\frac{\beta \beta^{\prime}(q-r)(q-p)}{z-q}+\frac{\gamma \gamma^{\prime}(r-p)(r-q)}{z-r}\right] \frac{w}{(z-p)(z-q)(z-r)}=0 .
\end{aligned}
$$

The pairs of the exponents with respect to the singular points $p ; q ; r$ are $\alpha, \alpha^{\prime} ; \beta, \beta^{\prime} ; \gamma, \gamma^{\prime}$ respectively subject to the condition

$$
\alpha+\alpha^{\prime}+\beta+\beta^{\prime}+\gamma+\gamma^{\prime}=1
$$


The equation is completely determined by the position of the three singular points and the pairs of exponents at each point, i.e., a total of eight complex parameters (not nine, because of Formula (E.2)).

Theorem E.1.1. Any second order ordinary differential equation with three regular singular points can be converted to Euler's hypergeometric differential equation (E.1) by a change of variables.

Proof. See [76], Chapter 15. 


\section{Appendix F}

\section{Hermite functions}

Hermite functions are solutions of Schrödinger equation for one harmonic oscillator in quantum mechanics. We need them to study the analytic singularities in the cases of the elliptic radial point and the mixed type radial point in sections 5.2, 6.1.1 and 6.1.3.

\section{F.1 Hermite functions}

Definition F.1.1. There are two different definitions of Hermite polynomials. One is the Hermite polynomials which has usually been used in probability theory:

$$
H e_{n}(x)=(-1) e^{\frac{x^{2}}{2}} \frac{d^{n}}{d x^{n}} e^{-\frac{x^{2}}{2}},
$$

and the other is the physicists' Hermite polynomials:

$$
H_{n}(x)=(-1) e^{x^{2}} \frac{d^{n}}{d x^{n}} e^{-x^{2}} .
$$

Each one of the Hermite polynomials is a re-scaling of the other.

$$
H_{n}(x)=2^{\frac{n}{2}} H e_{n}(\sqrt{2} x), \quad H e_{n}(x)=2^{-\frac{n}{2}} H_{n}\left(\frac{x}{\sqrt{2}}\right) .
$$

Without loss of generality, we will follow the physicists' definition of Hermite polynomials.

The Hermite polynomials are given by the exponential generating function

$$
e^{2 x t-t^{2}}=\sum_{n=0}^{\infty} H_{n}(x) \frac{t^{n}}{n !} .
$$


Definition F.1.2. The Hermite functions are defined by

$$
\begin{aligned}
\varphi_{n}(x) & =\left(2^{n} n ! \sqrt{\pi}\right)^{-\frac{1}{2}} e^{-\frac{x^{2}}{2}} H_{n}(x) \\
& =(-1)^{n}\left(2^{n} n ! \sqrt{\pi}\right)^{-\frac{1}{2}} e^{-\frac{x^{2}}{2}} \frac{d^{n}}{d x^{n}} e^{-x^{2}} .
\end{aligned}
$$

These functions satisfy

$$
\int_{-\infty}^{\infty} \varphi_{n}(x) \varphi_{m}(x) d x=\delta_{n m}
$$

and form an orthonormal basis of $L^{2}(\mathbb{R})$. Notice that the Hermite functions $\varphi_{n}(x)$ satisfy a Schrödinger equation for one harmonic oscillator in quantum mechanics:

$$
\varphi_{n}^{\prime \prime}(x)+\left(2 n+1-x^{2}\right) \varphi_{n}(x)=0 .
$$

Proposition F.1.3. The Hermite functions are exponential decreasing real analytic functions.

Proposition F.1.4. The Hermite functions $\varphi_{n}(x)$ form an orthonormal basis of $L^{2}(\mathbb{R})$ which diagonalizes the Fourier transform operator.

Proof. We have

$$
e^{-\frac{x^{2}}{2}+2 x t-t^{2}}=\sum_{n=0}^{\infty} e^{-\frac{x^{2}}{2}} H_{n}(x) \frac{t^{n}}{n !} .
$$

The Fourier transformation of the left hand side is given by

$$
\begin{aligned}
F\left(e^{-\frac{x^{2}}{2}+2 x t-t^{2}}\right)(k) & =(2 \pi)^{-\frac{1}{2}} \int_{-\infty}^{\infty} e^{-i x k} e^{-\frac{x^{2}}{2}+2 x t-t^{2}} d x \\
& =e^{-\frac{k^{2}}{2}-2 i k t+t^{2}} \\
& =\sum e^{-\frac{k^{2}}{2}} H_{n}(k) \frac{(-i t)^{n}}{n !}
\end{aligned}
$$

The Fourier transformation of the right hand side is

$$
F\left(\sum_{n=0}^{\infty} e^{-\frac{x^{2}}{2}} H_{n}(x) \frac{t^{n}}{n !}\right)=(-i)^{n} e^{-\frac{k^{2}}{2}} H_{n}(k) .
$$

Then we have

$$
F\left(e^{-\frac{x^{2}}{2}} H_{n}(x)\right)=(-i)^{n} e^{-\frac{k^{2}}{2}} H_{n}(k) .
$$

The Proposition F.1.4 gives an essential explanation of Proposition C.3.7 (b). 


\section{Bibliography}

[1] V. I. Arnold, Mathematical Methods of Classical Mechanics (second edition), Graduate Texts in Math., vol. 60, Springer-Verlag, New York, 1989.

[2] M. F. Atiyah and I. G. MacDonald, Introduction to commutative algebra, Addison-wesley publishing company, 1969.

[3] J. E. Björk, Rings of differential operators, North-Holland Mathematical Library, vol. 21, North-Holland Publishing Co., Amsterdam-NY, 1979.

[4] J. E. Björk, Analytic D-modules and applications, Mathematical and its applications, vol. 247, Kluwer Academic publishers group, Dordrecht, 1993.

[5] J. M. Bony, Équivalence des diverses notions de spectre singulier analytique, Séminaire Goulaouic-Schwartz (1996-1997), Équations aux dérivées partielles et analyse fonctionnette, Exp. No. 3 12, Centre Math., École Polytech., Palaiseau, 1997.

[6] J. M. Bony and P. Schapira, Propagation des singularitiés analytiques pour les solutions des équations aux dérivées partielles, Annales de l'institut Fourier, vol. 26, No. 1 (1976), 81-140.

[7] J. M. Bony and P. Schapira, Problème de Cauchy, existence et prolongement pour les hyperfonctions solutions d'équations hyperboliques non strictes, C. R. Acad. Sci. Paris, vol. 274 (1972), 188-191.

[8] L. Boutet de Monvel and P. Kree, Pseudo-differential operators and Gevrey classes, Ann. Inst. Fourier 27 (1967), 295-323.

[9] L. Carleson and T. W. Gamelin, Complex dynamics, Universitext: Tracts in Mathematics, Sringer-Verlag, New York, 1993.

[10] K. T. Chen, Equivalence and decompostion of vector fields about an elementary critical point, Amer. J. Math., vol. 85, 1963, 693-722.

[11] J. Chung, S,-Y,Chung and D. Kim, A characterization for Fourier hyperfunctions, Publ. RIMS, Kyoto Univ., vol. 30 (1994), 203-208.

[12] J. F. Conn, Normal forms for analytic Poisson structures, Annals of Mathematics, vol. 119, No. 3, 1984, 577-601. 
[13] J. F. Conn, Normal forms for smooth Poisson structures, Annals of Mathematics, vol. 121, No. 3, 1985, 565-593.

[14] A. O. Daalhuis, Chapter 15: Hypergeometric function, in F. W. J. Olver, D. M. Lozier, Daniel, R. F. Boisvert, and C. W. Clark, NIST Handbook of Mathematical Functions, Cambridge University Press, 2010.

[15] J. J. Duitermaat, Fourier intergral operators, Progress in Mathematics, vol. 130, Birkhäuser, Boston, 1996.

[16] Yu. V. Egorov, On canonical transformations of pseudodifferential operators, Uspehi Mat. Nauk 24 (1969), 235-236.

[17] L. Ehrenpreis, Fourier analysis in several complex variables. Wiley-Interscience, 1970.

[18] J. R. S. Filho, Propagation of singularities through radial points, Comm. in PDEs, 6(4), (1981), 399-435.

[19] A. Erdélyi, W. Magnus,F. Oberhettinger, Fritz and F. G. Tricomi, Higher transcendental functions, vol. I, New York, McGraw-Hill Book Company Inc.,1953.

[20] Urs Graf, Introduction to hyperfunctions and their integral transforms, an applied and computational approach, Birkhäuser Basel, 2010.

[21] J. E. Marsden and M. McCracken, The Hopf bifurcation and its applications, Springer-Verlag New York, 1976.

[22] V. Guillemin and D. Schaeffer, On a certain class of Fuchsian partial differential equations. Duke Math. J., vol. 44 (1977), 157-199.

[23] N. Haber, A normal form around a lagrangian submanifold of radial points, arXiv: $1210.1275 \mathrm{v} 1$ [math.AP]

[24] N. Haber and A. Vasy, Propagation of singularities around a lagrangian submanifold of radial points, arXiv: $1110.1419 \mathrm{v} 1$ [math.AP]

[25] R. Harvey, Hyperfunctions and partial differential equations, Proc. Nat. Acad. Sci. USA, vol. 55 (1966), 1042-1046.

[26] A. Hassell, R. Melrose and A. Vasy, Microlocal propagation near radial points and scattering for symbolic potentials of order zero, Analysis and PDE, vol. 1, No. 2 (2008), 127-196.

[27] E. Hille, Ordinary differential equations in the complex domain, John Wiley and Sons, New York, 1976.

[28] L. Hörmander, An introduction to complex analysis in several variables, Van Nostrand, Princetion, 1966. 
[29] L. Hörmander, The analysis of linear partial differential operators I, II, III, IV, Die Grundlehren der mathematischen Wissenschaften, vol. 256, 257, 274, 275, Springer, Berlin, 1983, 1983, 1985, 1985.

[30] R. Hotta, Introduction to D-modules, Lecture Note Series 1, Inst. of Math. Sci., Madras, 1987.

[31] R. Hotta, K. Takeuchi and T. Tanisaki, D-modules, perverse sheaves, and representation theory, Progree in Math., vol 236, Birkhäuser Boston, 2008.

[32] Y. Ilyashenko and S. Yakovenko, Lectures on analytic differential equations, Graduate studies in Mathematics, vol. 86, American Mathematical Society, Providence, Rhode Island, 2007.

[33] Y. Ito, Fourier hyperfunctions of general type, J. Math. Kyoto Univ., 28-2 (1988), 213-265.

[34] A. Kaneko, Introduction to hyperfunctions, Mathematics and Its Applications (Japanese Series), Kluwer Academic Publishers, 1988.

[35] M. Kashiwara, D-modules and Microlocal Calculus, Translations of Mathematical monographs, vol. 217, American Mathematical Society, 2003.

[36] M. Kashiwara, Algebraic study of systems of Partial differential equations, Mémoires de la S. M. F. 63, Société Mathématique de France, Master's Thesis presented to Univ. of Tokyo (1971) (in Japanese), translated by A. D'Agnolo and J.-P. Schneiders, 1995.

[37] M. Kashiwara, Systems of microdifferential equations (Notes taken by Teresa Monteiro Fernandes), Progress in Math., vol. 34, Birkhäuser, 1983.

[38] M. Kashiwara, Introduction to microlocal analysis (Lectures given at the university of Bern in June 1984), L'Enseignement mathématique, Universitéde Genève, 1986

[39] M. Kashiwara and T. Kawai, Micro-hyperbolic pseudo-differential operators I, $J$. Math. Soc. Japan, vol. 27, No. 3 (1975), 359-404.

[40] ([KKK]) M. Kashiwara, T. Kawai and T. Kimura, Foundations of Algebraic Analysis, Kinokuniya 1980 (In Japanese); English edition, Princetion University Press, 1986.

[41] M. Kashiwara and P. Schapira, Micro-hyperbolic systems, Acta Math., 142 (1979), No. 1-2, 1-55.

[42] M. Kashiwara and P. Schapira, Sheaves on manifolds, Springer-Verlag, 1994.

[43] M. Kashiwara and P. Schapira, An introcdutin to microlocal analysis, Univeridade Federal de Pernambuco, Recife-Brasil, 1984.

[44] K. Kataoka and N. Tose, On microhyperbolic mixed problems, J. Math. Soc. Japan, vol. 43, No. 2, 1991, pp 261-304. 
[45] G. Kato, D. C. Struppa, Fundamentals of algebraic microlocal analysis, Monographs and textbooks in pure and applied mathematics, vol. 217, Marcel Dekker, Inc., New York, 1999.

[46] T. Kawai, On the theory of Fourier hyperfunctions and its applications to parital differential equations with constant coefficents, J. Fac. Sci. Univ. Tokyo, vol. 17 (1970), 467-517.

[47] T. Kawai, Construction of local elementary solution for linear partial differential operators I, Proc. Japan Acad., 47 (1971), 19-23.

[48] T. Kawai, Construction of local elementary solutions for linear partial differential operators with real analytic coefficients I, Publ. Res. Inst. Math. Sci., 7 (1971/72), 363-397.

[49] T. Kawai, Construction of local elementary solutions for linear partial differential operators with real analytic coefficients II, Publ. Res. Inst. Math. Sci., 7 (1971/72), 399-426.

[50] K. W. Kim, S.-Y. Chung and D. Kim, Fourier hyperfunctions as the boundary values of smooth solutions of heat equations, Publ. RIMS, Kyoto Univ., vol. 29 (1993), 289-300.

[51] H. Komatsu, On the regularity of hyperfunction solutions of linear ordinary differential equations with real analytic coefficients, J. Fac. Sci. Univ. Tokyo Sect. IA Math., 20 (1973), 107-119.

[52] H. Komatsu, An introduction to the theory of hyperfunctions, Hyperfunctions and Pseudo-Differential Equations, Lecture Notes in Mathematics, Springer, vol. 287 (1973), 3-40.

[53] H. Lewy, An exmaple of a smooth linear partial differential equation without solutions, Annals of Math, vol. 66, 1957, 155-158.

[54] Ellen S. Livingston and David L. Elliott, Linearization of families of vector fields, Journal of Differential Equations, vol. 55, Issue 3, December 1984, Pages 289-299

[55] P. Maisonobe and C. Sabbah, D-modules cohérents et holonomes, Travaus en cours, vol. 45, Hermann, éditeurs des sciences et des arts, Paris, 1993.

[56] A. Martinez, An introduction to seminclassical and microlocal analysis, Universitext, Springer, Berlin, 2002.

[57] A. Martinez, Le 'edge of the wedge' en théorie des hyperfonctions de Sato, Proc. Intern. Conf. on Functional Analysis and Related Topics, 1969, Univ. of Tokyo Press, Tokyo, 1970, 95-106.

[58] V. P. Maslov, Theorie des Perturbations et Methods asymptotiques, Dunod, Paris, 1972 (French translation of the Russian original published in Moscow, 1965). 
[59] M. Morimoto, An introduction to Sato's hyperfunctions, (Translated and revised from the 1976 Japanese original by the author), Translations of Mathematical Monographs, vol. 129, American Mathematical Society, Providence, RI, 1993.

[60] S. Mizohata, Some remarks on the Cauchy problem, J. Math. Tokyo Univ., vol. 1, 1961, 109-127.

[61] F. Pham, Singularities of integrals: Homology, hyperfunctions and microlocal analysis, Universitext, Translation from the French language edition, Springer-Verlag London limited, 2011.

[62] H. Poincaré, Oeuvres, vol. 1, Gauthier-Villars, Paris, 1928.

[63] ([SKK]) M. Sato, T. Kawai and M. Kashiwara, Microfunctions and pseudo-differential equations. In: Hyperfunctions and pseudo-differential equations, Lecture Notes in Math., vol. 287, Springer, Berlin, 1973, 265-529.

[64] M. Sato, Theory of hyperfunctions I and II, J. Fac. Sci. Univ. Tokyo Sect., I 8 (1959), 139-193; ibid. 8 (1960), 387-437.

[65] M. Sato, Regularity of hyperfunctions solutions of partial differential equations, Actes du Congrès International des Mathématiciens (Nice, 1970), vol. 2, 785-794, Gauthier-Villars, Paris, 1971.

[66] P. Schapira, Microdifferential systems in the complex domain, Grundlehren der Mathematischen Wissenschaften 269, Springer-Verlag, 1985.

[67] J.-P. Schneiders, An introduction to D-modules, Bull. Soc. Roy. Sci. Liege, 1994.

[68] J. L. Sedwick and D. L. Elliott, Linearization of analytic vector fields in the transitive case, Journal of Differential Equations, vol. 25, Issue 3, September 1977, $377-390$

[69] Ana Cannas da Silva, Lectures on Symplectic Geometry, Lecture notes in mathematics, vol. 1764, springer-verlag, 2006.

[70] A. G. Smirnov, Fourier transformation of Sato's hyperfunctions, Advances in Math., 196 (2005), pp 310-345.

[71] S. Sternberg, Local contraction and a theorem of Poincaré, American Journal of Mathematics, vol. 79, No. 4, Oct., 1957, 809-824.

[72] S. Sternberg, The structure of local homeomorphisms, III American Journal of Mathematics, vol. 81, No. 3, July, 1959, 578-604.

[73] S. Sternberg, Lectures on Symplectic Geometry (in Mandarin), Lecture notes of Mathematical Science Center of Tsingua University, International Press, 2012.

[74] M. Taylor, Pseudodifferential operators, Princeton Math. Series, vol. 34, Princeton Univ. Press, Princeton, 1981. 
[75] I. Witt, Maximal Sobolev regularity at radial points. In: Symmetries in algebra and number theory, Göttingen, 2009, 149-160.

[76] M. Abramowitz and I. A. Stegun, Handbook of mathematical functions, With Formulas, Graphs, and Mathematical Tables, Applied Mathematics Series No. 55, Dover Publications, New York, 1972. 